\title{
Assessing the potential of Interferometric SAR for monitoring linear transportation infrastructure: case studies from Eastern Ontario
}

\author{
By \\ Peter Oliver
}

A thesis submitted to the Faculty of Science in partial fulfillment of the requirements for the degree of Master of Science

\author{
Department of Earth Sciences \\ Carleton University \\ Ottawa, Ontario
}

August 2017 
The undersigned recommend to the Faculty of Science acceptance of this thesis

\section{Assessing the potential of Interferometric SAR for monitoring linear transportation infrastructure: case studies from Eastern Ontario}

submitted by Peter Oliver in partial fulfillment of the requirements of the degree of Master of Science.

Dr. Claire Samson, Thesis Co-Supervisor Professor

Department of Earth Sciences Carleton University

Dr. Shawn Kenny, Thesis Co-Supervisor Associate Professor Department of Civil and Environmental Engineering Carleton University

Dr. Dariush Motazedian

Chair, Department of Earth Sciences

Carleton University 


\section{Abstract}

Two RADARSAT-2 Spotlight (SLA24 and SLA74), and one Sentinel-1A (IW) SAR datasets are used to assess the potential of InSAR for monitoring linear transportation infrastructure subject to geohazards. A variety of case studies in Cornwall, Ontario are examined. An InSAR processing sequence was established for the RADARSAT-2 datasets; 19 SLA24 and 15 SLA74 images were used to create time-series deformation maps of Cornwall spanning March 2015 to September 2016. The noise floors were \pm 1.5 $\mathrm{cm}$ and $\pm 1.0 \mathrm{~cm}$, respectively. Phase unwrapping errors, atmospheric path delay, and limited SAR data were identified as the largest contributors to noise. The InSAR processing sequence was adapted to the Sentinel-1A dataset, and used to create unwrapped differential interferograms. A discussion of the phase components of these interferograms is presented. Large SAR datasets, small incidence angles, moderate image resolution, and 6-12 day revisit periods are recommended for monitoring linear transportation infrastructure. 


\section{Acknowledgments}

This project would not have been possible without significant help, guidance, and mentorship from others. I would like to thank my supervisors, Dr. Claire Samson and Dr. Shawn Kenny. Dr. Claire Samson has, throughout my tenure as a student, been an unwavering source of knowledge, generous support, and encouragement to me. I thank Dr. Shawn Kenny for inviting me to join his research group, and for his guidance throughout project. This project was funded by the SOAR-Education program of the Canadian Space Agency; for this I thank Stéphane Chalifoux and Steve Iris, who also provided continual encouragement throughout the project.

I would like to thank Desmond Powers for hosting my internship at C-Core at Memorial University, and to Jerry English for his patient instruction and guidance as I learned the complex task of processing SAR data with the GAMMA Software. I thank Joseph Chamberland for his help in establishing the foundation of this project by guiding me through the process of establishing a dataset and study site. I extend my sincere gratitude to Dr. Pierre-Jean Alasset, who allowed me to make use of his expertise during the processing and interpretation of the data. Without his extremely generous and enthusiastic mentorship this project would not have been possible, nor would I have learned so much in the process.

I thank Dr. Peter Fernberg from the City of Cornwall for his insight, discussions, and major contributions to determining suitable case studies. Denis Lalonde from the City of Cornwall provided the LiDar data for Cornwall, and I thank him for that. I thank 
Daniel Cusson from the National Research Council for his discussion of the InSAR monitoring of the Seaway International Bridge.

Throughout this project, fellow students provided valuable support. Gabrielle Marcotte shared her geotechnical expertise with me during the site selection process, and Michael Cunningham's consistent, level headed advice and discussions were irreplaceable. I thank them both for this support. Finally I thank my family, particularly my parents and my girlfriend, who were a continual source of motivation to me over my entire academic career. 


\section{$\underline{\text { Table of Contents }}$}

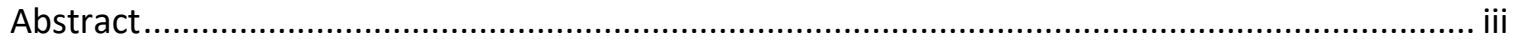

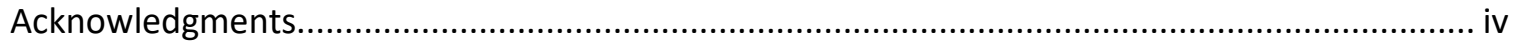

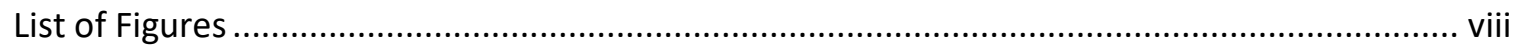

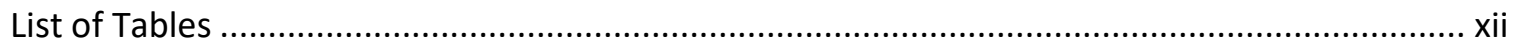

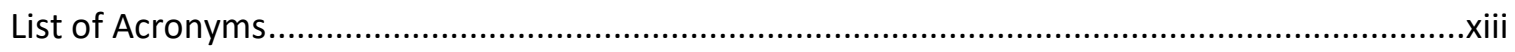

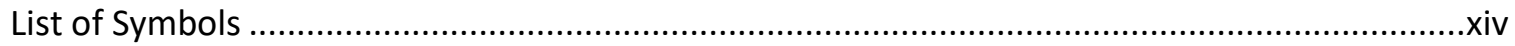

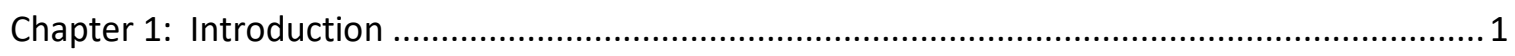

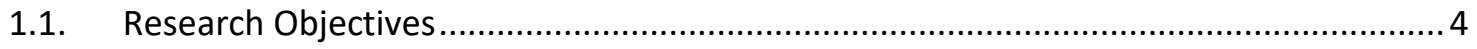

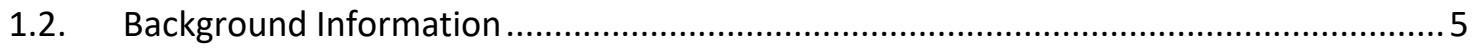

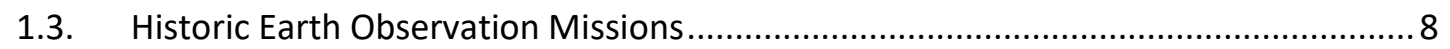

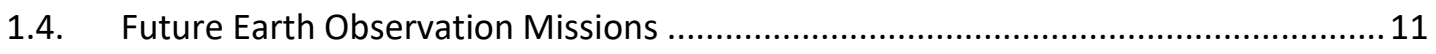

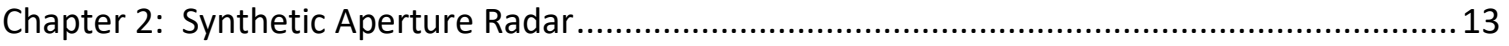

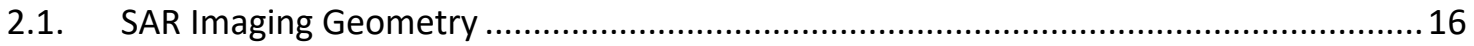

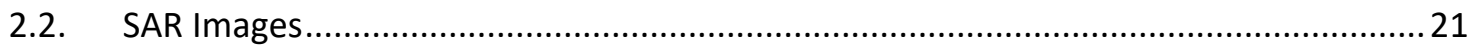

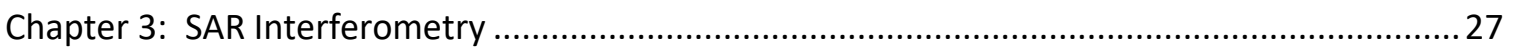

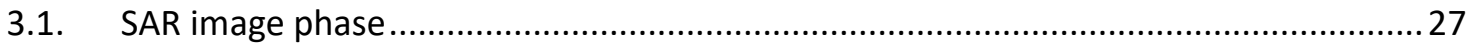

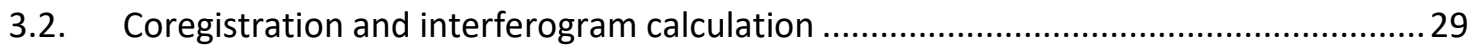

3.3. IPD contributions: flat Earth, topography, and deformation ..................................... 33

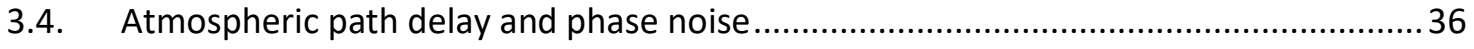

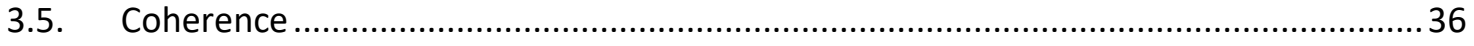

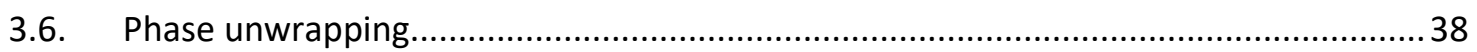

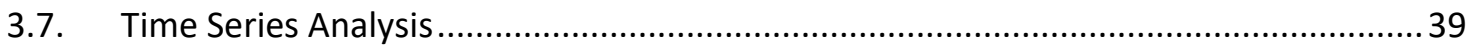

Chapter 4: Geological Setting, Site Selection Criteria, Data Description ................................... 41

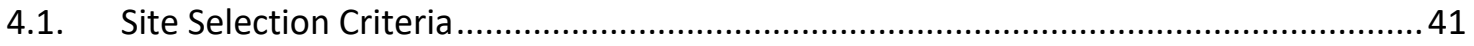

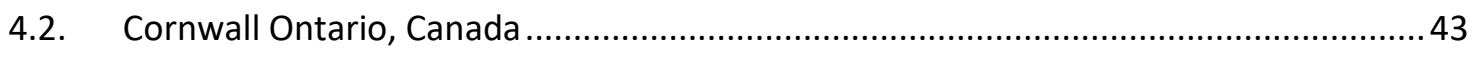

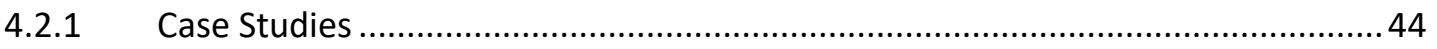

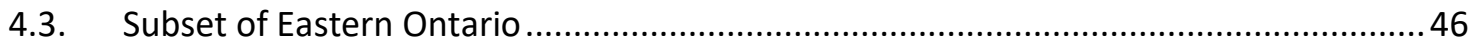

4.4. Geological Setting and Geohazards in Eastern Ontario ..............................................46

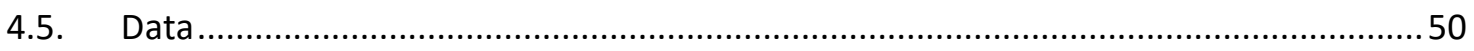

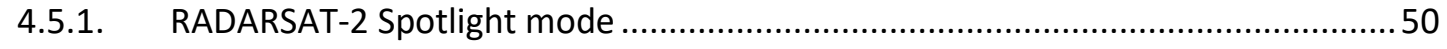




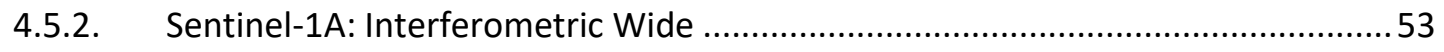

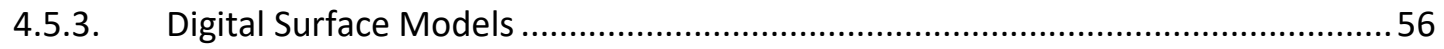

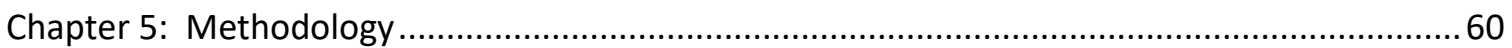

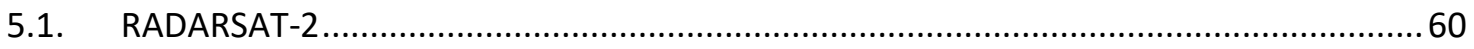

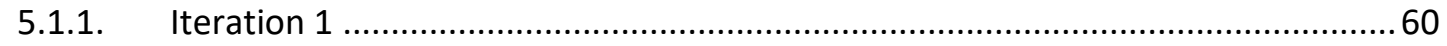

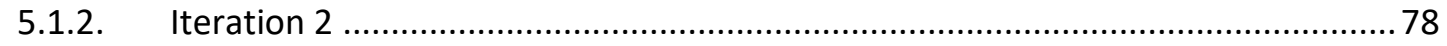

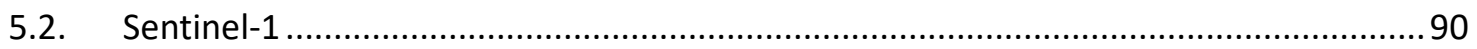

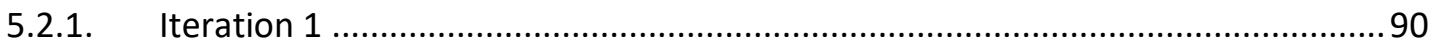

5.2 .2 Iteration 2

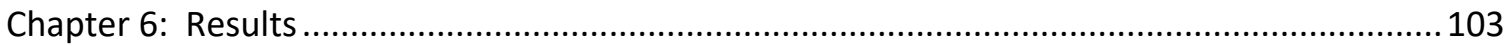

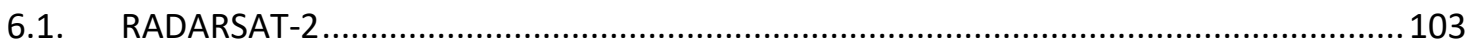

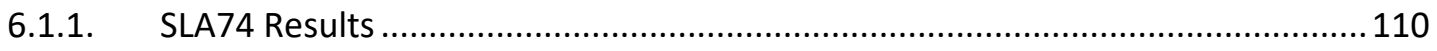

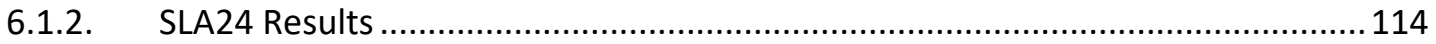

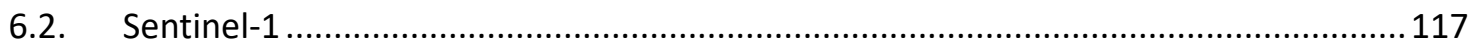

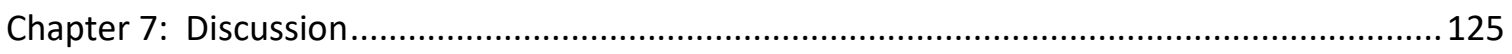

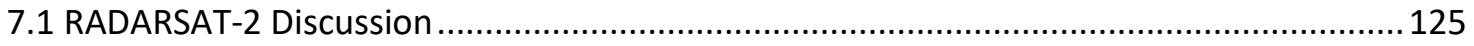

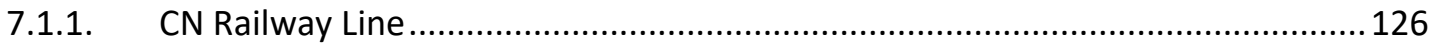

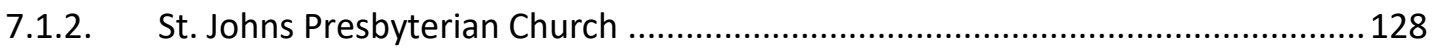

7.1.3. Intersection of Emma Ave and Cumberland St, and Saunders Rd ......................134

7.1.4. R. H. Saunders Generation Station Access Road...............................................136

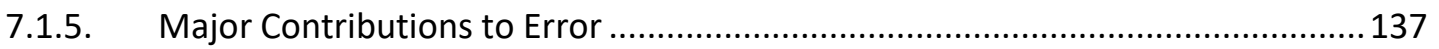

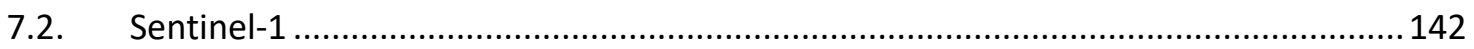

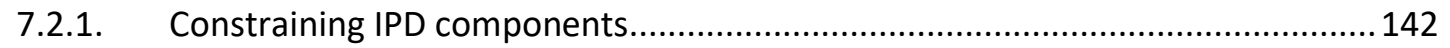

7.2.2. Potential for InSAR Monitoring with S1 Images................................................ 154

Chapter 8: Research Summary and Recommended SAR Specifications...................................156

8.1. SAR Dataset Recommendations for Infrastructure Monitoring ....................................157

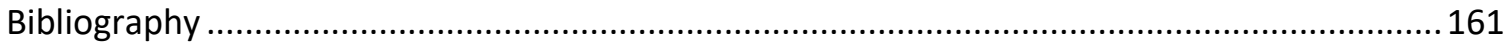

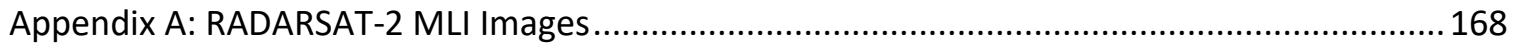

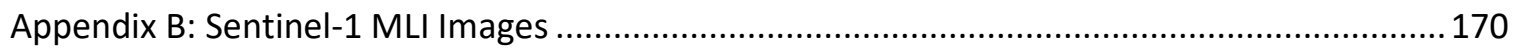

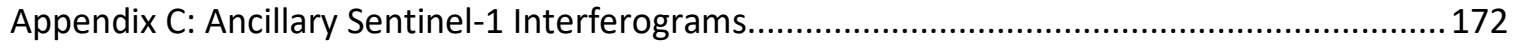

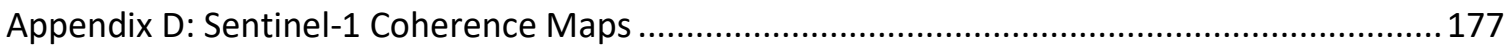

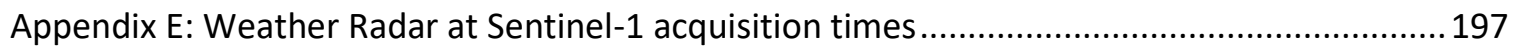




\section{List of Figures}

Figure 1: Differential interferogram and an elastic model of the Landers earthquake... 7

Figure 2: Timeline of most significant past, current, and future EO SAR missions.......... 10

Figure 3: Diagram of a SAR platform and synthetic aperture length. ............................ 15

Figure 4: The imaging geometry of spaceborne SAR platforms................................. 18

Figure 5: Terminology used to describe the imaging geometry of a SAR satellite.......... 19

Figure 6: Ascending and descending orbits for spaceborne SAR platforms................... 20

Figure 7: SAR image showing foreshortened rooftops of several houses....................... 23

Figure 8: Range compression in RDC and correction in GRC................................. 26

Figure 9: Graphical representation of partitioning a SAR image into patches ................. 31

Figure 10: Diagram of the baseline $(B)$ and the perpendicular baseline $\left(B_{\perp}\right)$................ 34

Figure 11: LiDAR Derived 3 m DSM of Cornwall Ontario ............................................ 57

Figure 12: Subset of Natural Resources Canada's 20 m Canadian DSM mosaic .............. 58

Figure 13: Surficial geology of Cornwall, Ontario and a subset of Eastern Ontario......... 59

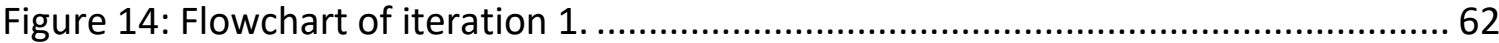

Figure 15: SLC image of the geometrical master and slave SLC image. ......................... 68

Figure 16: The SLA74 study area in RDC and UTM coordinates ................................. 72

Figure 17: Perpendicular baseline vs. temporal baseline ......................................... 74

Figure 18: Interferograms with variable coherence and unwrapping errors .................. 77

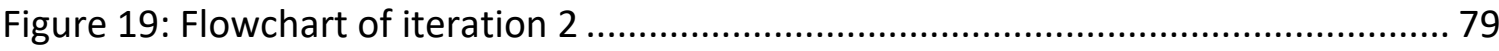

Figure 20: The SLA74 unwrapping coherence mask .................................................. 84

Figure 21: Examples of interferograms with considerable atmospheric effects............. 86

Figure 22: Perpendicular baseline vs. temporal baseline for selected interferograms... 88

Figure 23: Perpendicular baseline vs. temporal baseline for Sentinel-1 data................. 95

Figure 24: An example of an interferogram with a baseline related phase trend.......... 97

Figure 25: Perpendicular baseline vs. temporal baseline for selected interferogram .... 99

Figure 26: An example of baseline refinement. ........................................................... 102 
Figure 27: Average magnitude of the error levels of the reference points................... 106

Figure 28: Average error for each reference point through the time-series. ............... 107

Figure 29: Histogram of pixel values for the SLA74 and SLA24 deformation maps....... 108

Figure 30: RADARSAT-2 study area, reference point locations, case study locations.... 109

Figure 31: Deformation of the north eastern section of the CN Railway line (SLA74) ... 110

Figure 32: Deformation of the Emma and Cumberland intersection (SLA74) ............... 111

Figure 33: Deformation of the St. Johns Presbyterian church (SLA74) ........................ 112

Figure 34: Deformation of the R.H. Saunders Generating Station (SLA74) .................. 113

Figure 35: Deformation of the the Emma and Cumberland intersection (SLA24) ......... 114

Figure 36: Deformation of the St. Johns Presbyterian church (SLA24) ........................ 115

Figure 37: Deformation of the R.H. Saunders Generating Station's (SLA24) ................. 116

Figure 38: Unwrapped, differential interferogram from 2015-09-08 to 2015-09-20 ... 118

Figure 39: Weather radar at 22:50 UTC on $2015-09-08$ and $2015-09-20 \ldots \ldots \ldots \ldots \ldots . . . . . . . . . .119$

Figure 40: Unwrapped, differential interferogram from 2017-01-24 to 2017-02-05 ... 120

Figure 41: Sections of the unwrapped, differential interferogram in Figure 39.......... 121

Figure 42: Unwrapped, differential interferogram from 2016-03-30 to 2016-04-23 ... 122

Figure 43: Unwrapped, differential interferogram from 2016-10-08 to 2016-10-20 ... 123

Figure 44: Unwrapped, differential interferogram from 2015-10-26 to 2016-10-08 ... 124

Figure 45: MLI images of the St. Johns Presbyterian Church ..................................... 132

Figure 46: Layover in the (green) SLA24 and (blue) SLA74 datasets. .......................... 133

Figure 47: Diagram showing the projection of LOS error onto the vertical plane. ....... 139

Figure 48: Interferogram without topographic phase removal created with GAMMA. 145

Figure 49: Interferogram without topographic phase removal created with SNAP ...... 146

Figure 50: Interferogram with topographic phase removal created with GAMMA....... 147

Figure 51: Interferogram with topographic phase removal created with SNAP........... 148

Figure 52: Interferogram (VH) without topographic phase removal from SNAP.......... 150

Figure 53: Interferogram of agricultural area from 2016-10-20 to 2016-11-01. .......... 153

Figure 54: Geocoded MLI Image of the SLA74 study area.......................................... 168

Figure 55: Geocoded MLI Image of the SLA24 study area....................................... 169 
Figure 56: Geocoded MLI image of the Sentinel-1 study area. ................................. 170

Figure 57: Geocoded Sentinel-1 MLI image of Cornwall, Ontario............................... 171

Figure 58: Unwrapped, differential interferogram from 2018-08-15 to 2015-08-27 .... 172

Figure 59: Unwrapped, differential interferogram from 2015-08-27 to 2015-09-08 ... 173

Figure 60: Unwrapped, differential interferogram from2015-11-19 to 2015-12-13 .... 174

Figure 61: Unwrapped, differential interferogram from 2016-10-20 to 2016-11-01 .... 175

Figure 62: Unwrapped, differential interferogram from 2016-11-01 to 2016-11-13 .... 176

Figure 63: Interferometric coherence of 2015-08-15 to 2015-08-27 interferogram..... 177

Figure 64: Interferometric coherence of Cornwall from 2015-08-15 to 2015-08-27.... 178

Figure 65: Interferometric coherence of 2015-08-27 to 2015-09-08 interferogram..... 179

Figure 66: Interferometric coherence of Cornwall from 2015-08-27 to 2015-09-08.... 180

Figure 67: Interferometric coherence of 2015-09-08 to 2015-09-20 interferogram..... 181

Figure 68: Interferometric coherence of Cornwall from 2015-09-08 to 2015-09-20..... 182

Figure 69: Interferometric coherence of 2015-10-26 to 2016-10-08 interferogram..... 183

Figure 70: Interferometric coherence of Cornwall from 2015-10-26 to 2016-10-08.... 184

Figure 71: Interferometric coherence of 2015-11-19 to 2015-12-13 interferogram..... 185

Figure 72: Interferometric coherence of Cornwall from 2015-11-19 to 2015-12-1...... 186

Figure 73: Interferometric coherence of 2016-03-30 to 2016-04-23 interferogram..... 187

Figure 74: Interferometric coherence of Cornwall from 2016-03-30 to 2016-04-23.... 188

Figure 75: Interferometric coherence of 2016-10-20 to 2016-11-01 interferogram..... 189

Figure 76: Interferometric coherence of Cornwall from 2016-10-20 to 2016-11-01.... 190

Figure 77: Interferometric coherence of 2016-10-08 to 2016-10-20 interferogram..... 191

Figure 78: Interferometric coherence of Cornwall from 2016-10-08 to 2016-10-20..... 192

Figure 79: Interferometric coherence of 2016-11-01 to 2016-11-13 interferogram..... 193

Figure 80: Interferometric coherence of Cornwall from 2016-11-01 to 2016-11-13..... 194

Figure 81: Interferometric coherence of 2017-01-24 to 2017-02-05 interferogram..... 195

Figure 82: Interferometric coherence of Cornwall from 2017-01-24 to 2017-02-05..... 196

Figure 83: Weather radar image of the study area at 22:50 UTC on 2015-08-15......... 197

Figure 84: Weather radar image of the study area at 22:50 UTC on 2015-08-27......... 198 
Figure 85: Weather radar image of the study area at 22:50 UTC on 2015-10-26......... 199

Figure 86: Weather radar image of the study area at 22:50 UTC on 2015-11-19......... 200

Figure 87: Weather radar image of the study area at 22:50 UTC on 2015-12-13......... 201

Figure 88: Weather radar image of the study area at 22:50 UTC on 2016-03-30......... 202

Figure 89: Weather radar image of the study area at 22:50 UTC on 2016-04-23......... 203

Figure 90: Weather radar image of the study area at 22:50 UTC on 2016-10-08......... 204

Figure 91: Weather radar image of the study area at 22:50 UTC on 2016-10-20......... 205

Figure 92: Weather radar image of the study area at 22:50 UTC on 2016-11-01......... 206

Figure 93: Weather radar image of the study area at 22:50 UTC on 2016-11-13......... 207

Figure 94: Weather radar image of the study area at 22:50 UTC on 2017-01-24......... 208

Figure 95: Weather radar image of the study area at 22:50 UTC on 2017-02-05......... 209 


\section{List of Tables}

Table 1: RADARSAT-2 acquisition dates, beam modes, and weather conditions. SLA74 images were acquired in an ascending orbit, while SLA24 images were acquired in a descending orbit.

Table 2: Sentinel-1 IW acquisition dates, acquisition times, and weather conditions. All acquisitions are SLC IW images.

Table 3: SLA24 images, image ID's, and the standard deviation of residual offsets (in range and azimuth) of the coregistered images

Table 4: SLA74 images, image ID's, and the standard deviation of residual offsets (in

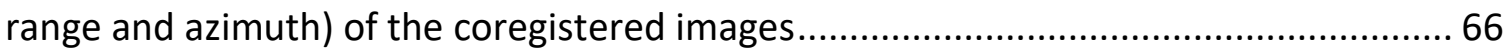

Table 5: Amount of successfully unwrapped interferograms for the SLA74 dataset using coherence masks with various thresholds $(0.45,0.50$, and 0.55$)$ and minimum cost flow triangulation methods (filled triangular mesh or Delaunay triangulation). 83 Table 6: InSAR derived time-series deformations, UTM coordinates, and surficial soil of (assumed to be) stable reference points. 104 


\section{List of Acronyms}

\begin{tabular}{|l|l|}
\hline Acronym & Meaning \\
\hline CSA & Canadian Space Agency \\
\hline CSP & Canadian Space Program \\
\hline DEM & Digital Elevation Model \\
\hline DSM & Digital Surface Model \\
\hline EO & Earth Observation \\
\hline ERS-1\&2 & European Remote Sensing Satellite \\
\hline ESA & European Space Agency \\
\hline EST & Eastern Standard Time \\
\hline GIMP & GNU Image Manipulation Program \\
\hline GRC & Ground Range Coordinates \\
\hline InSAR & Interferometric Synthetic Aperture Radar \\
\hline IPD & Interferometric Phase Difference \\
\hline IPTA & Interferometric Point Target Analysis \\
\hline IW & Interferometric Wide \\
\hline JERS-1 & Japanese Earth Resources Satellite \\
\hline LEO & Low Earth Orbit \\
\hline LiDAR & Light Detection and Ranging \\
\hline LOS & Line Of Sight \\
\hline MCF & Minimum Cost Flow \\
\hline MDA & MacDonald, Dettwiler and Associates \\
\hline MLI & Multilook Intensity \\
\hline MSBAS & Multidimensional Small Baseline Subset Analysis \\
\hline NAD & North American Datum \\
\hline RAR & Real Aperture Radar \\
\hline RCM & RADARSAT Constellation Mission \\
\hline RDC & Range Doppler Coordinates \\
\hline RSLC & Resampled Single Look Complex \\
\hline SAR & Synthetic Aperture Radar \\
\hline SBAS & Small Baseline Subset (Analysis) \\
\hline SLAR & Side Looking Airborne Radar \\
\hline SLC & Single Look Complex \\
\hline SNAP & Sentinel Application Platform \\
\hline SRTM & Shuttle Radar Topography Mission \\
\hline TOPSAR & Terrain Observation and Progressive Scans SAR \\
\hline UTC & Coordinated Universal Time \\
\hline UTM & Universal Transverse Mercator \\
\hline & \\
\hline
\end{tabular}




\section{List of Symbols}

\begin{tabular}{|l|l|}
\hline Symbol & Meaning \\
\hline$A$ & real-valued amplitude (transmitted signal) \\
\hline$a$ & Azimuth (direction) \\
\hline$B$ & Baseline \\
\hline$B_{\perp}$ & Perpendicular Baseline \\
\hline$C$ & Speed of light \\
\hline$C$ & Cost function \\
\hline$F$ & Transmission frequency \\
\hline$f(x, y)$ & SAR Image \\
\hline$H$ & Flight altitude \\
\hline$I_{f g}(x, y)$ & Interferogram \\
\hline$R$ & Sensor-Target distance (Slant-Range) \\
\hline$r$ & Range (Direction) \\
\hline$x(t)$ & Transmitted SAR signal \\
\hline$y(t)$ & Received SAR signal \\
\hline$Z$ & Topographic height \\
\hline$\alpha$ & grazing angle \\
\hline$B$ & Depression angle \\
\hline$\gamma$ & Interferometric coherence \\
\hline$\varepsilon$ & Phase noise and atmospheric path delay \\
\hline$\lambda$ & Wavelength \\
\hline$\rho$ & Complex reflectivity of ground surface \\
\hline$\tau$ & pulse duration of the transmitted signal \\
\hline$\psi_{f g}(x, y)$ & Unwrapped Phase \\
\hline$\varphi$ & Phase \\
\hline$\varphi_{f g}(x, y)$ & Interferometric phase difference image \\
\hline$\varphi_{R x}$ & Phase of received signal \\
\hline$\varphi_{T x}$ & Phase of transmitted signal \\
\hline$\chi$ & Off-nadir angle \\
\hline$\theta$ & incidence angle \\
\hline & \\
\hline
\end{tabular}




\section{Chapter 1: Introduction}

Interferometric Synthetic Aperture Radar (InSAR) is a geodetic technique that exploits differences in the received phase of two or more SAR images to measure topography or deformation of the Earth's surface (Hanssen 2001). A SAR image is formed by the transmission and reception of coherent pulses of electromagnetic radiation, and therefore the difference in received phase of corresponding pixels in two SAR images allows for the measurement of the travel path difference. After removal of various error and noise components, this phase difference represents the cumulative deformation of the Earth's surface that occurred in the time interval between the two images.

Deformation of the Earth's surface often reflects an underlying physical process; for example the deformation of glaciers, permafrost, and wetland regions can be indicative of climate change processes, and seismic deformation can represent pre- and postearthquake strain change. Often, these deformations additionally represent a potential geohazard. For instance mass movements involve surficial deformation of the Earth and present a geohazard across Canada (Bobrowsky et al. 2012). Eastern Ontario and portions of southern Quebec (i.e. the Saint Lawrence Lowlands) have a particularly high lateral spreading hazard resulting from the deposition of sensitive marine clays during the invasion of the Champlain Sea (Quinn 2009). Numerous large lateral spreads have occurred in these Champlain Sea sediments: the lateral spreads in 1908 at Notre-damede-la-Salette, 1971 at Saint-Jean-Vianney, and in 1971 and 1993 in Lemieux, have all 
resulted in either human casualties, or property and infrastructure damage. Champlain Sea sediments frequently cause geotechnical issues such as rapid and differential settlement, notably evident in Orleans, Ottawa (Hewitt 2005).

Assessing the impact of geohazards on linear transportation infrastructure is difficult due to the nature of these systems; they can extend for thousands of kilometres and may cover a wide range of geological environments. Due to this difficulty, most asset integrity management plans are established from age-based and expert knowledge criteria, and not quantitative criteria such as observed deformation (Félio 2012, Vanier et al. 2004). Currently, deformation monitoring of linear transportation infrastructure (highways, railways, bridges etc.) and urban structures (houses, buildings etc.) is performed with traditional land based techniques such as leveling and GPS measurements. These techniques produce accurate, reliable measurements of structural deformations but can only provide such information for a sparse network of points over limited spatial extend. Furthermore, these techniques are labour intensive and require field work. The expense and logistical requirements of implementing these techniques over a large infrastructure network results in their use only on an at-need basis. They are therefore not included in most asset integrity management plans (Félio 2012). If deformation of linear infrastructure could be monitored at a lower cost, over a large spatial extend, and on a regular basis, then these observations could be used to improve current asset integrity management plans. 
This thesis is organized as follows. In Chapter 1 the research goals of this project and background information is presented. Chapter 2 is a brief overview of the SAR concept, with particular focus on how the acquisition geometry influences SAR image interpretation. In Chapter 3, the basis for deformation measurement using InSAR techniques is presented, and includes a discussion of the fundamental InSAR processing steps and the interferometric phase difference model. In Chapter 4, the study location and data used in this project are described. In Chapter 5 the InSAR processing sequence applied in this project is explained, and the corresponding results are presented in Chapter 6. Chapter 7 holds a discussion of these results with a focus on interpreting these results with respect to the SAR image characteristics presented in Chapter 2. In Chapter 8, conclusions on the analysis outcomes and recommendations for SAR data to be used in future projects are made. 


\subsection{Research Objectives}

There are three main objectives to this research project:

1. Use InSAR to monitor linear transportation infrastructure that may be subject to geohazards, i.e. identify, quantify, and monitor ground deformation and assess impacts on infrastructure.

2. Test the current limitations of InSAR for the monitoring of linear transportation infrastructure with respect to the characteristics of SAR data and state of the practice processing techniques.

3. Provide recommendations for SAR data specifications and processing techniques for future linear transportation monitoring projects. These recommendations are presented with particular focus on how the upcoming RADARSAT Constellation Mission (RCM) can be used for this purpose.

Funding for this project has been provided by the Canadian Space Agency (CSA) through the SOAR-Education program. The overarching objectives of the CSA for sponsoring academic research are to:

1. Explore the feasibility of using RADARSAT-2 SAR data for new applications.

2. Build capacity within Canada's remote sensing industry, i.e. to develop a critical mass highly qualified personnel.

The RADARSAT-2 data used in this project was also provided by the CSA through the SOAR-Education program. 


\subsection{Background Information}

The first exploitation of satellite-based InSAR dates back to the 1980s when the California Institute of Technology's Jet Propulsion Laboratory published a proof of concept using SEASAT data (Gabriel et al. 1989). The potential of the InSAR technique for land monitoring was not fully realized until the 1990's when the European, Japanese, and Canadian Space Agencies launched the ERS-1, JERS-1 and RADARSAT-1 satellites, respectively (Ferretti et al. 2007a). These platforms had stricter orbital control, better defined orbits, and provided larger datasets than SEASAT.

The first major demonstration of the value of the InSAR technique to detect surface deformation was a study of the 1992 Landers earthquake (Massonnet et al. 1993). On June 18, 1992, a Richter magnitude 7.3 earthquake occurred northwest of Los Angeles, near the city of Landers, after which the event has been named. The desert region near Landers had previously been heavily instrumented with geodetic and seismological sensors, allowing for a good understanding of the earthquake's rupture mechanisms to be established: this earthquake was a right-lateral strike slip event involving the rupture of the Johnson Valley, Landers, Homestead Valley, Homestead/Emerson, Emerson Valley and Camp Rock faults (Massonnet et al. 1993). The earthquake resulted in a surface rupture, which extended for $70 \mathrm{~km}$ with a maximum horizontal displacement of $5.5 \mathrm{~m}$ and maximum vertical displacement of $1.8 \mathrm{~m}$ (National Geophysical Data Center 1992). Based on these fault rupture parameters, a relatively accurate elastic model of this event has been established (Massonnet et al. 1993). 
At the time of the earthquake, the European Space Agency's ERS-1 satellite had been stabilized in its 35-day repeat pass orbit for several months, thus high quality SAR imagery was available both pre- and post- earthquake. Massonet et al. 1993 interfered two ERS-1 SAR images, acquired on 24 April 1992 and 7 August 1992, to produce a now famous interferogram that captures the co-seismic deformation of the Landers earthquake. Massonet et al. 1994 produced a similar interferogram using two ERS-1 acquisitions taken on 24 April 1992 and 18 June 1993 (i.e. 14 months separation) and demonstrated that even images taken with such a significant temporal separation could still be useful.

The study of the Landers earthquake had far reaching implications for the potential application of InSAR techniques to study geophysical and geotechnical processes. Firstly, this study demonstrated a remarkable contrast in scales: the ERS-1 satellite orbits at roughly $800 \mathrm{~km}$ altitude, the width of the interferogram in Figure 1 is about $100 \mathrm{~km}$, the magnitude of maximum displacement is on the order of centimetres, and the accuracy of displacements measured is on the order of millimetres (Ferretti et al. 2007a). Secondly, demonstrating that the interference of images acquired 14 months apart can still provide useful observations quelled much of the pessimism related to InSAR; previously it had been hypothesized that mere days between acquisitions would result in too much surface alteration for the technique to be viable. Finally, the resemblance between the elastic model of the Landers earthquake and the fringes present in the interferograms gave credibility to the role of InSAR's for monitoring surface deformation (Massonnet et al. 1993). 


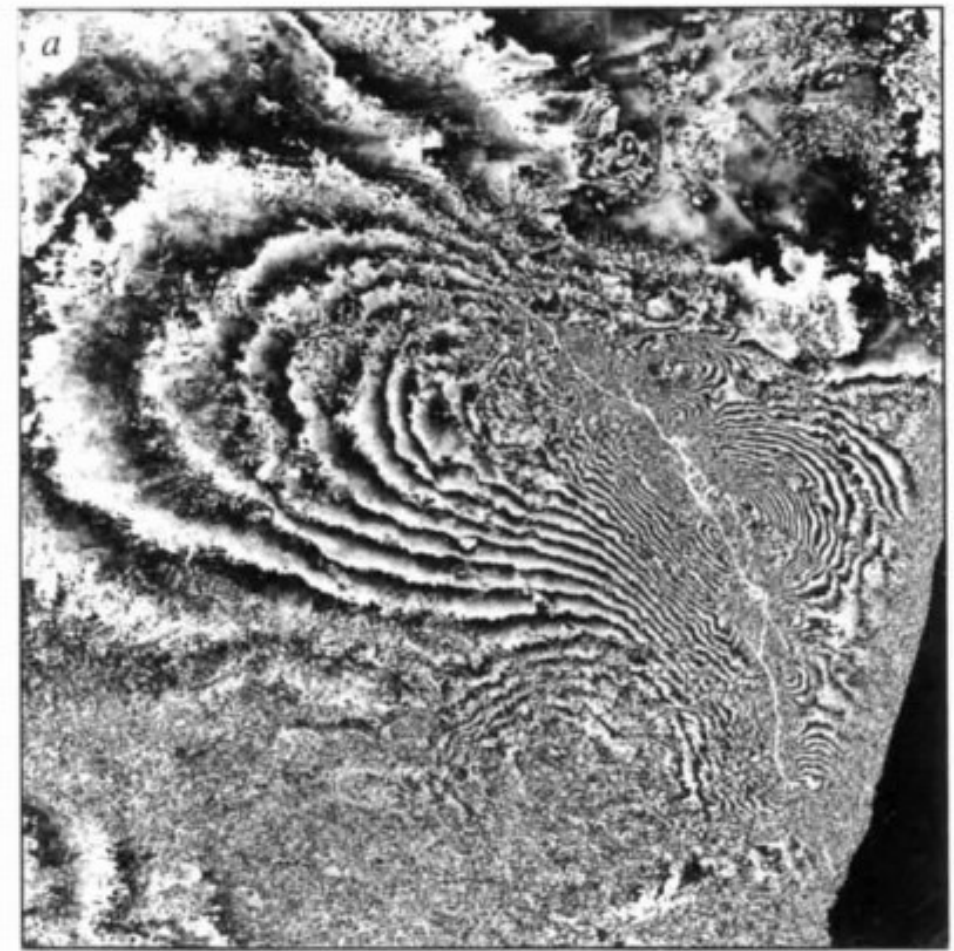

$25 \mathrm{~km}$

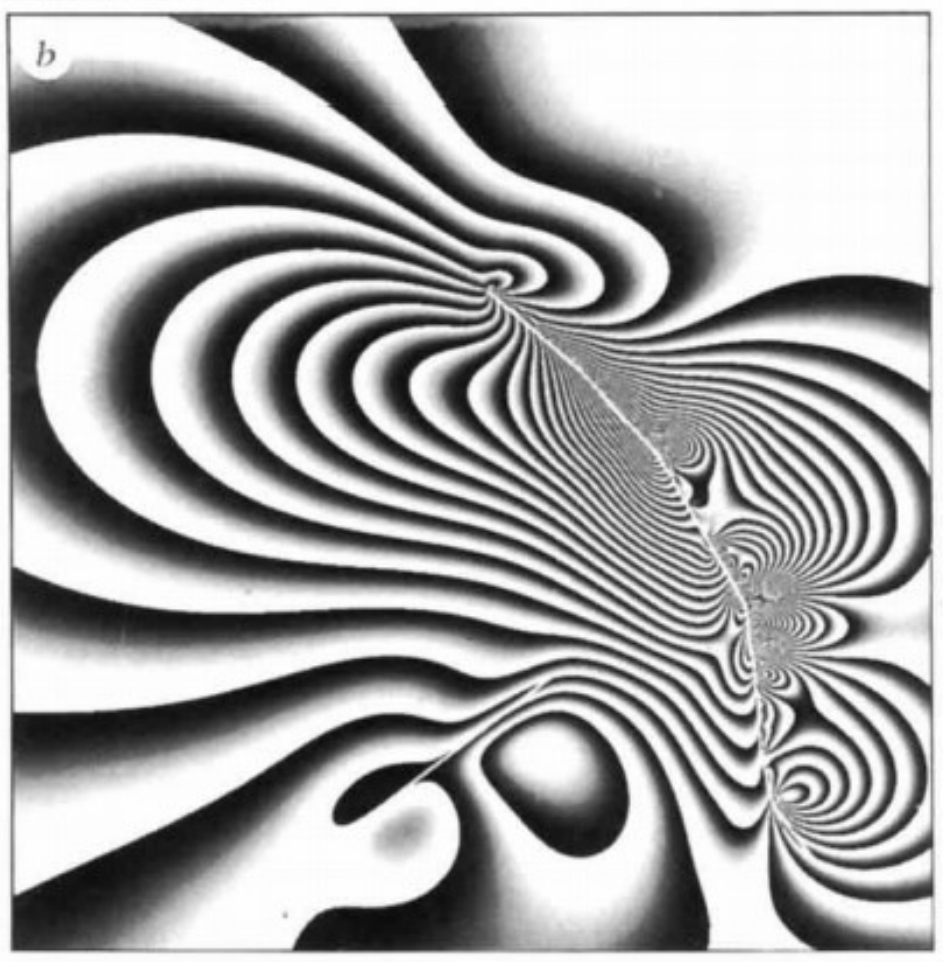

Figure 1: Comparison of a differential interferogram (top) and an elastic half space dislocation model (bottom) of the Landers earthquake. Source: Figure 3 of Massonnet et al. 1993. 


\subsubsection{Historic Earth Observation Missions}

Numerous agencies have supported past, current, and future SAR Earth observation (EO) missions. The SAR satellites used in these missions share similar principles of operation, orbits, and acquisition geometries, but differ in the frequency band: $L$ ( $\lambda=$ $23.6 \mathrm{~cm}), C(\lambda=5.6 \mathrm{~cm})$, and $X(\lambda=3.1 \mathrm{~cm})$ band are commonly used. Figure 2 (UNAVCO 2015) is a timeline of the most significant past, current, and future SAR missions.

The European and Canadian Space Agencies have both been responsible for multiple, internationally significant, C-band SAR missions. The European Space Agency (ESA) launched ERS-1 on July 171991 and its nearly identical successor, ERS-2, on April 211995 (ESA 2017a). The launch of ERS-2 was timed so that it shared the same orbital plane as ERS-1, and this allowed for a tandem mission: for twelve months in 1995-1996, ERS-2 passed over the same point on the ground one day after ERS-1, with both satellites orbiting with a 35 day repeat cycle (ESA 2017b). On March 12002 ESA launched ENVISAT, which was the world's largest civilian EO satellite. In addition to a SAR sensor, ENVISAT carried eight instruments used to monitor land, water, ice, and atmosphere, on a 35 day repeat cycle similar to ERS-1\&2. On May 92012 the ENVISAT mission formally ended.

EO has continually been a key priority for the Canadian Space Program (CSP). Canada's first commercial EO mission was RADARSAT-1, launched November 41995 (CSA 2014). RADARSAT-1 had a 24 day repeat period, offered a variety of beam modes, and was able to rapidly deliver SAR data to users. On March 29 2013, twelve years after 
its expected lifetime, RADARSAT-1 was declared non-operational. The current generation of EO in Canada is RADARSAT-2, launched December 14 2007. RADARSAT-2 is currently operational, 2 years after its expected lifetime (CSA 2015). 


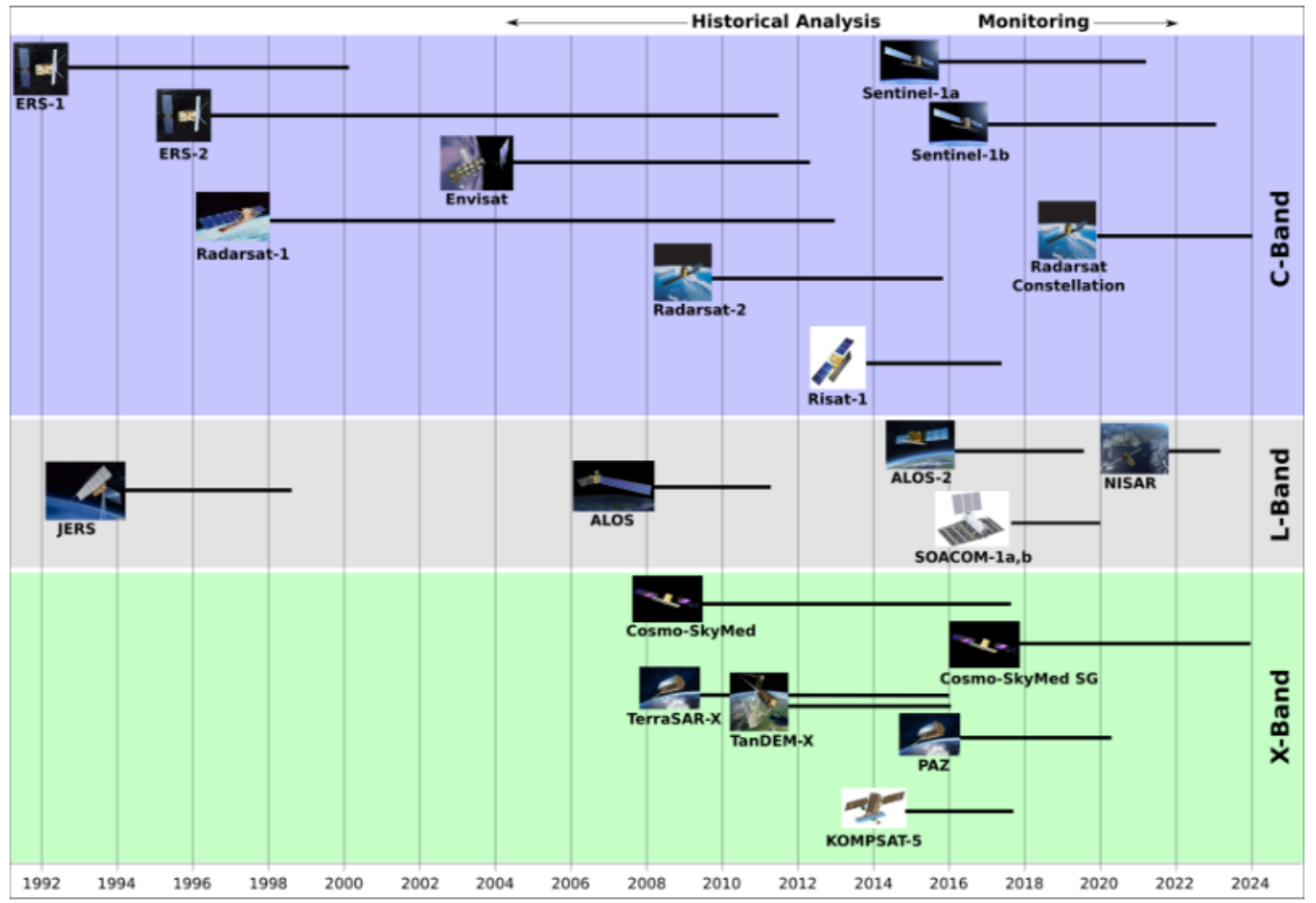

Figure 2: Timeline of most significant past, current, and future EO SAR missions. Source: UNAVCO 2015. 


\subsubsection{Future Earth Observation Missions}

With the exception of the relatively brief ERS 1-2 tandem mission, historical SAR missions have all consisted of a single, often heavily instrumented, platform (UNAVCO 2015). These systems have varied technical specifications and capabilities, but are subject to the same major limitation: SAR interferometry requires multiple SAR images taken from the same position relative to the Earth. Therefore, the minimum time difference between images that can be used for interferometry is equal to the repeat period of the platform under consideration: 35 days for ERS 1\&2, and 24 days for RADARSAT-1\&2. The time period between useable acquisitions is often greater than this since atmospheric effects often make some images unsuitable for interferometry, and higher priority acquisitions (e.g. military applications) often result in the deferral of lower priority acquisitions. The limitations of the single platform, long repeat period SAR platforms have become evident for numerous applications.

Current and near-future SAR missions represent a paradigm shift: instead of a single, large, heavily instrumented platform responsible for performing all capabilities, a constellation approach is implemented. In this approach, multiple smaller systems will be used in conjunction to distribute the same capabilities as the traditional monolithic systems. The main advantages of the constellation approach are: greatly increased observation frequency, better data continuity in heavily solicited regions, greater system resilience to instrumental failure, and lower maintenance and deployment costs due to the smaller size and operational simplicity of the satellites. 
The Sentinel-1 satellite constellation (ESA 2017c) is ESA's successor to ENVISAT, and the first C-band SAR constellation mission. This constellation consists of two satellites: Sentinel-1A, launched on April 3 2014, and Sentinel-1B, launched on April 25 2016. The Sentinel-1 constellation mission operates in a pre-programmed acquisition plan preventing acquisition conflicts and allowing for large, consistent datasets suitable for interferometry to be collected (ESA 2017c).

The next evolution of the RADARSAT Program will be the RADARSAT Constellation Mission (RCM), which is currently in development (CSA 2017). The RCM will consist of three identical satellites each equipped with a C-band SAR sensor. This three-module constellation will allow for a repeat period of 1 day over $90 \%$ of the world's surface. $\mathrm{RCM}$ is intended to fulfill three core objectives: to perform maritime surveillance (monitor the oceans for ice, surface wind, oil pollution, and ship detection), disaster management (to be incorporated into mitigation plans, early warning systems, as well as aid response and recovery efforts), and ecosystem monitoring (forestry and agricultural land use planning, and wetland and coastal change monitoring) (CSA 2017). The RADARSAT Constellation Mission is scheduled to launch in 2018 , and the expected lifetime of each satellite is 7 years. 


\section{Chapter 2: Synthetic Aperture Radar}

Broadly speaking, spaceborne imaging of the Earth's surface is performed with two main types of sensors. Optical imaging systems are those that make use of a lens to focus visible light incident on an aperture, whereas radar imaging systems are based on the echo principle: an antenna emits a coherent beam of microwave radiation, which is absorbed, reflected, and backscattered by the Earth's surface. The backscattered portion of the signal is received by the antenna and processed to form an image. The theory presented in this chapter is based primarily on the content of: Ferretti et al. 2007a, Hanssen 2001, NRCAN 2015, and SkyRadar 2017.

In a radar imaging system that uses a single physical antenna (i.e. in the case of a realaperture radar system), the geometric resolution, $R_{a}$, is proportional to the altitude of the sensor, $H$, the angle the transmitted beam makes with the vertical plane, $\theta$, the wavelength of the transmitted beam, $\lambda$, and the length of the antenna, $d$ :

$$
R_{a}=\frac{H}{\cos (\theta)} \frac{\lambda}{d}
$$

Because of this relationship, in order to achieve suitably high azimuth resolution from the altitude of a spaceborne system (normally between $H=500-800 \mathrm{~km}$ ), operating at C-band $(\lambda=5.6) \mathrm{cm}$, the antenna would have to be extremely large. SAR platforms address this by using the movement of the sensor to synthesise an antenna much larger than can be physically built. 
A physical antenna can be replaced by an array of numerous partial antennas, where the full backscattered signal can be recovered by linear superposition of the signals from each individual partial antenna, provided the backscattered signal was simultaneously received by all partial antennas in the array. In SAR, the motion of a single small antenna simulates an array of partial antennas. Instead of having an array of partial antennas which each receive the backscattered signal at the same time, one small antenna moves forward in space, occupying each position in the (simulated) array at different times (Figure 3) (H\&P Advanced Tech 2017). The movement of one small antenna is equivalent to an array of partial antennas, with the exception that in a physical antenna array the signal is received simultaneously whereas in a simulated array the signals are received at different times. Since the transmitted and received signals are coherent a simulated array can be calculated, creating a so called "synthetic aperture". The SAR sensor receives the signal from a particular ground target for the entire period of time during which it is illuminated by the half-power beam. The length of the synthetic aperture is then the distance travelled by the sensor for the entire period of time during which the target is illuminated. 


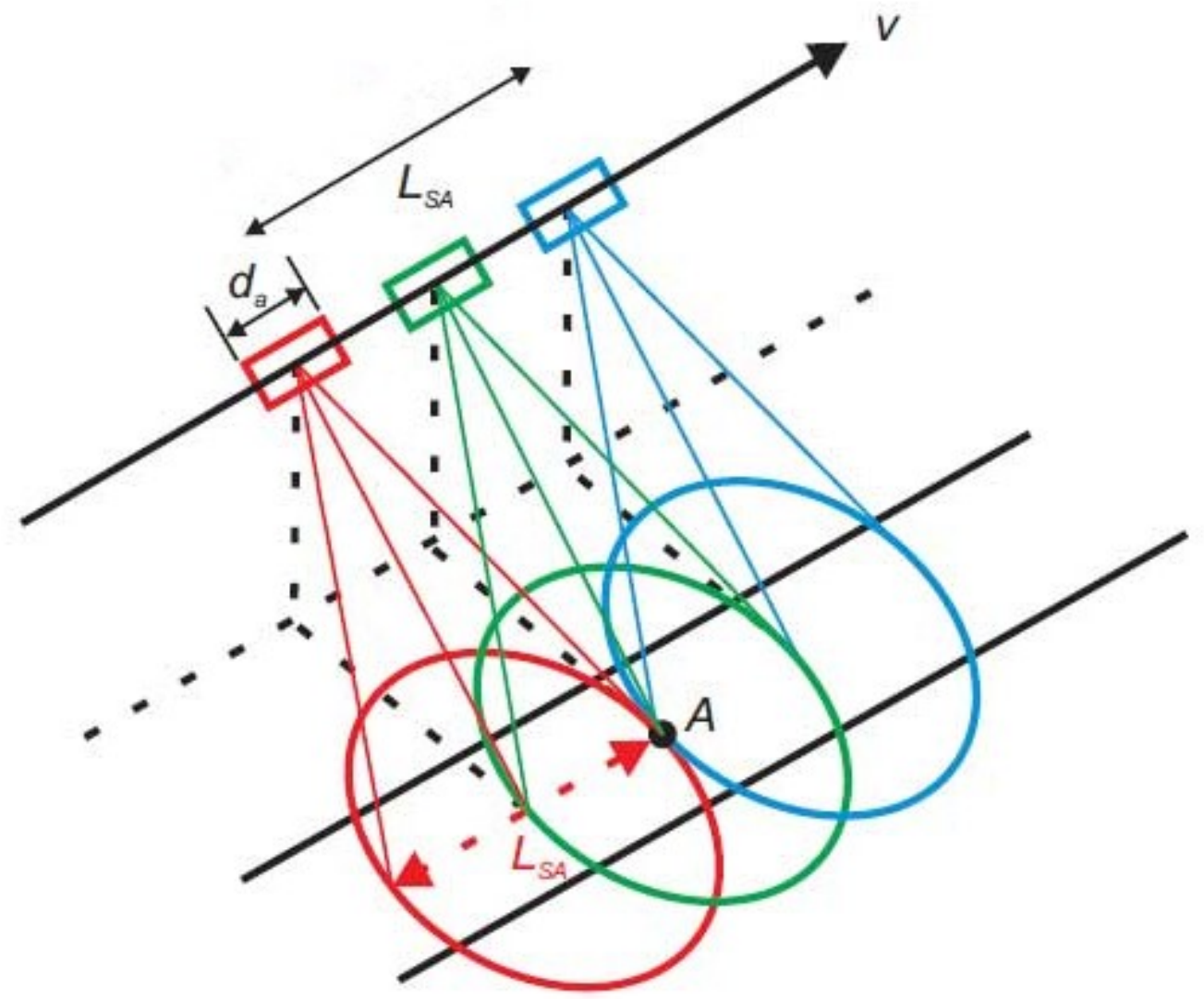

Figure 3: Diagram showing the illumination of a target, A, by a SAR platform with a physical antenna of length, $d_{a}$ moving at velocity, $v$. The length of the synthetic antenna, $L_{s a}$, is the distance travelled by the platform while the target is illuminated. Source: modified from H\&P Advanced Tech 2017. 


\subsection{SAR Imaging Geometry}

The imaging geometry of spaceborne SAR platforms is shown in Figure 4 (SkyRadar 2017). These systems operate in a side-looking radar geometry, i.e. the antenna is mounted perpendicular to the flight direction and can be either left- or rightlooking. The point on the ground directly beneath the aircraft is the nadir point. The direction parallel to the flight direction is the azimuth direction, the direction perpendicular to the flight path is the range direction, and the direction in which the antenna points is the slant-range direction (Casu 2009).

The specific imaging geometry of a particular SAR satellite is described in terms of the angles between these directions, the terminology of which is shown in Figure 5. The angle between the slant-range direction and the horizontal plane, $\beta$, is called the depression angle. Its complement, the off-nadir angle, $\chi$, is made between the sensornadir and slant-range directions. The grazing angle, $\alpha$, is between the horizontal plane tangent to the Earth's surface, and the slant-range direction. The incidence angle, $\theta$, is the angle between the vertical (with respect to the center of the earth) and the slantrange direction. Due to local topography, an additional term, the local-incidence angle, is defined as the angle between the slant-range direction and the normal to the local topography. Because of the curvature and topography of the Earth, the depression angle is not necessarily equal to the grazing angle, nor is the off-nadir angle necessarily equal to the incidence angle. 
SAR satellites operate in a near-polar orbit, as shown in Figure 6 (SkyGeo 2017). The satellite orbit passes close to the North and South poles while the Earth rotates beneath, allowing the whole planet to be imaged by a single platform. The SAR satellite orbit is also sun-synchronous, such that any particular point on the Earth is imaged at the same local time, assuring constant illumination characteristics from one pass to the next. Since the Earth is rotating, each subsequent orbit images a strip to the west of the one previously acquired. When the satellite is orbiting from the South to the North pole it is in an ascending orbit, and conversely, when it is orbiting from the North to South pole it is in a descending orbit. 


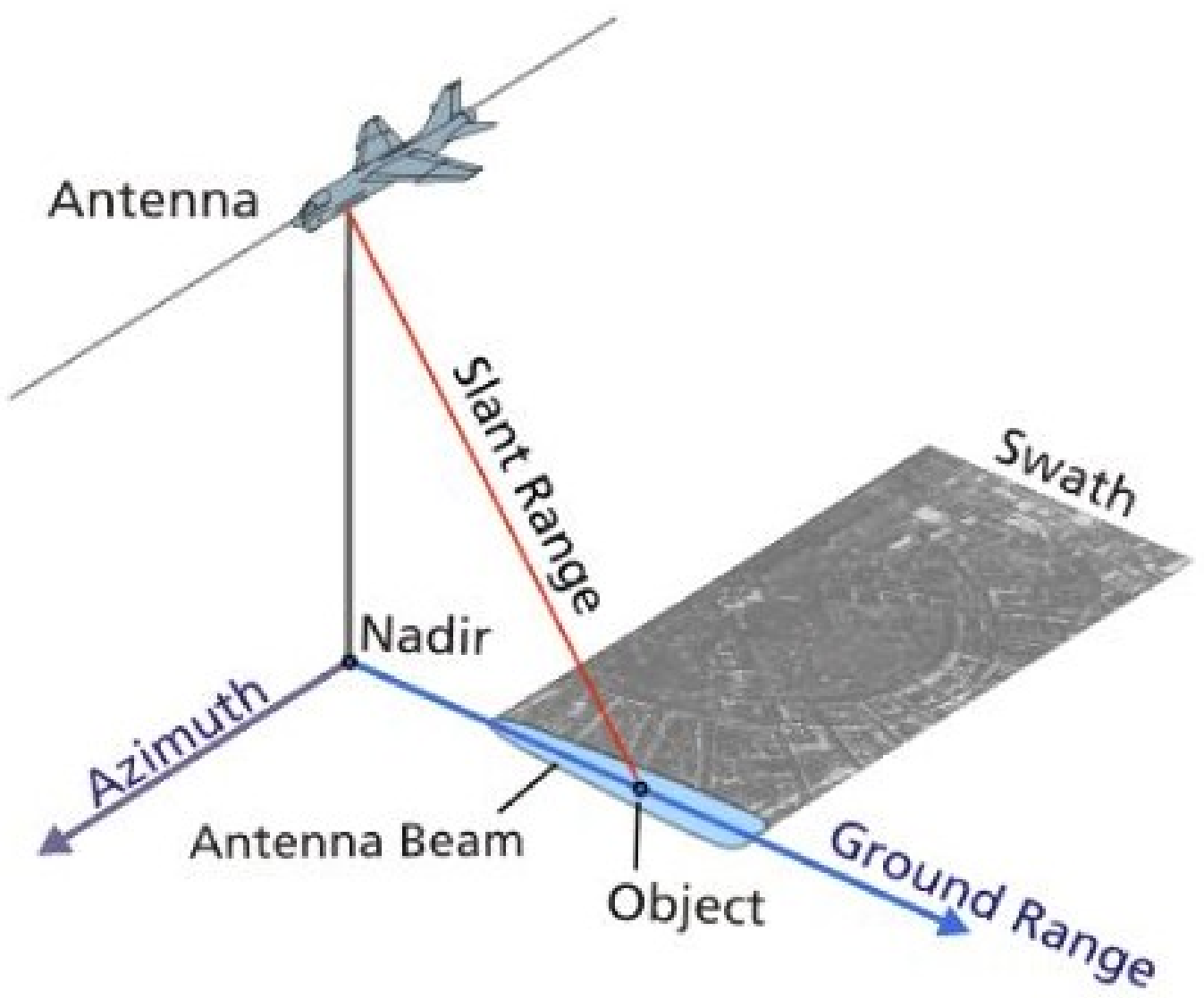

Figure 4: The imaging geometry of spaceborne SAR platforms. Source: SkyRadar 2017. 

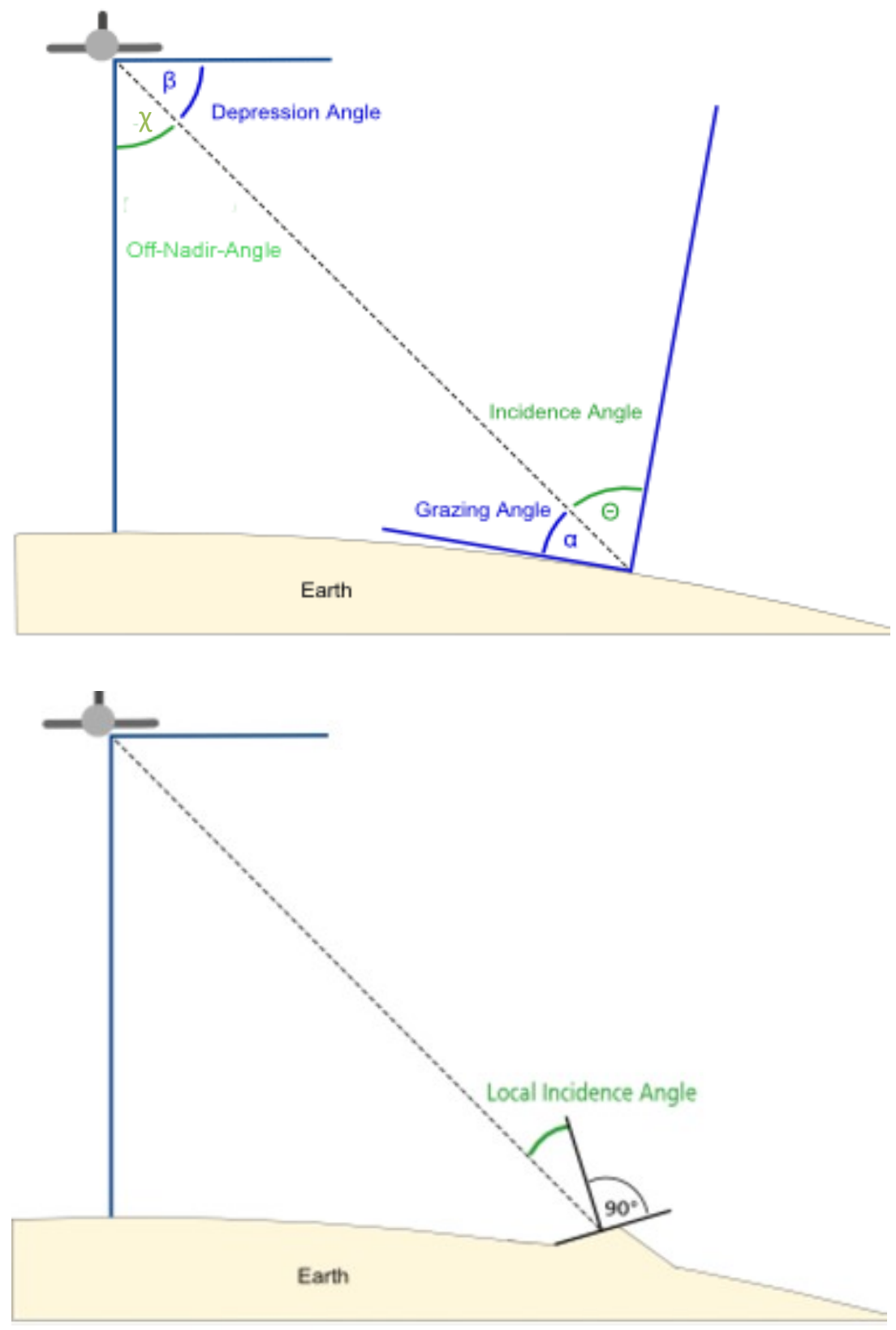

Figure 5: Terminology used to describe the specific imaging geometry of a particular SAR satellite. Source: SkyRadar 2017. 


\section{Descending}

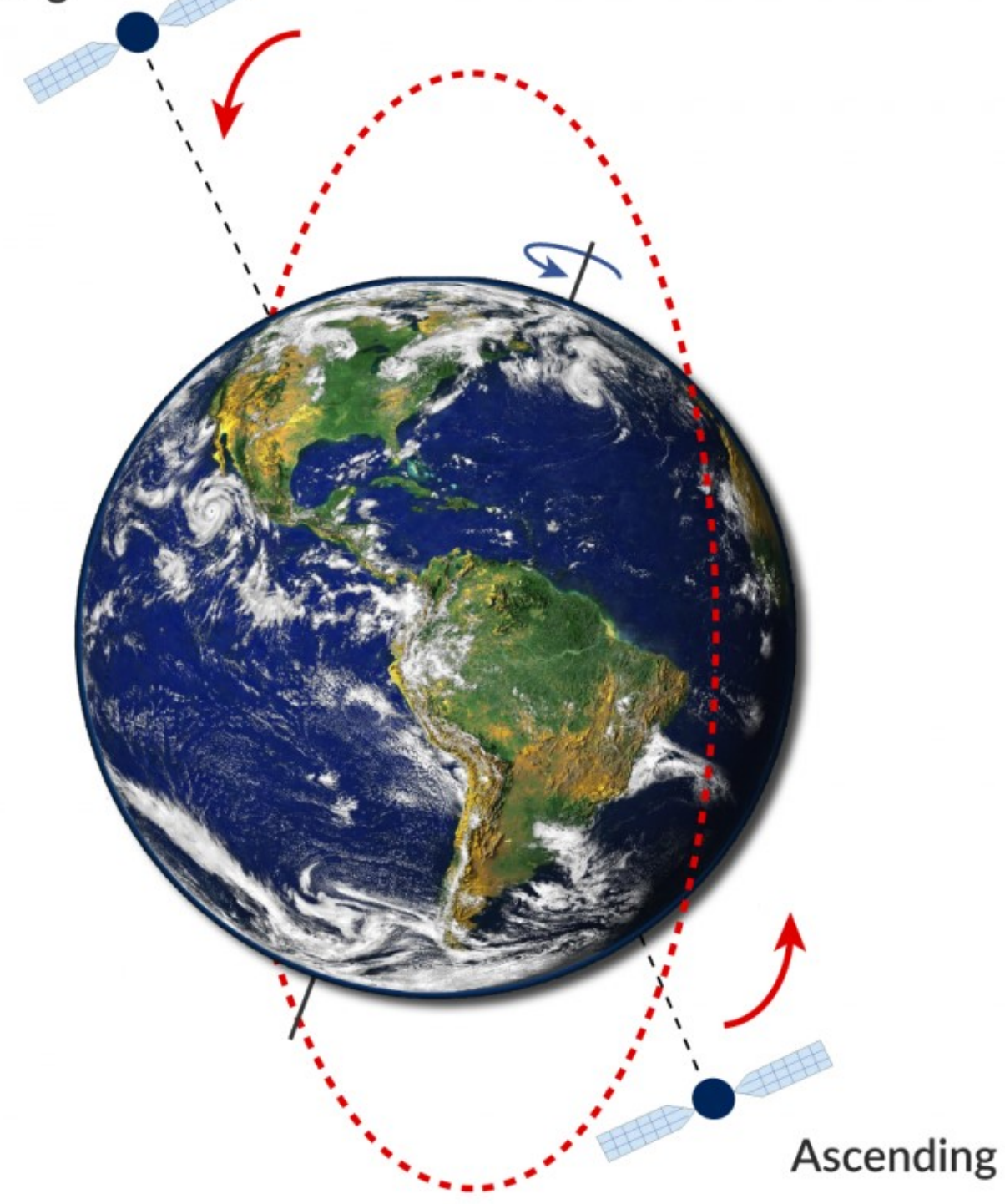

Figure 6: Ascending and descending orbits for spaceborne SAR platforms. Source: SkyGeo 2017. 


\subsection{SAR Images}

SAR sensors transmit and receive the illumination signal, and are known as active sensors. Because of this, and since microwave radiation is not absorbed by water vapour, SAR images can be acquired at day or night, and are not affected by cloud cover. SAR images are influenced, however, by geometrical effects associated with their sidelooking acquisition geometry.

To form a SAR image, the received signal must be processed so that the pixel associated with each ground resolution cell is in the correct location. When processing the received signal, it is assumed that the ground surface is at a constant elevation. SAR mapping is uniform in regions where this assumption is true, i.e. the scale is the same in all directions (NRC 2015). For regions containing topographic features, however, this is not the case. The signal that has been backscattered from a target with a positive elevation is received by the sensor earlier than if this same target was at ground level. The range position is determined by transmission-reception time, and so a target with topography appears to be closer to the sensor than it physically is. Figure 7 shows an example of such a situation. The topographic feature (a rooftop of a house) appears to be to the left of its physical position, i.e. closer to the sensor in the range direction. The converse is also true: an object with a negative elevation appears to be displaced further away from the sensor than it physically is due to the increase in the transmissionreception time. This geometric effect is called foreshortening since objects elevated from the ground appear shorter in a SAR image. The magnitude of foreshortening increases with decreasing incidence angle. Maximum foreshortening occurs when the 
slope of the topographic feature is perpendicular to the incidence angle since the backscatter from the base, slope, and top of the feature are simultaneously received by the sensor. 


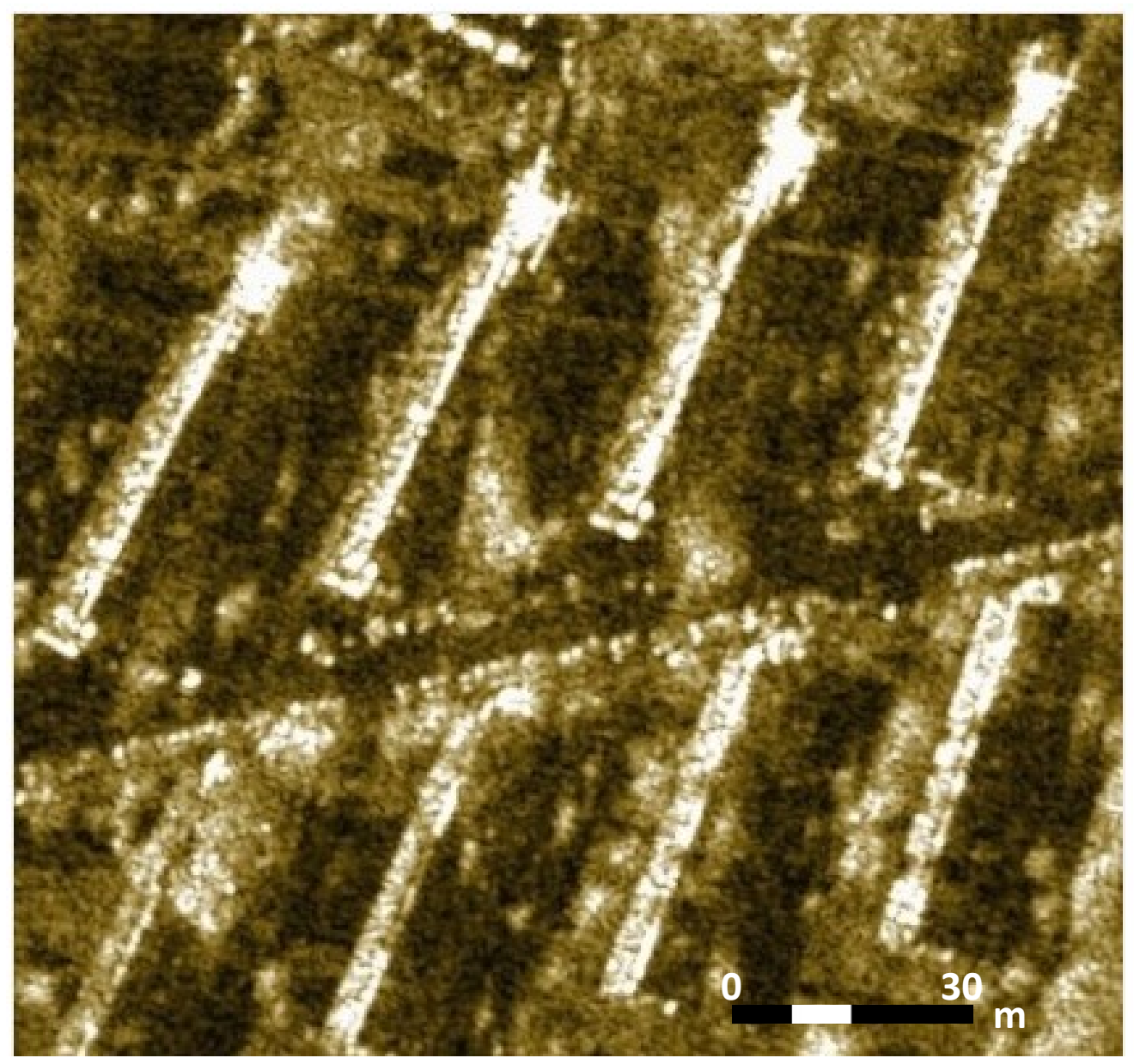

Figure 7: SAR image showing foreshortened rooftops of several houses. Source: SkyRadar 2017. 
Layover is a geometric effect similar to foreshortening. For steeply sloping features with a slope greater than the incidence angle, the transmitted signal will reach the top of the feature before the bottom. This means that the signal backscattered from the top of the feature will reach the sensor before the signal backscattered from the bottom and, in the SAR image, the pixel corresponding to the top of the feature will be closer to the sensor relative to the pixel representing the bottom. The magnitude of layover increases with decreasing incidence angle. Layover results in the misordering of pixels in a SAR image, whereas foreshortening compresses pixels that remain in the correct order. Foreshortening frequently occurs of roofs of houses, which are steeply sloped but not approaching vertical. Layover frequently occurs in high rise buildings due to the tall vertical walls.

Radar shadow is caused by tall vertical features such as buildings. The region behind such a feature (in the LOS direction) is an occluded area since the feature itself blocks the radiation from being transmitted, and therefore no information can be retrieved from the radar shadow zone. Radar shadow occurs when the steepness of the slope on the side facing away from the sensor is greater than the depression angle.

Radar systems measure the distance between the sensor and the target. As a result, SAR images are in Range Doppler Coordinates (RDC) (ESA 2017d), that is, the dimensions of SAR images are in the slant-range and azimuth directions as shown in Figure 8. In RDC, the range direction is compressed relative to the positions of the features on the ground. If the topography is known, images in RDC can be projected into 
Ground Range Coordinates (GRC) such that the range compression is removed. In GRC, the image dimensions are in the range and azimuth direction.

In SAR images both the amplitude and the phase of the received signal is stored. Each pixel in a SAR image is characterised by three values: the row and column number $(x, y)$ that indicates the pixel's range and azimuth position in the image, respectively, and a complex number that contains the amplitude and phase information of the received signal. Images in this format are called Single Look Complex (SLC) images. SLCS are subject to noise, called speckle, which has a granular appearance and greatly reduces image quality. Multilook processing is a method to reduce speckle by averaging neighbouring pixels in a window of a specified size. There are numerous multilook processing algorithms (de Vries 1998), all of which reduce speckle and decrease the geometrical resolution. A multilook-intensity (MLI) image is an SLC image on which multilook processing has been performed. 

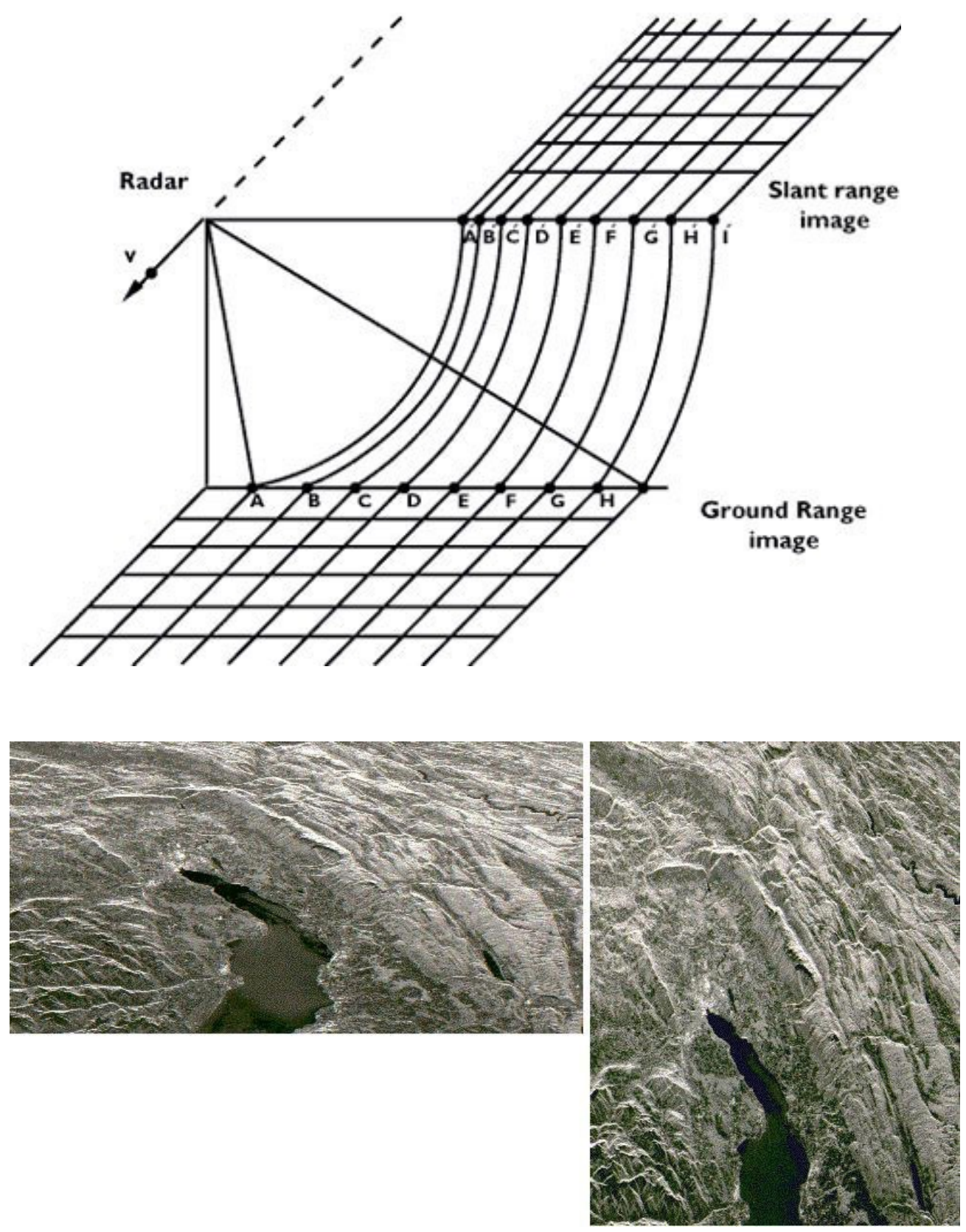

Figure 8: (Top) Diagram showing the range compression in RDC and correction in GRC. (Bottom) A Seasat SAR image of Geneva, Switzerland in RDC (left) and GRS (right). Source: ESA 2017d. 


\section{Chapter 3: SAR Interferometry}

SAR is a coherent imaging system. The phase of each transmitted pulse is defined with respect to a reference, and the phase of the received signal is measured by the SAR sensor with respect to this reference. The coherent nature of the transmitted signal can be exploited to measure small differences in travel path length. An interferogram is an image of the phase difference between two SAR acquisitions; after removal of numerous contributions to SAR image phase, the resulting interferogram is proportional to ground deformation. The theory presented in this chapter is based primarily on the content of: Ferretti et al. 2007a, Ferretti et al. 2007b, Hanssen 2001, and Richards 2007.

\subsection{SAR image phase}

The coherent signal transmitted from a SAR sensor, $x(t)$, can be represented as a complex sinusoid:

$$
\begin{gathered}
x(t)=A \exp \left[i\left(2 \pi F t+\varphi_{T x}\right)\right] \\
0 \leq t \leq \tau
\end{gathered}
$$

where $A$ is the (real-valued) amplitude, $F$ the transmission frequency, $\varphi_{T x}$ the phase, and $\tau$ the pulse duration of the transmitted signal. The transmitted signal propagates at the speed of light, covering the two-way travel distance between the sensor and the ground, $R$, in a time of $2 R / c$. The received signal, $\mathrm{y}(t)$, is then:

$$
y(t)=\hat{A} \rho \exp \left[i\left(2 \pi F\left(t-\frac{2 R}{c}\right)+\varphi_{T x}\right)\right]
$$




$$
\begin{gathered}
y(t)=\hat{A} \rho \exp \left[i\left(2 \pi F t-4 \pi F \frac{R}{c}+\varphi_{T x}\right)\right] \\
y(t)=\hat{A} \rho \exp \left[i\left(2 \pi F t+\varphi_{R x}\right)\right] \\
\frac{2 R}{c} \leq t \leq \frac{2 R}{c}+\tau
\end{gathered}
$$

The frequency of the received signal is unchanged, but the amplitude and received phase, $\varphi_{R x}$, differ. $\hat{A}$ is a complex-valued coefficient that is a function of the transmission amplitude and the gain of the receiver, and $\rho$ is the complex reflectivity of the ground surface (Blahut 2004). The phase of the received signal is shifted by an amount corresponding to the two-way travel distance.

To create a SAR image, $f(x, y)$, where $x$ and $y$ are range and azimuth pixels, respectively, the signal received by the sensor at time $t=t_{0}, y\left(t_{0}\right)$, is processed to remove the transmission frequency and initial phase terms:

$$
\begin{aligned}
f(x, y)= & \widehat{A} \rho(\mathrm{x}, \mathrm{y}) \exp \left(-i \frac{4 \pi F}{c} R(x, y)\right) \\
f(x, y)= & \widehat{A} \rho(\mathrm{x}, \mathrm{y}) \exp \left(-i \frac{4 \pi}{\lambda} R(x, y)\right) \\
& \frac{2 R}{c} \leq t_{o} \leq \frac{2 R}{c}+\tau
\end{aligned}
$$

In equation [7], the received signal (equation [4]) has been applied to each pixel in the SAR image, and the transmission frequency has been rewritten in terms of its wavelength, $\lambda . \widehat{A}$ is constant throughout the image since it depends only on sensor characteristics, but the complex reflectivity of the ground surface and the slant-range distance vary from pixel to pixel. 
The phase, $\varphi_{f}(x, y)$, of each pixel in the SAR image is the argument of $f(x, y)$, such that $\varphi_{f}(x, y)$ is a function of the slant-range distance, sensor and transmitted signal characteristics, and the complex reflectivity of the ground surface:

$$
\varphi_{f}(x, y)=\arg [f(x, y)]=\varphi_{\widehat{A}}+\varphi_{\rho}(x, y)-\frac{4 \pi}{\lambda} R(x, y)
$$

\subsection{Coregistration and interferogram calculation}

The phase of a SAR image pixel contains information regarding the two-way travel distance, and therefore the phase difference between pixels in two SAR images contains information regarding the change in the two-way travel distance, i.e. ground deformation. However, in order to exploit this property of SAR images, corresponding pixels in the two SAR images must be related to the same ground resolution cell. Generally this is not the case for images acquired by satellite-based SAR platforms since the position and orientation of the satellite varies slightly from one pass to the next. The process of resampling one image (the slave), into the geometry of the other (the geometrical master) is called coregistration. Resampling is performed using range and azimuth resampling polynomials, which indicate the required shift of each pixel (in the range and azimuth directions) of the slave image in order for it to overlap with the corresponding pixel in the geometrical master image (Gamma Remote Sensing 2015a). The determination of the coefficients of the resampling polynomials is generally accomplished by first calculating constant range and azimuth offsets from the orbital state vector information associated with each image. Coregistration is then refined by gridding both images into smaller image patches, and subsequently using the two- 
dimensional cross-correlation of these patches to establish additional coefficients in the resampling polynomials (Figure 9).

Two-dimensional cross-correlation is a measure of the similarity of 2, twodimensional functions in terms of the relative displacement between them. If corresponding image patches of the two SAR images are perfectly coregistered, the twodimensional cross-correlation function will have a well-defined peak at zero displacement $(0,0)$, in the same way as an auto-correlation. Conversely, a correlation peak occurring at a non-zero displacement $(I, m)$ indicates that the slave image patch is offset from the geometrical master image patch by / pixels in range and $m$ pixels in azimuth. The range and azimuth resampling polynomials are then calculated, generally by a least squares fit, weighted by the SNR ratio of each patch (Gamma Remote Sensing 2015a). The quality of the image patch correlation is strongly dependant on the amplitude of the pixels; patches containing low intensity regions, such as water bodies or radar shadow, will have broad correlation peaks, reducing the accuracy of the coregistration. 


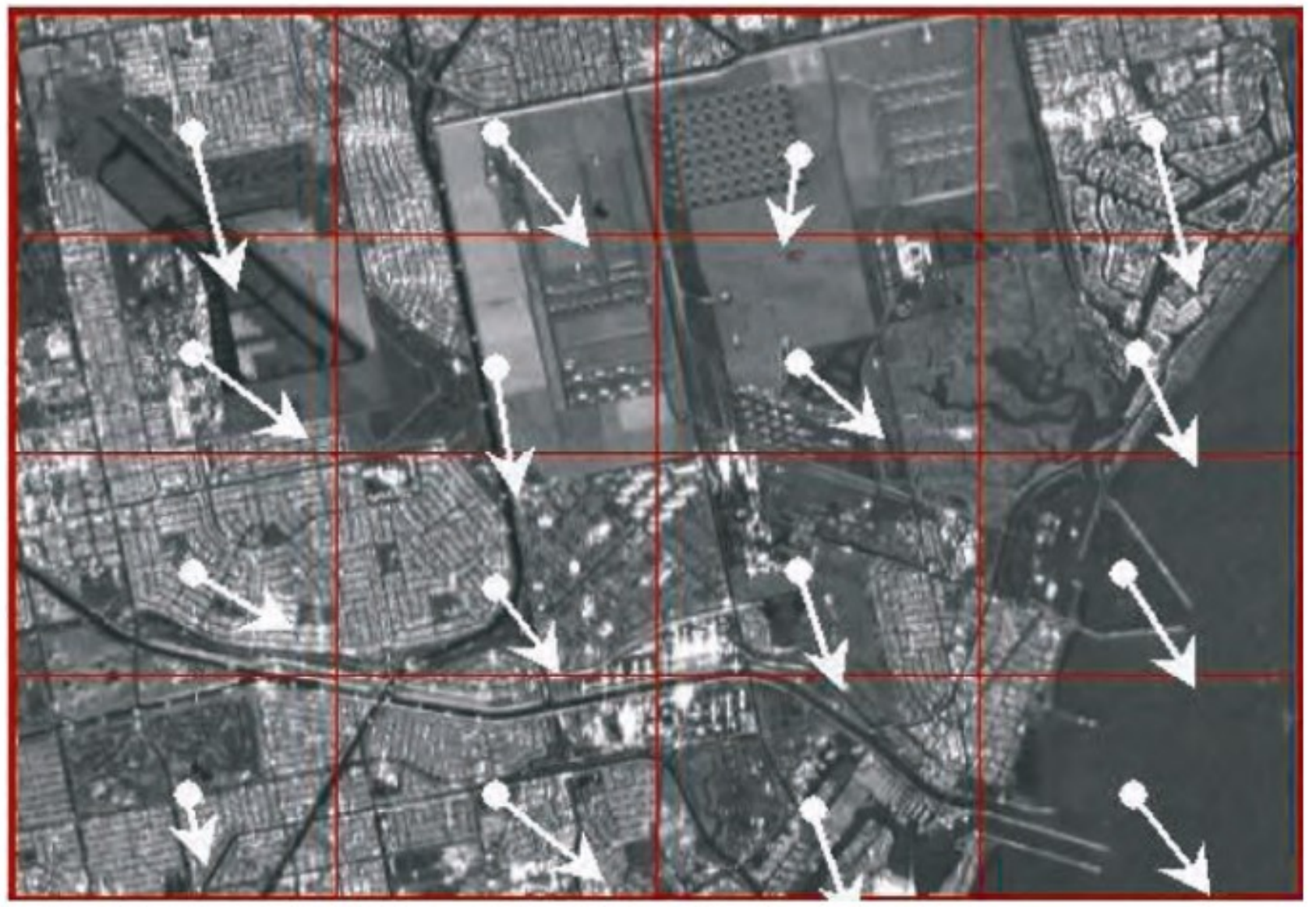

Figure 9: Graphical representation of partitioning a SAR image into patches. The arrows are resampling vectors that indicate the required shift of the slave image patch for it to match the dimensions of the geometrical master. Source: Ferretti et al. 2007b 
The interferogram, $l_{f g}(x, y)$, is calculated by multiplying one SAR image (the interferometric master image), $f(x, y)$, by the complex conjugate of the other (the slave image), $g^{*}(x, y)$ :

$$
\begin{gathered}
I_{f g}(x, y)=f(x, y) g^{*}(x, y) \\
I_{f g}(x, y)=A_{f} A_{g} \exp \left[i\left(\varphi_{f(x, y)}-\varphi_{g(x, y)}\right)\right]
\end{gathered}
$$

Since both SAR images are complex, the resultant interferogram has two channels. The first channel is the real component $\left(A_{f} A_{g}\right)$ which indicates the signal to noise ratio, and may be used to assess the quality of the phase difference measurement. The second channel is the imaginary component, i.e. the interferometric phase difference (IPD) image, $\varphi_{f g}(x, y)$. The IPD image is the main data product of interest in SAR interferometry. Henceforth throughout this thesis and commonly throughout the literature, the term interferogram is taken to refer to $\varphi_{f g}(x, y)$, i.e. the argument of $l_{f g}(x, y):$

$$
\begin{gathered}
\varphi_{f g}(x, y) \stackrel{\text { def }}{=} \arg \left[I_{f g}(x, y)\right] \\
\varphi_{f g}(x, y)=\left(\varphi_{\overparen{A_{f}}}-\varphi_{\overparen{A_{g}}}\right)+\left(\varphi_{\rho_{f}}(x, y)-\varphi_{\rho_{g}}(x, y)\right)-\frac{4 \pi}{\lambda} \Delta R(x, y)+\varepsilon
\end{gathered}
$$

The amplitude phase term is equal to zero since the sensor properties do not change from one pass to the next. If the complex reflectivity of the ground surface has not changed during the interval between acquisitions then its contribution to the IPD is also zero. The IPD, equation [13], is then proportional to the change in slant-range 
distance, $\Delta R$, plus an additional term, $\varepsilon$, which is the combined contribution of numerous error sources:

$$
\varphi_{f g}(x, y)=-\frac{4 \pi}{\lambda} \Delta R(x, y)+\varepsilon
$$

\subsection{IPD contributions: flat Earth, topography, and deformation}

Differences in satellite position at the times of image acquisition correspond to different slant-range distances, even if no ground deformation has occurred (Figure 10). The interferometric baseline, $B$, is defined as the distance between the two satellites in the plane perpendicular to the flight path. The projection of the baseline perpendicular to the slant-range, $B_{\perp}$, is called the perpendicular baseline.

Because of the difference in viewing geometries of two SAR images, the change in slant-range distance term in equation [13] is not only due to deformation, but also because of 1) the increase in slant-range distance with increasing range, and 2) the change in slant-range distance because of topography. When forming a SAR image, a flat reference surface is assumed. The incidence angle increases from near to far range, and therefore the slant-range distance to the flat reference surface also increases from near to far range, as shown in Figure 8 . The increase in slant-range distance with increasing range results in a phase trend, the flat-Earth phase trend, in the range direction of interferograms. The greater the range separation between two pixels, the greater the flat-Earth phase difference between them. Similarly, the slant-range distance to a feature with topography differs when viewed from two different satellite positions (Figure 10). 


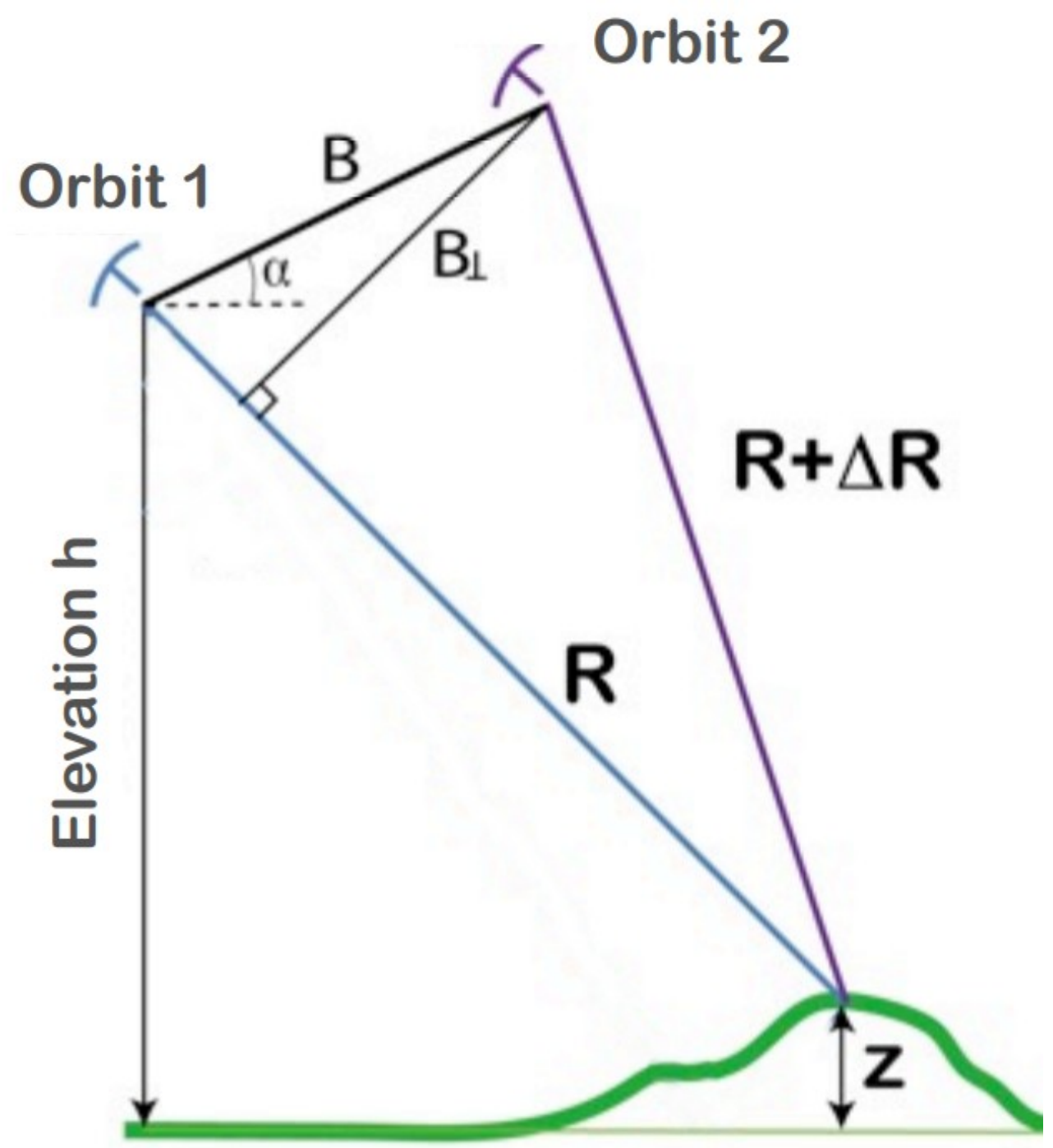

Figure 10: Diagram of the baseline $(B)$, the perpendicular baseline $\left(B_{\perp}\right)$, and the change in slant range distance $(\Delta R)$ due to a flat reference surface and topography (Z) for SAR acquisitions take by satellites at slightly different positions. Source: Doubre 2013 
The interferogram $\varphi_{f g}(x, y)$ is then:

$$
\varphi_{f g}(x, y)=\varphi_{\text {deformation }}(x, y)+\varphi_{F E}(x)+\varphi_{\text {Topo }}(x, y)+\varepsilon
$$

In order to assess ground deformation, contributions from the flat-Earth phase, $\varphi_{F E}(x)$, and the topographic phase, $\varphi_{\text {Topo }}(x, y)$, must be removed. The flat-Earth phase is:

$$
\varphi_{F E}(x)=\frac{-4 \pi}{\lambda} \frac{B_{\perp}}{R \tan (\theta)} \Delta R_{\text {flatEarth }}(x)
$$

Where $\Delta R_{\text {flatEarth }}(x)$ is the difference in slant-range to the flat reference surface as a function of range pixel $(x)$, and $R$ and $\theta$ are the (near range) slant-range distance and incidence angle, respectively. The topographic phase is:

$$
\varphi_{\text {Topo }}(x, y)=\frac{-4 \pi}{\lambda} \frac{B_{\perp}}{R \sin \theta} \Delta z(x, y)
$$

Where $\Delta z$ is the topography with respect to the reference surface, and $B_{\perp}$ the perpendicular baseline. The flat-Earth and topographic phase contributions are subtracted from $\varphi_{f g}(x, y)$ during InSAR processing to create a flattened, differential interferogram, $\varphi_{f g}^{\prime}(x, y)$, from which deformation can be assessed:

$$
\begin{gathered}
\varphi_{f g}^{\prime}(x, y)=\varphi_{f g}(x, y)-\varphi_{F E}(y)-\varphi_{\text {Topo }}(x, y) \\
\varphi_{f g}^{\prime}(x, y)=\varphi_{\text {deformation }}(x, y)+\varepsilon \\
\varphi_{f g}^{\prime}(x, y)=-\frac{4 \pi}{\lambda}\left(\Delta R_{\text {deformation }}(x, y)\right)+\varepsilon
\end{gathered}
$$




\subsection{Atmospheric path delay and phase noise}

The remaining term, $\varepsilon$, in the flattened, differential interferogram is the contribution of atmospheric path delay and phase noise. Atmospheric path delay refers to the variation in two-way travel distance as a result of the differing travel paths taken by two SAR transmissions. The refractive index of the atmosphere changes as a function of humidity, temperature, and pressure, and therefore SAR transmissions experience variable levels of diffraction. For SAR images acquired during stormy weather, the additional two-way travel distance caused by high levels of diffraction can be significant enough to contribute to the interferometric phase (Hanssen 2001). Atmospheric path delay is difficult to remove from interferograms. Some interferogram analysis methods, such as PSInSAR ${ }^{\mathrm{tm}}$ (Ferretti et al. 2000), allow for the estimation and removal of atmospheric effects (if certain assumptions regarding the deformation rate are satisfied), and if large SAR image datasets are used. The major contributions to phase noise are: temporal changes of elementary scatter properties, volume scattering, and instrumental noise of the SAR sensor.

\subsection{Coherence}

SAR image pixels that contain significant phase noise cannot be used to assess ground deformation. The degree to which phase noise is present in an interferogram is estimated by means of the interferometric coherence, $\gamma$. After interferogram flattening and topographic phase correction, $\gamma$ is estimated as (Rosen et al. 2000, Pinto et al. 2013): 


$$
\gamma=\left|\frac{\sqrt{\left\langle f(x, y) g^{*}(x, y)\right\rangle}}{\sqrt{\left\langle f(x, y) f^{*}(x, y)\right\rangle\left\langle g(x, y) g^{*}(x, y)\right\rangle}}\right|
$$

In the above equation, the angular brackets indicate averaging over a specified number of range and azimuth pixels, such that $\gamma$ is an estimate of local coherence. The value of $y$ ranges from 0 to 1 . A value of 0 indicates that the interferometric phase is entirely noise, whereas a value of 1 indicates a complete absence of noise. Ferretti et al. (2007b) draws general conclusions regarding the expected coherence of various regions and features, which are summarized below:

1. Urban structures and areas with exposed rocks maintain a high level of coherence even after several years.

2. Sparsely vegetated areas and agricultural fields generally show high coherence on acquisitions with short time intervals between them (such as the 1-day interval for the tandem ERS mission) but significantly lower coherence after 24 or 35 days. An exception to this is when images separated by an integer number of years are used, particularly for fall-fall or winter-winter acquisitions when vegetation levels are lowest.

3. Forested areas (especially tropical forests) and open water do not maintain coherence even within very short time intervals.

4. Coherence is reduced by local weather events, particularly rain, snow, and very strong winds which can change the complex reflectivity of the ground surface. 


\subsection{Phase unwrapping}

The phase of a SAR image is proportional to the two-way travel distance (equation [8]). However, the phase of the received signal as measured by the SAR sensor is only known to modulo $2 \pi$, i.e. $\varphi_{f}(x, y)$ is restricted to the $(-\pi, \pi)$ interval such that the measured phase is ambiguous by an unknown integer multiple of $2 \pi$. Because of this ambiguity, $\varphi_{f}(x, y)$ is termed the wrapped phase. Therefore the flattened, differential interferogram $\varphi_{f g}^{\prime}(x, y)$ also contains wrapped phase values.

Phase unwrapping is the process of determining the correct integer, $n(x, y)$, such that an unambiguous interferometric phase difference, $\psi_{f g}(x, y)$, that is directly proportional to ground deformation, can be calculated:

$$
\psi_{f g}(x, y)=\varphi_{f g}(x, y)+2 \pi n(x, y)
$$

Numerous phase unwrapping algorithms exist. Almost all of these algorithms are based on the assumption that the unwrapped phase of neighbouring (or nearby) pixels do not differ by more than $\pi$ radians, so that the unwrapped phase is a spatially smooth gradient field. When this assumption is valid, the sum of wrapped interferometric phases around a closed path is equal to zero (i.e. the curl of the gradient field is zero). When this assumption is invalid, usually because of phase noise or rapid deformation, the sum of wrapped interferometric phases around a closed path is not equal to zero, and a discontinuity (or residue) occurs. Stated differently, the assumption made by phase unwrapping algorithms is that the discrete derivative (the phase gradient 
between neighbouring pixels) of the wrapped phase, $\Delta \psi(x, y)$, is equal to the discrete derivative of the unwrapped phase $\Delta \varphi(x, y)$ :

$$
\Delta \psi(x, y)=\Delta \varphi(x, y)
$$

The phase unwrapping algorithm implemented in this thesis, the Minimum Cost Flow (MCF) algorithm (Flynn et al. 1997, Constantini et al. 1998), seeks to minimize phase discontinuities by minimizing the cost function, $C$, (Ghiglia D.C et al. 1998, Chen et al. 2000):

$$
C=\left\{\sum_{x, y} w_{x, y}^{r}\left|\Delta^{r} \psi_{x, y}-\Delta^{r} \varphi_{x, y}\right|+w_{x, y}^{a} \sum_{x, y}\left|\Delta^{a} \psi_{x, y}-\Delta^{a} \varphi_{x, y}\right|\right\}
$$

In equation [23], $x$ and $y$ are the range and azimuth pixel coordinates, respectively, $w_{x, y}$ are weighting factors (usually coherence), and $\Delta \psi(x, y)$ and $\Delta \varphi(x, y)$ are the phase gradients in the range $(r)$ and azimuth $(a)$ directions. The value of $\Delta \psi(x, y)$ that minimizes the cost function corresponds to the lowest possible values of $n(x, y)$ to make the unwrapped phase consistent with the wrapped phase. Assuming atmospheric and noise contributions to phase are small, $\psi_{f g}(x, y)$ is directly proportional to ground deformation in the same way as described by equation [19].

\subsection{Time Series Analysis}

Time series analysis refers to a collection of advanced InSAR processing techniques that allow for the combination of multiple interferograms to establish a deformation time series. There are numerous algorithms that perform time series analysis, with no single algorithm being optimal in all circumstances. The only common 
characteristic of time series analysis algorithms is that they all produce a first order estimate of deformation rates. The amount of interferograms required, whether these interferograms are generated from a single or multiple interferometric masters, whether the unwrapped or wrapped phase measurements are utilized, and whether the linear or non-linear deformation rate is required, among other properties, are all considerations in determining what time-series algorithm (or combination) is used. Osmanoglu et al. (2016) and Crosetto et al. (2016) summarize recent time series analysis trends. In this project, the GAMMA SAR and Interferometry Software Packages multibaseline algorithm (Gamma Remote Sensing 2015c) is used.

The multi-baseline algorithm maximizes the exploitation of small datasets by using multiple interferometric masters to generate a large interferogram suite. This algorithm provides estimates of time series deformation rates using a weighted least-squares approach to minimize the sum of squared weighted residual phases. Singular value decomposition is used to derive the least squares solution. The residuals are the differences between input phases (the observations) and the differential phases derived from the time series solution. The time series is smoothed to help reduce the effect of abrupt changes due to atmospheric path delay in some acquisitions. Further details of the multi-baseline algorithm can be found in Gamma Remote Sensing (2015c). 


\section{Chapter 4: Geological Setting, Site Selection Criteria,}

\section{Data Description}

Two study areas in Eastern Ontario have been selected for study. The first study area is Cornwall, Ontario, Canada (Figure 11). This study area was selected based off the selection criteria described in the next subsection (Section 4.1.), and analysed with the RADARSAT-2 SAR data described in a subsequent section (Section 4.5.1.). A significantly larger study area (Figure 12) which includes Cornwall, Ontario, but additionally the Ottawa and East Hawkesbury regions of Eastern Ontario was selected for additional analysis with the Sentinel-1 SAR data described in Section 4.5.2. The extent of this larger study area was selected based on the considerations described in Section 5.2., Sentinel1 Methodology.

\subsection{Site Selection Criteria}

Cornwall, Ontario, Canada has been selected for study with RADARSAT-2 data. The study area was selected based on the following general criteria: (1) availability of suitable RADARSAT-2 SAR images; (2) presence of a variety of urban structures and linear transportation infrastructure; (3) evidence of a potential geohazard; (4) availability of ancillary geological, geodetic, and meteorological ground-truth data. Specific considerations for each criterion are described:

1. In terms of the characteristics of the SAR data itself, three criteria that the RADARSAT-2 data must fulfill were established. RADARSAT-2 data was required 
to be in an archived data stack, coeval with Sentinel-1A observations, and at a moderate-high resolution. Deformation monitoring with InSAR requires a large data stack. Due to the long time required to gather such a data stack with a platform with a 24 day repeat period, using an archived dataset was necessary. In order to directly compare Sentinel-1A and RADARSAT-2 observations, the datasets from these two platforms should be at least partially overlapping in time. Linear infrastructure elements such as railway lines and highways are on the order of $10 \mathrm{~m}$ wide, and for this reason moderate to high resolution data was assumed to be most suitable. RADARSAT- 2 datasets with a resolution coarser than $8 \mathrm{~m}$ (i.e. the nominal resolution of the various Fine beam modes (MDA 2016a)) were not considered based off this assumption.

2. Numerous types of linear transportation infrastructure exist: railways, highways, streets, bridges etc., and these elements vary significantly in their morphology as well as in their construction materials. In order to quantify how these properties affect the ability of InSAR to monitor these elements, a variety of such features is required.

3. In order to quantify the impact of geohazards on linear infrastructure, a geohazard must somewhere intersect infrastructure elements. In Eastern Ontario, lateral spreading and differential settlement in Leda clay is the primary geohazard. In order for a location to be considered for analysis it was required that there be Leda clay deposits and evidence of previous mass movements. 
Locations that did not meet these criteria were only considered if other specific known geohazards were present.

4. In addition to SAR imagery, availability of ancillary data was also considered during the site selection process. A Digital Elevation Model (DEM) or Digital Surface Model (DSM) is required for differential interferometry. This DEM should be at a similar resolution to the SAR dataset, and for areas experiencing rapid deformation it should be contemporaneous with the SAR dataset. In order to validate InSAR derived deformation, a source of ground-truthing data was also required. This data must be deformation measured with a well-established deformation monitoring technique such as leveling or GPS, and must be of a feature that would likely remain coherent throughout a SAR stack. Furthermore, the measured deformations must have been occurring over the same time period as the SAR data. In addition to this, information that would aid interpretation is desirable. This includes, but is not limited to, weather records to help establish atmospheric effects, and a map of the surficial geology.

\subsection{Cornwall Ontario, Canada}

Cornwall, Ontario, Canada best satisfies the above criteria. The construction of the Seaway International Bridge connecting Cornwall, Ontario, Canada to Massena, New York, USA is currently being monitored with InSAR. This has established an archived data set consisting of more than 75 high resolution (approximately $3 \mathrm{~m}$ ) scenes coinciding

with Sentinel-1A observations, satisfying criterion (1). Being an urban center, Cornwall 
contains numerous urban structures and common infrastructure such as streets and a highway (highway 401). Additionally, a railway line, a hydro-electric dam (R.H. Saunders Generating Station), a dam access road with embankments, and a bridge (the Seaway International Bridge) are present, satisfying criterion (2). As seen from the surficial geology map (Figure 13), Cornwall contains a significantly smaller fraction of Leda Clay than in the Ottawa area. However, isolated pockets occupying an abandoned stream channel in the center of the city, and a larger pocket in the east end of the city are present, satisfying criterion (3). Finally, criterion (4) is also satisfied in Cornwall, Ontario: the construction of the Seaway International Bridge has been additionally monitored with traditional techniques allowing access to third-party ground-thruthing data, and a recent (circa 2012) high resolution DSM is available (D. Lalonde, personal communication). As with most locations in Eastern Ontario, a map of surficial geology (Figure 13) and weather records (Tables 1 and 2) are available for Cornwall.

\subsubsection{Case Studies}

The specific features for which InSAR monitoring was attempted are described below:

1. R.H. Saunders Generating Station Access Road: The R.H. Saunders Generating Station is a hydro-electric power dam constructed in 1954-1958, situated between Cornwall, Ontario and Barnhart Island, Massena, New York. The dam impounds the Saint Lawrence River, and created the Lake Saint Lawrence reservoir. Service to the dam is done primarily via an access road that runs immediately adjacent to the Saint Lawrence River. This access road was built on 
a stone embankment to protect it and the adjacent areas from high water levels in Lake Saint Lawrence.

2. CN Railway Line: A two track portion of the CN Railway line runs east-west through Cornwall, just south of highway 401. This portion of the railway line is predominantly on sand and diamicton, but also crosses a Leda clay pocket. A larger stretch of the railway line, approximately $7.48 \mathrm{~km}$, is contained within the descending mode acquisitions than within the ascending acquisitions, which cover approximately $3.78 \mathrm{~km}$ of track.

3. Two specific sections of city streets have been selected: the intersection of Emma Ave and Cumberland St, and the approximately $100 \mathrm{~m}$ long stretch of Saunders Rd just off of Power Dam Drive. On Thursday April 6 2017, the EmmaCumberland intersection was closed due collapse of the road surface. Beneath this intersection lies a culvert, which directs water flowing from the Boal's Drain floodplain. Due to the large amount of precipitation in spring 2017 (see Table 1), this culvert failed, and subsequently the road surface collapsed. Similarly, in spring to summer 2016 Saunders Rd was closed after it was discovered that a culvert had corroded, and the soil between the culvert and road surface had been washed away. In contrast to the Emma-Cumberland intersection, on Saunders Rd the road surface remained intact, but was too unstable to support traffic. 
4. St. John's Presbyterian Church: this church was constructed in 1888-1889, and is located at 28 Second St East. It was selected for monitoring since it is a historic building located very close to the former site of the Knox-St. Paul's United Church, which was demolished in 2009. The Knox-St. Paul's United Church was erected in 1884 at 108 Second Street East, and built with a timber raft foundation. This church had been sinking and experiencing structural issues for several decades prior to its demolition. The St. John's Presbyterian Church is built with the same type of timber raft foundation as the Knox-St. Paul's United Church.

\subsection{Subset of Eastern Ontario}

A second, significantly larger area (Figure 12), which includes the study site of Cornwall Ontario, but additionally the Ottawa and East Hawkesbury regions of Eastern Ontario, has been selected for additional analysis. This larger region was selected partially because the Ottawa - East Hawkesbury axis contains significantly more Leda clay deposits and therefore a higher lateral spreading risk than Cornwall, but additionally because of the Sentinel-1 processing considerations detailed in Section 5.2.

\subsection{Geological Setting and Geohazards in Eastern Ontario}

The study areas (Figures 11 and 12) are located entirely within the expansive deposits of marine soils of the Saint Lawrence Lowlands, which were deposited during the invasion of the Champlain sea, a temporary inlet of the Atlantic Ocean that existed from approximately 12,000 to 9,500 years ago (Quinn 2009). The Saint Lawrence 
Lowlands are relatively low lying compared to the metamorphic bedrock of the Grenville Province to the north of the Ottawa River, and Ordovician-Silurian aged sedimentary rocks of the Appalachian Orogen to the south of the Saint Lawrence River. The topography is generally relatively flat, sloping gently to the north and south towards the Ottawa and Saint Lawrence rivers. Numerous smaller rivers cut the landscape and are tributaries to the Ottawa and Saint Lawrence rivers.

The basement of the St. Lawrence Lowlands consists of igneous and metamorphic Precambrian rocks uncomfortably overlain by Paleozoic age sedimentary rocks (Quinn 2009, Morton 2015). These Paleozoic sedimentary rock layers range from sandstones and dolostones deposited and lithified during the Upper Cambrian to Middle Ordovician Periods, to limestones, siltstones, and shales deposited and lithified during the Middle to Upper Ordovician Period.

Overlying these Paleozoic sedimentary rocks is unconsolidated overburden of varying thickness, consisting predominantly of marine sediments; it is this overburden, specifically the clay fraction, which is responsible for the high lateral spreading risk and geotechnical issues in the region (Bobrowsky et al. 2012, Quinn 2009). Numerous glaciations have occurred since the formation of the Paleozoic bedrock, with the most recent largely overprinting the evidence of earlier ones. The advance and subsequent retreat of the Laurentide ice sheet during the most recent glaciation, the Wisconsinan, is predominantly responsible for the deposition of the present day overburden and topographic features in this region. 
During the most recent advance of the Laurentide ice sheet, the weight of the glacier caused subsidence of the present day Saint Lawrence Lowlands to below sea level. During this time an ice dam formed, temporarily blocking the influx of water from the Atlantic Ocean (Karrow 1965). As the Laurentide ice sheet retreated, its glacial meltwater formed two proglacial lakes on its Western and Eastern fronts: Lake Agassiz and Lake Ojibway, respectively. Approximately 12,000 years ago, the Laurentide ice sheet made its final retreat and the ice dam blocking the Atlantic Ocean melted, causing the rapid inundation of the low lands with saline ocean water. This water mixed with the freshwater draining from Lake Ojibway, forming a brackish water body. For a time, this water body remained in contact with the still retreating Laurentide ice sheet resulting in a brackish glacio-marine environment; the Champlain Sea. This sea remained in the inlet for about 2,500 years, during which time the deposition of marine sediments, broadly classified by Karrow (1965) as Leda clay and Saxicava sand, occurred. Since the deposition of these sediments occurred in a brackish marine environment the Leda clay developed a relatively stiff, meta-stable, flocculated structure with saline pore fluids.

Following the final retreat of the Laurentide ice sheet, isostatic rebound occurred, draining the Champlain Sea into the Atlantic Ocean, and resulting in relatively flat clay plains with high standing areas composed of sand and gravel representing beaches of the Champlain Sea. Isostatic rebound also allowed Lake Ojibway and Lake Agassiz to form flow channels through the Ottawa Valley. This developed into the relatively rapidly flowing Proto-Ottawa and Saint Lawrence Rivers which scoured the flow channel of the fine grained marine sediments and replaced them with coarser freshwater fluvial 
deposits of sand and gravel. These presently dry channels are particularly evident near Ottawa. Since the drainage of the Champlain Sea, a network of rivers and streams has formed due to the eroded marine sediments. These marine sediments, particularly the Leda clay, have very low permeability (coefficients of permeability range from $5.52 \times 10^{-10}$ and $1.09 \times 10^{-6} \mathrm{~cm} / \mathrm{s}$. Athir 2014, p.114) resulting in significant surface run off (Karrow 1965). The isostatic rebound of the Saint Lawrence Lowlands drastically altered the hydrogeological conditions of the present day overburden; the originally saline pore fluids of the Leda clay were replaced with freshwater pore fluids. The result is that the stiff, flocculated structure of undisturbed Leda clay can rapidly change to a very weak, dispersed structure when remoulded. This sensitivity of Leda clay is the cause of the high lateral spreading risk in Eastern Ontario. 


\subsection{Data}

Two SAR datasets were used in this project: RADARSAT-2 Spotlight mode, and Sentinel-1A Interferometric Wide (IW) mode. The RADARSAT-2 Spotlight data was provided by the CSA through the SOAR-Education program. This data is commercially available from Macdonald Dettwiler and Associates for \$CAD 6,000 per scene (MDA 2016b). Sentinel-1A IW images are freely available to the public via the European Space Agency's Copernicus program's Scientific Data Hub ${ }^{1}$.

\subsubsection{RADARSAT-2 Spotlight mode}

The spotlight beam mode is the highest spatial resolution SAR data available from the RADARSAT-2 platform (MDA 2016a). In contrast to the other available RADARSAT-2 modes, with Spotlight mode the beam is electronically steered to linger on the ground resolution cell, allowing the image to be processed to higher azimuth resolutions. The nominal azimuth resolution for Spotlight mode SLC data is $0.8 \mathrm{~m}$, and the relative phase error is less than $3^{\circ}$ (MDA 2016a). Electronic beam steering illuminates a smaller portion of the ground compared to other beam modes, resulting in relatively small swaths with nominal dimensions of $18 \mathrm{~km}$ in ground range and $8 \mathrm{~km}$ in azimuth.

In this project, 34 products from two RADARSAT-2 Spotlight beam modes were used: 19 SLA24 SLC images, and 15 SLA74 SLC images (Table 1). The SLA24 dataset has a nominal near- and far-range incidence angle of $46.8^{\circ}$ and $47.7^{\circ}$, respectively. This

\footnotetext{
${ }^{1}$ https://scihub.copernicus.eu/
} 
corresponds to a nominal near- and far-range resolution of $2.2 \mathrm{~m}$ and $2.1 \mathrm{~m}$, respectively (MDA 2016a). The SLA74 dataset was acquired with a nominal near- and far-range incidence angle of $24^{\circ}$ and $25.5^{\circ}$, and nominal near- and far-range resolution of $3.9 \mathrm{~m}$ and $3.7 \mathrm{~m}$ (MDA 2016a). The SLA24 data used in this project was acquired in a descending orbit with all acquisitions taken between 10:57:31.8437 and 10:57:11.2406 coordinated universal time (UTC). The SLA74 data used in this project was acquired in an ascending orbit with all acquisitions taken between 22:42:35.8500 and 22:42:14.9863 UTC. Both the SLA24 and SLA74 images were acquired using HH polarization (horizontal transmit and horizontal receive). 
Table 1: RADARSAT-2 acquisition dates, beam modes, and weather conditions. SLA74 images were acquired in an ascending orbit, while SLA24 images were acquired in a descending orbit.

\begin{tabular}{|c|c|c|c|}
\hline Date & Beam Mode & $\begin{array}{l}\text { Snow on ground } \\
\text { in Cornwall }(\mathrm{cm})\end{array}$ & $\begin{array}{l}\text { Total daily precipitation } \\
\text { in Cornwall }(\mathrm{mm})\end{array}$ \\
\hline 2015-03-03 & SLA74 & 60.0 & 5.0 \\
\hline 2015-03-21 & SLA24 & 38.0 & 0.0 \\
\hline $2015-03-27$ & SLA74 & 29.0 & 1.0 \\
\hline 2015-04-14 & SLA24 & 0.0 & 0.0 \\
\hline $2015-04-20$ & SLA74 & 0.0 & 26.5 \\
\hline 2015-05-08 & SLA24 & 0.0 & 0.0 \\
\hline 2015-06-01 & SLA24 & 0.0 & 1.0 \\
\hline $2015-06-07$ & SLA74 & 0.0 & 8.4 \\
\hline $2015-06-25$ & SLA24 & 0.0 & 0.0 \\
\hline $2015-07-25$ & SLA74 & 0.0 & 3.8 \\
\hline $2015-08-12$ & SLA24 & 0.0 & 0.0 \\
\hline $2015-08-18$ & SLA74 & 0.0 & 0.0 \\
\hline $2015-09-05$ & SLA24 & 0.0 & 0.0 \\
\hline 2015-09-11 & SLA74 & 0.0 & 1.0 \\
\hline $2015-10-23$ & SLA24 & 0.0 & 0.0 \\
\hline 2015-11-16 & SLA24 & 0.0 & 0.0 \\
\hline $2016-02-20$ & SLA24 & 17.0 & 9.8 \\
\hline $2016-02-26$ & SLA74 & 4.0 & 0.0 \\
\hline $2016-03-15$ & SLA24 & 0.0 & 1.2 \\
\hline 2016-03-21 & SLA74 & 0.0 & 0.0 \\
\hline 2016-04-08 & SLA24 & 0.0 & 0.0 \\
\hline 2016-05-02 & SLA24 & 0.0 & 2.0 \\
\hline 2016-05-08 & SLA74 & 0.0 & 0.0 \\
\hline 2016-05-26 & SLA24 & 0.0 & 0.0 \\
\hline 2016-06-01 & SLA74 & 0.0 & 2.0 \\
\hline 2016-06-19 & SLA24 & 0.0 & 0.0 \\
\hline 2016-06-25 & SLA74 & 0.0 & 0.0 \\
\hline $2016-07-13$ & SLA24 & 0.0 & 0.0 \\
\hline 2016-07-19 & SLA74 & 0.0 & 0.0 \\
\hline 2016-08-06 & SLA24 & 0.0 & 0.0 \\
\hline $2016-08-12$ & SLA74 & 0.0 & 16.4 \\
\hline $2016-08-30$ & SLA24 & 0.0 & 3.0 \\
\hline 2016-09-05 & SLA74 & 0.0 & 0.0 \\
\hline $2016-09-23$ & SLA24 & 0.0 & 2.0 \\
\hline
\end{tabular}




\subsubsection{Sentinel-1A: Interferometric Wide}

The Sentinel-1A platform operates in a pre-planned acquisition schedule, i.e. data acquisition is pre-determined, and not commercially requested (ESA 2017c). The IW beam mode is the main imaging mode over land. IW images are generated using the Terrain Observation and Progressive Scans SAR (TOPSAR) acquisition mode. In the TOPSAR mode, the full image is constructed from three subswaths, with each subswath being $1 / 3$ of the full image in the range direction, and the full azimuth length. Each subswath is made up of (up to) ten smaller sections called bursts. Successive bursts contain a small overlapping region to allow them to be mosaicked, and adjacent subswaths also contain a slight overlap for the same reason. As described in Section 5.2., Sentinel-1 Methodology, the burst IW image resulting from the TOPSAR mode places very strict requirements on coregistration.

The TOPSAR technique involves electronically steering the radar beam in both the azimuth and range directions to maximize the time each burst region is illuminated; the radar beam is electronically steered back and forth in the azimuth direction for each burst, and cyclically switched amongst the three sub-swaths. The advantage of this illumination strategy is being able to generate very large swaths, at moderate resolution, with a near uniform signal to noise ratio. For IW SLC images the nominal ground-range width is $251.8 \mathrm{~km}$, and the nominal azimuth resolution is $21.7 \mathrm{~m}$ (ESA 2017c). The nominal near-range (i.e. subswath 1) and far-range (i.e. subswath 3) incidence angles are $32.9^{\circ}$ and $43.1^{\circ}$, respectively, corresponding to nominal near- and far-range resolutions of $2.7 \mathrm{~m}$ and $3.5 \mathrm{~m}$. 
In this project, $33 \mathrm{IW}$ images from 28 dates were used, with all IW images acquired in an ascending orbit, with both VV (vertical transmit and vertical receive) and VH (vertical transmit and horizontal receive) polarizations. In most cases, the study area fell entirely within a single IW image. For a few dates (2015-08-15, 2017-02-17, 2017-0313, 2017-03-25, 2017-04-06), however, due to minor variances in the satellite's orbit, the full area of interest was not included within a single acquisition. For these dates, two images, one with a slightly earlier acquisition time than the subsequent, were downloaded and concatenated to cover the full area of interest. This procedure is described in more detail in Section 5.2. Table 2 lists all IW images used in this project, as well as relevant weather conditions at the time of acquisition. 
Table 2: Sentinel-1 IW acquisition dates, acquisition times, and weather conditions. All acquisitions are SLC IW images.

\begin{tabular}{|l|l|l|l|l|l|}
\hline Date & Time (UTC) & $\begin{array}{l}\text { Snow on } \\
\text { ground in } \\
\text { Cornwall } \\
\text { (cm) }\end{array}$ & $\begin{array}{l}\text { Total daily } \\
\text { precip. } \\
\text { Cornwall } \\
\text { (mm) }\end{array}$ & $\begin{array}{l}\text { Snow on } \\
\text { Ground } \\
\text { Ottawa } \\
\text { (cm) }\end{array}$ & $\begin{array}{l}\text { Total daily } \\
\text { precip. } \\
\text { Ottawa (mm) }\end{array}$ \\
\hline $2015-07-22$ & 225153.8937 & 0.0 & 1.2 & 0.0 & 0.0 \\
\hline $2015-08-15$ & 225146.9721 & 0.0 & 0.0 & 0.0 & 0.0 \\
\hline $2015-08-15$ & 225211.7950 & 0.0 & 0.0 & 0.0 & 0.0 \\
\hline $2015-08-27$ & 225155.7888 & 0.0 & 0.0 & 0.0 & 0.0 \\
\hline $2015-09-08$ & 225156.2555 & 0.0 & 0.0 & 0.0 & 0.0 \\
\hline $2015-09-20$ & 225156.5376 & 0.0 & 0.0 & 0.0 & 0.0 \\
\hline $2015-10-02$ & 225156.7753 & 0.0 & 0.0 & 0.0 & 0.0 \\
\hline $2015-10-26$ & 225156.8045 & 0.0 & 0.0 & 0.0 & 0.0 \\
\hline $2015-11-19$ & 225150.9623 & 0.0 & 4.0 & 0.0 & 6.2 \\
\hline $2015-12-13$ & 225150.1842 & 0.0 & 2.2 & 0.0 & 3.0 \\
\hline $2016-03-30$ & 225151.8122 & 0.0 & 6.2 & 0.0 & 14.6 \\
\hline $2016-04-23$ & 225158.2583 & 0.0 & 0.0 & 0.0 & 0.0 \\
\hline $2016-06-10$ & 22523.6878 & 0.0 & 0.0 & 0.0 & 3.6 \\
\hline $2016-07-28$ & 22526.2575 & 0.0 & 0.0 & 0.0 & 0.0 \\
\hline $2016-09-26$ & 22520.7057 & 0.0 & 0.0 & 0.0 & 1.2 \\
\hline $2016-10-08$ & 22520.6932 & 0.0 & 11.4 & 0.0 & 4.2 \\
\hline $2016-10-20$ & 22520.5743 & 0.0 & 15.0 & 0.0 & 22.4 \\
\hline $2016-11-01$ & 22520.6509 & 0.0 & 0.0 & 0.0 & 0.2 \\
\hline $2016-11-13$ & 22520.5056 & 0.0 & 0.0 & 0.0 & 0.0 \\
\hline $2016-11-25$ & 22520.3326 & 0.0 & 3.2 & 4.0 & 0.8 \\
\hline $2016-12-07$ & 225159.8989 & 13.0 & 0.0 & 3.0 & 0.0 \\
\hline $2016-12-19$ & 225159.4387 & 16.0 & 0.0 & 21.0 & 0.0 \\
\hline $2016-12-31$ & 225159.0129 & 10.0 & 4.0 & 18.0 & 8.6 \\
\hline $2017-01-12$ & 225157.5248 & 0.0 & 2.0 & 26.0 & 0.4 \\
\hline $2017-01-24$ & 225157.2815 & 0.0 & 4.0 & 20.0 & 6.0 \\
\hline $2017-02-05$ & 225157.0453 & 7.0 & 3.0 & 24.0 & 1.2 \\
\hline $2017-02-17$ & 225140.1832 & 35.0 & 0.0 & 60.0 & 0.0 \\
\hline $2017-02-17$ & 22525.0081 & 35.0 & 0.0 & 60.0 & 0.0 \\
\hline $2017-03-13$ & 225140.3481 & 0.0 & 1.0 & 11.0 & 0.0 \\
\hline $2017-03-13$ & 22525.1731 & 0.0 & 1.0 & 11.0 & 0.0 \\
\hline $2017-03-25$ & 225140.5710 & 18.0 & 0.0 & 22.0 & 0.0 \\
\hline $2017-03-25$ & 22525.3939 & 18.0 & 0.0 & 22.0 & 0.0 \\
\hline $2017-04-06$ & 225141.0342 & 0.0 & 46.0 & 0.0 & 27.8 \\
\hline $2017-04-06$ & 22525.8591 & 0.0 & 46.0 & 0.0 & 27.8 \\
\hline & & & & & \\
\hline & & & 0.0 & \\
\hline
\end{tabular}




\subsubsection{Digital Surface Models}

Two DSMs are used in this project. For the analysis of Cornwall, a high resolution LiDAR derived DSM is used (Figure 11). The point cloud of LiDAR elevations was measured in 2012 with approximately $1 \mathrm{~m}$ point spacing. Using ArcMap, this point cloud was interpolated to a $3 \mathrm{~m}$ raster grid and projected into NAD 1983 UTM Zone 18N coordinates. The LiDAR point cloud was provided through personal communication with the City of Cornwall.

For the secondary analysis of Eastern Ontario, a significantly larger, coarser DSM is used (Figure 12). This DSM is a section of Natural Resources Canada's Canadian Digital Surface Model mosaic ${ }^{2}$. This 0.75 arc-second (approximately 20 m resolution) DSM is a derived product of the Shuttle Radar Topography Mission (SRTM) DSM, which acquired data at 1 arc-second (approximately $30 \mathrm{~m}$ resolution). Elevations were measured in 2000. Using ArcMap, the DSM was projected into NAD 1983 UTM Zone 18N coordinates.

\footnotetext{
${ }^{2}$ http://geogratis.gc.ca/site/eng/extraction
} 


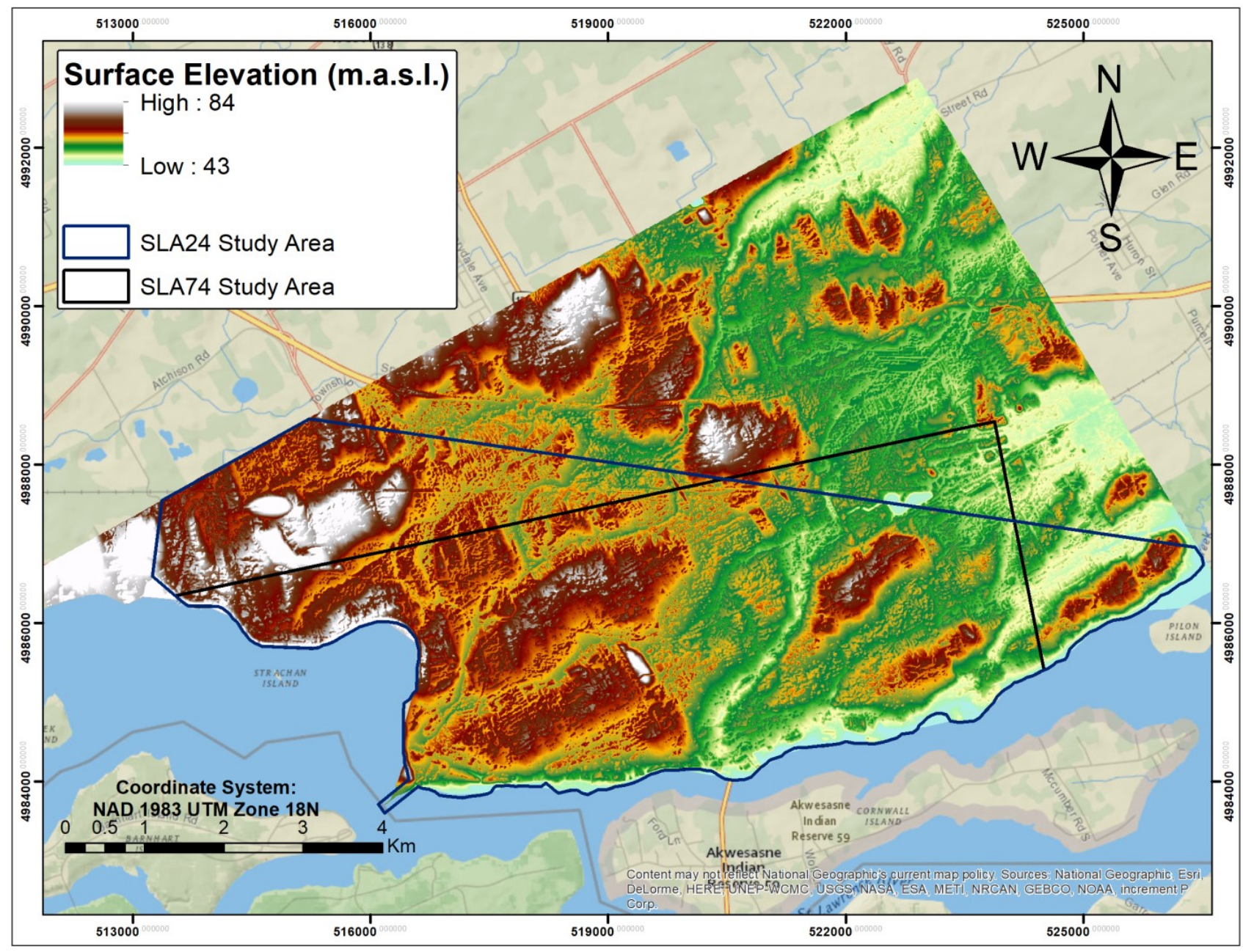

Figure 11: LiDAR Derived 3 m DSM of Cornwall Ontario, and the RADARSAT-2 SLA24 and SLA74 study areas. 


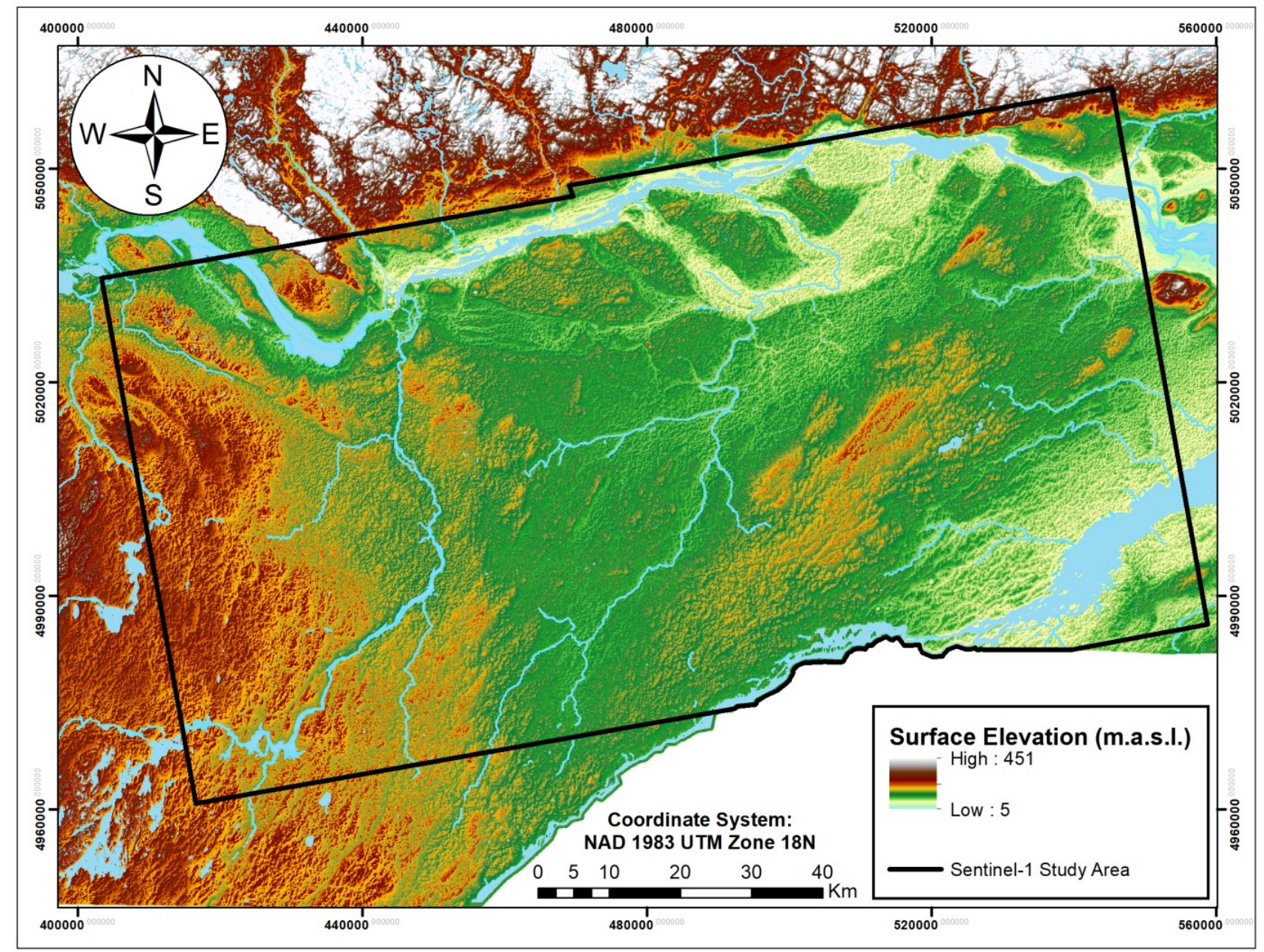

Figure 12: Subset of Natural Resources Canada's 20 m Canadian DSM mosaic, and the Sentinel-1 study area. 


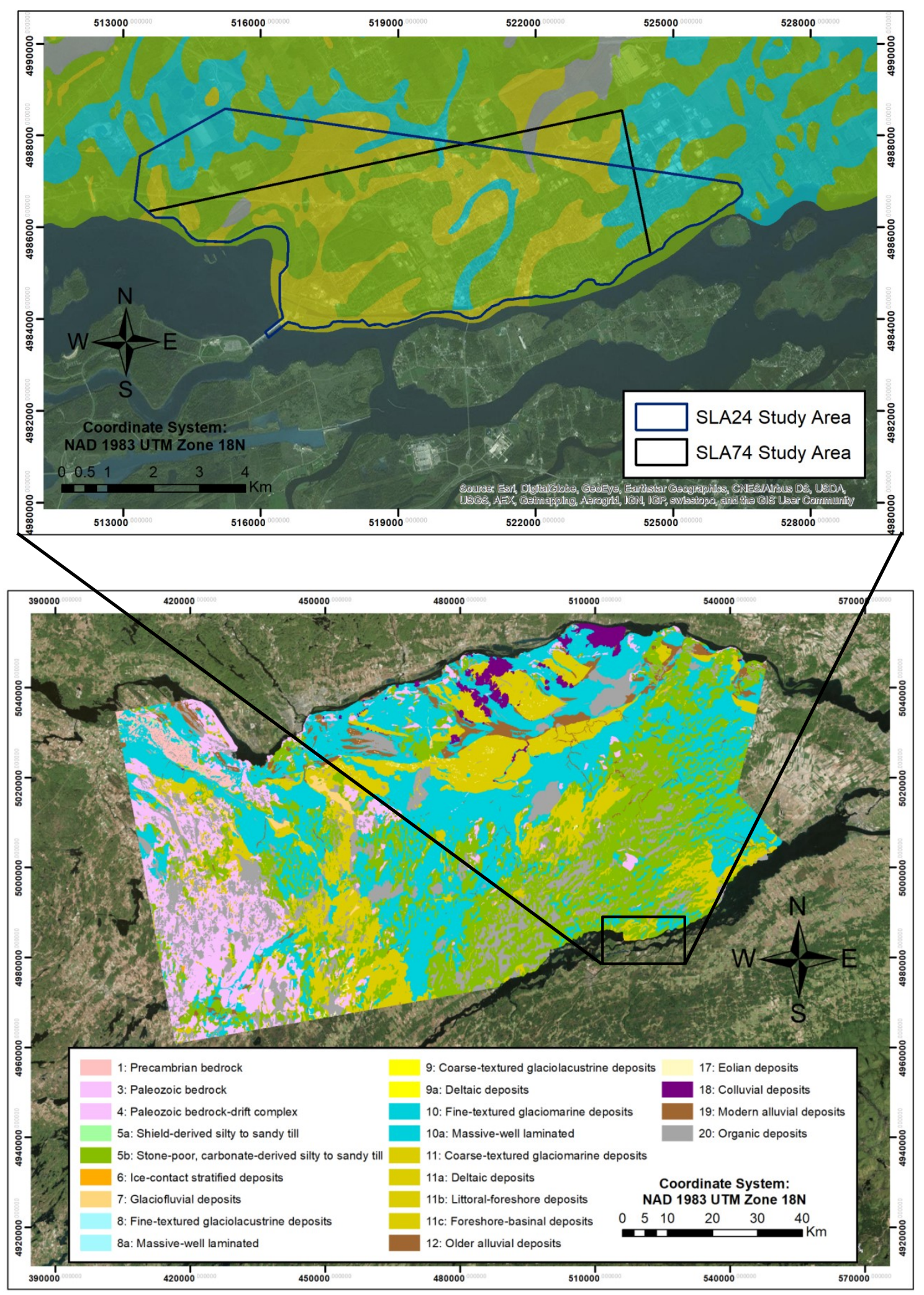

Figure 13: Surficial geology of (top) Cornwall, Ontario and (bottom) subset of Eastern Ontario. 


\section{Chapter 5: Methodology}

In this chapter the processing sequences applied to the RADARSAT-2 and Sentinel-1 datasets are described. These processing sequences are similar, with the only differences being those required as a result of the TOPSAR acquisition mode of the IW, Sentinel-1 dataset.

Except where otherwise noted, all processing has been performed with the GAMMA SAR and Interferometry Software Package (henceforth referred to as GAMMA) (Gamma Remote Sensing 2015b). The Sentinel Application Platform (SNAP) (ESA 2017e) is used in Section 7.2 in support of the Sentinel-1 analysis of the secondary study site.

\subsection{RADARSAT-2}

The RADARSAT-2 dataset consists of acquisitions taken in ascending (SLA74) and descending (SLA24) orbits. Each was processed separately with an identical processing sequence, first the SLA74 dataset, followed by the SLA24 dataset. The RADARSAT-2 processing sequence consists of two iterations, which are described in detail below.

\subsubsection{Iteration 1}

Iteration 1 (Figure 14) consists of three stages. In the first, the SLC images were coregistered to a common geometrical master. In the second, the area of interest was selected, multilook processing was performed, and the geocoding transformation between RDC and UTM coordinates was established. In the final stage, a suite of 
filtered, unwrapped, differential interferograms was generated and used as input to iteration 2. 


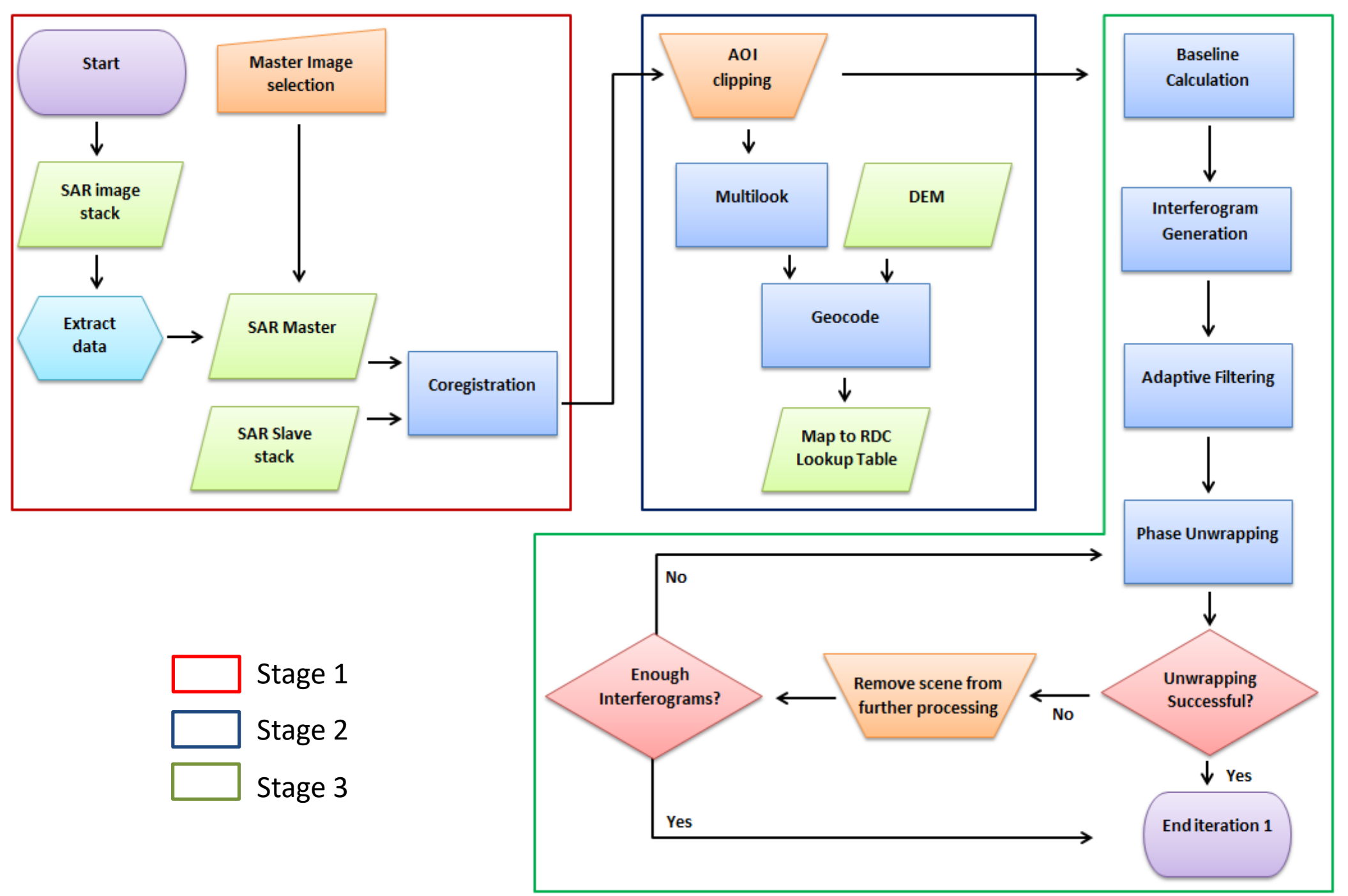

Figure 14: Flowchart of iteration 1, which consists of three stages. In stage 1 (red), the SLC images are extracted, a geometrical master selected, and the slave images coregistered to this master. In stage $\mathbf{2}$ (green), the study area is clipped from each of the coregistered SLC's, multi-looking is performed, and the geocoding transformation established. In stage 3 , baselines are calculated and a corresponding suite of filtered, unwrapped, differential interferograms generated. 


\section{Stage 1: Coregistration}

Each SLC image was first extracted into the internal format used by GAMMA and assigned an image ID (Tables 3 and 4). One of these SLC images was selected as the geometrical master, and the rest coregistered with respect to this image. A coregistration accuracy limit of 0.2 pixels or better was implemented, i.e. if a SLC image could not be coregistered to within $20 \%$ of a pixel then it would be discarded from the analysis. Coregistration was done in five steps:

1. Prior to resampling, the size and position of the correlation patches to be used in step 4 are determined from the master and slave image geometries.

2. An estimate of the constant offset between the centers of the two images was made. This offset estimate was calculated from the orbital state vector information associated with the geometrical master and slave SLC's.

3. The constant offset was refined using GAMMA's two dimensional crosscorrelation algorithm. An input parameter at this step is the center position for a region containing topographic features, which is then used to perform the crosscorrelation of image intensity. Since Cornwall is fairly flat, the Alcoa East Plant in Massena, NY, USA was used instead (Figure 15). This feature is located somewhat centrally in both SLA24 and SLA74 datasets, has a high intensity in the SAR images, and is adjacent to the St. Lawrence River, which provides significant intensity contrast.

4. The offsets between images were calculated for each of the offset positions established in step 1 by using GAMMA's cross-correlation of intensity algorithm. 
This procedure takes into account the image offset calculated in step 2 and refined in step 3. From these offsets, range and azimuth resampling polynomials, which describe the resampling of the slave image, were then calculated.

5. The slave image was iteratively resampled to the geometry of the geometrical master. The range and azimuth resampling polynomials established in step 3 were used to resample the slave image, and subsequently, the offsets for each offset position were re-calculated, range and azimuth resampling polynomials updated, and the slave image further resampled. As a measure of the quality of coregistration, the standard deviation of residual offsets (in range and azimuth) with respect to the updated range and azimuth resampling polynomials were calculated.

For coregistration to be deemed successful, i.e. to know that the coregistration accuracy limit of 0.2 pixels or better was achieved, the standard deviation of residual offsets in both the range and azimuth direction must be below 0.1 . The coregistration procedure was repeated numerous times with different geometrical masters and crosscorrelation of image intensity center locations, and each time the range and azimuth standard deviations examined. The most ideal geometrical master images were determined to be 2016-04-08 and 2016-05-08 for the SLA24 and SLA74 datasets, respectively. Tables 3 and 4 list the standard deviation of residual offsets. One SLA24 image (2016-02-20) and two SLA74 images (2015-03-03 and 2016-02-26) could not be satisfactorily coregistered. All of these images were acquired in the winter months and, as seen in Table 1, had snow cover. 
Table 3: SLA24 images, image ID's, and the standard deviation of residual offsets (in range and azimuth) of the coregistered images

\begin{tabular}{|c|c|c|c|}
\hline Acquisition Date & Image ID & Range std. dev. (pixel) & Azimuth std. dev. (pixel) \\
\hline $2015-03-21$ & 1 & 0.0283 & 0.0599 \\
\hline 2015-04-14 & 2 & 0.0285 & 0.0742 \\
\hline 2015-05-08 & 3 & 0.0382 & 0.0835 \\
\hline 2015-06-01 & 4 & 0.0694 & 0.0892 \\
\hline $2015-06-25$ & 5 & 0.0726 & 0.0854 \\
\hline 2015-08-12 & 6 & 0.0932 & 0.0630 \\
\hline 2015-09-05 & 7 & 0.0986 & 0.0658 \\
\hline $2015-10-23$ & 8 & 0.0282 & 0.0559 \\
\hline 2015-11-16 & 9 & 0.0259 & 0.0619 \\
\hline $2016-02-20$ & 10 & 0.0511 & 0.1294 \\
\hline 2016-03-15 & 11 & 0.0211 & 0.0570 \\
\hline 2016-04-08 & 12 & Geometrical Master & Geometrical Master \\
\hline 2016-05-02 & 13 & 0.0272 & 0.0555 \\
\hline $2016-05-26$ & 14 & 0.0641 & 0.0711 \\
\hline 2016-06-19 & 15 & 0.0854 & 0.0848 \\
\hline 2016-07-13 & 16 & 0.0961 & 0.0735 \\
\hline 2016-08-06 & 17 & 0.0761 & 0.0608 \\
\hline $2016-08-30$ & 18 & 0.0615 & 0.0449 \\
\hline $2016-09-23$ & 19 & 0.0697 & 0.0780 \\
\hline
\end{tabular}


Table 4: SLA74 images, image ID's, and the standard deviation of residual offsets (in range and azimuth) of the coregistered images

\begin{tabular}{|l|l|l|l|}
\hline Acquisition Date & Image ID & Range std. dev. (pixel) & Azimuth std. dev. (pixel) \\
\hline $\mathbf{2 0 1 5 - 0 3 - 0 3}$ & 1 & 0.0462 & 0.1585 \\
\hline $\mathbf{2 0 1 5 - 0 3 - 2 7}$ & 2 & 0.0339 & 0.0965 \\
\hline $\mathbf{2 0 1 5 - 0 4 - 2 0}$ & 3 & 0.0352 & 0.0973 \\
\hline $\mathbf{2 0 1 5 - 0 6 - 0 7}$ & 4 & 0.0299 & 0.0749 \\
\hline $\mathbf{2 0 1 5 - 0 7 - 2 5}$ & 5 & 0.0297 & 0.0783 \\
\hline $\mathbf{2 0 1 5 - 0 8 - 1 8}$ & 6 & 0.0277 & 0.0769 \\
\hline $\mathbf{2 0 1 5 - 0 9 - 1 1}$ & 7 & 0.0299 & 0.0715 \\
\hline $\mathbf{2 0 1 6 - 0 2 - 2 6}$ & 8 & 0.0363 & 0.1141 \\
\hline $\mathbf{2 0 1 6 - 0 3 - 2 1}$ & 9 & 0.0256 & 0.0721 \\
\hline $\mathbf{2 0 1 6 - 0 5 - 0 8}$ & 10 & Geometrical Master & Geometrical Master \\
\hline $\mathbf{2 0 1 6 - 0 6 - 0 1}$ & 11 & 0.0224 & 0.0590 \\
\hline $\mathbf{2 0 1 6 - 0 6 - 2 5}$ & 12 & 0.0253 & 0.0689 \\
\hline $\mathbf{2 0 1 6 - 0 7 - 1 9}$ & 13 & 0.0273 & 0.0715 \\
\hline $\mathbf{2 0 1 6 - 0 8 - 1 2}$ & 14 & 0.0256 & 0.0258 \\
\hline $\mathbf{2 0 1 6 - 0 9 - 0 5}$ & 15 & 0.0620 \\
\hline
\end{tabular}




\section{Stage 2: Area of interest selection, multi-looking, and geocoding}

The area of interest was selected and clipped from the coregistered SLC's. These images share the same dimensions, but do not necessarily cover the same extent. Due to minor variations in satellite orbit, the coverage area of each image varies slightly and therefore coregistration to the geometrical master results in no-data bands along the edges of some of the resampled SLC's (Figure 15). The boundary of the area of interest was chosen to:

1. Contain as much of the City of Cornwall as possible. Cornwall is not fully imaged by either dataset. Each of the coregistered images was examined prior to determining the extent of the study area so that no-data bands were not included in the analysis.

2. Maximize the length of linear infrastructure elements.

3. Not extend much further south than the Saint Lawrence River.

4. Not extend into a no-data band on any of the resampled SLC's.

After a suitable area of interest was established, the resampled SLC's were clipped to these dimensions. For SLA24, the area of interest is the $8920 \times 12800$ pixels region beginning at position $(1080,1500)$. For SLA74, the area of interest is the $4300 \times 15000$ pixels region beginning at position $(2000,8000)$. Subsequently, multilook processing was performed on these clipped, resampled SLC's to create a coregistered set of MLI images. 

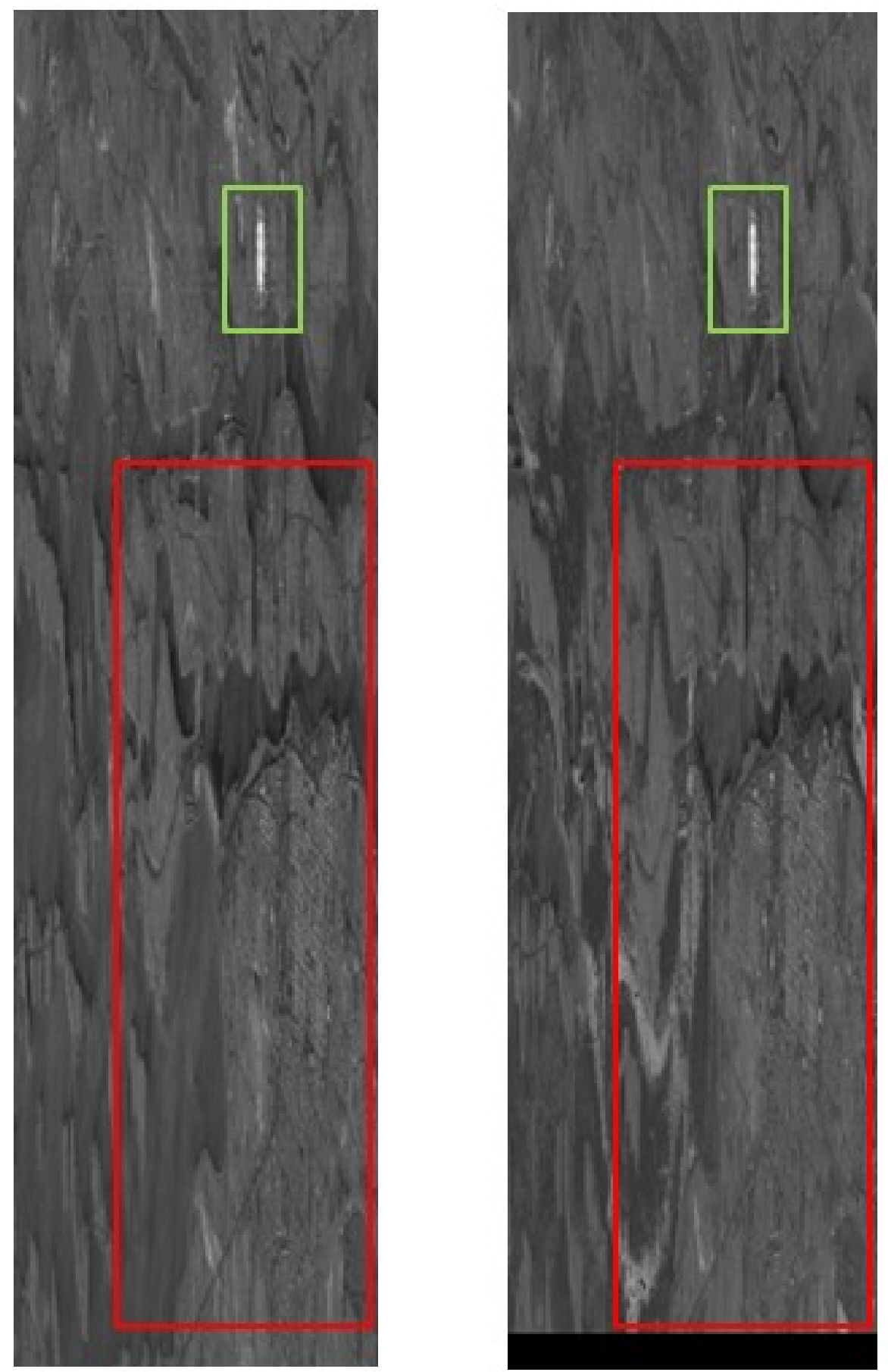

Figure 15: (Left) SLC image of the geometrical master of the SLA74 dataset (2016-05-08) and (right) slave SLC image (2016-02-26) after resampling to geometry of the geometrical master, prior to clipping and multilook processing. The red box is the extent of the area clipped from the SLC's. The green box is the Alcoa East Plant in Massena, NY, USA, used to aid the coregistration. 
A SAR image is significantly easier to interpret if the pixels are approximately square, i.e. if the range and azimuth pixel spacing's are roughly equal. Multilook processing ratios were selected to minimize the reduction in spatial resolution, while also achieving roughly square pixels, i.e. the lowest ratio that resulted in negligible image distortions were used. For the SLA24 dataset a 1:2 (range to azimuth) multilook processing ratio was used, and for the SLA74 dataset a 1:3 ratio was used. After multilook processing, the coregistered $\mathrm{MLI}$ images were averaged to create an image that has significantly reduced speckle compared to a single MLI image. This average MLI image is used for presentation and geocoding purposes (Figure 16).

The transformation between UTM and RDC is required so that: 1) MLI images, interferograms, deformation maps, etc. can be presented in map coordinates; and 2) the DSM can be transformed from UTM to RDC. A DSM in RDC is required in stage 3 (and likewise in iteration 2) to remove the topographic phase from interferograms. Geocoding was performed in four steps:

1. The extent of the study area was extracted from the DSM, a rough transformation between RDC and UTM coordinates established, and a simulated SAR image generated. As seen in Figure 11, the extent of the DSM is significantly larger than the study area to the north. Based off of the dimensions of the MLI, and coordinates of the center, a section of the DSM covering only the area of interest was extracted. The rough transformation between UTM and RDC was then calculated from the orbital state vector information associated with the 
average $\mathrm{MLI}$ image, and the extent of the DSM section. The accuracy of this rough transformation is limited by the accuracy of the orbital state vector information, and therefore refinement was required. From the DSM section and rough UTM to RDC transformation, a simulated SAR image in RDC was calculated and used in steps 2 and 3 to perform the required refinement.

2. The rough transformation was refined by determining a residual constant offset between the simulated SAR and average MLI images. This offset was calculated with GAMMA's two dimensional cross-correlation algorithm for MLI's, applied to the full scene. Similarly to step 3 of the coregistration, a high-intensity feature may be selected to aid the offset estimation. In this step, since the area of interest has already been selected and contains an urban area, manual selection of the offset position was not required, and instead the image center was used.

3. The transformation was further refined by calculating a field of offsets between the simulated SAR and average MLI images over a grid. This was done by subsetting the image into patches of 256x256 pixels and applying GAMMA's two dimensional cross-correlation algorithm to each patch.

4. The refined transformation was applied to the DSM to create a DSM in RDC. The inverse transformation was applied to the average MLI image, to create a geocoded MLI image.

All products (interferograms, coherence maps, displacement maps, etc.) from later processing stages are generated in RDC, and multilook processing was performed with the same ratio used to generate the average $\mathrm{MLI}$ image. Therefore, the inverse UTM to 
RDC transformation established here can be applied to any of product, at any processing stage, to present it in map coordinates. 

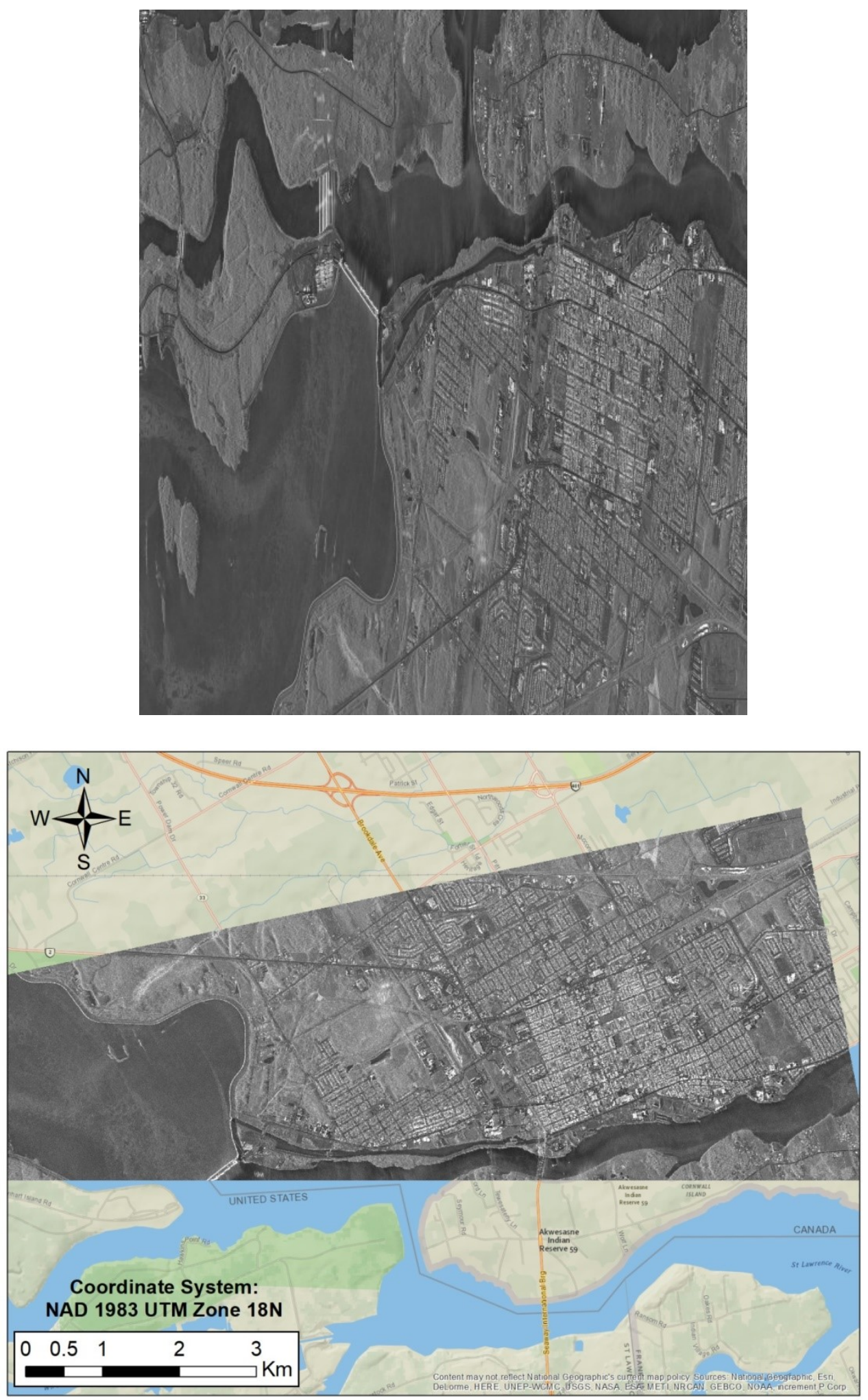

Figure 16: (Top) the SLA74 study area (i.e. the red box in Figure 15) in RDC after clipping and 1:3 multilook processing. (Bottom) the SLA74 study area in UTM coordinates. 


\section{Stage 3: Filtered, unwrapped, differential interferograms}

The inputs to stage 3 are the coregistered set of SLC's clipped to contain the area of interest, and the DSM in RDC generated in stage 2. Prior to calculating differential interferograms, the baseline (i.e. the perpendicular and parallel component of the baseline vector) for each interferometric master and slave pair was calculated (Figure 17). Since the multi-baseline algorithm implemented in iteration 2 uses a multi-master approach, the baseline for every interferometric master and slave pair with a temporal baseline of 365 days or less was calculated. The temporal baseline limit of 365 days was implemented to reduce computational requirements and minimize the number of interferograms with temporal decorrelation. Optionally, a maximum baseline could have been specified to prevent interferograms with large baseline components from being calculated. However, since the RADARSAT-2 platform has good orbital control, it was assumed that the magnitude of the baselines would be small enough such that baseline decorrelation would not occur. Furthermore, the topography in the study area is minimal and a high quality DSM was used, and as such the topographic phase should be easily quantifiable and removed, even on interferograms with relatively large baseline components. For the SLA24 dataset, 111 baselines were calculated, and for the SLA74 dataset, 72 baselines were calculated. 
Perpendicular baseline (m) vs. temporal baseline (days) for SLA74 dataset

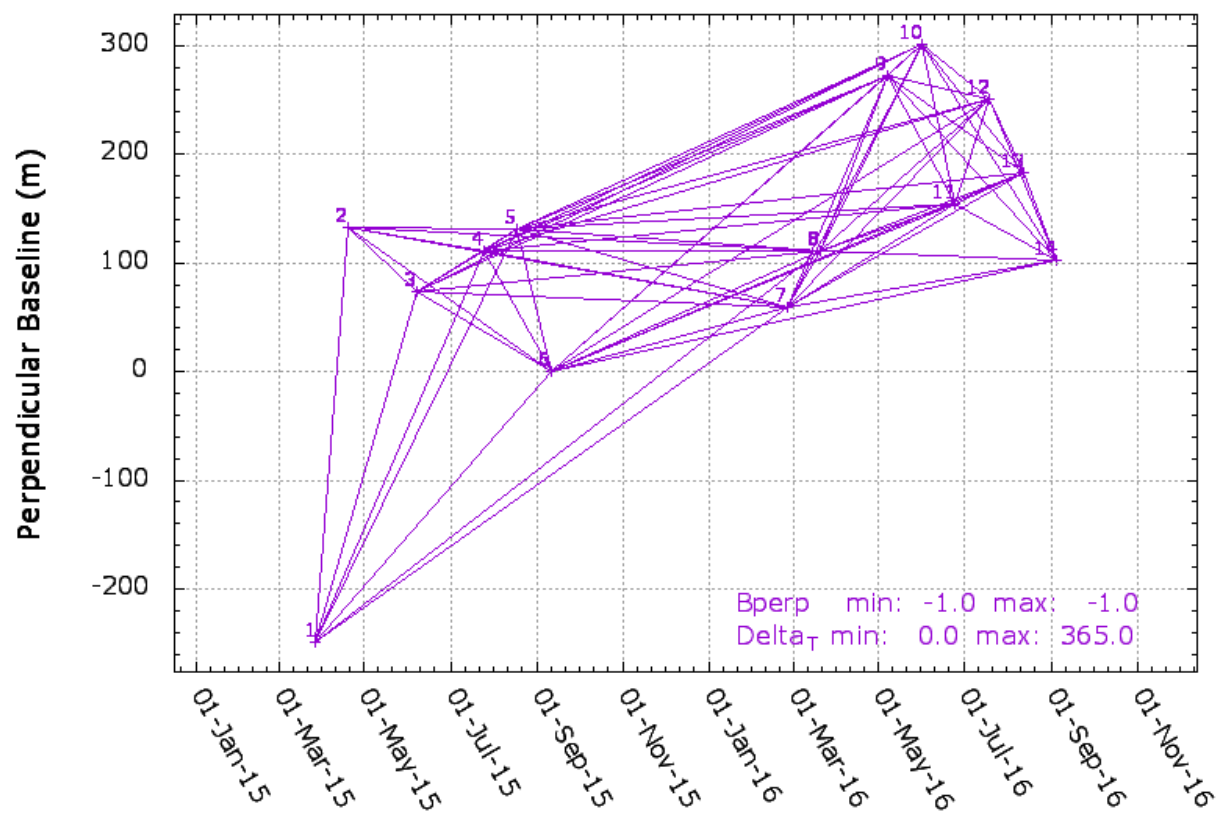

Perpendicular baseline (m) vs. temporal baseline (days) for SLA24 dataset

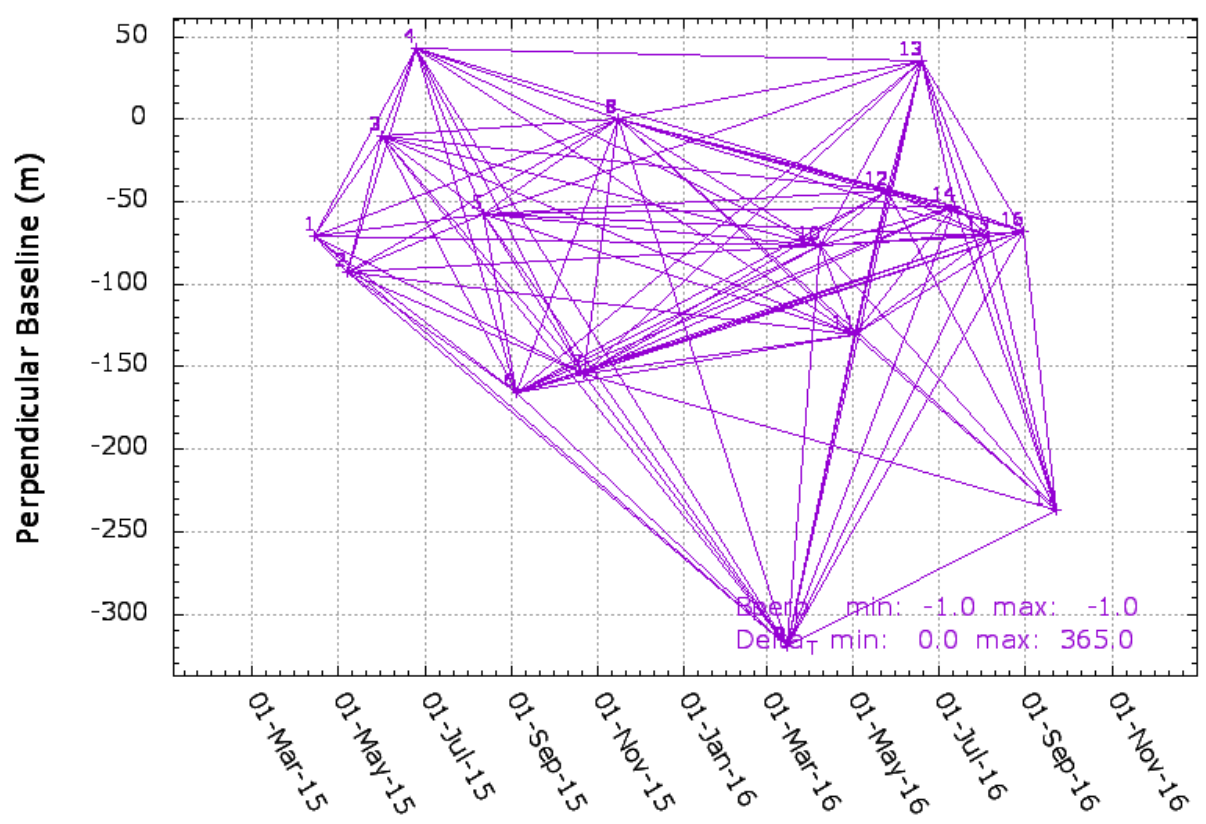

Figure 17: Perpendicular baseline (m) vs. temporal baseline (days) for the SLA74 (top) and SLA24 (bottom) datasets. 
For each master-slave pair, the associated differential interferogram was then calculated. As described in Section 3.2., first the complex interferogram was calculated, and subsequently a simulation of the topographic phase calculated from the DSM in $\mathrm{RDC}$ and the baseline information was subtracted from the interferogram. Interferometric coherence maps were also generated for each differential interferogram.

The interferograms were filtered using GAMMA's adaptive spectral filtering algorithm to reduce phase noise (which is based off Goldstein et al. 1998), and then unwrapped. Phase unwrapping was performed with GAMMA's implementation of the MCF algorithm. For both the SLA24 and SLA74 datasets, a coherence limit of 0.5 was implemented in the phase unwrapping. The coherence limit was determined by manually examining the Saint Lawrence River in each of the coherence maps associated with the differential interferograms. The expected coherence for pixels over the Saint Lawrence River (i.e. a water body) is (close to) zero. However, due to phase noise, many pixels with coherence greater than zero were found. The highest coherence values for pixels associated to the Saint Lawrence River were between 0.40 and 0.45 for all interferograms. Therefore a coherence limit of 0.50 prevents the phase unwrapping of noisy pixels, which contribute to phase unwrapping errors.

The Cornwall Community Hospital (Figure 20) was selected as the reference region because: 1 ) it is located somewhat centrally within both study areas; 2 ) it is a highly coherent feature throughout the interferogram suite; and 3) it can be assumed to 
be a stable structure experiencing little to no deformation. This assumption is based off the fact that hospitals are generally constructed with pile foundations, and that the Cornwall Community hospital is built on a deposit of sandy and silty compacted diamicton which would be expected to experience little to no compaction or consolidation.

Each of the filtered, unwrapped, differential interferograms was then manually examined; those with prominent phase unwrapping errors or very low coherence were removed from further processing. Since this interferogram suite is only used (in iteration 2) for the baseline refinement process and not in the multi-baseline analysis, interferograms with small phase unwrapping errors were accepted for further analysis. No quantitative criteria to distinguish between prominent vs. small phase unwrapping errors or acceptable coherence levels were implemented. Figure 18 shows examples of interferograms with: a) prominent phase unwrapping errors; b) small but acceptable phase unwrapping errors; c) no phase unwrapping errors but unacceptably low coherence; and d) no phase unwrapping errors and high coherence. For the SLA24 dataset, 91 out of 111 interferograms were accepted. Every interferogram made with scene 2016-03-15 was rejected, and so this image was removed from further processing. For the SLA74 dataset, 63 out of 72 interferograms were accepted, and no additional scenes were removed. 


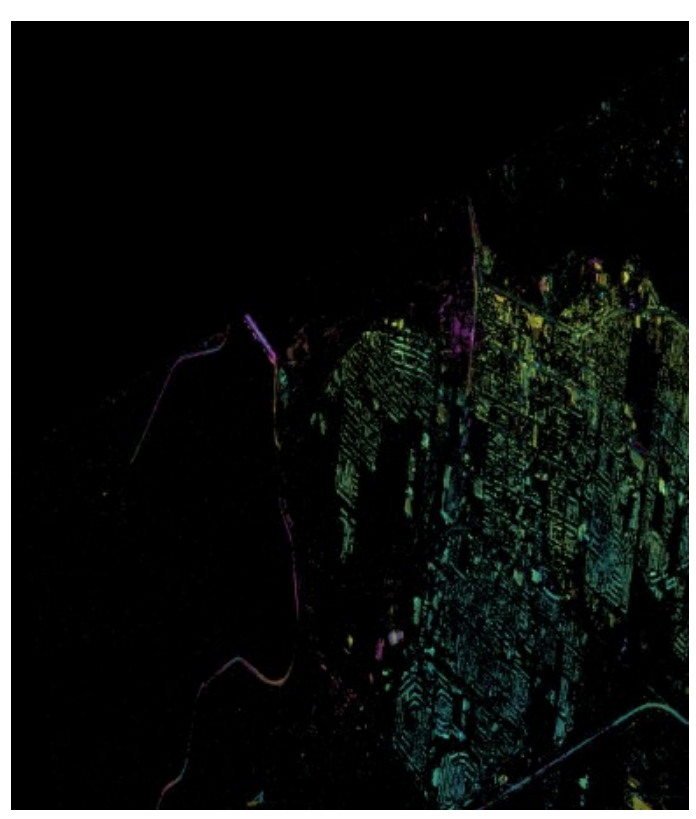

a) SLA74: $20150327-20150420$

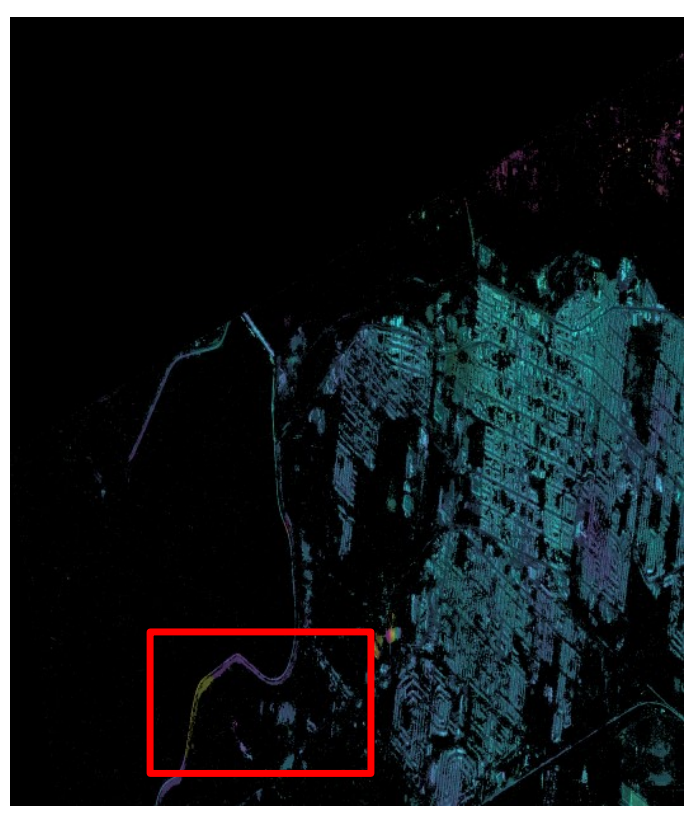

b) SLA74: $2015725-20160508$

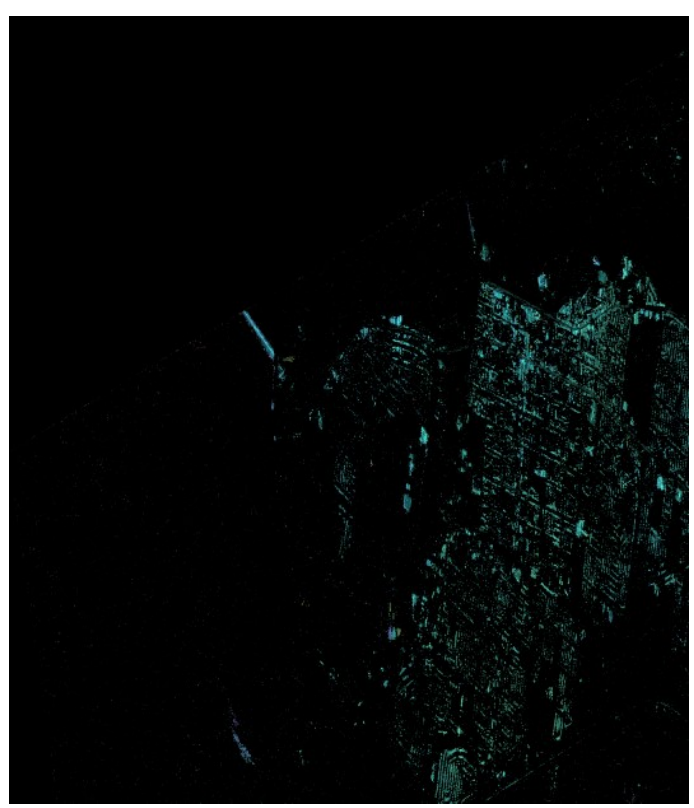

c) SLA74: $20150420-20160226$

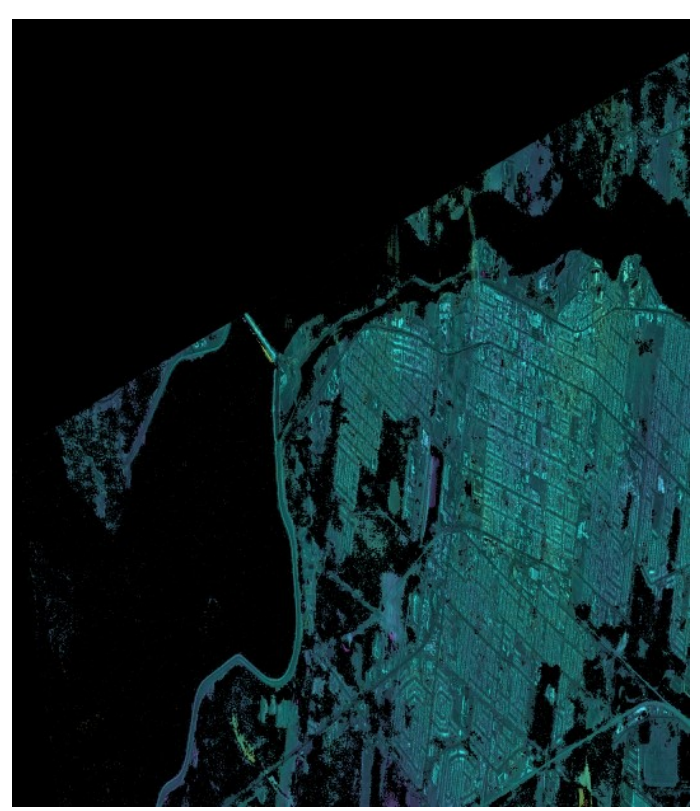

d) SLA74: 20150725-20150818

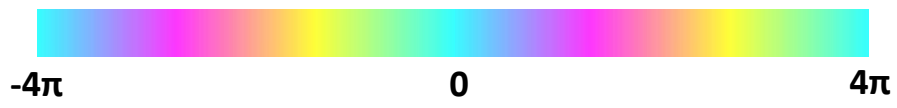

Figure 18: a) An interferogram with good coherence, but prominent unwrapping errors. b) An interferogram with good coherence, but some small unwrapping errors (red box). c) An interferogram with no unwrapping errors, but poor coherence due to snow cover on 2016-02-26. d) An ideal interferogram with high coherence and no unwrapping errors. The interferograms are presented at $4 \pi$ per colour cycle. 


\subsubsection{Iteration 2}

Iteration 2 consists of two stages (Figure 19). In stage 1, baseline refinement was performed on the interferogram suite established in iteration 1, and subsequently a new suite of baseline-refined, filtered, unwrapped, differential interferograms was generated. These interferograms were examined for atmospheric effects and, in stage 2, deformation was calculated by applying GAMMA's multi-baseline algorithm to suitable interferograms. The resultant deformation maps were then geocoded and exported to ArcMap. 


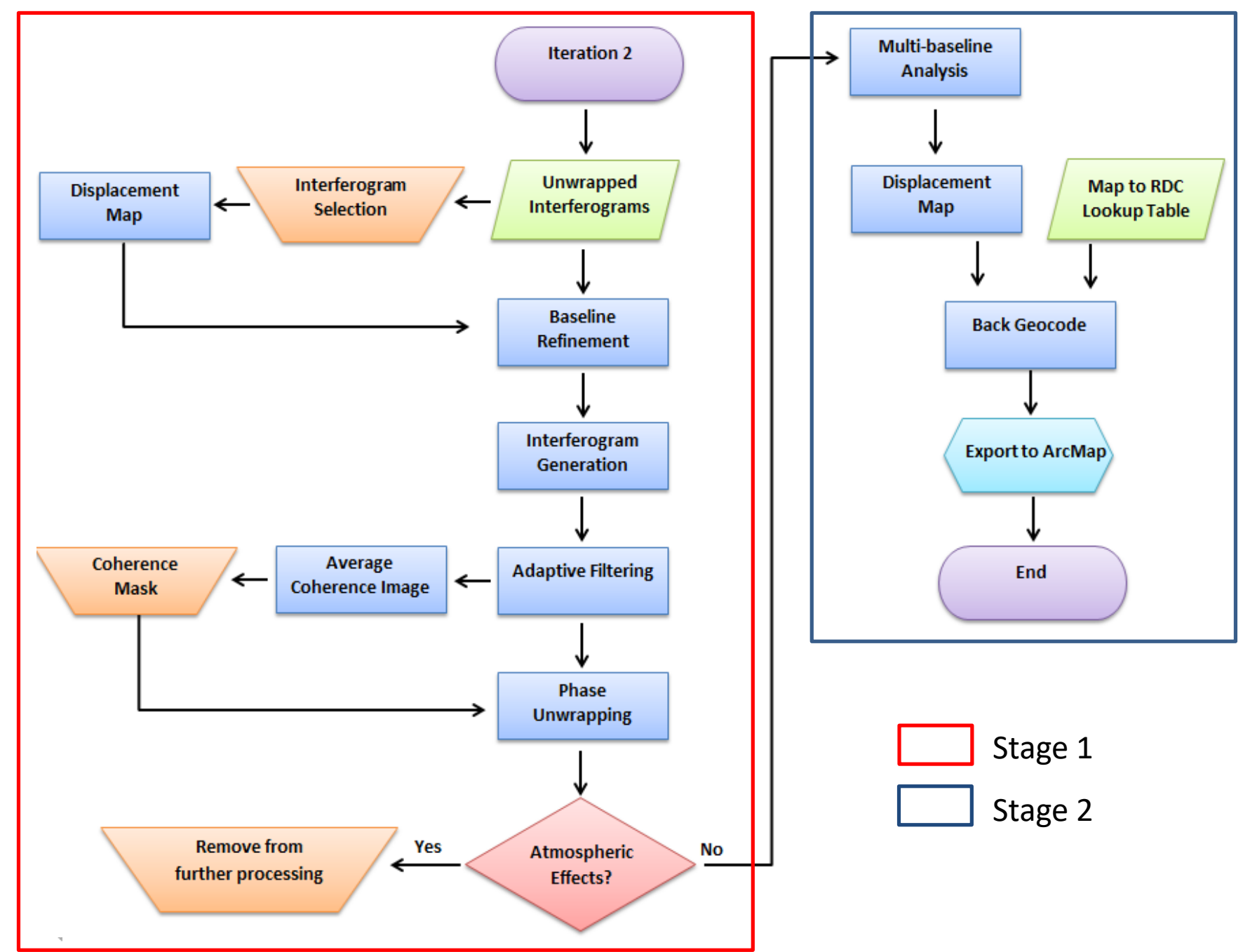

Figure 19: Flowchart of iteration 2, which consists of two stages. In stage 1 (red), baseline refinement is performed on the interferograms selected from iteration 1 with the aid of a displacement mask, and subsequently a new suite of filtered, unwrapped, differential interferograms is generated. In stage 2 , interferograms with minimal atmospheric effects are used to perform the multi-baseline analysis, and displacement maps are created, geocoded, and exported to ArcMap. 


\section{Stage 1: Baseline refinement and interferogram generation}

The input to stage 1 is the suite of filtered, unwrapped, differential interferograms established in iteration 1. For each of these interferograms, GAMMA's baseline refinement procedure was applied. Generally, baseline refinement should be performed by only considering stable regions; pixels representing rapid deformations should be masked out prior to refinement. In this project (and as would be the case in general when monitoring geohazards), there was no a priori knowledge regarding the location and time of specific ground deformations. A single filtered, unwrapped, differential interferogram was selected, and from this interferogram a deformation map created. From this deformation map, a deformation mask was created by applying a threshold to the map; pixels with deformation greater than the specified value were assigned a pixel value of zero, and pixels with deformation less than the threshold were assigned a value of 1 . Baseline refinement was only performed on pixels that had a value of 1 in the displacement mask. The threshold value was determined qualitatively such that the displacement mask contained the majority of the same pixels as the displacement map, with only small noisy features (street lights, vents on building tops, transmission towers) being masked.

The interferogram used for creating the displacement mask was selected on the basis of the following criteria: 1) very high coherence; 2) minimal phase unwrapping errors; and 3) relatively small residual baseline effects. Criterion 1) is required so that the number of pixels in the displacement mask is maximized; if a low coherence interferogram was selected, then the number of pixels in the mask would be small. 
Criteria 2) and 3) are required since phase unwrapping errors and residual baseline effects cannot be distinguished from deformation in a single interferogram. The interferograms were selected based off these criteria in a qualitative, semi-empirical way based off of visual examination of the interferogram suite. For the SLA24 dataset, interferogram 2015-09-05 to 2015-10-23 with a deformation threshold of $\pm 2 \mathrm{~mm}$ was selected. For the SLA74 dataset, interferogram 2015-08-18 to 2015-09-11 with a deformation threshold of $\pm 5 \mathrm{~mm}$ was selected.

Differential interferograms (and associated coherence maps) were then regenerated, this time using the refined baselines to remove the topographic phase, and subsequently filtered as in iteration 1 . The interferograms were then unwrapped. In iteration 1, a coherence limit of 0.5 was implemented in the phase unwrapping. Since the coherence of each interferogram varies, however, the amount and location of unwrapped pixels in those unwrapped interferograms also varies. In iteration 2, the unwrapped interferograms are input into the multi-baseline analysis and therefore should each contain the same unwrapped pixels. To achieve this consistency, a coherence-based phase unwrapping mask was created to force the phase unwrapping to be performed on a selected set of pixels.

To make the mask, the coherence maps associated with each of the newly generated differential interferograms were averaged. A threshold was applied to this average coherence map such that pixels with coherence levels less than the threshold were set to zero. Using the GNU Image Manipulation Program (GIMP) cross-platform 
image editor, additional noisy pixels were then manually masked out. The coherence mask used in the phase unwrapping has a significant impact on the amount and severity of phase unwrapping errors, and therefore also on the error level of the final deformation map. For the SLA74 dataset, coherence masks were created using coherence threshold values of $0.45,0.50$, and 0.55 and then manually edited to remove noisy pixels. The SLA74 dataset was unwrapped twice which each of these masks: first with a filled triangular mesh (as in iteration 1) followed by Delaunay triangulation (Gamma Remote Sensing 2015b), totalling six phase unwrapping attempts. All interferograms were then visually examined for significant phase unwrapping errors, and the number of acceptable interferograms for each attempt tallied (Table 5). For the SLA74 dataset, the phase unwrapping mask created with a 0.50 coherence limit and unwrapped with Delaunay triangulation produced the most acceptable interferograms (50 out of 63). The phase unwrapping mask is shown in Figure 20. These same parameters were then used to create an analogous mask for the SLA24 dataset and to perform the phase unwrapping. 
Table 5: Amount of successfully unwrapped interferograms for the SLA74 dataset using coherence masks with various thresholds $(0.45,0.50$, and $\mathbf{0 . 5 5})$ and minimum cost flow triangulation methods (filled triangular mesh or Delaunay triangulation).

\begin{tabular}{|l|l|l|}
\hline Coherence Limit & $\begin{array}{l}\text { Filled Triangular } \\
\text { Mesh }\end{array}$ & $\begin{array}{l}\text { Delaunay } \\
\text { Triangulation }\end{array}$ \\
\hline $\mathbf{0 . 4 5}$ & 43 & 47 \\
\hline $\mathbf{0 . 5 0}$ & 44 & 50 \\
\hline $\mathbf{0 . 5 5}$ & 46 & 46 \\
\hline
\end{tabular}




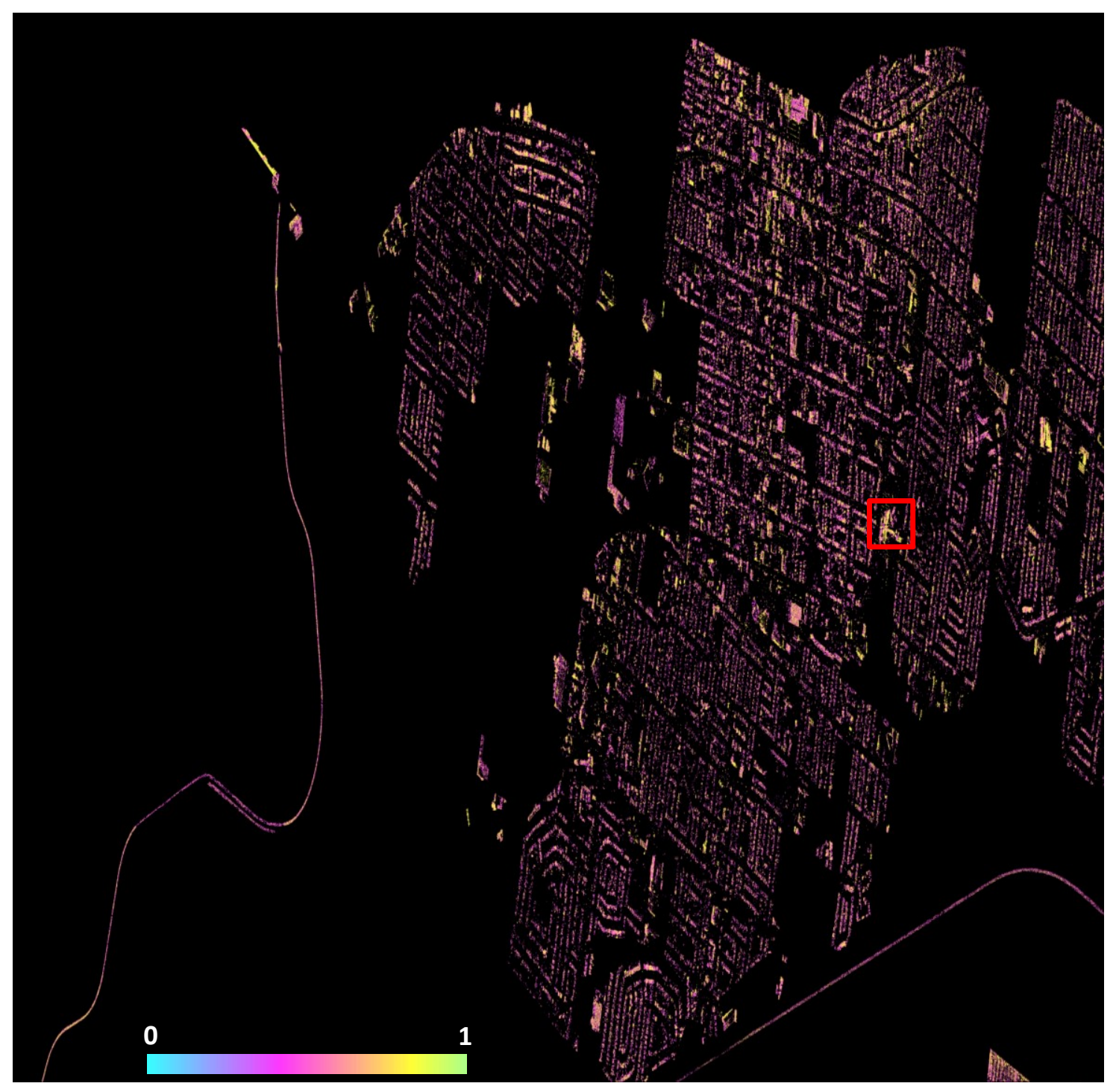

Figure 20: The SLA74 unwrapping coherence mask. Colours correspond to the average coherence value of the selected interferograms. The Cornwall Community Hospital is highlighted by the red box. 
The final step prior to the establishment of the time series of deformation was to re-examine each of the successfully unwrapped interferograms, removing those with significant atmospheric effects from further processing. Both atmospheric effects and ground deformation present themselves in interferograms as regions of anomalously high interferometric phase differences, making distinguishing between these contributions difficult. This difficulty can be partially overcome by recognizing two differences between them. First, the location of atmospheric path delay is uncorrelated from one interferogram to the next, whereas ground deformation will be restricted to a consistent, relatively well defined region in most or all interferograms. Additionally, for regions not experiencing very rapid deformation (such as Cornwall), the magnitude of the interferometric phase difference resulting from atmospheric path delay is often significantly greater than that from ground deformation. Therefore, an interferogram without atmospheric effects will be very close to cyan in colour (since cyan represents zero phase difference in the GAMMA colour scheme, and only small deformations are expected), whereas atmosphere will correspond to magenta and yellow shades. Figure 21 shows an example of an interferogram with significant atmospheric effects, and an ideal interferogram where only minor atmospheric effects are identified. 

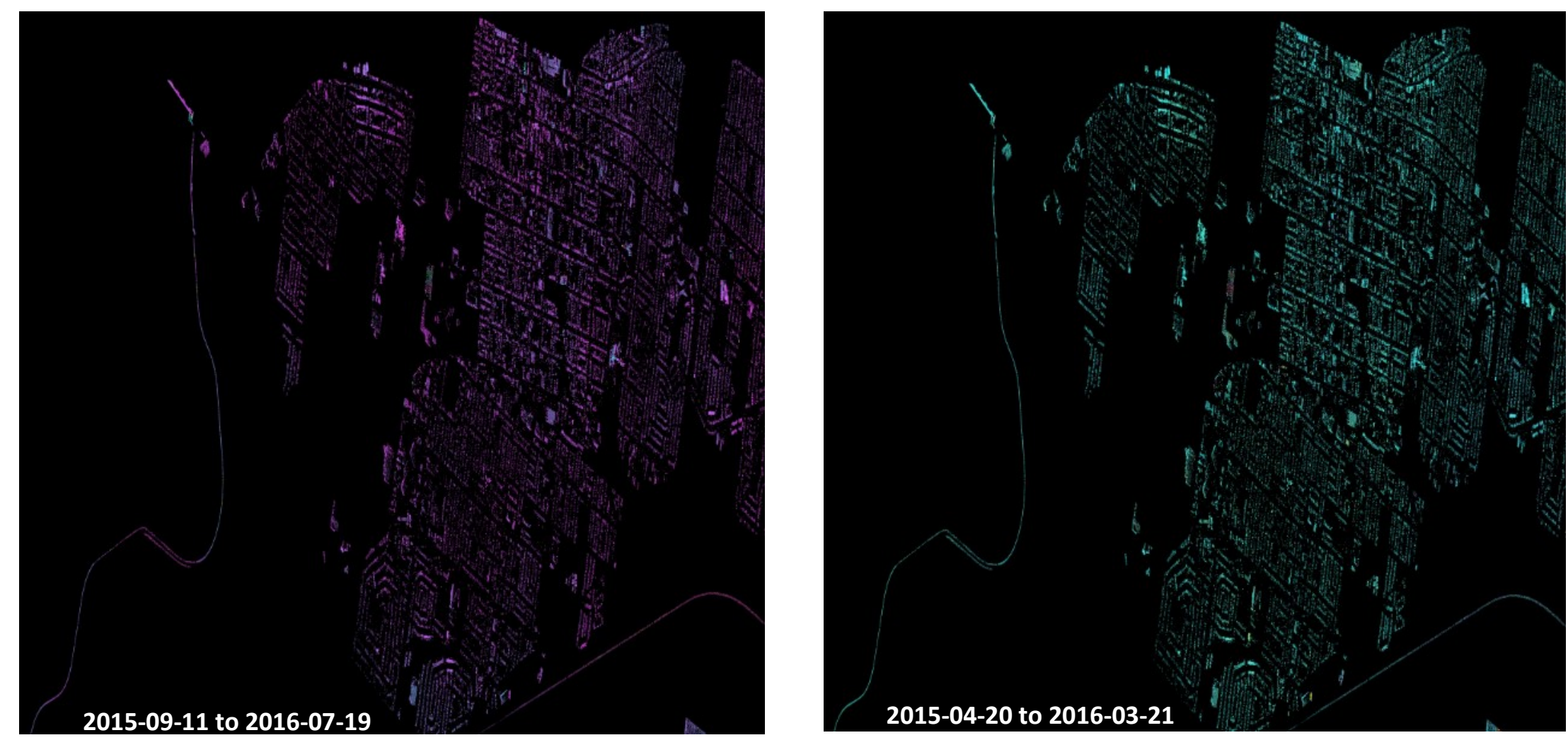

Figure 21: (Left) Example of an interferogram with considerable atmospheric effects. The magenta colour prominent in this interferogram corresponds to an interferometric phase difference of approximately $\pi$, which is too large to represent deformation. (Right) Example of an ideal interferogram with only minor atmospheric effects. The interferogram is predominantly cyan, consistent with small deformations. The interferograms are presented at $4 \pi$ per colour cycle. 
The above selection process was performed qualitatively. Most interferograms show atmospheric effects to some degree, and so whether or not a particular interferogram was accepted or rejected in this step was dependent on the magnitude and location of the atmospheric effects as well as the time interval of that interferogram. A requirement of the multi-baseline algorithm is that the interferogram suite has no temporal gaps i.e. the baseline-time plot remains continuous. Therefore no consistent criteria regarding an acceptable level of atmospheric effects could be implemented. For time intervals containing many interferograms (e.g. the summer months, since many acquisitions were acquired in the summer) a relatively high number of interferograms can be rejected compared to time intervals containing fewer interferograms (e.g. winter, since the datasets contain only a few winter images).

The process of examining the interferogram suite and rejecting interferograms was repeated several times until what was determined to be the best possible selection was established (Figure 22). For the SLA24 dataset, 42 interferograms were accepted; for the SLA74 dataset, 30 interferograms were accepted. One SLA74 scene, 2015-03-27, had atmospheric effects in all interferograms and so was removed from further processing. 
Perpendicular baseline (m) vs. temporal baseline (days) for the SLA74 dataset

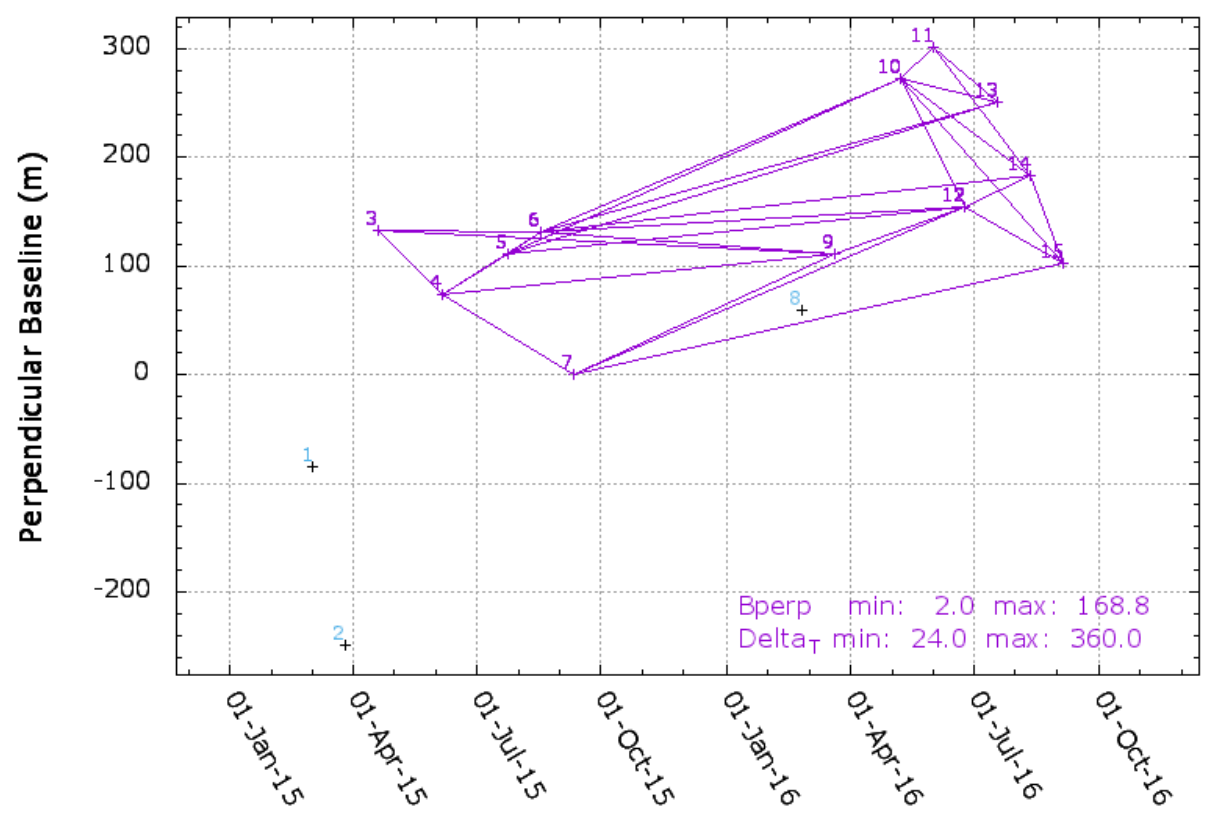

Perpendicular baseline $(\mathrm{m})$ vs. temporal baseline (days) for the SLA24 dataset

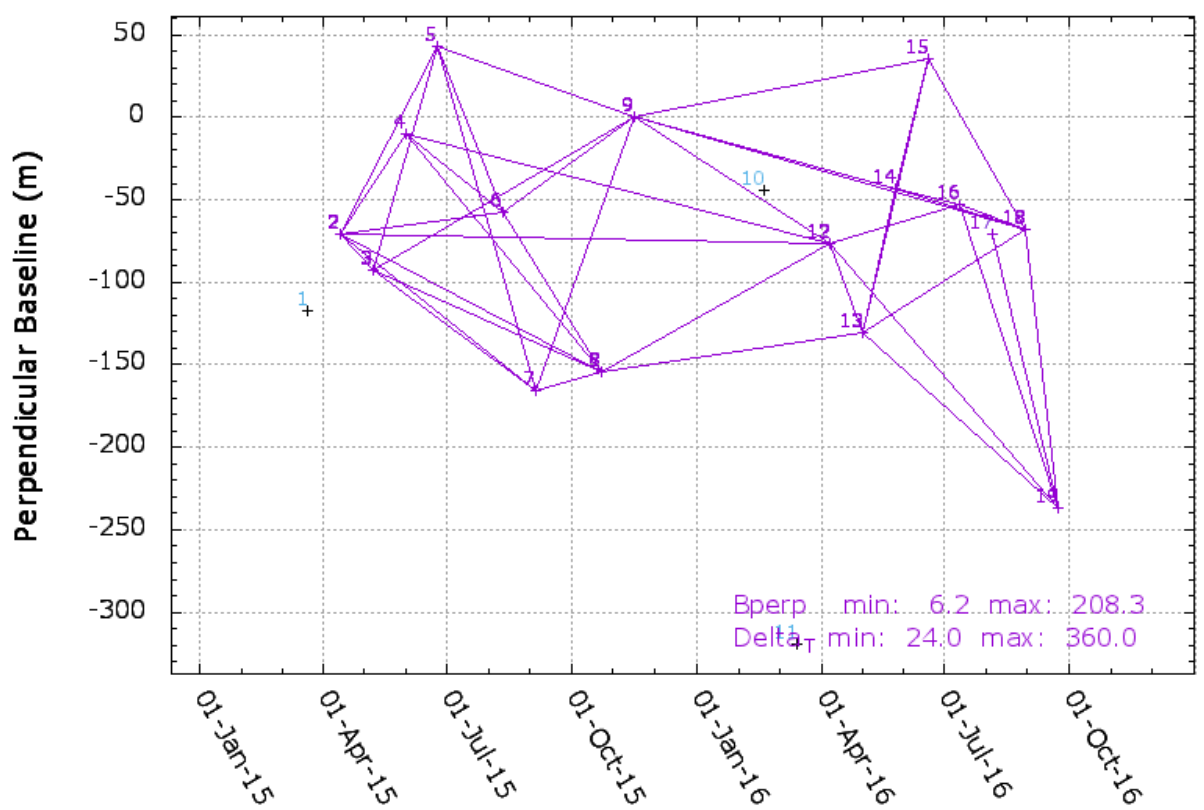

Figure 22: Perpendicular baseline $(\mathrm{m})$ vs. temporal baseline (days) for the SLA74 (top) and SLA24 (bottom) datasets for the interferograms selected for multi-baseline analysis. 


\section{Stage 2: Multi-baseline analysis}

The interferograms selected at the end of stage 1 were then input into GAMMA's multi-baseline analysis algorithm, resulting in a new suite of interferograms. Each interferogram represents the cumulative deformation occurring between each of the images in the dataset, i.e. for the SLA24 dataset, 16 interferograms were produced since 16 images remained in the analysis, and similarly 12 interferograms were produced for the SLA74 dataset. For each of the unwrapped time-series interferograms a vertical displacement map was calculated, and subsequently transformed into UTM coordinates by applying the inverse UTM to RDC transformation established in iteration 1. The deformation map was then exported as a tiff image and georeferenced in ArcMap. Since the displacement maps were geocoded in GAMMA, georeferencing in ArcMap was a straightforward process of defining the projection and inputting the coordinates of the northwest corner of the deformation map. Deformation maps are presented in Section 6.1. 


\subsection{Sentinel-1}

The processing sequence for the Sentinel-1 dataset is similar to that applied to the RADARSAT-2 datasets. The differences between the two are the result of the TOPSAR acquisition mode of the Sentinel-1 platform.

\subsubsection{Iteration 1}

As for the RADARSAT-2 data (Section 5.1.1.), iteration 1 consists of three stages. In the first stage, the SLC images were extracted, concatenated, clipped to a common extent and number of bursts, and finally coregistered to a common geometrical master. In the second stage, the area of interest was selected, multilook processing was performed, and the geocoding transformation between Range-Doppler and UTM coordinates was established. In the final stage, a suite of filtered, unwrapped, differential interferograms was generated and used as input to iteration 2.

\section{Stage 1: Coregistration}

Each burst SLC image was first extracted into the internal format used by GAMMA. As described in Section 4.5.2., for some dates the area of interest was not fully contained within a single image. For these cases, two images, one acquired a few seconds prior to the other (Table 2), were concatenated to form a significantly larger image containing the full area of interest.

Since the timing of the TOPSAR acquisition mode varies slightly from one pass to the next, the number of bursts within each subswath, and likewise the spatial extent of each subswath, varies significantly throughout the dataset. However, coregistration 
should be performed on images corresponding to approximately the same area.

Therefore, prior to coregistration, each burst SLC image was clipped so that the number of bursts within each subswath, as well as the area covered by each subswath, was consistent throughout the dataset. To do this, each burst SLC image was examined and the maximum region common to all images was identified. Subsequently, for each subswath in each image, the bursts corresponding to this common region were selected. For IW1, 3 bursts were selected, and for IW2, 4 bursts were selected. IW3 falls outside of the area of interest, and as such no bursts were selected (i.e. IW3 was removed from further processing).

Coregistration of burst SLC images is required to be extremely accurate or else large phase discontinuities between adjacent bursts will be present in the interferograms. Coregistration accuracy to at least a thousandth of a pixel in the azimuth direction was therefore required. To achieve the required coregistration accuracy a different coregistration procedure than was applied to the RADARSAT-2 datasets was necessary. The transformation from slave to master geometry was first calculated from orbital state vector information and a DSM in RDC (whereas in contrast, in Section 5.1.1. no DSM was used), and refined by iteratively applying the cross-correlation of intensity technique. Following this, examining phase differences in the burst overlap region further refined the transformation. The main processing steps are described below:

1) A coregistration master image, 2015-09-08, was selected and an MLI image generated. For Sentinel-1 IW mode images a multilook ratio of 5:1 (range to 
azimuth) results in approximately square pixels. A DSM in RDC was generated by geocoding the MLI (i.e. establishing the UTM to RDC transformation) by using a procedure similar to that described in Section 5.1.1., and then applying that transformation to the DSM. An estimate of the transformation from slave to master image geometry was then calculated from orbital state vector information. The DSM in RDC aids this estimate for features experiencing layover.

2) The transformation was refined by applying the cross-correlation algorithms in a similar way to steps 3-5 of the RADARSAT-2 coregistration (Section 5.1.1.). This step was applied iteratively until a coregistration accuracy of at least 0.02 pixels was achieved.

3) The transformation was further refined by examining the phase difference in the burst overlap regions. An interferogram corresponding to each burst overlap region was calculated; since bursts were acquired essentially simultaneously, the interferometric phase difference will be zero if the overlap regions are perfectly coregistered. For each burst overlap, the shift required to minimize the interferometric phase difference was iteratively determined and used to refine the image resampling until a coregistration accuracy of 0.005 pixels or better was achieved. Implementing GAMMA's spectral diversity refinement algorithm was used in this process. 


\section{Stage 2: Area of interest selection, multi-looking, and geocoding}

The area of interest was selected from the stack of coregistered SLC images. As described in Section 4.2., Cornwall, Ontario is the primary study location. The Sentinel-1 acquisitions are significantly larger than Cornwall: the width of Cornwall (approximately $10 \mathrm{~km}$ ) is approximately 25 times smaller than the width of a Sentinel-1 swath (which is approximately $250 \mathrm{~km}$ ). Residual baseline errors appear as range and azimuth phase trends in differential interferograms. For a platform with strict orbital control and high quality state vector information (such as Sentinel-1), it can be assumed that in most cases the baseline, and its error, will be small. The corresponding erroneous phase trends then often have large wavelengths of similar magnitude to the image dimensions. Therefore, if just Cornwall were to be selected from an IW image, baseline refinement may not be possible since the erroneous phase trend will be difficult to quantify over such a small region. The extent of the area of interest (the $37000 \times 5500$ pixel region starting at pixel position $(4000,1000)$ of the coregistered SLC's) was selected to contain both the cities of Ottawa and Cornwall, but not extend further north or south than the Ottawa and Saint Lawrence rivers, respectively.

After clipping the coregistered SLC images to the common area of interest, MLI images were created using the same 5:1 ratio as was done previously, and an average MLI image calculated. Since these new MLI images do not have the same dimensions as the MLI image (2015-09-08) used to establish the UTM to RDC transformation in step 1 (that image was not clipped), the same geocoding procedure described in Section 5.1.1. was repeated. The UTM to RDC transformation was applied to the DSM, creating a DSM 
in RDC. As in Section 5.1., all subsequent resampling from UTM to RDC (and vice versa) performed in later stages was performed with this new transformation.

\section{Stage 3: Filtered, unwrapped, differential interferograms}

The inputs to stage 3 are the coregistered set of SLC's clipped to contain the area

of interest, and the DEM in RDC generated in stage 2. As in Section 5.1., prior to calculating differential interferograms, the baseline for every interferometric master and slave pair with a time interval of 365 days or less was calculated (Figure 23). For the Sentinel-1 dataset, 276 interferometric master and slave pairs fit this criterion, and for each of these pairs differential interferograms and coherence maps were calculated. 
Perpendicular baseline (m) vs. temporal baseline (days) for the Sentinel-1 dataset

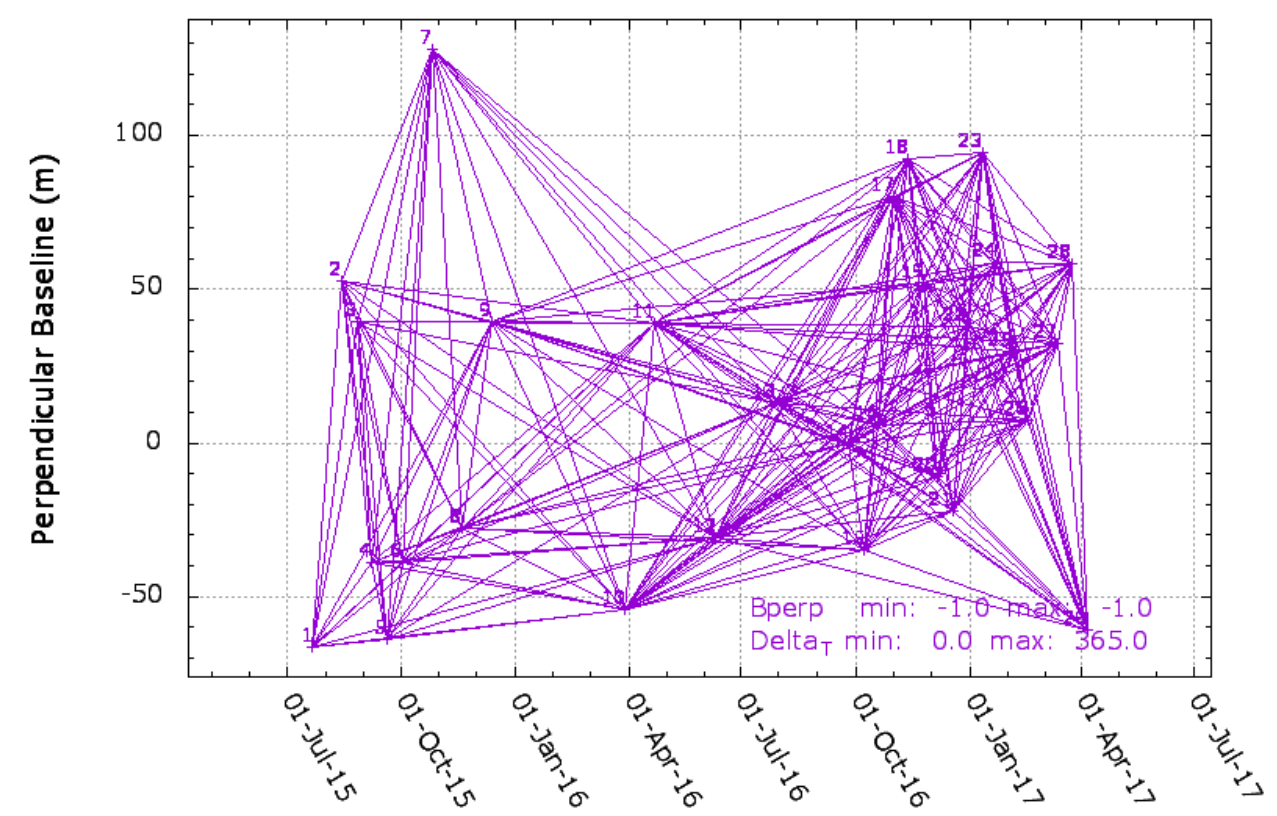

Figure 23: Perpendicular baseline $(\mathrm{m})$ vs. temporal baseline (days) for the Sentinel-1 data 
Subsequently, the differential interferograms were filtered using GAMMA's adaptive spectral filtering algorithm (Goldstein et al. 1998). The purpose of this iteration is to create a suite of unwrapped, differential interferograms that are then used to refine the baselines which were initially estimated from orbital state vectors. To do this refinement, phase trends resulting from baseline errors must be clearly visible across the entire image so that they can be quantified by the GAMMA software. The vast majority of the study area consists of vegetated areas which typically have low coherence, with the cities of Cornwall and Ottawa being relatively small but highly coherent features. Due to the generally low coherence of the study area, the filtering applied at this stage was very strong so that the filtered interferograms had significantly greater fringe visibility than if a standard level of filtering had been applied, as in Section

\subsection{1.}

The heavily filtered interferograms were then unwrapped: the coherence threshold used was 0.30 and the reference point was Parliament Hill in downtown Ottawa. The low coherence limit (compared to the threshold of 0.50 used in Section 5.1.1) was implemented so that phase unwrapping was possible in vegetated areas (i.e. the majority of the scene). Generally a low phase unwrapping coherence threshold of 0.30 would increase the amount of phase unwrapping errors; however, the heavy filtering previously applied reduces phase noise such that this low limit is acceptable. Parliament Hill was selected as the reference region on the basis of its high coherence and central location in Ottawa, with no consideration of its stability. An example of an interferogram with a clear baseline related phase trend is shown in Figure 24. 


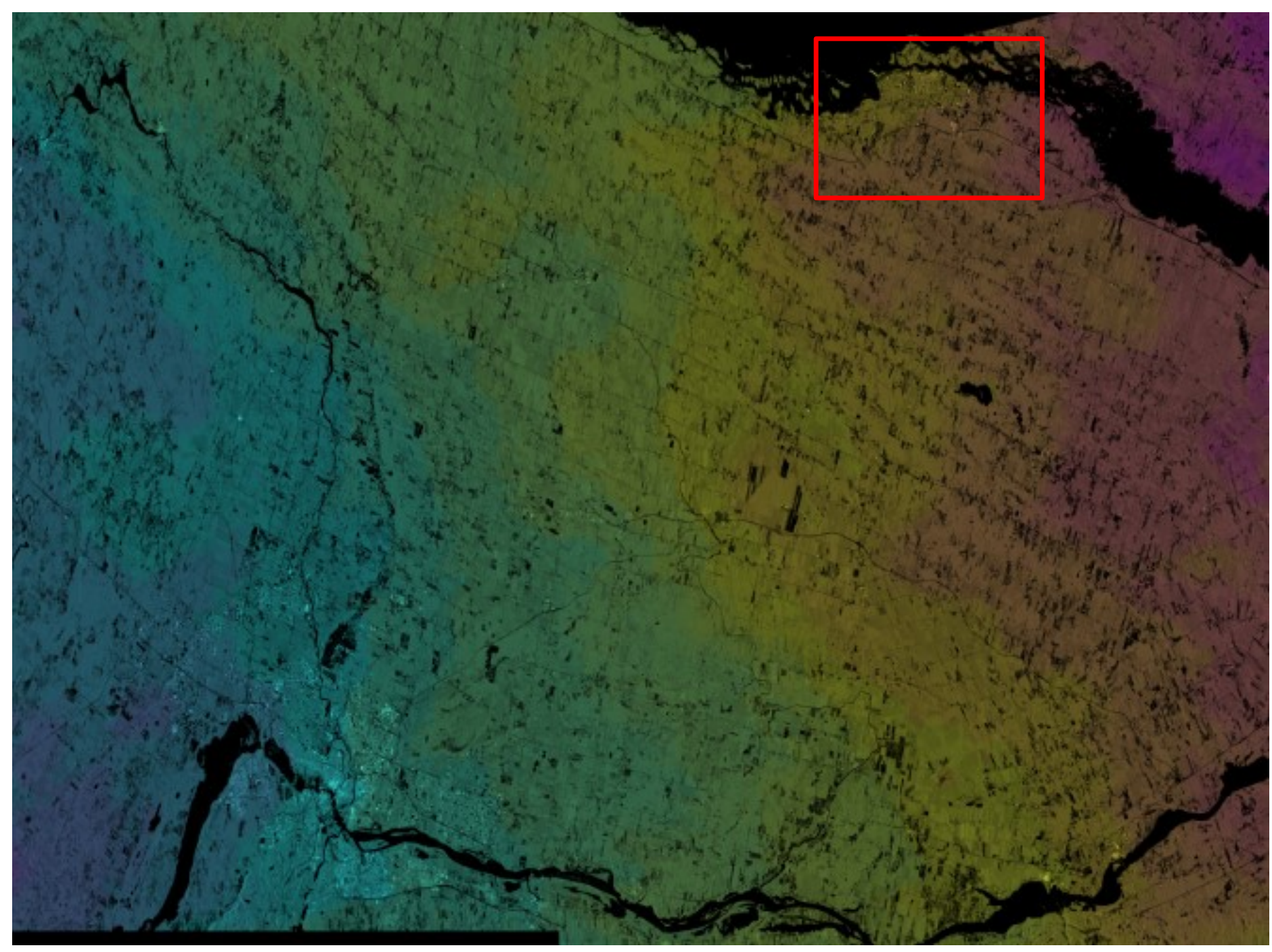

Figure 24: A filtered, unwrapped, differential interferogram (2015-10-06 to 2015-10-26) with a clear baseline related phase trend. Cornwall (red box) is clearly affected by the residual baseline trend, but this trend appears nearly constant within the red box. The interferogram is presented at $4 \pi$ per colour cycle. 
The suite of filtered, unwrapped, differential interferograms was examined and those with significant phase unwrapping errors or very low coherence were removed from further processing in the same way as in Section 5.1.1. The result was that 146 interferograms were selected for further analysis in iteration 2 (Figure 25). 


\section{Perpendicular baseline ( $\mathrm{m}$ ) vs. temporal baseline (days) for the Sentinel-1 dataset}

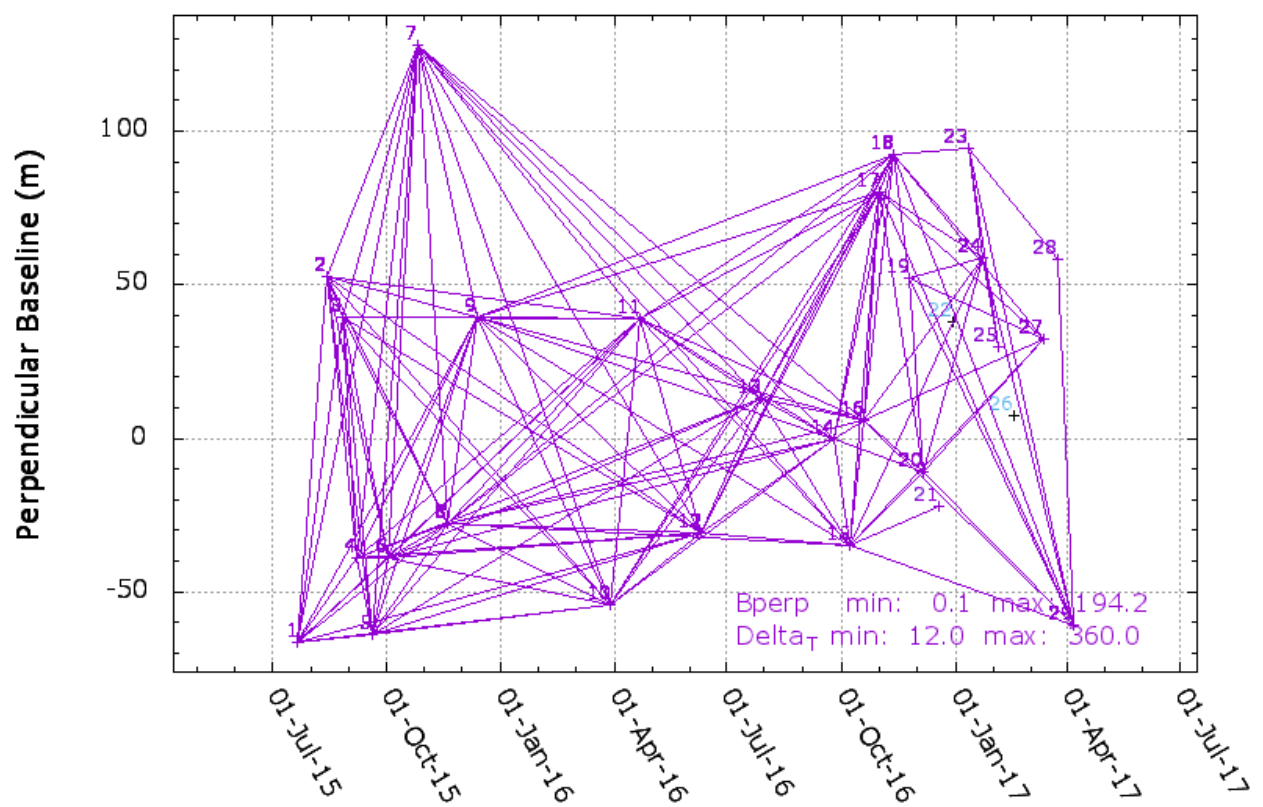

Figure 25: Perpendicular baseline $(\mathrm{m})$ vs. temporal baseline (days) for the interferograms from the Sentinel-1 suite selected for baseline refinement. 


\subsubsection{Iteration 2}

Iteration 2 consists of two stages. In stage 1, baseline refinement was performed on the interferogram suite established in iteration 1, and subsequently a new suite of baseline-refined, filtered, unwrapped, differential interferograms was then generated similarly to Section 5.1. Unlike for the RADARSAT-2 data (section 5.1.), the Sentinel-1 analysis was terminated prior to multi-baseline analysis. As discussed in section 7.2., a component of the interferometric phase difference not related to deformation prevents the application of the multi-baseline algorithm.

\section{Stage 1: Baseline refinement and interferogram generation}

The input to stage 1 is the suite of filtered, unwrapped, differential interferograms established in iteration 1. For each of these interferograms, GAMMA's baseline refinement procedure was applied; however, due to the exaggerated residual baseline phase trends no deformation mask was created. Following this, the differential interferogram suite was regenerated and filtering repeated. The filtering applied at this stage was softer than in iteration 1 , but still heavy enough to emphasize remaining baseline error. Phase unwrapping was performed with the same parameters as in iteration 1. In Figure 26 the same interferogram as in Figure 24, generated with refined baselines, is presented.

No further processing was performed on this interferogram suite. The magnitude of the unwrapped phase of these interferograms was significantly larger than could correspond to ground deformation; unwrapped phase values corresponding to several 
centimeters of deformation is observed even on interferograms with a small (e.g. 12 or 24 day) temporal baseline. This indicates that an IPD component, unrelated to ground deformation, was still prevalent after baseline refinement. In Section 6.2, several of the Sentinel-1 interferograms are presented (Figures 38 through 44) and subsequently discussed in Section 7.2. 

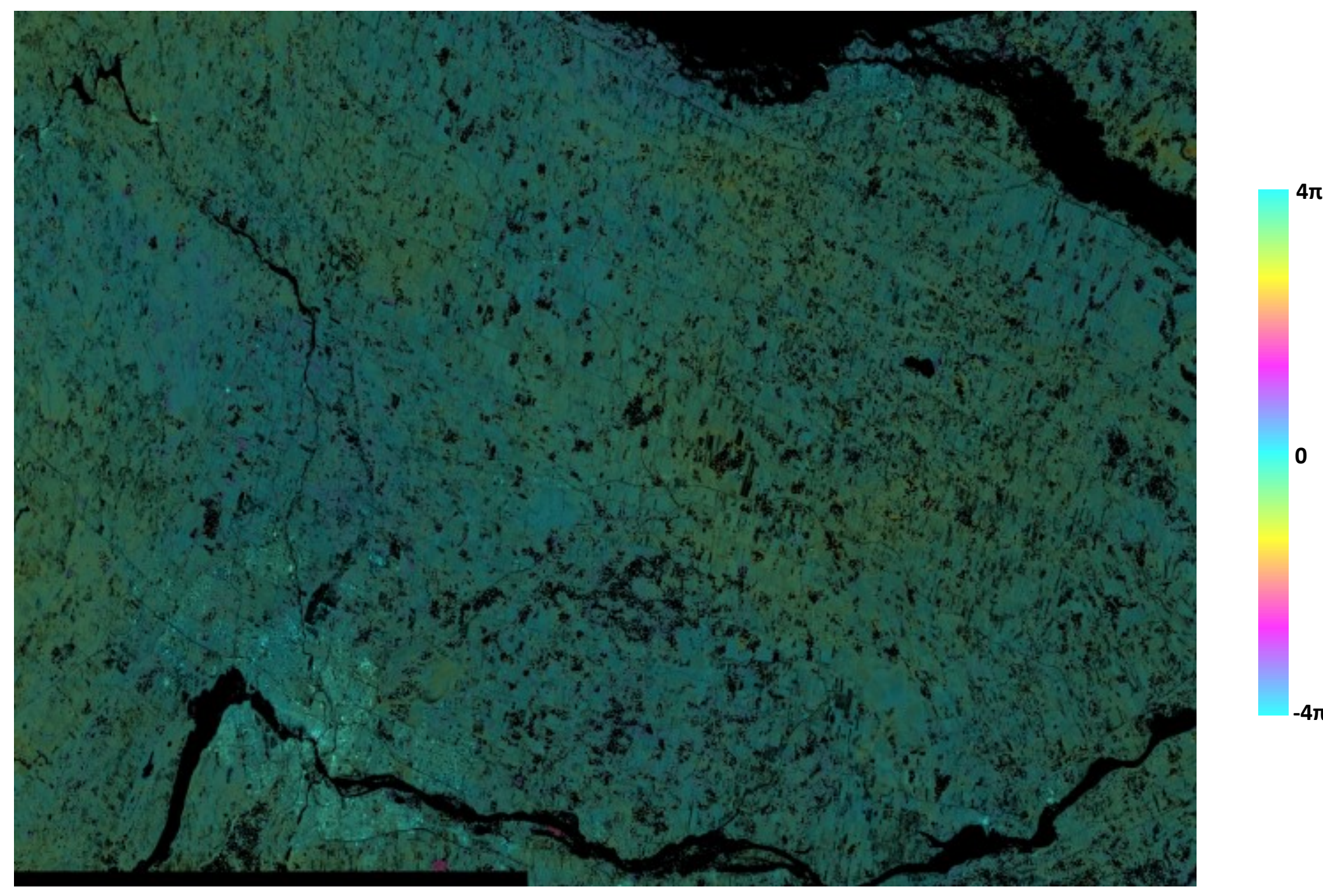

Figure 26: The interferogram from Figure 24 (2015-10-02 to 2015-10-26) regenerated with refined baselines. The prominent phase trend evident in Figure 24 has been entirely removed. The interferogram is presented at $4 \pi$ per colour cycle. 


\section{Chapter 6: Results}

In this chapter the results of the RADARSAT-2 and Sentinel-1 data processing are presented. For the RADARSAT-2 datasets sections of the cumulative deformation maps (i.e. the deformation from 2015-04-14 to 2016-09-23 for the SLA24 dataset, and from 2015-04-20 to 2016-09-05 for the SLA74 dataset) are shown in Figures 31 through 37. Due to the high resolution of the Spotlight mode acquisitions, cumulative deformation maps spanning the full study areas (Figure 11) cannot be displayed with reasonable resolution, and therefore subsets of this map (outlined in Figure 30) are shown instead.

\subsection{RADARSAT-2}

Prior to interpretation of the cumulative deformation maps, the error level (commonly referred to as the noise floor) must be determined. To do so, reference points that are assumed to be stable were examined. In Section 5.1.1., the use of the Cornwall Community Hospital as the stable phase unwrapping reference point was described, and it was noted that several other features (The R.H. Saunders Generating Station, Walmart Supercenter, The Home Depot, Canadian Tire, and Galaxy Cinemas) satisfy the same stability criteria. For these features, the InSAR derived time-series deformations should be equal to zero; the deviation from zero is the magnitude of the error at that specific point due to all contributions (see Section 7.1.). Table 6 shows the InSAR derived time-series deformations for each reference feature. 
Table 6: InSAR derived time-series deformations, UTM coordinates, and surficial soil of (assumed to be) stable reference points.

\begin{tabular}{|c|c|c|c|c|c|c|c|}
\hline \multirow{3}{*}{ 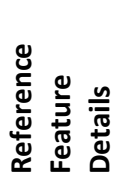 } & Feature & Hospital & $\begin{array}{l}\text { R.H. Saunders } \\
\text { Generating Station }\end{array}$ & Walmart & Home Depot & Canadian Tire & Galaxy Cinemas \\
\hline & \begin{tabular}{|l|} 
Location \\
(Easting/ Northing)
\end{tabular} & $522222 / 4986294$ & $516512 / 4983978$ & $520298 / 4985671$ & $519038 / 4987350$ & $521288 / 4986444$ & $523353 / 4985886$ \\
\hline & Surficial Soil & Diamicton & Diamicton & Diamicton & Diamicton & Sand & Diamicton \\
\hline \multirow{16}{*}{ 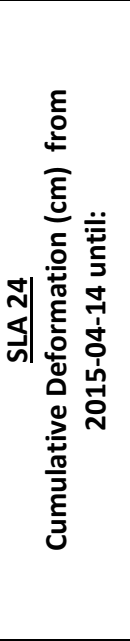 } & 2015-04-14 & 0.00 & 0.00 & 0.00 & 0.00 & 0.00 & 0.00 \\
\hline & 2015-05-08 & -0.06 & 1.21 & 0.10 & -0.61 & 1.10 & -0.41 \\
\hline & 2015-06-01 & -0.20 & -0.40 & -0.61 & -0.76 & 0.94 & -0.41 \\
\hline & 2015-06-25 & 0.24 & 2.31 & -0.96 & -0.66 & 0.98 & -0.68 \\
\hline & 2015-08-12 & 0.08 & 1.73 & 1.81 & -0.96 & -0.60 & -0.60 \\
\hline & 2015-09-05 & -0.06 & 2.54 & 2.50 & -0.17 & 0.72 & -0.55 \\
\hline & 2015-10-23 & -0.17 & 2.97 & 2.07 & -0.59 & 0.31 & -0.60 \\
\hline & 2015-11-16 & -0.64 & 2.99 & 2.51 & -0.63 & 1.00 & -0.40 \\
\hline & 2016-04-08 & -0.07 & 2.29 & 1.93 & -0.59 & 0.38 & -0.20 \\
\hline & 2016-05-02 & -0.08 & 2.61 & 1.91 & -0.92 & 0.37 & -0.08 \\
\hline & 2016-05-26 & 0.04 & 2.13 & 1.86 & -0.52 & 0.19 & -0.08 \\
\hline & 2016-06-19 & 0.00 & 2.78 & 2.16 & -0.86 & 0.30 & -0.16 \\
\hline & 2016-07-13 & 0.25 & 2.73 & 1.89 & -0.29 & 0.23 & -0.47 \\
\hline & 2016-08-06 & 0.03 & 3.28 & 1.61 & -1.12 & 0.07 & -0.61 \\
\hline & 2016-08-30 & 0.08 & 3.25 & 1.76 & -0.62 & 0.16 & -0.21 \\
\hline & 2016-09-23 & 0.01 & 3.35 & 1.80 & -0.81 & 0.68 & -0.04 \\
\hline \multirow{12}{*}{ 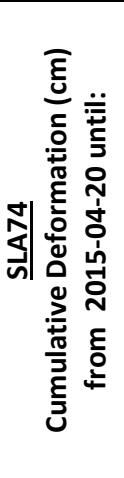 } & 2015-04-20 & 0.00 & 0.00 & 0.00 & 0.00 & 0.00 & 0.00 \\
\hline & 2015-06-07 & -0.01 & -2.07 & 0.04 & -0.29 & 0.77 & -1.10 \\
\hline & 2015-07-25 & 0.02 & -2.90 & -1.00 & -0.40 & 0.61 & -0.97 \\
\hline & 2015-08-18 & 0.00 & -2.09 & -1.07 & -0.51 & 0.78 & -0.99 \\
\hline & 2015-09-11 & 0.13 & -2.55 & -1.08 & -0.31 & 0.80 & -0.67 \\
\hline & 2016-03-21 & 0.08 & -1.12 & -1.69 & 0.12 & 0.88 & -1.02 \\
\hline & 2016-05-08 & 0.19 & -1.19 & -1.77 & -0.04 & 0.08 & -1.05 \\
\hline & 2016-06-01 & 0.21 & -0.89 & -1.83 & -0.14 & 0.80 & -1.23 \\
\hline & 2016-06-25 & 0.17 & -1.36 & -1.77 & -0.15 & 0.97 & -1.06 \\
\hline & \begin{tabular}{|l|}
$2016-07-19$ \\
\end{tabular} & 0.15 & -2.13 & -1.86 & 0.08 & 0.84 & -1.08 \\
\hline & \begin{tabular}{|l|}
$2016-08-12$ \\
\end{tabular} & 0.14 & -1.48 & -1.02 & 0.10 & 0.40 & -1.30 \\
\hline & 2016-09-05 & 0.96 & -2.55 & -1.50 & -0.69 & 1.51 & -1.54 \\
\hline
\end{tabular}


Figures 27 and 28, below, summarize the information presented in Table 6 . In Figure 27 the magnitude of the error levels of the reference points were averaged and plotted for each deformation map. These values are then the noise floors of their respective map: $\pm 1.0 \mathrm{~cm}$ and $\pm 1.5 \mathrm{~cm}$ for the SLA24 and SLA74 datasets, respectively. In Figures 31 through 37, the cyan colour is assigned to these ranges. Figure 29 shows histograms of pixel values for the cumulative deformation maps, with the noise floor range indicated. From these histograms it is clear that the majority of pixel values fall within the noise floor; this is discussed in Section 7.1. Similarly, the average error levels of the reference points through the time-series are presented in Figure 28, indicating which regions contribute to error the most. The spatial variation of error displayed in Figure 28 is discussed in Section 7.1. 


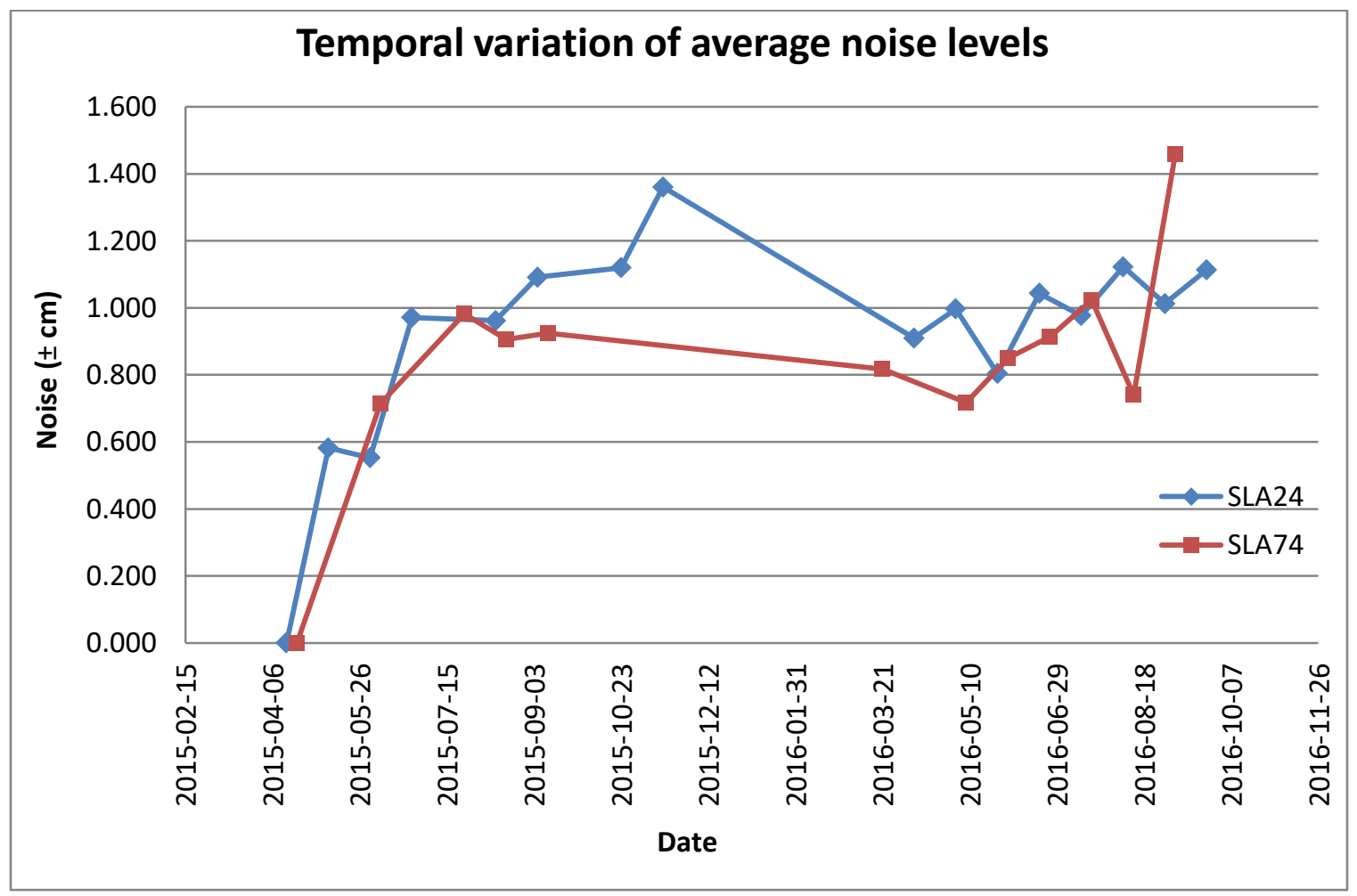

Figure 27: Average magnitude of the error levels of the reference points, for each deformation map. 


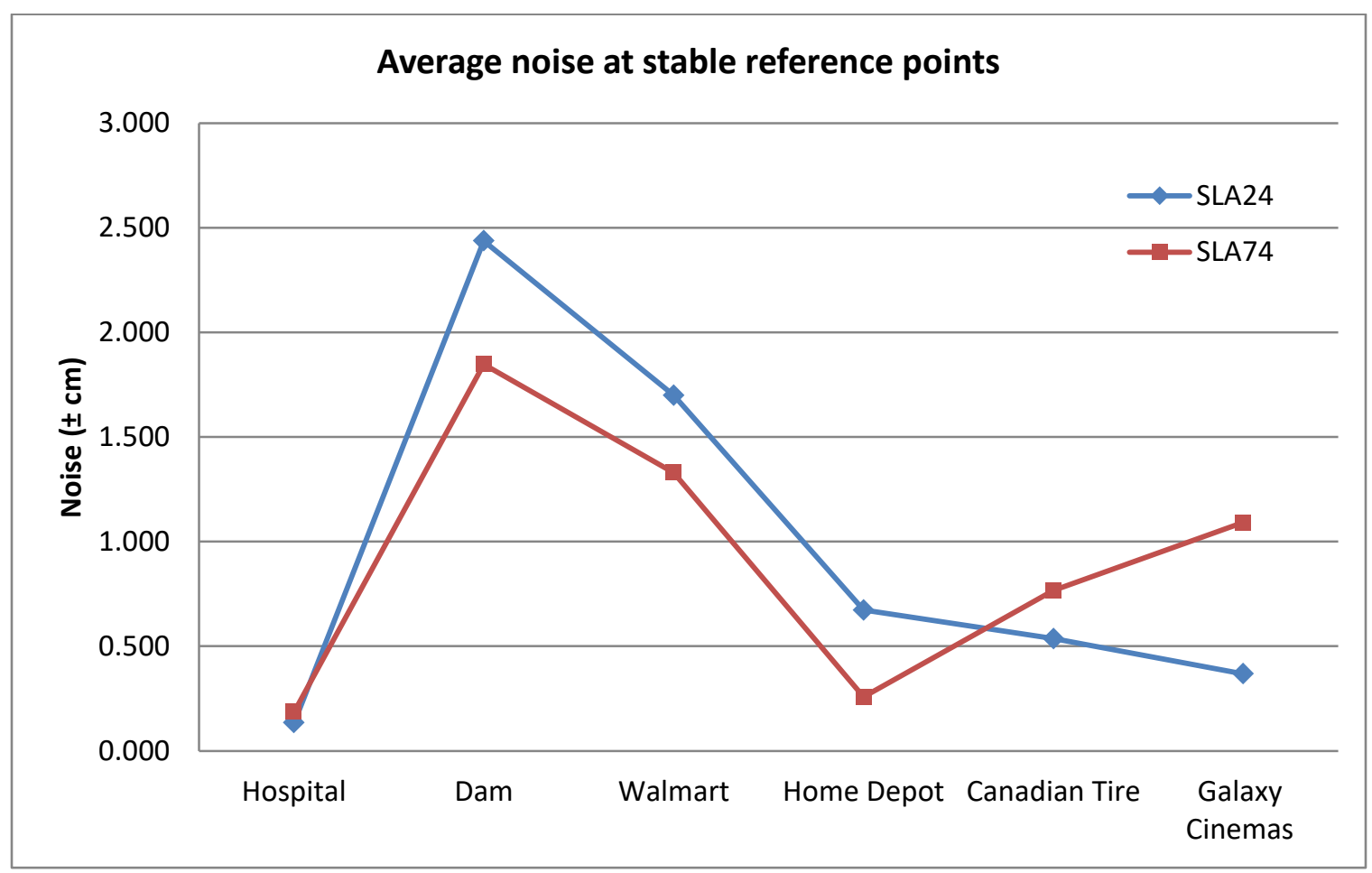

Figure 28: Average magnitude of the error level for each reference point throughout the time-series. 

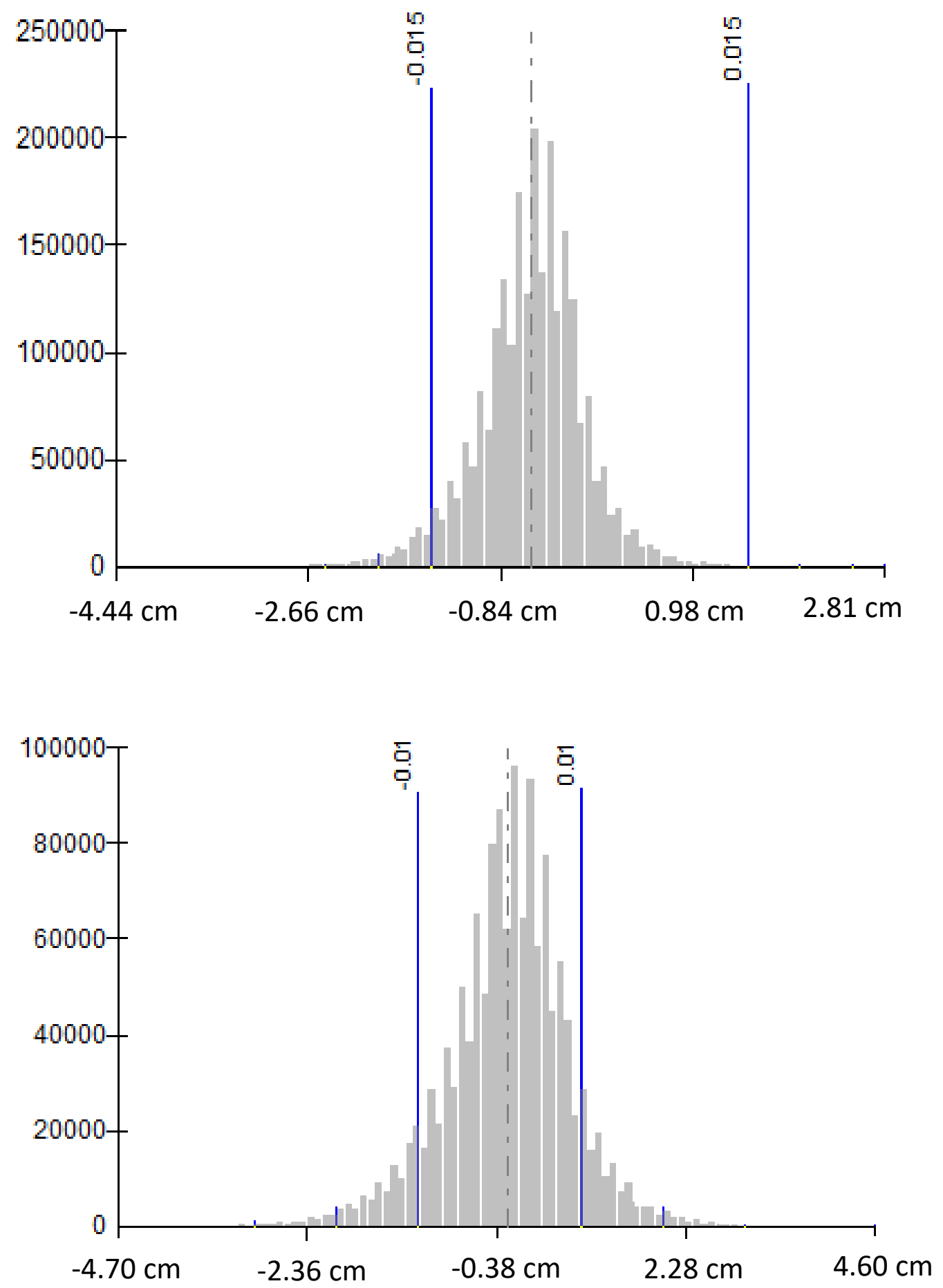

Figure 29: Histogram of pixel values, mean pixel value (dotted line), and noise floor levels for the (top) SLA74 and (bottom) SLA24 cumulative deformation maps. 


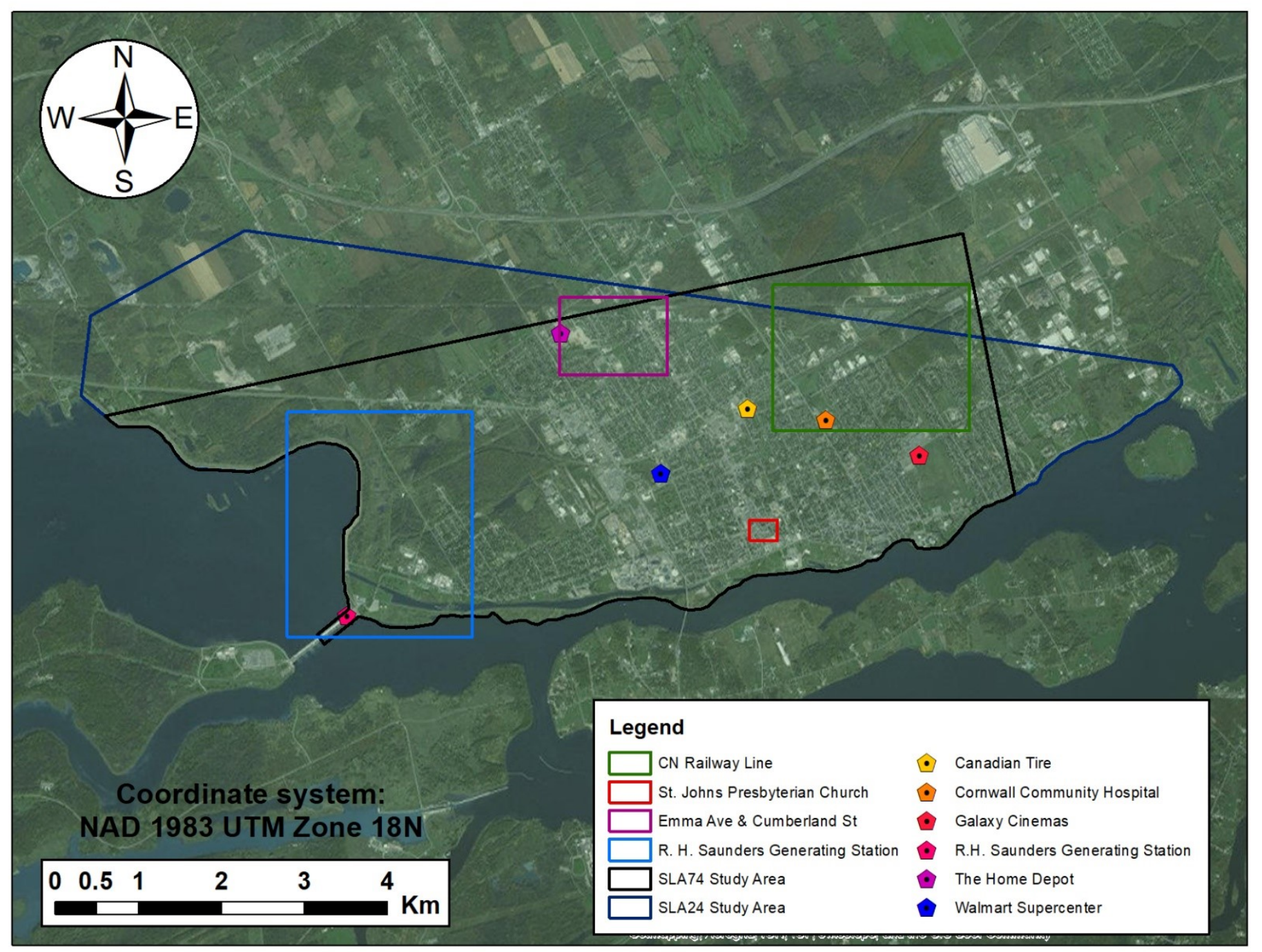

Figure 30: Map of Cornwall Ontario with the RADARSAT-2 study areas, reference point locations, and case study locations. 


\subsubsection{SLA74 Results}

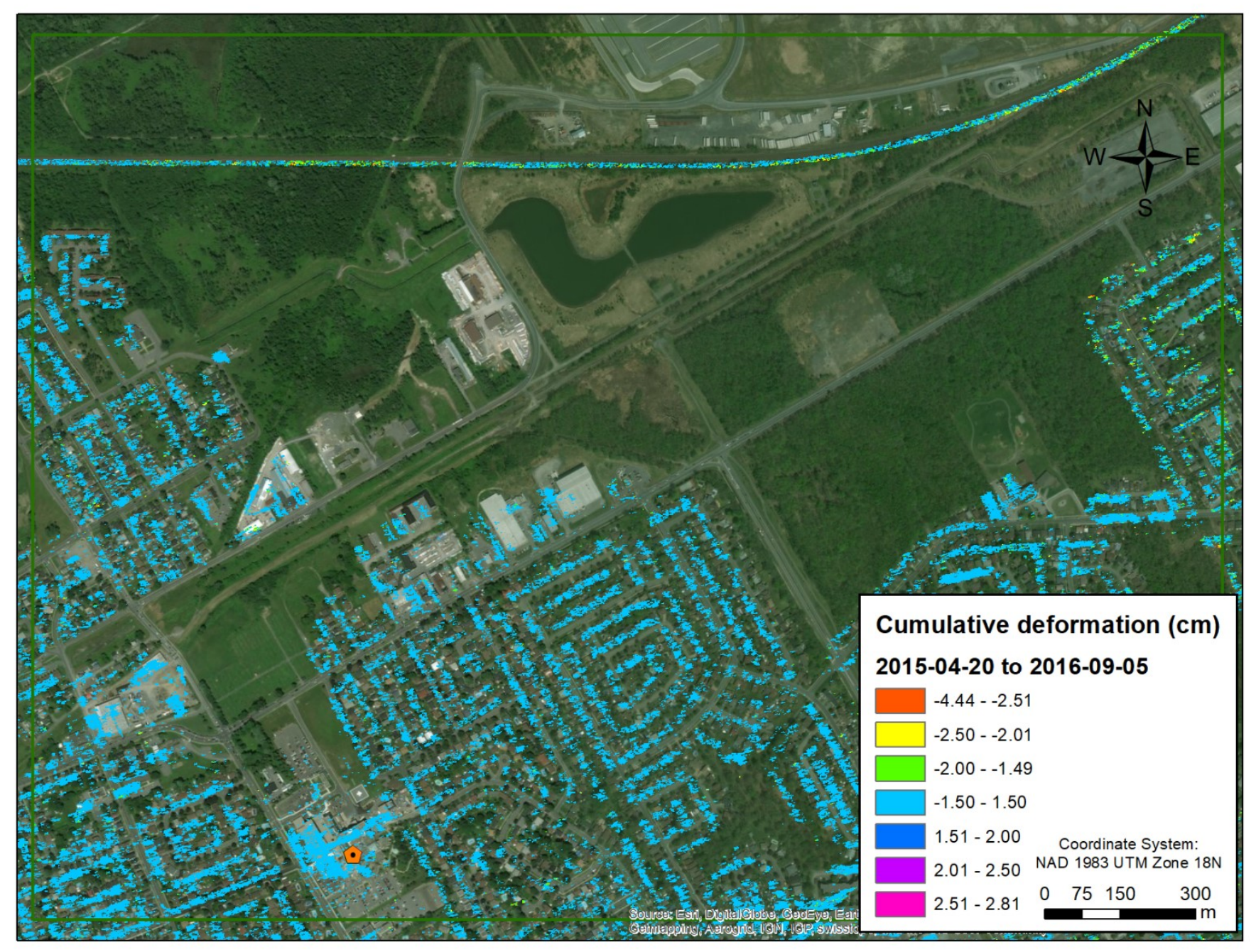

Figure 31: Deformation of the north eastern section of the CN Railway line derived from the SLA74 dataset. The location of the Cornwall Community Hospital is indicated by the orange pentagon. The extent of the green box is the same as in Figure 30 . 


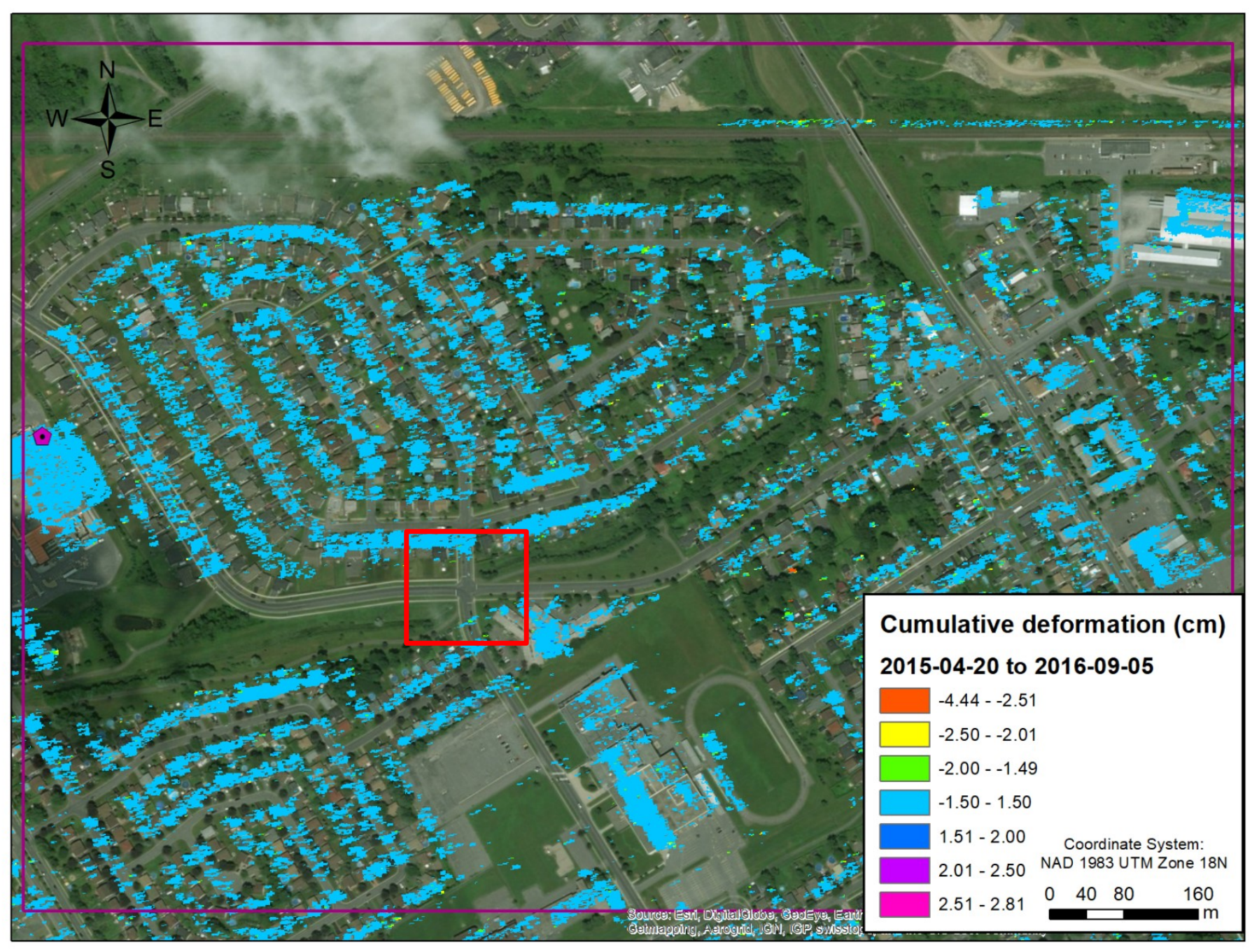

Figure 32: Deformation of the Emma and Cumberland intersection (red box) and surrounding area derived from the SLA74 dataset. The Home Depot used as a reference point is indicated by the pink pentagon. The extent of the pink box is the same as in Figure 30. 


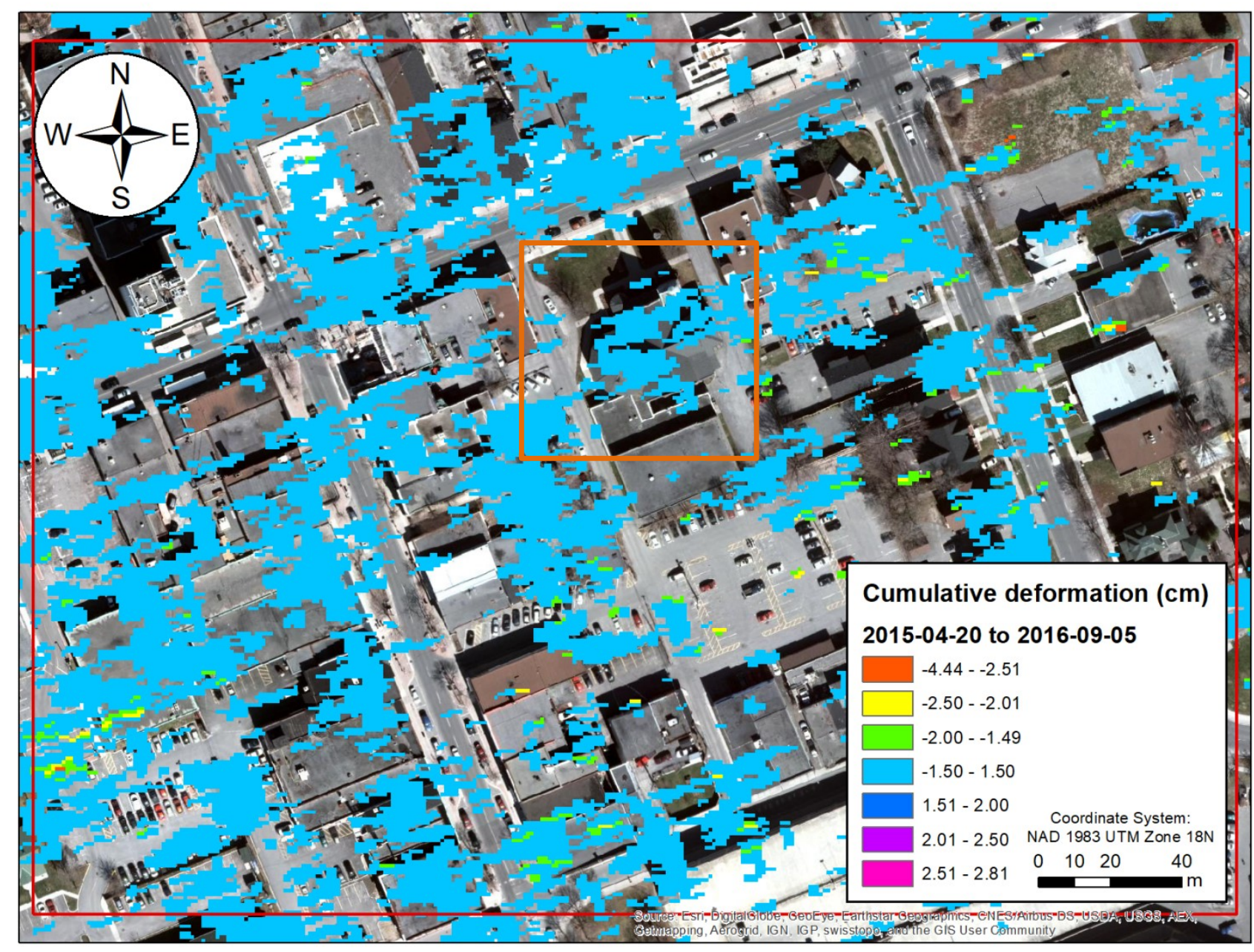

Figure 33: Deformation of the St. Johns Presbyterian Church (orange box) and surrounding area derived from the SLA74 dataset. The extent of the red box is the same as in Figure 30. 


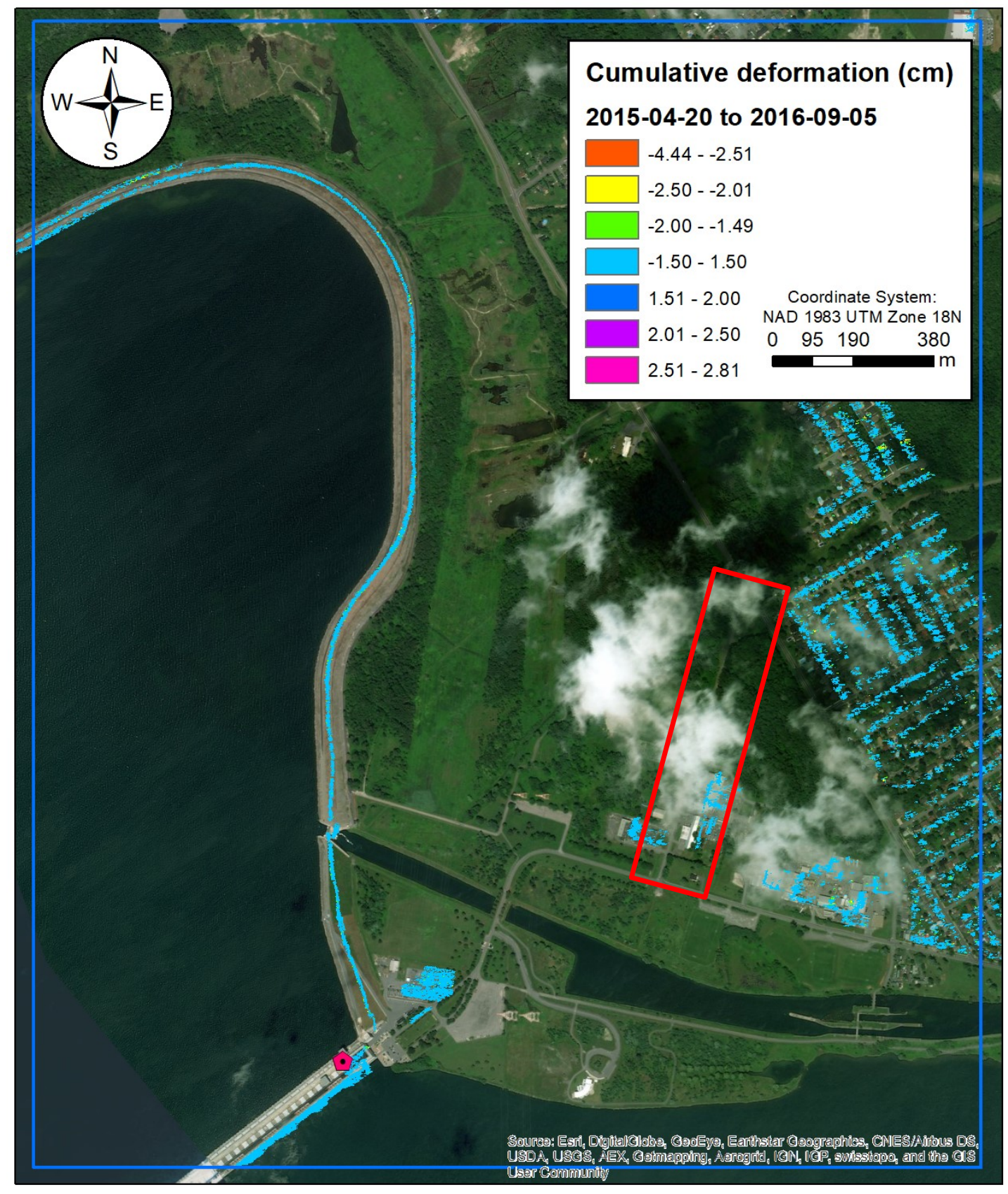

Figure 34: Deformation of the R.H. Saunders Generating Station's access road and surrounding area derived from the SLA74 dataset. The location of the R.H. Saunders Generation Station reference point is indication by the pentagon, and the red box surrounds Saunders Rd. The extent of the blue box is the same as in Figure 30. 


\subsubsection{SLA24 Results}

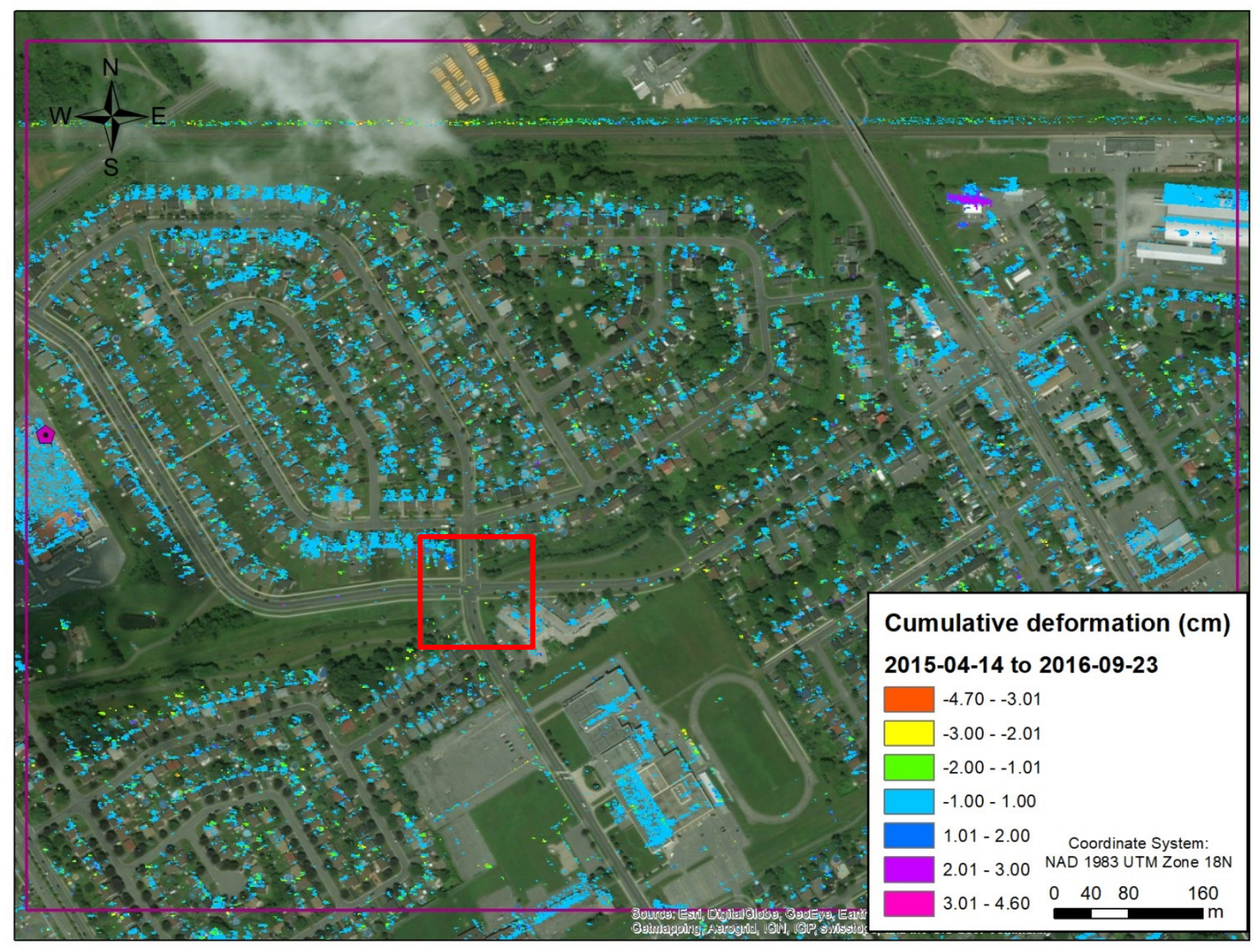

Figure 35: Deformation of the area surrounding the Emma and Cumberland intersection (red box) derived from the SLA24 dataset. The Home Depot used as a reference point is indicated by the pink pentagon. The extent of the pink box is the same as in Figure 30. 


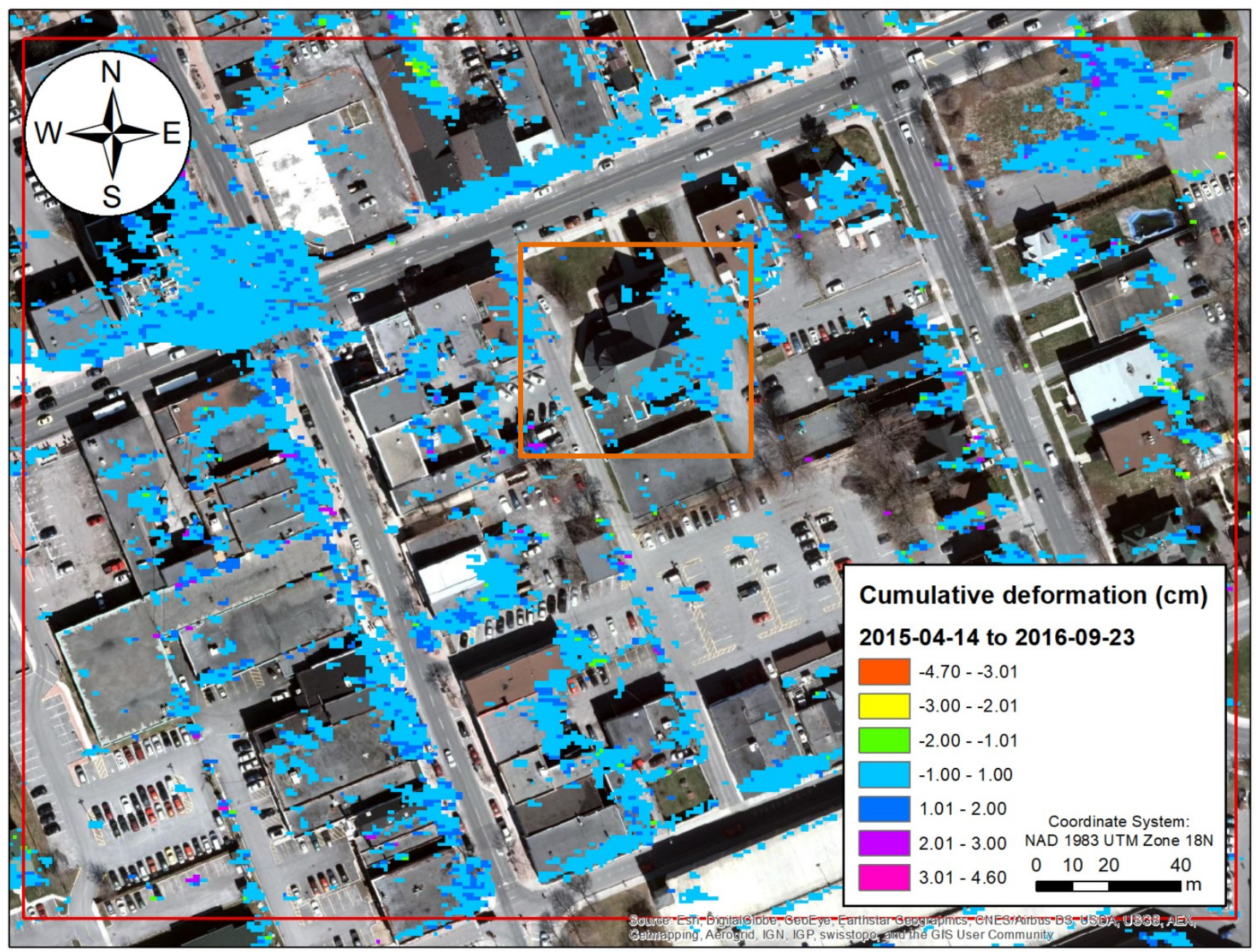

Figure 36: Deformation of the St. Johns Presbyterian Church (orange box) and surrounding area derived from the SLA24 dataset. The extent of the red box is the same as in Figure 30. 


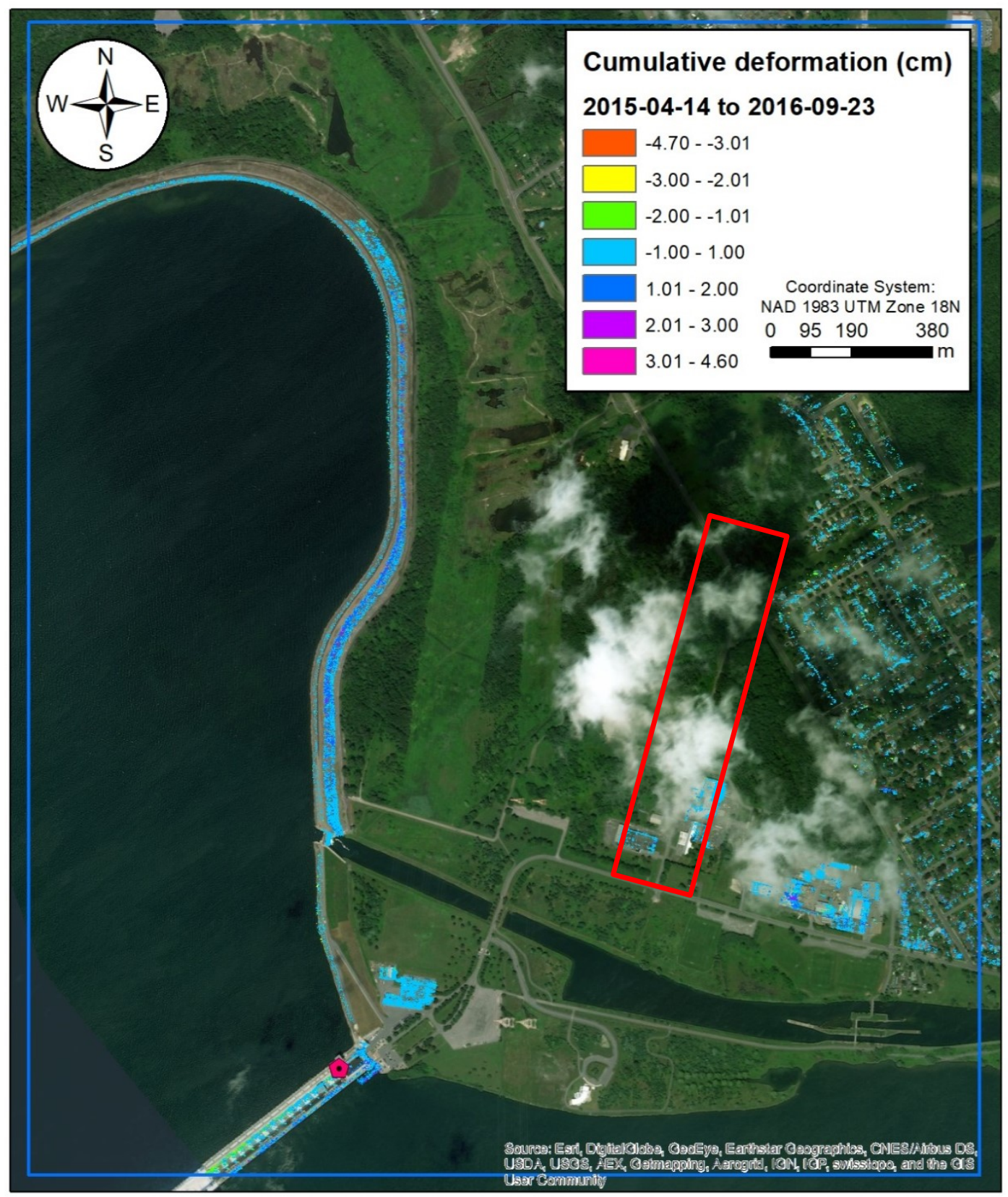

Figure 37: Deformation of the area surrounding the R.H. Saunders Generating Station's access road derived from the SLA24 dataset. The location of the R.H. Saunders Generation Station reference point is indication by the pentagon, and the red box surrounds Saunders Rd. The extent of the blue box is the same as in Figure $\mathbf{3 0 .}$ 


\subsection{Sentinel-1}

In this section five baseline refined, filtered, unwrapped, differential interferograms are presented. These interferograms were selected from the 146 created in iteration 2 (Section 5.2.2) to 1) demonstrate that an IPD component, not related to ground deformation, remains in the interferograms preventing the application of the multibaseline algorithm. 2) demonstrate that magnitude of the IPD is not strongly correlated to precipitation, 3) illustrate that the magnitude of the IPD is somewhat seasonally variable, 4) indicate which urban structures and linear infrastructure elements show potential for monitoring in terms of coherence levels and resolution, and 5) show variation in coherence with respect to time between acquisitions, seasons, and location within the study area. In Appendix C, five ancillary interferograms are presented as additional examples to those shown below. 


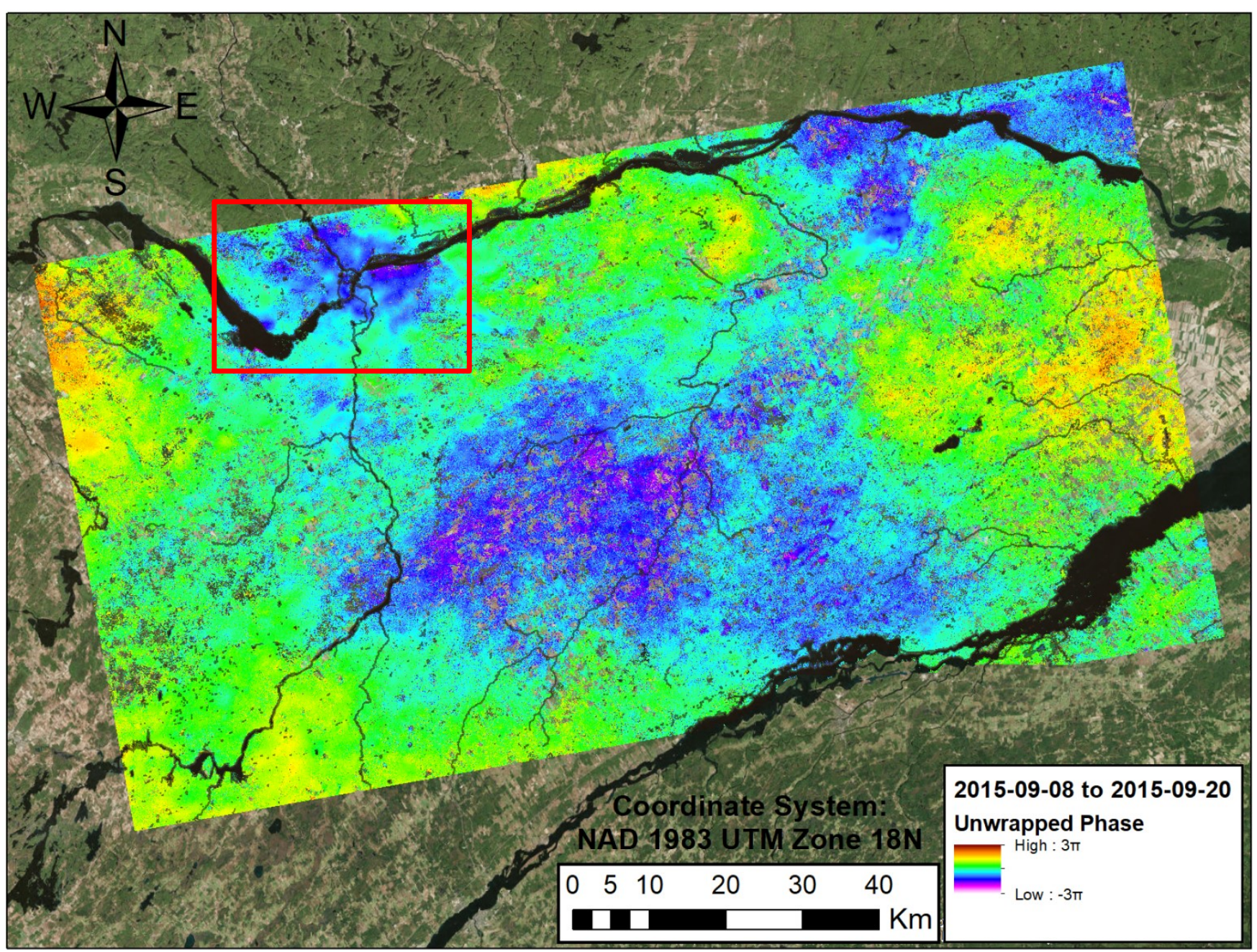

Figure 38: Unwrapped, differential interferogram created from Sentinel-1 summer acquisitions (2015-09-08 and 2015-09-20) with a 12 day temporal baseline. The red box indicates the approximate area in which precipitation was occurring on 2015-09-08 at the time of acquisition. 

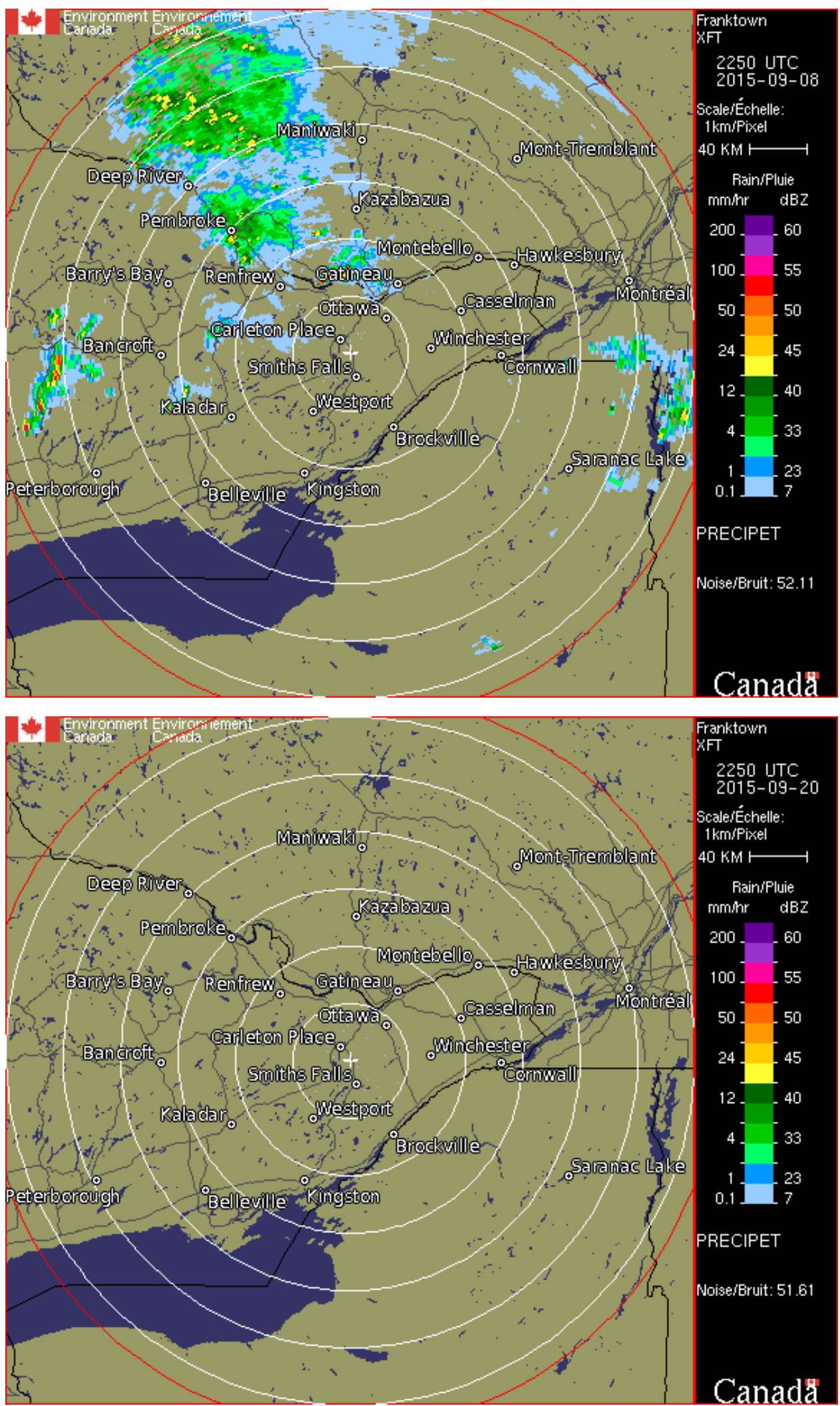

Figure 39: Weather radar images showing precipitation in the study area at 22:50 UTC on (top) 201509-08 and (bottom) 2015-09-20. 


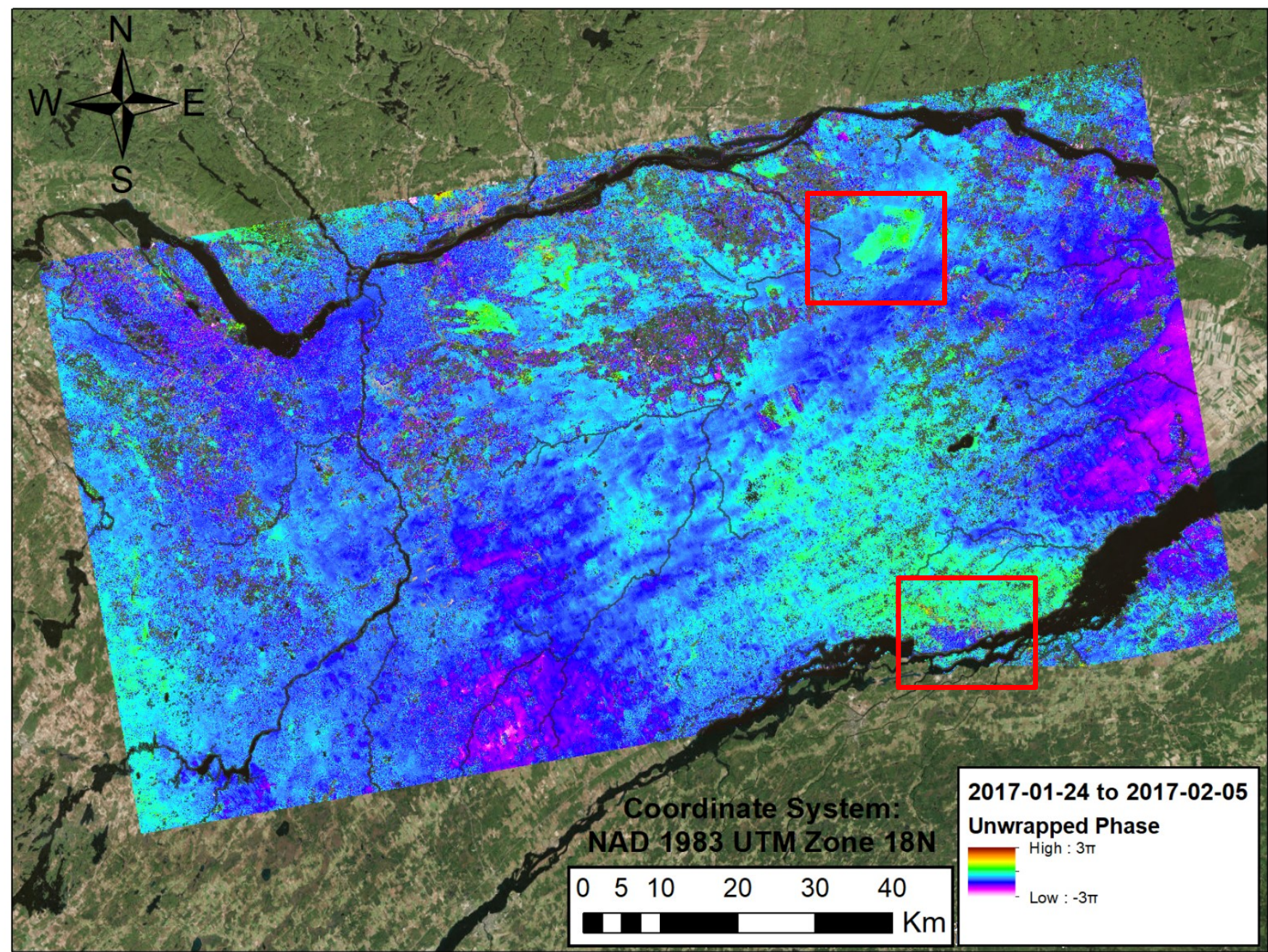

Figure 40: Unwrapped, differential interferogram created from Sentinel-1 winter acquisitions (2017-01-24 and 2017-02-05) with a 12 day temporal baseline. The areas bounded by the red boxes are presented in Figure 41 below. 

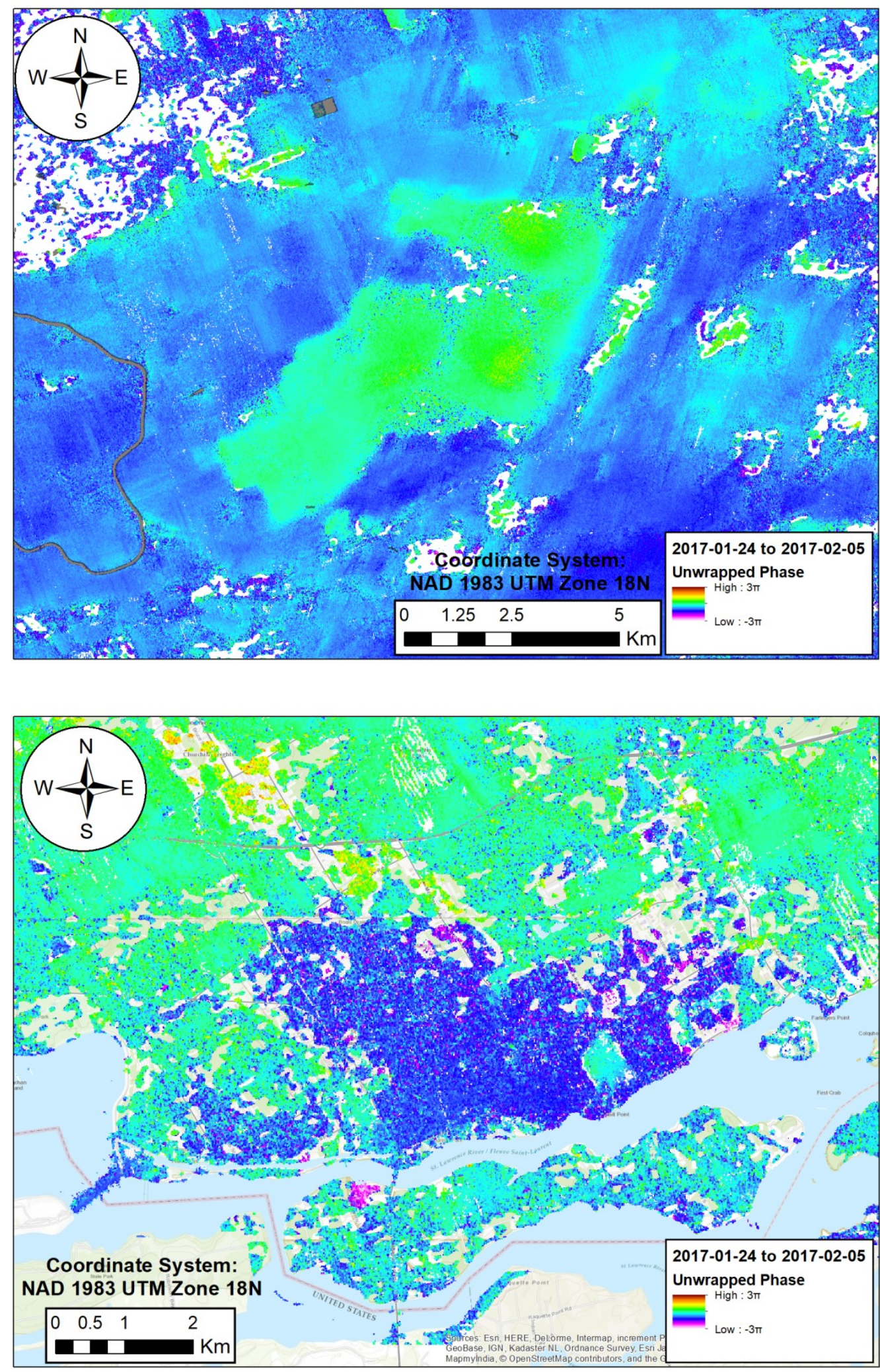

Figure 41: Sections of the unwrapped, differential interferogram in Figure $\mathbf{4 0}$ derived from the Sentinel-1 dataset; (top) Alfred's bog, and (bottom) the city of Cornwall. 


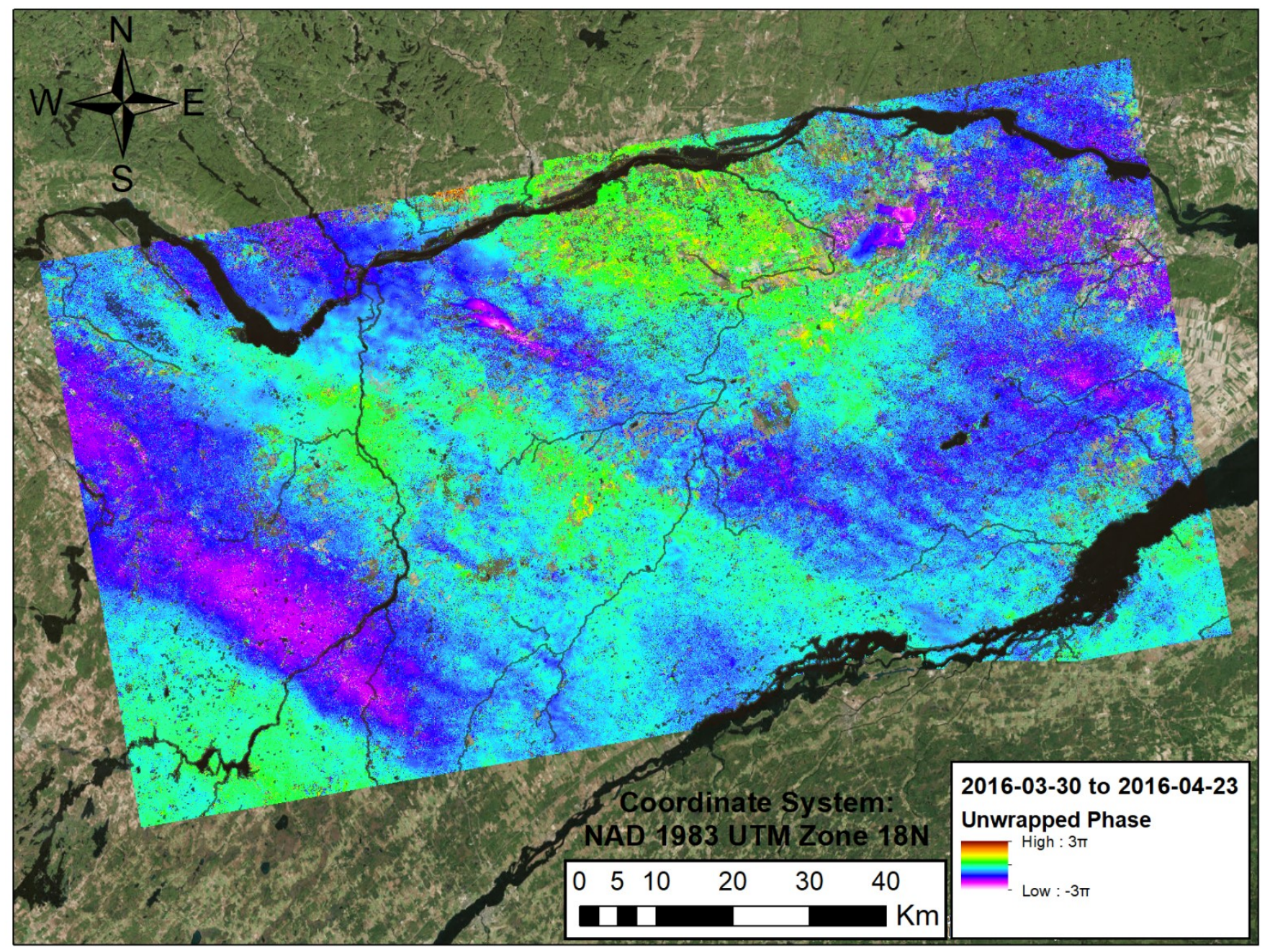

Figure 42: Unwrapped, differential interferogram (2016-03-30 and 2016-04-23) created from Sentinel-1 spring acquisitions with a 24 day temporal baseline. 


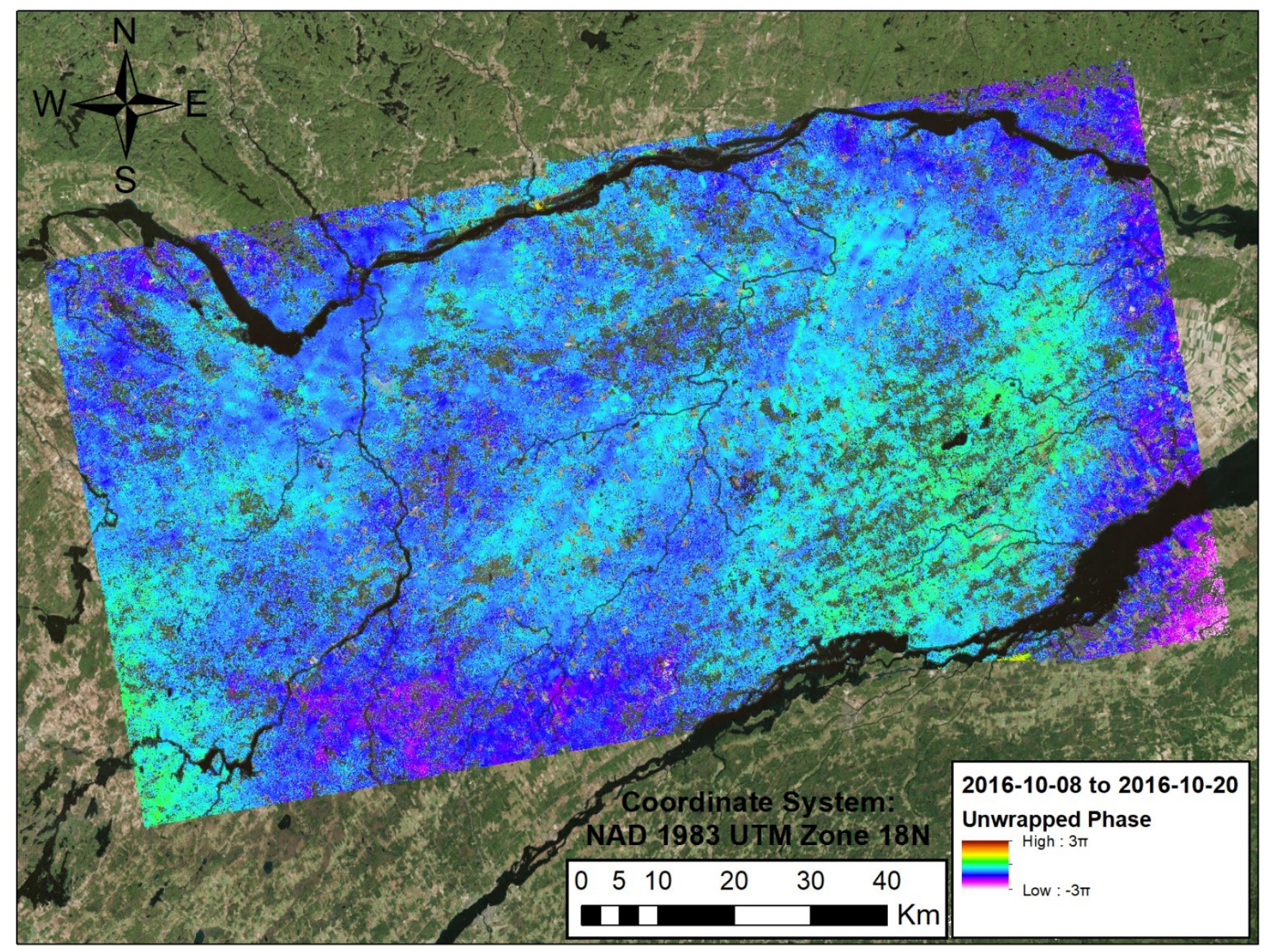

Figure 43: Unwrapped, differential interferogram created from Sentinel-1 fall acquisitions (2016-10-08 and 2016-10-20) with a 12 day temporal baseline. 


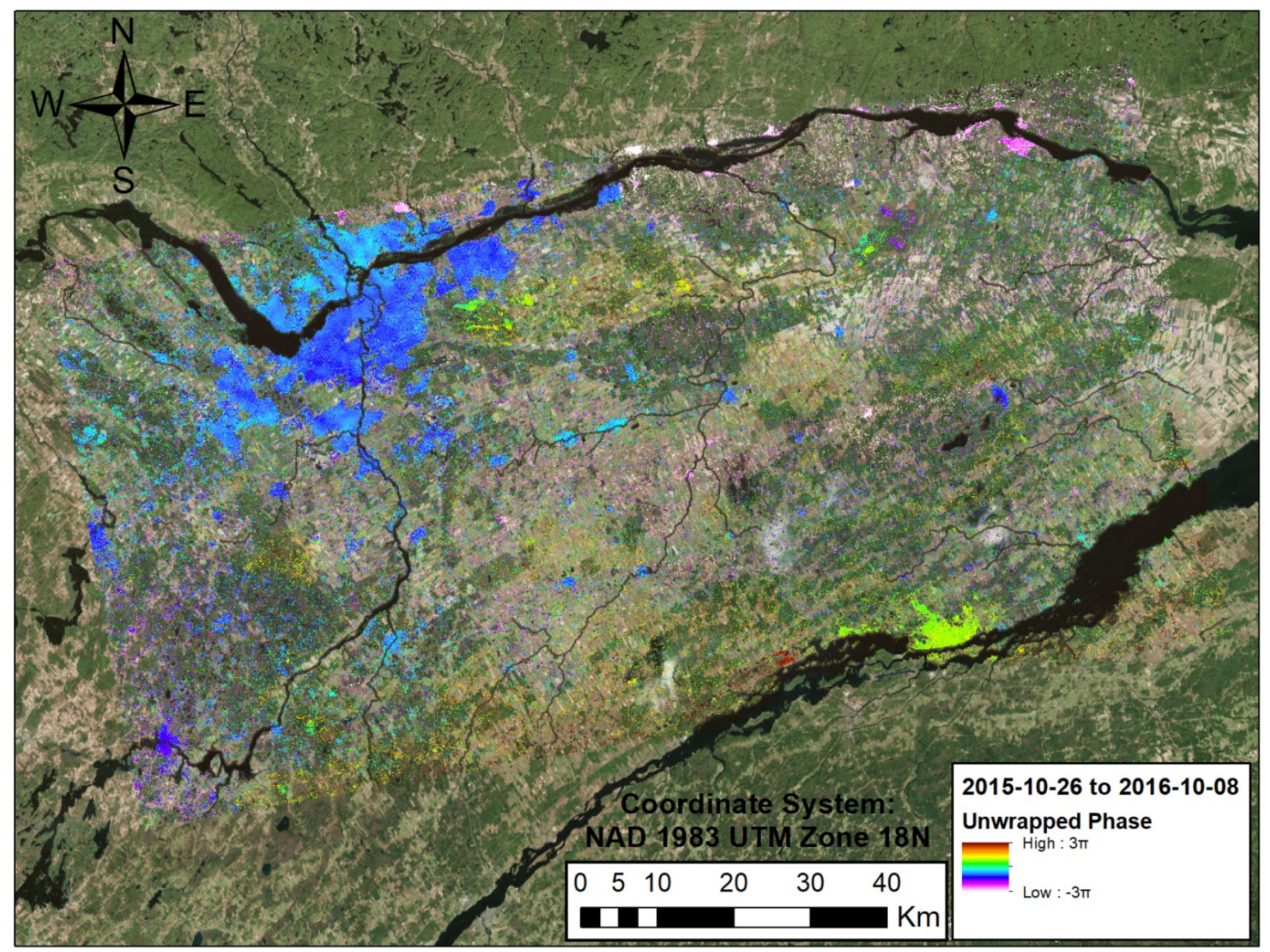

Figure 44: Unwrapped, differential interferogram created from Sentinel-1 fall acquisitions (2015-10-26 and 2016-10-08) with a 348 day temporal baseline. 


\section{Chapter 7: Discussion}

This section holds a discussion of the results of the processing of the RADARSAT-2 and Sentinel-1 datasets. From Section 6.1 it is clear that for the RADARSAT-2 datasets, the noise floor - estimated to be $\pm 1.0 \mathrm{~cm}$ and $\pm 1.5 \mathrm{~cm}$ for the SLA24 and SLA74 datasets, respectively - is significantly greater than not only the expected deformation rate in Cornwall, but also the noise floors determined by other studies (e.g. $0.2 \mathrm{~cm}$ for Henschel et al. 2015). The discussion in Section 7.1 is centered primarily on describing the differences between the results obtained with different datasets, presenting challenges in interpreting InSAR derived deformation maps, and secondarily on identifying factors which contribute to the substantial noise floor. Both discussion points are supported by an examination of the RADARSAT-2 derived deformation maps of the case studies described in Section 4.2.1. In Section 7.2., the Sentinel-1 interferograms are discussed with a major focus on attempting to constrain the unidentified interferometric phase component. Additionally, the potential for infrastructure monitoring with this dataset is discussed in terms of image resolution and coherence.

\subsection{RADARSAT-2 Discussion}

In Section 4.2.1., several case studies were introduced. A two-track portion of the $\mathrm{CN}$ railway line, the St. Johns Presbyterian church, two sections of city streets, and the R.H. Saunders Generating Station's access road were selected for monitoring. InSAR derived deformation maps of these case studies were presented in Section 6.1. Below, the deformation maps of these case studies are described. 


\subsubsection{CN Railway Line}

Figures 31, 32, and 35, show the InSAR derived deformation of sections of the CN railway line. For both datasets, the railway line is a continuously coherent feature spanning the entire study area. As indicated by the coherence mask in Figure 20, no section of the railway displays low or transient coherence levels in the interferogram suite. The railway line consists of two steel tracks built on a relatively shallow embankment (compared to the embankment of the R.H. Generating Station's access road, for example) constructed of primarily of gravel. The railway line is somewhat removed from the city center such that no portion of the track is obscured by radar shadow. Furthermore, the vegetation in the immediate vicinity of the railway line is maintained via a vegetation management program such that shrubs and trees do not grow on or immediately adjacent to the track. As such, the railway line is a linear feature constructed from material that results in high interferometric coherence, and is free of radar shadow or vegetation induced coherence loss.

The majority of pixels representing the railway line show deformations within the noise floor. Pixels showing deformation outside of the noise floor tend to be arranged either in small clusters (as is most clearly seen in Figure 31) that show very rapid deformation with respect to the rest of the railway, or as individual pixels showing rapid deformation surrounded by pixels contained within the noise floor. Neither of these situations corresponds to a plausible physical scenario. A railway line experiencing subsidence or creep would be expected to have deformation that smoothly varies in magnitude along the track. The cluster of green-red pixels in Figure 31 spans the full 
width of the track, and indicates subsidence of about $2-4 \mathrm{~cm}$. However, this cluster is immediately adjacent on either side to pixels within the noise floor. For a railway line constructed from hot rolled steel, this magnitude of deformation occurring over a span of a few meters is unlikely. Moreover, any real deformation would be expected to be present in both datasets, which comparing Figures 32 and 35 demonstrates is not the case. With this in mind, the InSAR derived deformation of the railway line is attributed to residual phase unwrapping errors along the railway line.

The phase unwrapping begins at the phase reference point, in this case the Cornwall Community Hospital which is located somewhat centrally within the city, and progresses away from that point. In order to unwrap the railway line, the phase unwrapping algorithm has to cross the relatively vegetated area that is littered with industrial equipment, located between the main city center and the railway line. The quality of phase unwrapping of the railway line was particularly sensitive to the phase unwrapping mask used. In order to minimize phase unwrapping errors, careful attention was paid when making the phase unwrapping mask to completely remove all pixels in between the main city center and the railway line.

The Spotlight mode data used in this project is of very high spatial resolution. Because of this high resolution, the interferometric phase difference arising from small, transient features within a particular ground resolution cell proportionally affect the phase of the corresponding SAR pixel more so than in coarser resolution datasets. In an urban setting many small features that provide strong radar returns and maintain 
interferometric coherence (e.g. street signs) are present. Such features, however, often deform rapidly, without any geotechnical cause. The assumption of slow phase variation between adjacent or nearby pixels made by most phase unwrapping algorithms is therefore not maintained; in an urban setting the interferometric phase difference of neighbouring pixels can vary significantly, and deformation information contained within these pixels are often not geotechnically relevant.

\subsubsection{St. Johns Presbyterian Church}

Figures 33 and 36 show the SLA74 and SLA24 InSAR derived deformation of the St. John's Presbyterian Church. In both of these deformation maps, clusters of pixels corresponding to a portion of the church are present, indicating that the church (as with most buildings) maintains suitably high coherence for deformation monitoring to be performed; the building is not hidden in radar shadow or heavily obscured by foliage and, as demonstrated in Figure 45, strongly backscatters the radar signal making it an intense (radar bright) feature in MLI images from both datasets. Furthermore, the church is the smallest feature of the case studies, being only approximately $25 \mathrm{~m} \times 40 \mathrm{~m}$ in dimension, but is clearly well within the resolution capabilities of the Spotlight mode data. The nominal spatial resolution of approximately $3 \mathrm{~m}$ allows for deformation of this small feature to be measured by clusters of independent pixels, and as such could be used to monitor buildings for relatively complex deformations such as differential settlement. In contrast to the railway line, no obvious phase unwrapping errors are present in either dataset. 
From Figures 33 and 36 three observations regarding the interpretation of InSAR derived deformation maps are made. The first observation is that in both figures the pixels representing deformation of the church are somewhat displaced from the church itself. In Figure 36 the pixels representing the southeastern aspect of the roof are shifted by a small amount (less than $10 \mathrm{~m}$ ), approximately northeast of their true position as indicated in the areal image. In Figure 33 this shift is more pronounced, with the pixels representing the northwestern aspect of the roof church displaced (by about $35 \mathrm{~m}$ ) to the southwest. In Figure 33, the pixels directly overlying the church actually represent a building located northeast of the church. These pixels have been similarly displaced, and coincidentally happen to fall on top of the church. The difference in position between pixels representing deformation and the true position of the church is not due to inaccurate geocoding or georeferencing; rather, it is the result of the SAR imaging geometry. Buildings are topographic features with vertical slopes, and therefore are strongly affected by layover (Section 2.2.).

Layover causes the pixels representing topographic features to be shifted towards the sensor. The SLA24 dataset was acquired with a right-looking, descending geometry and so the layover is in the approximately northeastern direction. Likewise, the SLA74 dataset was acquired with a right-looking, ascending geometry and so layover is in the approximately southwestern direction. The magnitude of the shift is inversely proportional to the incidence angle, and proportional to the height of the topographic feature. Layover is prevalent in every deformation map presented in Section 6.1. When interpreting these maps, care must be taken to account for the layover. Figure 46, 
below, shows an additional example where the layover is more clearly identified, due to the shape of the building, than in the St. Johns Presbyterian church example. Note that in this figure the colours do not correspond to deformation, and instead simply differentiate the SLA24 and SLA74 datasets. Layover shifts the data towards the respective sensor. Since the incidence angle of the SLA74 dataset is significantly smaller than that of SLA24, the resultant layover is much more significant.

The second observation made from Figures 33 and 36 is that the SLA74 dataset contains significantly more pixels than the SLA24 dataset (i.e. the SLA74 response is more intense). The third observation is that in neither dataset is the full church structure being monitored. For the SLA74 dataset the northwest portion of the roof is bright with radar returns whereas the southeastern portion is empty, and conversely for the SLA24 dataset. Both of these observations are again tied to the radar imaging geometry, in particular the pass type (ascending or descending), and the incidence angle.

Since the SLA74 dataset was acquired on an ascending pass and is right-looking (such that the signal is transmitted from west to east), topographic features with an eastern aspect tend to be hidden in radar shadow (and conversely for SLA24). Moreover, since the SLA74 incidence angle is smaller less of the topographic features are hidden in radar shadow, and likewise, the proportion of the transmitted signal that is backscattered by buildings is greater. The SLA74 MLI image in Figure 45 is significantly brighter than the SLA24 MLI image for this reason. Care must then be taken when 
comparing deformation maps from different passes since for features with extreme topography such as buildings, different sides of the feature will be monitored. The observations presented here suggest that the combination of ascending and descending data to retrieve 3D deformation for buildings is not possible. Unless two datasets with small incidence angles are used, the radar response will be from different sections of the building. However, if small incidence angles are used, then layover will become significant and displace the radar responses from the different datasets in opposite directions. 

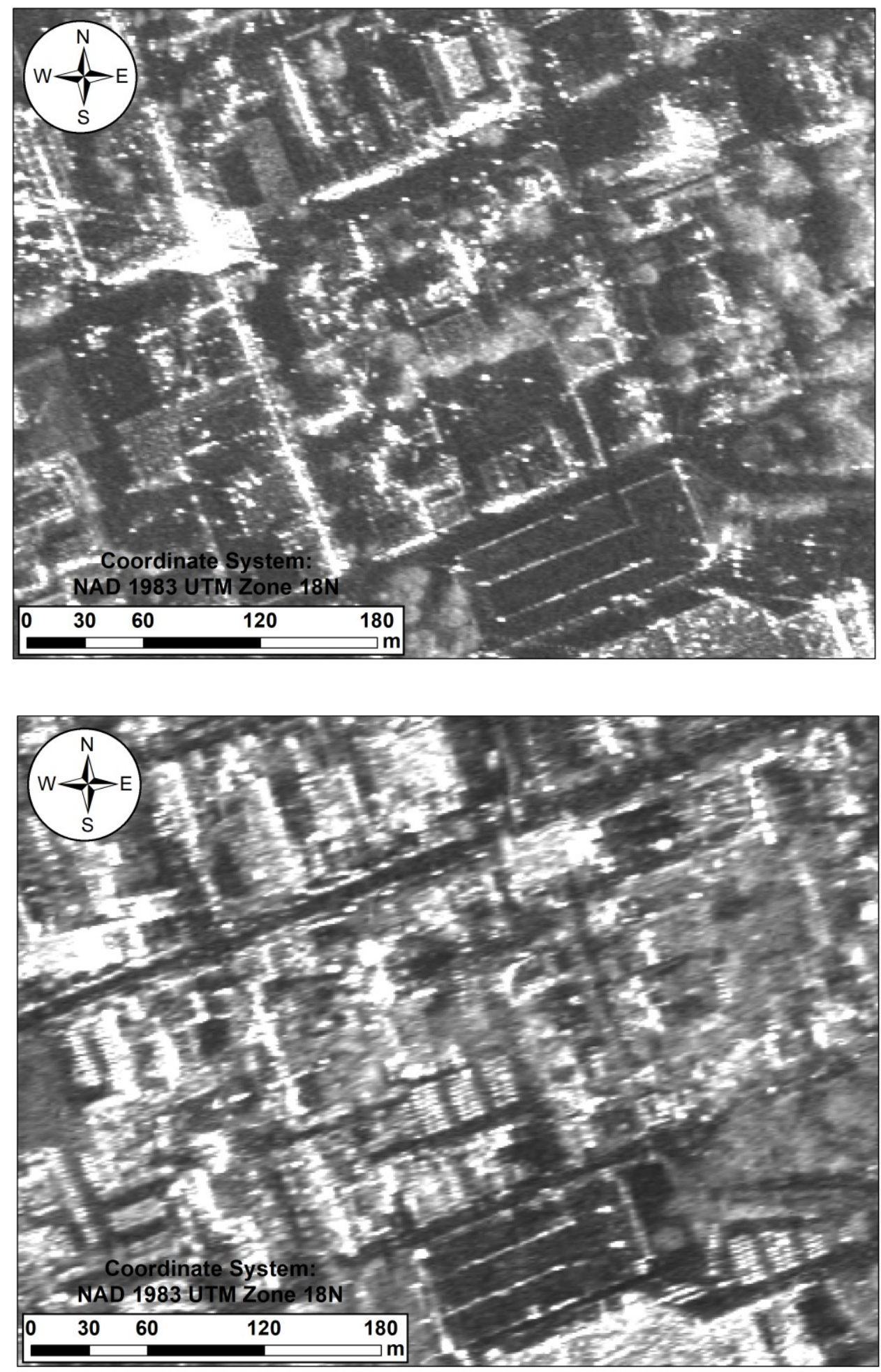

Figure 45: MLI images of the St. Johns Presbyterian Church from (top) the SLA24 dataset and (bottom) the SLA74 dataset. 


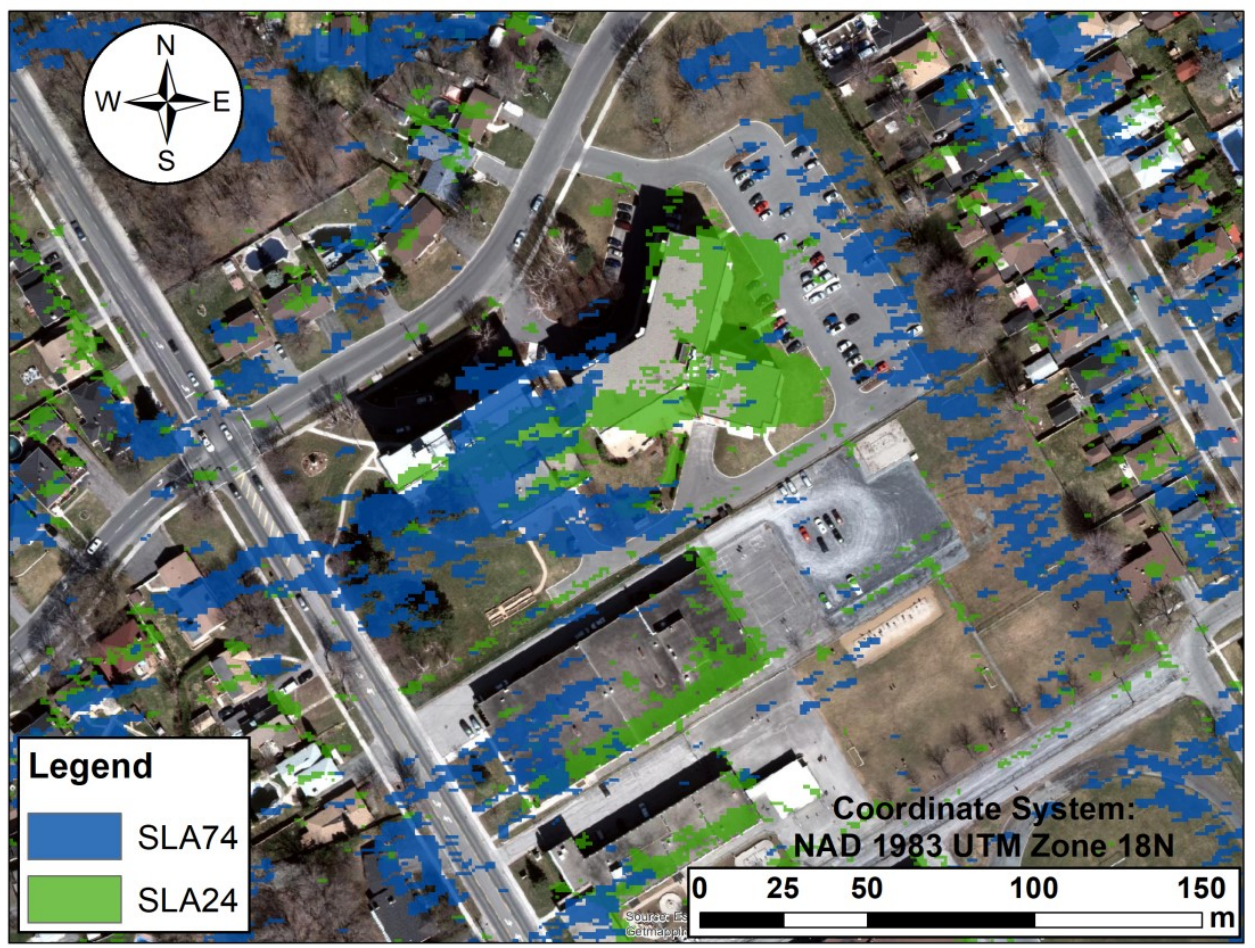

Figure 46: Layover in the (green) SLA24 and (blue) SLA74 datasets. 


\subsubsection{Intersection of Emma Ave and Cumberland St, and Saunders Rd}

Figures 32, 34, 35, and 37 show the InSAR derived deformation within the vicinity of the intersection of Emma Ave and Cumberland St, and Saunders Rd, for both datasets. In each of these figures it is clear that the road surface did not maintain suitable coherence for direct deformation monitoring to be performed. This same effect was observed in Figures 31, 33, and 36. The MLI images of Figure 45 show that road surfaces also have very low intensity. RADARSAT-2 operates in C-band, i.e. it transmits a signal with a wavelength of $5.6 \mathrm{~cm}$. Relative to this wavelength asphalt road surfaces are smooth, and therefore the backscattered proportion of the transmitted signal is small, whereas the reflected proportion is large. Because of the predominance of reflection rather than backscattering, C-band radar cannot be used to directly monitor road surfaces. Instead, deformation or degradation of asphalt road surfaces must be determined indirectly by looking at coherent features in the vicinity.

In the case of Saunders Rd, no suitable features exist in the vicinity for indirect monitoring. The section of Saunders Rd which was rendered unstable due to culvert washout runs through a forested area of Cornwall. Features along this road, such as street signs and transmission poles, that maintain interferometric coherence in other areas of Cornwall and that could potentially be used to infer deformation of this road are obscured by the foliage immediately adjacent to it on either side. In contrast, the intersection of Emma Ave and Cumberland St is relatively free of vegetation, and buildings are far enough removed such that the intersection itself is not concealed by radar shadow. Figure 35 shows that the ends of the corroded culvert are uncovered and 
were able to be monitored with InSAR. This figure also shows that the dimensions of the exposed ends of the culvert are not much greater than the pixel spacing, such that the response from the culvert consists of only a small cluster of pixels. This demonstrates that care must then be taken, in iteration 1 stage 2 , to select multilook processing ratios that do not decrease the geometrical resolution to such an extent that smallest potential feature of interest would be obscured. Prior to the selection of multilook processing ratios, all features for which monitoring is desired should be considered: if there is potential that direct monitoring would not be possible, but indirect monitoring via adjacent features may be, then multilook processing ratios should be selected on the basis of maintaining resolution of the smallest potential feature of interest. In this project, a 1:3 and 1:2 ratio were used for the SLA74 and SLA24 datasets, respectively. Neither ratio reduces geometrical resolution to the extent that the road surface is obscured; however, relative to the size of the culvert the 1:2 ratio is more suitable. A caveat to this statement is that, as described in the railway line case study, small features with interferometric phase differences that differ significantly from adjacent pixels tend to cause phase unwrapping errors. With greater multilook ratios, this phase noise is reduced, making phase unwrapping easier. In the SLA74 dataset the intersection of Emma Ave and Cumberland St contained significant noise and phase unwrapping errors, such that the pixels representing the culvert had to be removed from the phase unwrapping mask. 


\subsubsection{R. H. Saunders Generation Station Access Road}

The access road to the R. H. Saunders Generating station is built on a raised embankment with one side sloping towards the St Lawrence River, and the other towards the city of Cornwall. As with Saunders Rd and the Emma Ave and Cumberland St intersection, the road surface itself is incoherent. In this case, indirect monitoring of the road surface via adjacent features is straightforward since the embankment is constructed from relatively coarse gravel, and as such strongly backscatters the radar signal resulting in very high interferometric coherence. Figures 34 and 37 demonstrate that this coherence is somewhat a function of road orientation. For the SLA74 deformation map the river-side of the embankment is continuously coherent, with some reduction of coherence along the east-west segment. In contrast the city-side of the embankment is not detected (with suitable coherence), except for the small segment with a northwestern aspect. Similarly, for the SLA24 deformation map, the river-side of the embankment is continuously coherent, but the coherent pixel density is greatest for sections with a southeastern aspect. Likewise, the city-side of the embankment is only monitored for segments with a predominantly eastern aspect. The variation in coherence as a function of road orientation is again the result of acquisition geometry. The SLA24 dataset was acquired in a right-looking, descending geometry. Slopes with a predominantly eastern aspect are fully illuminated by the radar beam, whereas slopes with a western aspect are eclipsed somewhat. The opposite is true for the SLA74 dataset, which is also right-looking but acquired in an ascending orbit. 


\subsubsection{Major Contributions to Error}

During the examination of the case studies, phase unwrapping errors were identified as a major contribution to error, especially for (but not limited to) the railway line case study. Other factors, namely atmospheric effects and the size of the datasets also contribute to error. Prior to elaborating on these factors, the effect of acquisition geometry on the noise floor is first discussed below.

Figures 27 and 28 show the average noise level of each time series deformation map, and the average error level of each stable reference point, respectively. From these figures it is clear that the error level of the SLA24 dataset is generally higher than that of the SLA74 dataset, despite the fact that the SLA24 dataset is larger. The primary reason for this is that the incidence angle of the SLA24 dataset (nominally $47.25^{\circ}$ at the image center) is larger than that of the SLA74 dataset (nominally $24.75^{\circ}$ at the image center). With the InSAR technique, deformation is measured in the LOS direction (i.e. towards or away from the center). If interferograms from only one orbit (i.e. ascending

or descending) are available, as in this project, vertical deformation (subsidence) can be calculated only if it is reasonable to assume that no other component of the deformation is significant. In that case, the LOS deformation can simply be projected onto the vertical plane since the incidence angle, $\theta$, is known (as shown schematically in Figure 47). Likewise, with the InSAR technique, the error on measured deformation is also in the LOS direction and therefore the vertical component of the LOS error is the projection of the LOS error onto the vertical plane, such that the error on the vertical 
component of deformation ( $\left.\sigma_{\text {vertical }}\right)$ is greater than that of the error on the LOS

deformation ( $\left.\sigma_{\mathrm{LOS}}\right)$ :

$$
\sigma_{\text {vertical }}=\frac{\sigma_{L O S}}{\cos \varphi}
$$

The percent increase in the error on vertical deformation as a result of increasing the incidence angle from that of SLA74 to that of SLA24 is:

$$
\begin{gathered}
\% \text { Increase }=\frac{\sigma_{\text {Vertical,SLA24 }}-\sigma_{\text {Vertical }, S L A 74}}{\sigma_{\text {Vertical,SLA74 }}} \times 100 \% \\
\% \text { Increase }=\frac{\frac{\sigma_{L O S, S L A 24}}{\cos \varphi_{S L A 24}}-\frac{\sigma_{L O S, S L A 74}}{\cos \varphi_{S L A 74}}}{\frac{\sigma_{L O S, S L A 74}}{\cos \varphi_{S L A 74}}} \times 100 \%
\end{gathered}
$$

Which, if $\sigma_{L O S, S L A 74}=\sigma_{L O S, S L A 24}$, is simply:

$$
\begin{gathered}
\% \text { Increase }=\frac{\frac{1}{\cos 47.25^{\circ}}-\frac{1}{\cos 24.75^{\circ}}}{\frac{1}{\cos 47.25^{\circ}}} \times 100 \% \\
\% \text { Increase }=25.25 \%
\end{gathered}
$$

Therefore, on the basis of incidence angle alone, the error on vertical deformation of the SLA24 dataset is approximately $25 \%$ greater than for the SLA74 dataset. 


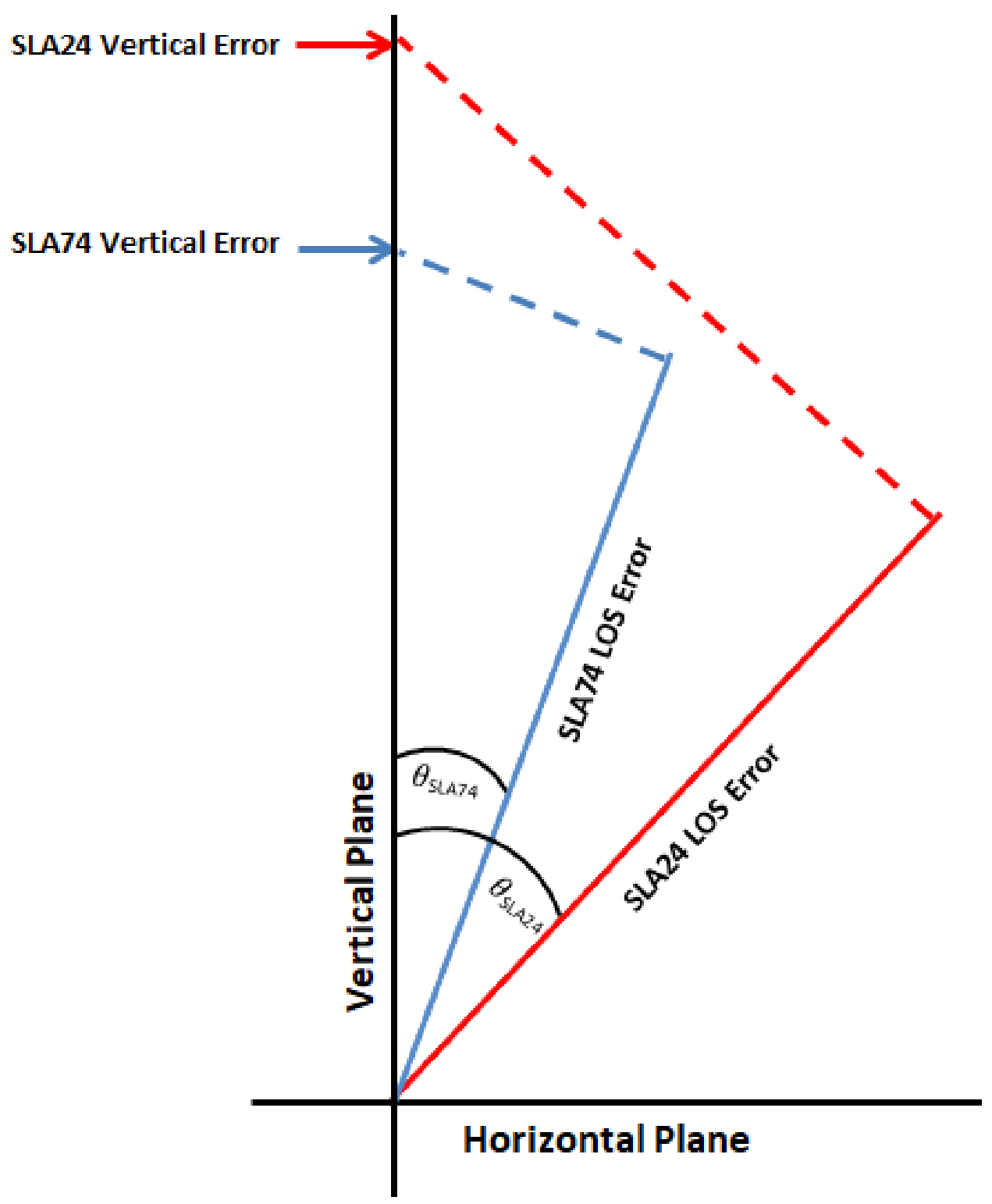

Figure 47: Diagram showing the projection of SLA74 and SLA24 LOS errors of equal magnitude onto the vertical plane. 
In addition to phase unwrapping errors and the innate error due to incidence angle, atmospheric effects and the limited size of the datasets used also contribute to error. When selecting interferograms to be input into the multi-baseline algorithm (Section 5.1.2.), careful attention was paid to select interferograms that displayed only minimal atmospheric effects. However, many interferograms still contained notable atmospheric phase delay, even where weather records indicated clear conditions. During the interferogram selection process it was also noted that the phase trends attributed to atmospheric path delay tended to be more prominent close to the St. Lawrence River, with the R.H. Saunders Generating Stations access road appearing to be particularly affected. Additionally, the SLA74 dataset seemed to have more prominent atmosphere related phase trends than the SLA24 dataset. The SLA74 dataset was acquired at about 22:42 (UTC) i.e. 5:42 or 6:42 PM (EST) (depending on daylight savings time), and the SLA24 dataset was acquired at 10:57 (UTC), i.e. 5:57 or 6:57 AM (EST). The SLA74 images were acquired in the evening, after the Saint Lawrence River had been subject to the heat of the sun for a full day. These observations suggest that the phase delay resulting from low altitude water vapour and humidity coming off the dam reservoir and river, respectively may be present in interferograms in a similar way to atmospheric path delay, although of a lower magnitude.

The simplest way to reduce error in the deformation maps would be to increase the number of images used in the analysis. The multi-baseline analysis computes the time-series deformation from a set of multi-master interferograms by implementing a weighted least-squares algorithm. To minimize the contribution of error present in 
individual interferograms, the interferogram set should be overspecified, i.e. more than one (ideally numerous) interferograms should contain information from each SLC such that these observations can be correlated. The result of removing so many interferograms from the analysis because of phase unwrapping errors and atmospheric effects is that the interferogram set is not overspecified for some time intervals (Figure 22). Increasing the number of SLC images used in the analysis allows for a more restrictive selection of interferograms on the basis of phase unwrapping errors and atmospheric effects, while also increasing the degree of overspecification of the interferogram set. 


\subsection{Sentinel-1}

In the Sentinel-1 dataset, a component of the IPD that appears to be uncorrelated to ground deformation remains after the baseline refinement. The magnitude and pervasiveness of this unidentified component of the interferometric phase difference prevented the application of the multi-baseline algorithm to this dataset. In section 7.2.1., the characteristics of this phase component are discussed. In section 7.2.2., the potential of the Sentinel-1 dataset for infrastructure monitoring, in terms of image resolution and coherence, is discussed.

\subsubsection{Constraining IPD components}

The unwrapped, differential interferograms presented in Section 6.2 were generated using the GAMMA software. The topographic phase component was removed by using a DSM to simulate the interferometric phase difference resulting from the topography. Consequently, two potential sources of error are present in this processing sequence:

1) An incorrect implementation of the GAMMA software. As described in Sections 4.5.2. and 5.2.2., the TOPSAR acquisition mode of the Sentinel-1 dataset required that the processing sequence applied to the RADARSAT- 2 dataset be adapted somewhat. In doing so, the possibility of incorrectly incorporating GAMMAs various TOPSAR-specific algorithms within the processing framework established for the RADARSAT-2 dataset arises.

2) Incomplete or improper removal of the topographic phase. Removing the topographic phase involved first importing a DSM into the GAMMA software, 
subsequently clipping this DSM to the same extent as the Sentinel-1 data and transforming it into RDC, and finally simulating and subtracting the topographic phase from the interferogram. Inaccuracies in this process can be introduced in a variety of ways, such as specifying an incorrect datum or projection of the DSM.

The effect of these two potential sources of error is easily verified by using the SNAP software. This toolbox was developed by the ESA specifically to support the exploitation of ERS, ENVISAT, Sentinel-1/2/3, and some third party (including RADARSAT-2) data (ESA 2017e). In its current form, SNAP provides TOPSAR-specific methods for coregistration, interferogram calculation, and topographic phase removal, but not for phase unwrapping. Using the SNAP software, two interferograms, one with and one without topographic phase removal, were created from the VV polarized acquisitions 2015-0908 and 2015-09-20. In Section 6.2., the unwrapped interferogram created from the same data using the GAMMA software was presented, and noted to contain significant phase noise. Figures 48 and 49 compare the IW1 subswath in the 2015-09-08 to 201509-20 interferograms, prior to topographic phase removal, generated by the GAMMA software and the SNAP software, respectively. The interferogram in Figure 48 is from Iteration 1 stage 2 (i.e. the first suite of interferograms, prior to baseline refinement) for consistency with the interferogram in Figure 49. Both interferograms were similarly filtered to reduce phase noise; Figure 48 with GAMMAs adaptive spectral filtering algorithm and Figure 49 with SNAP's Goldstein phase filtering algorithm. 
Likewise, Figures 50 and 51 compares the IW1 subswath region in the 2015-09-08 to 2015-09-20 interferograms, after topographic phase removal, generated by the GAMMA software and the SNAP software, both with the same filtering applied to Figure 48 and 49. The same DSM (Section 4.5.3.) was used to remove the topographic phase in both examples.

Figure 48 and 49 shows identical phase trends, with slightly different magnitudes being attributed to different filtering and radiometric calibration between the two softwares. Similarly, Figure 50 and 51 show nearly identical phase trends, indicating that during the processing sequence described in Section 5.2. the topographic phase was successfully removed. The consistency between interferograms generated with GAMMA, as described in Section 5.2., and interferograms generated using the SNAP TOPSAR-specific processing sequence suggests that phase trends present in the filtered, unwrapped, baseline refined interferograms is not due to an improper implementation of the GAMMA software. 


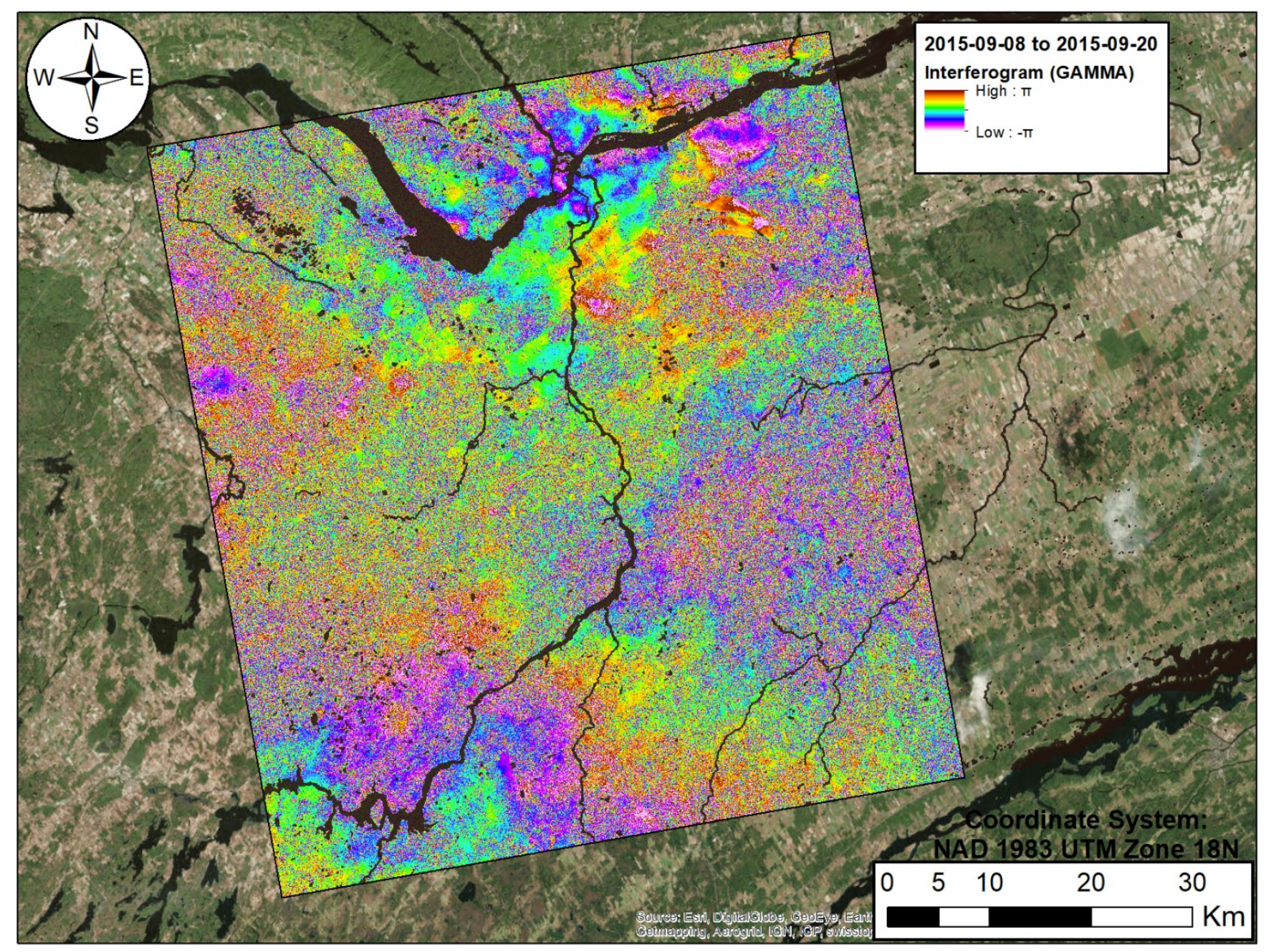

Figure 48: Interferogram without topographic phase removal created with the GAMMA software from IW1 of 2015-09-08 and 2015-0920. The interferogram is presented at $6 \pi$ per colour cycle. 


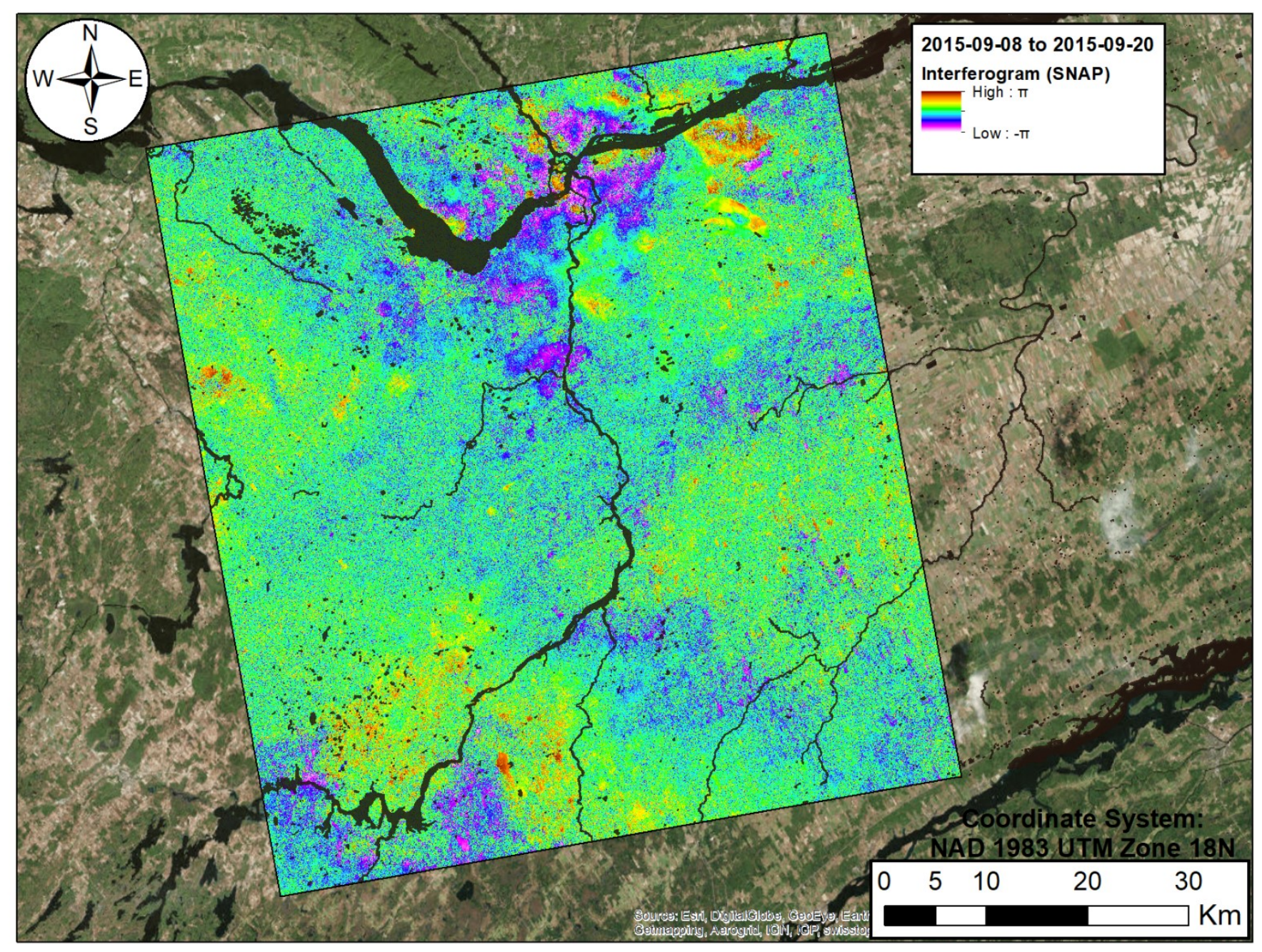

Figure 49: Interferogram without topographic phase removal created with the SNAP software from IW1 of 2015-09-08 and 2015-09-20. The interferogram is presented at $6 \pi$ per colour cycle. 


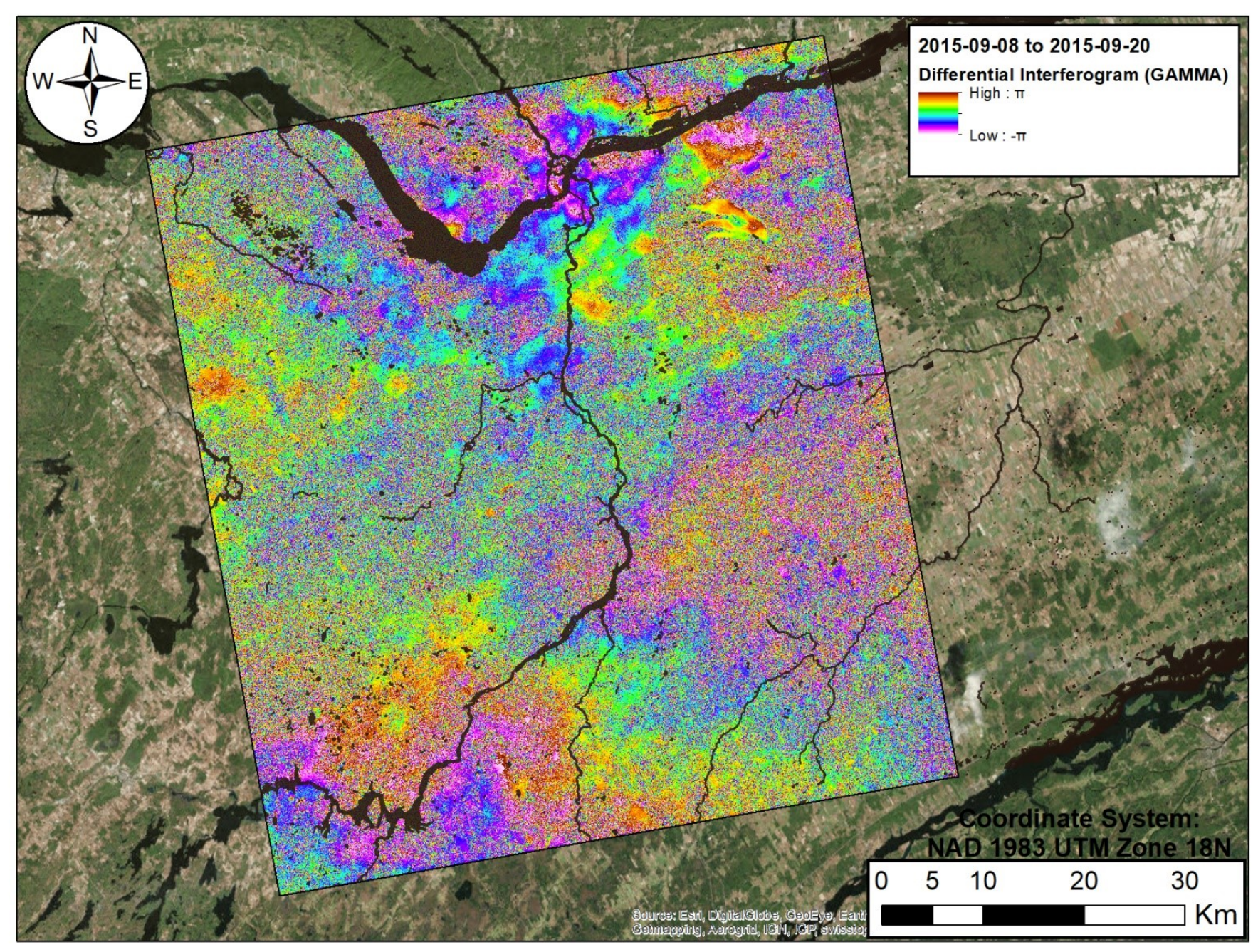

Figure 50: Interferogram with topographic phase removal created with the GAMMA software from IW1 of 2015-09-08 and 2015-09-20. 


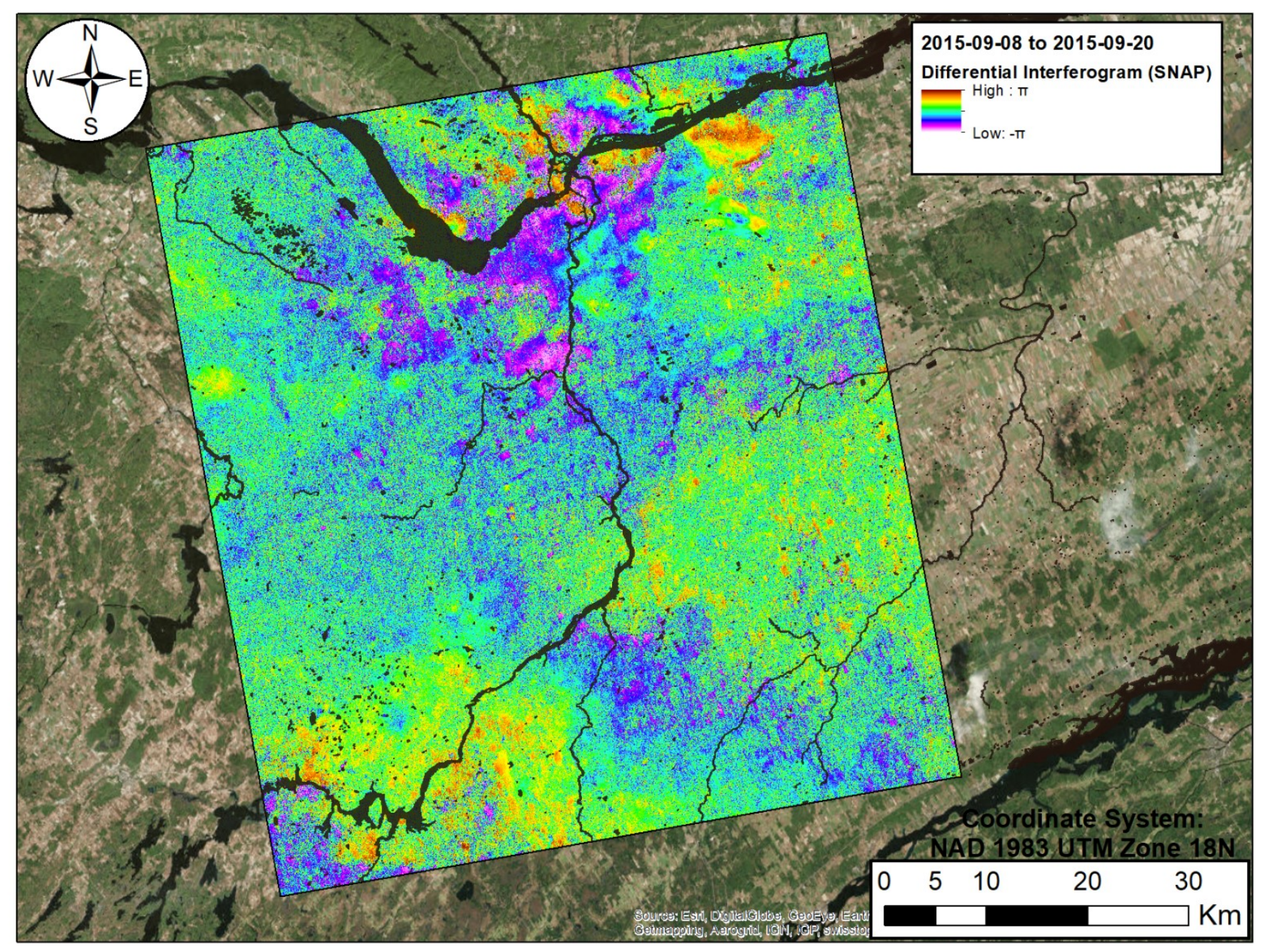

Figure 51: Interferogram with topographic phase removal created with the SNAP software from IW1 of 2015-09-08 and 2015-09-20. 
To see the effect of polarization on the residual phase trend the same interferogram as in Figure 49 was made, with the SNAP software, from the VH polarized component of the 2015-09-08 and 2015-09-20 acquisitions. This interferogram is shown in Figure 52. The phase trends of the VH polarized interferogram are clearly identical to the VV polarized interferogram. The coherence, however, of the $\mathrm{VH}$ polarized interferogram is slightly reduced, notably along linear infrastructure elements, for e.g. highway 417 east of Kanata. 


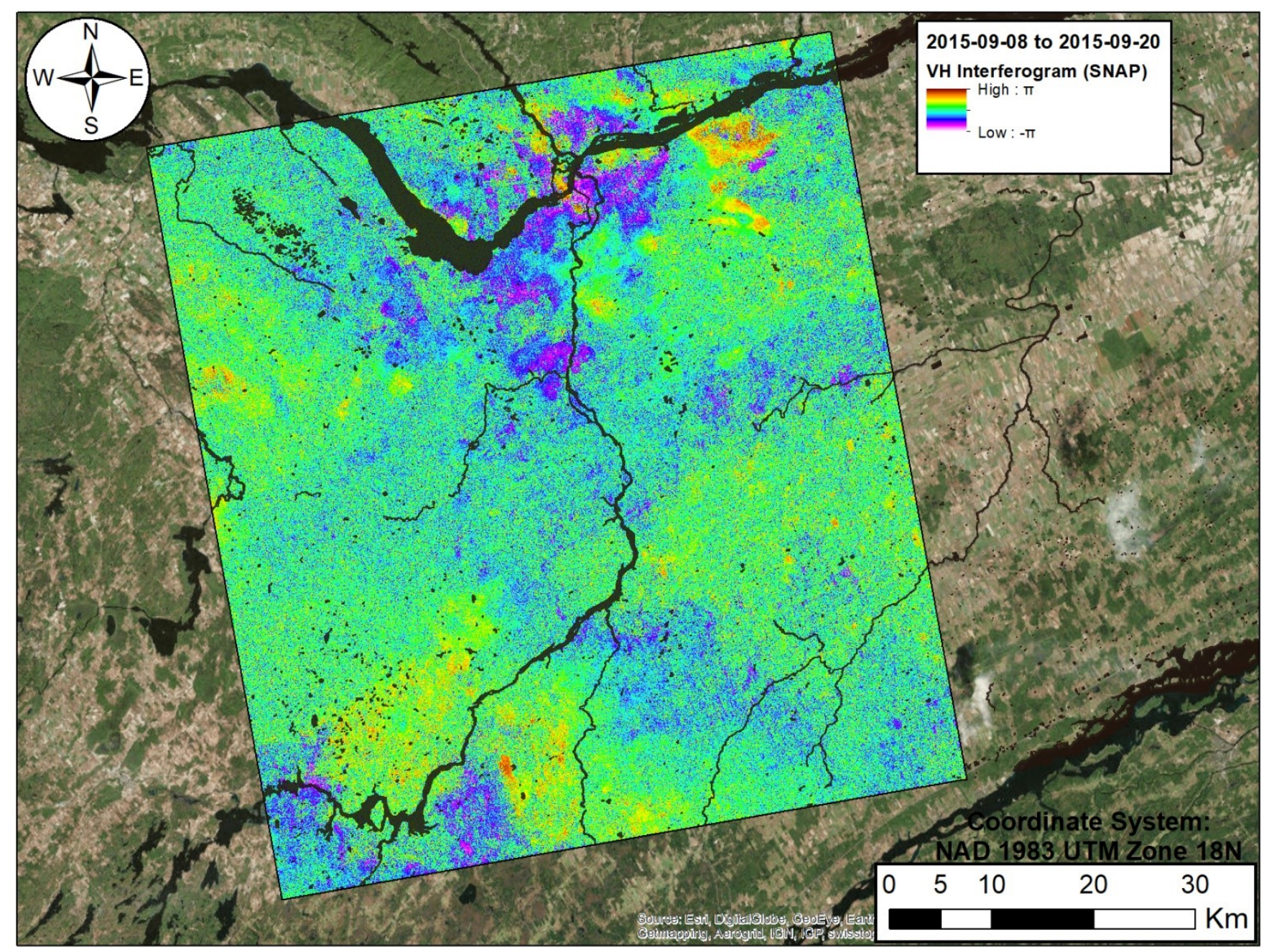

Figure 52: Interferogram without topographic phase removal created with the SNAP software from the VH polarized component of IW1 of 2015-09-08 and 2015-09-20. 
In the above discussion, improper implementation of the GAMMA software and incorrect or incomplete removal of the topographic phase component were ruled out as potential sources of the remaining interferometric phase components. Significant precipitation events can also be ruled out. According to the weather radar images shown in Figure 39, at 22:50 (UTC) on 2015-09-08 some precipitation was occurring north of Ottawa but nowhere else in the study area. Weather reports (Table 2 ) indicate, however, that no precipitation fell in Ottawa on that day. On 2015-09-20 no precipitation was occurring anywhere in the study area. The region corresponding to the precipitation on $2015-09-08$ is indicated by the red box in Figure 38. Within this region, areas of low interferometric phase can be attributed to atmospheric path delay. Figure 38 shows, however, that the areas with the highest interferometric phase are outside of this area. The weather radar image of Figure 39 only shows precipitation, not total cloud cover. It is possible that cloud cover occurs throughout the study area, but the atmospheric path delay caused by non-precipitating clouds is expected to be less than that of precipitating clouds. In Appendix E, weather radar images taken at (approximately) the same time as of each Sentinel-1 acquisitions used in the interferograms shown in Sections 6.2. and Appendix C are presented. Comparing these weather radar images to the interferograms presented in Section 6 and Appendix C, it is clear that interferograms made with acquisitions that are clear of precipitation still contain the unidentified IPD component. The weather radar image of 2015-11-19 shows precipitation occurring at the time of Sentinel-1 acquisition, and this precipitation is clearly seen as a large region of high interferometric phase in interferogram 2015-11- 
19 to 2015-12-13 (Figure 60, Appendix C). Interferogram 2016-03-30 to 2016-04-23

(Figure 42) shows a region of high interferometric phase of comparable size and magnitude, but no precipitation was falling at the time of acquisitions. Table 2 shows that some precipitation fell earlier in the day on 2016-03-30.

The unidentified IPD component tends to correlate to surficial features (notably the Mer Bleue and Alfred's bogs, and agricultural fields). Figures 40 and 41 (interferogram 2017-01-24 to 2017-02-05) show that both bogs are well defined in the interferogram, with these features displaying significantly higher interferometric phase differences than the immediate surroundings. Cornwall is also well defined in this interferogram, with the interferometric phase difference of the city being markedly less than the surroundings. In Figure 53, below, a close up of interferogram 2016-10-20 to 2016-11-01 (Figure 61, Appendix C) is shown. In this interferogram, the interferometric phase difference is clearly correlated to the presence of agricultural fields. Wetlands and agricultural fields have significantly different surface moisture and vegetation than the surrounding areas. Based on these observations, the unidentified IPD component is putatively attributed to change surface moisture content. Ground reflectivity is a function of surface moisture content, and therefore if surface moisture content changes between SAR acquisitions, the assumption that $\phi_{\rho}$ (in equation [12]) is equal to zero is invalid. 


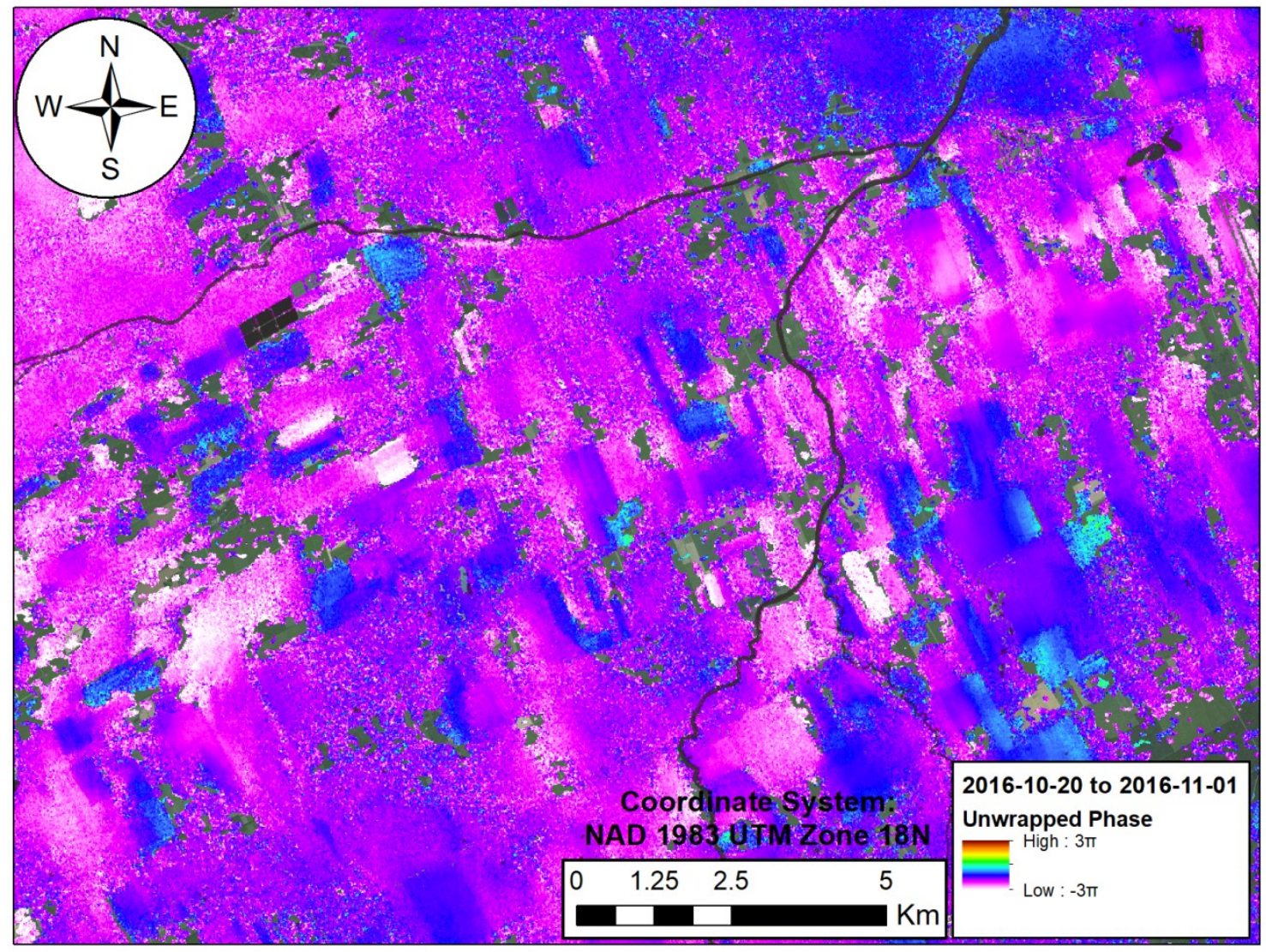

Figure 53: Close up of an agricultural area from the Sentinel-1 interferogram 2016-10-20 to 2016-11-01. 


\subsubsection{Potential for InSAR Monitoring with S1 Images}

In Appendix B, MLI images of the full study area, and of the Cornwall region, are presented. In Appendix D, coherence maps of the full study area, and of the Cornwall region, for each of the interferograms in Section 6 and Appendix $C$ are also presented.

The $\mathrm{CN}$ railway line and the R.H. Saunders generating station access road are clearly well within the resolution of the MLI image and both display high coherence, similarly to the RADARSAT-2 dataset. Direct monitoring of these features, as in the RADARSAT-2 analysis, could be possible with this dataset if the unidentified IPD component could be removed. The intersection of Emma Ave and Cumberland St is within the resolution of the MLI image, however, is of very low intensity. In contrast to the RADARSAT-2 dataset, the culvert or other surrounding features, which could be used to indirectly monitor road deformation are not within the resolution capabilities of this dataset, and as such no monitoring can be performed. Similarly, Saunders Rd is not clearly resolved in the MLI image and cannot be monitored. The St. Johns Presbyterian Church is difficult to discern in the $\mathrm{MLI}$ image since its dimensions are comparable to those of the ground resolution cell, indicating that the resolution of this dataset is better suited to monitoring neighbourhood sized trends rather than for identifying settlement (or differential settlement) of individual buildings.

The coarser resolution of the Sentinel-1 dataset compared to the RADARSAT- 2 dataset means that streets and individual buildings will not be able to be monitored unless these features are adjacent to a larger coherent region. This coarser resolution also means that small transient features less affect the phase of each cell, allowing for 
very high coherence and reduced phase unwrapping errors. The interferogram in Figure 44 (its associated coherence map can be found in Appendix D) was created from acquisitions taken almost a year apart (2015-10-26 and 2016-10-08) but the coherence of Ottawa and Cornwall are still both suitable to perform deformation monitoring. The 12 day repeat cycle further increases this coherence to the point where even vegetated areas such as agricultural fields could potentially be monitored; in the RADARSAT-2 dataset no vegetated areas showed this potential. The large swath size and open data policy makes this dataset a potentially valuable asset to geohazard monitoring, particularly if combined with higher resolution dataset. Should the unidentified component of the interferometric phase difference be identified and removed, then this dataset could be used to identify small, specific regions in which deformation is occurring, or likely to occur, and these smaller regions could then be monitored with a higher resolution dataset such as the RADARSAT-2 Spotlight mode. 


\section{Chapter 8: Research Summary and Recommended SAR}

\section{Specifications}

In this research project, SAR interferometry was used to monitor linear infrastructure subject to geohazards in Eastern Ontario. Two study areas were selected for monitoring; the first study area was the City of Cornwall, Ontario, and the second study area was the much larger region that contains Cornwall, but additionally the Ottawa and East Hawkesbury regions of Eastern Ontario. The primary study site was analyzed with two RADARSAT-2 Spotlight mode datasets spanning March 2015 to September 2016: 15 ascending acquisitions with small incidence angles (SLA74) and 19 descending acquisitions with large incidence angles (SLA24) were used to create InSAR derived time-series deformation maps of Cornwall. The secondary study area was analysed by examining interferograms created from 33 Sentinel-1A Interferometric Wide mode acquisitions from 28 dates spanning July 2015 to April 2017.

To create the interferograms and deformation maps an InSAR processing sequence was developed for the Spotlight mode data, and subsequently adapted to process the Sentinel-1 IW acquisitions. This processing sequence maximizes the exploitation of small datasets by using a multi-master interferogram approach. The resultant SLA74 and SLA24 derived deformation maps had noise floors of $\pm 1.5 \mathrm{~cm}$ and $\pm 1.0 \mathrm{~cm}$, respectively. No deformation maps were created from the Sentinel-1A dataset since an IPD component, not related to ground deformation, remained after the processing. 
The current limitations of InSAR for the monitoring of linear transportation taking into account the characteristics of SAR data and using state of the art processing techniques were examined through a variety of case studies in Cornwall, Ontario: a twotrack portion of the $\mathrm{CN}$ railway line, the $\mathrm{R}$. $\mathrm{H}$. Saunders generating stations access road, two sections of city streets experiencing surface failure (Saunders Rd, and the intersection of Emma Ave. and Cumberland St.), and the St. Johns Presbyterian Church. The processing techniques employed in this project allowed for all features, except for roads (which are incoherent in C-band), to be directly monitored with Spotlight mode data. However, ground deformation was not identified or quantified since the noise floor is greater than the expected magnitude of deformation over a time span of less than two years. Phase unwrapping errors, atmospheric path delay, and limited SAR data were identified as making the largest contributions to the noise floor.

\subsection{SAR Dataset Recommendations for Infrastructure Monitoring}

In Chapter 7, image resolution, incidence angle, re-visit period, and size of the dataset were identified as characteristics that determine the suitability of a SAR dataset for infrastructure monitoring. These observations are synthesised into recommendations for the selection of SAR datasets for future projects of a similar nature to the case studies presented in this thesis.

1. Small incidence angle. InSAR deformation measurements (and the associated error) are made in the LOS direction, and subsequently projected onto the vertical plane to assess vertical deformation. To reduce the error on vertical deformation, a small 
incidence should be used. Minimizing the incidence angle also maximizes the intensity of the backscattered signal received by the sensor, and reduces the amount of radar shadow. A corollary to this statement is that layover increases with decreasing incidence angle. In areas with very steep (or vertical) slopes such as buildings, the need for accurate vertical deformations, strong received signal, and reduced radar shadow has to be balanced with image resolution and the potential impacts of layover on a case-by-case basis. For SAR platforms that operate in preprogrammed acquisition plans, such as the current Sentinel-1 mission and upcoming RCM mission, the incidence angle must be suitable for a variety of applications. The incidence angle of Sentinel- $1 \mathrm{~A}\left(32.9^{\circ}\right.$ to $\left.43.1^{\circ}\right)$ is intermediate to the incidence angles of the SLA24 and SLA74 datasets used in this project, and thus achieved a reasonable balance between maximizing backscatter intensity and vertical deformation sensitivity while minimizing layover. A similar incidence angle is recommended for the RCM.

2. Moderate resolution. A fundamental assumption made by most phase unwrapping algorithms is that the phase between neighbouring pixels varies gradually. This assumption holds better for lower resolution than higher resolution datasets. The phase of each pixel is a function of the response of many elementary scatterers, and as such the larger the ground resolution cell the less effect inhomogeneities within the cell have on the received phase. Therefore the phase of neighbouring pixels in a low resolution dataset tend to be more similar than in a high resolution dataset (the phase of which is strongly effected by small transient features), making phase 
unwrapping easier. The moderate resolution of the Sentinel-1 dataset does not allow monitoring of small features such as individual buildings, but does allow for monitoring of linear infrastructure such as railways. A similar resolution, or slightly higher, is recommended for the RCM mission.

3. Large data sets. An overspecified interferogram suite is required in order to apply the multi-baseline algorithm. The greater the degree of overspecification, the lower the impact that errors present in individual interferograms (e.g. phase unwrapping errors, atmospheric path delay, or phase noise) have on the calculated time-series deformation. Furthermore, when deformation rates are low, a strict removal of interferograms affected by atmospheric effects is necessary. Maintaining continuity of the time series while also strictly removing interferograms requires much larger datasets then those used in this project. Open data policies, such as the one currently employed by the ESA, are integral to the feasibility of obtaining suitably large datasets.

4. Small temporal baseline. Coherence of most urban structures is maintained even with relatively long time intervals between acquisitions, particularly in moderate resolution datasets. On the contrary, coherence of agricultural or other vegetated areas degrades quickly, and therefore short time intervals area required.

Furthermore many geohazards occur suddenly, for e.g. mass movements in Leda clay, and can only be detected by the first acquisition after the event. Small temporal baselines improve the usefulness of the monitoring program by maintaining greater coherence through the time series, and allowing for more rapid 
detection of specific events. The revisit period of 12 days for the Sentinel-1A data was suitable for maintaining coherence over the majority of the study area. 


\section{Bibliography}

Abbott, P. L., \& Samson, C. (2012). Natural disasters (2nd Canadian ed.). McGraw-Hill Ryerson, Whitby, Ontario. 464p.

Ai, B., Liu, K., Li, X., \& Li, D. H. (2008). Flat-earth phase removal algorithm improved with frequency information of interferogram. Geoinformatics 2008 and Joint Conference on GIS and Built Environment: Classification of Remote Sensing Images. 71471A-71471A.

Association, C. C., Federation of Canadian Municipalities, \& Canadian Public Works Association. (2012). Canadian infrastructure report card, volume 1: 2012 - municipal roads and water systems. Canadian Infrastructure Report Card. 75p.

Athir, N. (2014). Engineering Characteristics of Sensitive Marine Clays - Examples of Clays in Eastern Canada. (M.A.Sc. Thesis). University of Ottawa, Ottawa, Canada. 205p.

Berardino, P., Fornaro, G., Lanari, R. \& Sansosti, E. (2002). A new algorithm for surface deformation monitoring based on small baseline differential SAR interferograms. IEEE Transactions on Geoscience and Remote Sensing 40 (11), 2375-2383.

Blahut, R. E. (2004). Theory of remote image formation. Cambridge University Press, Cambridge, England. 553p.

Bobrowsky, P. T., \& Dominguez, M. J. (2012). Landslide susceptibility map of Canada Natural Resources Canada. Accessed from:

http://geoscan.nrcan.gc.ca/starweb/geoscan/servlet.starweb?path=geoscan/fulle.we b\&search1=R=291902

Canadian Space Agency. (2017-03-30). RADARSAT Constellation Mission. Accessed from: http://www.asc-csa.gc.ca/eng/satellites/radarsat

Canadian Space Agency (2014-03-21). RADARSAT-1. Accessed from: http://www.asccsa.gc.ca/eng/satellites/radarsat1/

Canadian Space Agency (2015-03-25). RADARSAT-2. Accessed from: http://www.asccsa.gc.ca/eng/satellites/radarsat2

Casu, F. (2009). The Small Baseline Subset technique: A performance assessment and new developments for surface deformation analysis of very extended areas. (PhD thesis). University of Cagliari, CA, Italy. 
Chen, C. W., \& Zebker, H. A. (2000). Network approaches to two-dimensional phase unwrapping: Intractability and two new algorithms. Journal of the Optical Society of America A, 17(3), 401-414.

Crosetto, M., Monserrat, O., Cuevas-González, M., Devanthéry, N., \& Crippa, B. (2016). Persistent scatterer interferometry: A review. ISPRS Journal of Photogrammetry and Remote Sensing 115, 78-89.

Cuervo, V., \& Guthrie, R. (2015). Geohazards and Risk: a Proponent's Guide to Linear Infrastructure. Yukon Environmental and Socio-economic Assessment Board. 50p.

Cumming, I. G., \& Wong, F. H. (2005). Digital processing of synthetic aperture radar data: Algorithms and implementation. Artech House. Boston. 660p.

Curlander, J. C., \& McDonough, R. N. (1991). Synthetic aperture radar: Systems and signal processing. John Wiley and Sons. 672p.

de Vries, F.P.Ph. (1998). Speckle reduction in SAR imagery by various multi-look techniques. Star 36. 49p.

Doubre, C. (2013). Traditional Differential Interferometry Synthetic-Aperture Radar (DInSAR). Ecole et Observatoire des Sciences de la Terre. Accessed from: http://omiv2.ustrasbg.fr/imagemining/documents/IMAGEMINING-Doubre.pdf

European Space Agency. (2017a). European Space Agency's Operational Earth Observation Missions: Envisat. Accessed from:

https://earth.esa.int/web/guest/missions/esa-operational-eo-missions/envisat

European Space Agency. (2017b). European Space Agency's Operational Earth Observation Missions: ERS. Accessed from: https://earth.esa.int/web/guest/missions/esa-operational-eo-missions/ers

European Space Agency. (2017c). European Space Agency's Operational Earth Observation Missions: Sentinel-1. Accessed from: https://earth.esa.int/web/guest/missions/esa-operational-eo-missions/sentinel-1

European Space Agency. (2017d). Radar Course. Accessed from: https://earth.esa.int/web/guest/missions/esa-operational-eomissions/ers/instruments/sar/applications/radar-courses

European Space Agency. (2017e). Scientific Toolbox Exploitation Platform. Software. Accessed from: http://step.esa.int/main/

Ferretti, A., Monti-Guarnieri, A., Prati, C., Rocca, F., Massonnet, D., \& Lichtenegger, J. 
(2007a). InSAR Principles: Guidelines for SAR Interferometry Processing and Interpretation Pt-A. ESA Publications, Nordwijkm, The Netherlands. 115p.

Ferretti, A., Monti-Guarnieri, A., Prati, C., Rocca, F., Massonnet, D., \& Lichtenegger, J. (2007b). InSAR Principles: Guidelines for SAR Interferometry Processing and Interpretation Pt-B. ESA Publications, Nordwijkm, The Netherlands. 71p.

Ferretti, A., Monti-Guarnieri, A., Prati, C., Rocca, F., Massonnet, D., \& Lichtenegger, J. (2007c). InSAR Principles: Guidelines for SAR Interferometry Processing and Interpretation Pt-C. ESA Publications, Nordwijkm, The Netherlands. 40p.

Ferretti, A., Prati, C. \& Rocca, F. (2000). Nonlinear subsidence rate estimation using permanent scatterers in differential SAR interferometry. IEEE Transactions on Geoscience and Remote Sensing 38 (5), 2202-2212.

Ferretti, A., Prati, C. \& Rocca, F. (2001). Permanent scatterers in SAR interferometry. IEEE Transactions on Geoscience and Remote Sensing 39 (1), 8-20.

Gabriel, A. K., Goldstein, R. M., \& Zebker, H. A. (1989). Mapping small elevation changes over large areas - differential radar interferometry. Journal of Geophysical Research, 94(B7), 9183.

Gadd, N. R. (1986). Lithofacies of Leda clay in the Ottawa basin of the Champlain Sea. Geological Survey of Canada 85 (21).

Gall, Q. (2010). The Ottawa-Gatineau Geoheritage Project: Geology of the Ottawa Area. Accessed from:

https://static1.squarespace.com/static/525bf175e4b00f431dc971c0/t/525ca7f6e4b0 48ac9edb6cb0/1381804022438/Geology+Of+The+Ottawa+Area.pdf

Gatelli, F., Monti Guamieri, A., Parizzi, F., Pasquali, P., Prati, C., \& Rocca, F. (1994). The wavenumber shift in SAR interferometry. IEEE Transactions on Geoscience and Remote Sensing, 32(4), 855-865.

Gamma Remote Sensing. (2007). Interferometric SAR Processing. Reference manual. GAMMA Remote Sensing, AG, Worbstrasse 225, CH-3073 Gumigen, Switzerland. 28p.

Gamma Remote Sensing. (2008a). SAR Basics. Reference manual. GAMMA Remote Sensing, AG, Worbstrasse 225, CH-3073 Gumigen, Switzerland. 20p.

Gamma Remote Sensing. (2008b). InSAR Basics. Reference manual. GAMMA Remote Sensing, AG, Worbstrasse 225, CH-3073 Gumigen, Switzerland. 37p.

Gamma Remote Sensing. (2015a). Differential Interferometry and Geocoding 
Software. Reference manual. GAMMA Remote Sensing, AG, Worbstrasse 225, CH3073 Gumigen, Switzerland. 54p.

Gamma Remote Sensing. (2015b). Interferometric SAR Processor -ISP. Reference manual. GAMMA Remote Sensing, AG, Worbstrasse 225, CH-3073 Gumigen, Switzerland. 84p.

Gamma Remote Sensing. (2015c). Interferometric Point Target Analysis Software (IPTA): Users Guide. Reference manual. GAMMA Remote Sensing, AG, Worbstrasse 225, $\mathrm{CH}-3073$ Gumigen, Switzerland. 84p.

Gamma Remote Sensing. (2015d). Sentinel-1 processing with GAMMA Software. Reference manual. GAMMA Remote Sensing, AG, Worbstrasse 225, CH-3073 Gumigen, Switzerland. 39p.

Ghiglia, D. C. \& Pritt, M. D. (1998). Two-dimensional phase unwrapping: Theory, algorithms, and software. John Wiley and Sons. 512p.

Goldstein, R. M., \& Werner, C. L. (1998). Radar interferogram filtering for geophysical applications. Geophysical research letters 25(21), 4035-4038.

Goldstein, R. M., Zebker, H. A., \& Werner, C. L. (1988). Satellite radar interferometry: Two-dimensional phase unwrapping. Radio Science, 23(4), 713p.

H\&P Advanced Technology. (2017). Use of radar technology in a millimeter wave imaging system. Accessed from: http://www.hp-adtec.de/pages/en/projects/radartechnology-terahertz-technology.php?lang=EN

Hanssen, R. F. (2001). Radar interferometry: Data interpretation and error analysis. Kluwer Academic. Dordrecht, London. 298p.

Henschel, M. D., Dudley, J., Lehrbass, B., Sato, S., \& Stöckel, B. M. (2015). Monitoring slope movements from space with Robust Accuracy Assessment. Proc. Slope Stability. p. 12-14.

Hewitt, R.G. (2005). Trees and foundations strategy in areas of sensitive marine clay in the city of Ottawa. Planning and Environment Committee. 78p.

Karrow, P. F. (1965). The Champlain Sea and its Sediments. Soils in Canada: Geological, Pedological, and Engineering Studies. Edited by R. F. Legget. University of Toronto Press. p. 97-108.

Keydel, W. (2007). Normal and differential SAR interferometry. German Aerospace Center (DLR) Wessling (Germany) Microwaves and Radar Inst. 40p. 
Kourkouli, P., Strozzi, T., \& Wegmüller, U. (2012). Comparison of DInSAR and persistent scatterer interferometry for ground-motion monitoring in the Venice lagoon. IEEE 2012 International, 3919-3922.

Lanari, R., Casu, F., Manzo, M., Zeni, G., Berardino, P., Manunta, M., \& Pepe, A. (2007). An overview of the small baseline subset algorithm: A DInSAR technique for surface deformation analysis. Pure and Applied Geophysics 164 (4), 637-661.

Massonnet, D., Feigl, K., Rossi, M. \& Adragna, F. (1994). Radar interferometric mapping of deformation in the year after the Landers earthquake. Nature 369, 227230.

Massonnet, D., Rossi, M., Carmona, C., Adragna, F., Peltzer, G., Feigl, K., \& Rabaute, T. (1993). The displacement field of the landers earthquake mapped by radar interferometry. Nature 364(6433), 138-142.

Massonnet, D., Vadon, H., \& Rossi, M. (1996). Reduction of the need for phase unwrapping in radar interferometry. IEEE Transactions on Geoscience and Remote Sensing 34(2), 489-497.

McCardle, A., McCardle, J., \& Ramos, F. L. G. (2009). Large Scale Deformation Monitoring and Atmospheric Removal in Mexico City. Fringe Workshop 2009.

MacDonald, Dettwiler and Associates. (2016a). RADARSAT-2 Product Description. MacDonald, Dettwiler and Associates Itd, Richmond, BC, Canada. 91p.

MacDonald, Dettwiler and Associates. (2016b). RADARSAT-2 Pricing Information. MacDonald, Dettwiler and Associates Itd, Richmond, BC, Canada. 4p.

Morgan, J., Falorni, G., Bohane, A., \& Novali, F. (2011). Advanced InSAR technology (SqueeSARtm) for monitoring movement of landslides. Central Federal Lands Highway Division, USDOT (Publication No. FHWA-CFL/TD-11-005). 80p.

Morton, S. R. (2015). Development and Evaluation of an Aquifer Capability Screening Tool, Pilot Study: Clarence-Rockland, Ontario. (MSc thesis). Ottawa University, Ottawa, Ontario, Canada.

Nargess, M. \& Rafael, R. (2013) "NASA Computational Case Study: SAR Data Processing Ground-Range Projection". Computing in Science \& Engineering vol. 15. p. 92-95.

National Geophysical Data Center. (1993). Earthquake damage, the landers and big bear earthquakes, June 28, 1992. University of California, California, USA. 6p. 
Natural Resources Canada. (November 20 2015). Radar Image Distortions. Accessed from: http://www.nrcan.gc.ca/node/9325

Oliver, C., \& Quegan, S. (2004). Understanding synthetic aperture radar images. SciTech Publishing. 479p.

Osmanoğlu, B., Sunar, F., Wdowinski, S., \& Cabral-Cano, E. (2016). Time series analysis of InSAR data: Methods and trends. ISPRS Journal of Photogrammetry and Remote Sensing 115, 90-102.

Pinto, N., Simard, M., \& Dubayah, R. (2012). Using InSAR coherence to map stand age in a boreal forest. Remote Sensing 5(1), 42-56.

Powers, D., Youden, J., English, J., Russel, C., Croshaw, S. \& Hanson, R. (2006). InSAR Applications for Highway Transportation Projects. (Technical Report Number: R-05021-260). C-Core, St. John's, Newfoundland, Canada. 91p.

Pritchard O.G., Hallett S.H., \& Farewell T.S. (2013). Road subsidence in Lincolnshire: Soils and road condition. Infrastructure Transitions Research Consortium. 36p.

Pritchard O.G., Hallett S.H., \& Farewell T.S. (2013). Soil movement in the UK - Impacts on critical infrastructure. Infrastructure Transitions Research Consortium. 74p.

Pritchard O.G., Hallett S.H., \& Farewell T.S. (2015). Soil geohazard mapping for improved asset management of UK local roads. Natural Hazards and Earth System Sciences 15, 2079-2090.

Quinn, P. E. (2009). Large landslides in sensitive clay in eastern canada and the associated hazard and risk to linear infrastructure. (PhD thesis). Queen's University, Kingston, Ontario, Canada.

R. J. Fulton (Ed.), Richard, S. H. (1987). Quaternary geology of the Ottawa region, Ontario and Quebec. Energy, Mines, and Resources Canada.

Raspini, F., Bianchini, S., Moretti, S., Loupasakis, C., Rozos, D., Duro, J., \& Garcia, M. (2016). Advanced interpretation of interferometric SAR data to detect, monitor and model ground subsidence: outcomes from the ESA-GMES Terrafirma project. Natural Hazards 83(1), 155-181.

Richards, M. A. (2007). A beginner's guide to interferometric SAR concepts and signal processing. IEEE Aerospace and Electronic Systems Magazine, 22(9), 5-29.

Rosen, P.A., Hensley, S., Joughin, I.R., Li, F.K., Madsen, S.N., Rodriguez, E. \& Goldstein, 
R.M. (2000). Synthetic aperture radar interferometry. IEEE 2000 (88), 333-382.

Ryerson, R. A., Lewis, A. J., Henderson, F. M., \& American Society for Photogrammetry and Remote Sensing. (1998). Principles and applications of imaging radar (3rd ed.). John Wiley and Sons. 896p.

Scaioni, M., Longoni, L., Melillo, V., \& Papini, M. (2014). Remote sensing for landslide investigations: An overview of recent achievements and perspectives. Remote Sensing, 6(10), 9600-9652.

SkyGeo. (2017). InSAR Technical Background. Accessed from: https://skygeo.com/insar-technical-background/

SkyRadar. (2017). E-learning: Radar basics and synthetic aperture radar. Accessed from: http://radarportal.skyradar.com/e-learning-radar-basics-and-syntheticaperture-radar

Touzi, R., Lopes, A., Bruniquel, J., \& Vachon, P. W. (1999). Coherence estimation for SAR imagery. IEEE Transactions on Geoscience and Remote Sensing, 37(1), 135-149.

UNAVCO. (7 August 2015). Synthetic Aperture Radar (SAR) Satellites. Retrieved from: https://www.unavco.org/instrumentation/geophysical/imaging/sar-satellites/sarsatellites.html

Vanier, D. J. \& Rahman, S. (2004). MIIP Report: A Primer on Municipal Infrastructure Asset Management. National Research Council. 65p.

Vanier, D. J. \& Rahman, S. (2004). MIIP Report: Survey on Municipal Infrastructure Assets. National Research Council. 46p.

Wang, Y., Zhu, X. X., \& Bamler, R. (2012). Retrieval of phase history parameters from distributed scatterers in urban areas using very high resolution SAR data. ISPRS Journal of Photogrammetry and Remote Sensing 73, 89-99.

Wegmüller, U., Werner, C., \& Strozzi, T. (2003). GAMMA Interferometric Point Target Analysis Software (IPTA): Users Guide. Reference manual. GAMMA Remote Sensing, AG, Worbstrasse 225, CH-3073 Gumigen, Switzerland. 30p

Wiesmann, A., Werner, C., Strozzi, T., \& Wegmuller, U. (2004). Combination of point and extended target based interferometric techniques. Proc. IEEE 2004 International 2, 989-991. 


\section{Appendix A: RADARSAT-2 MLI Images}

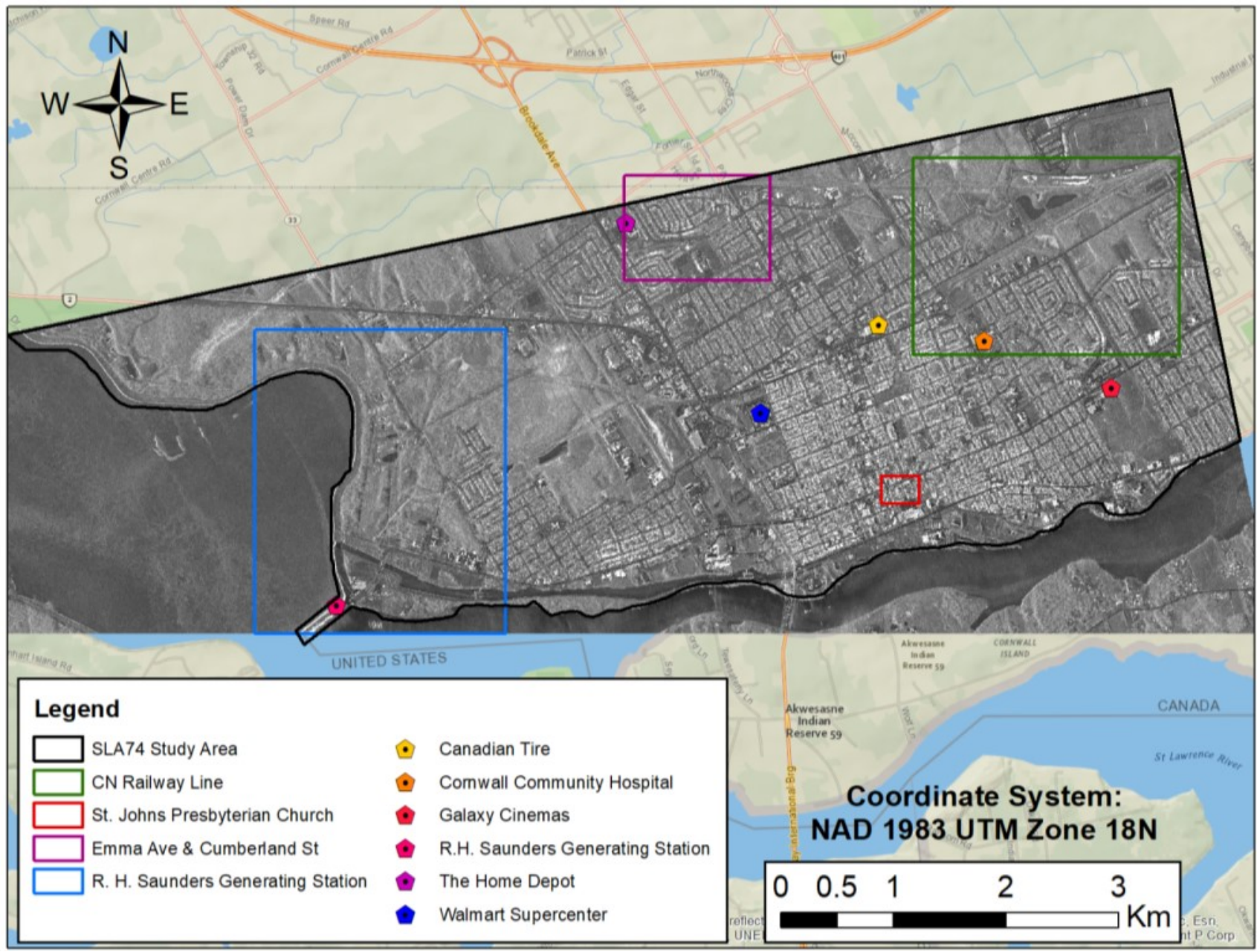

Figure 54: Geocoded MLI Image of the SLA74 study area, case study locations, and reference points. 


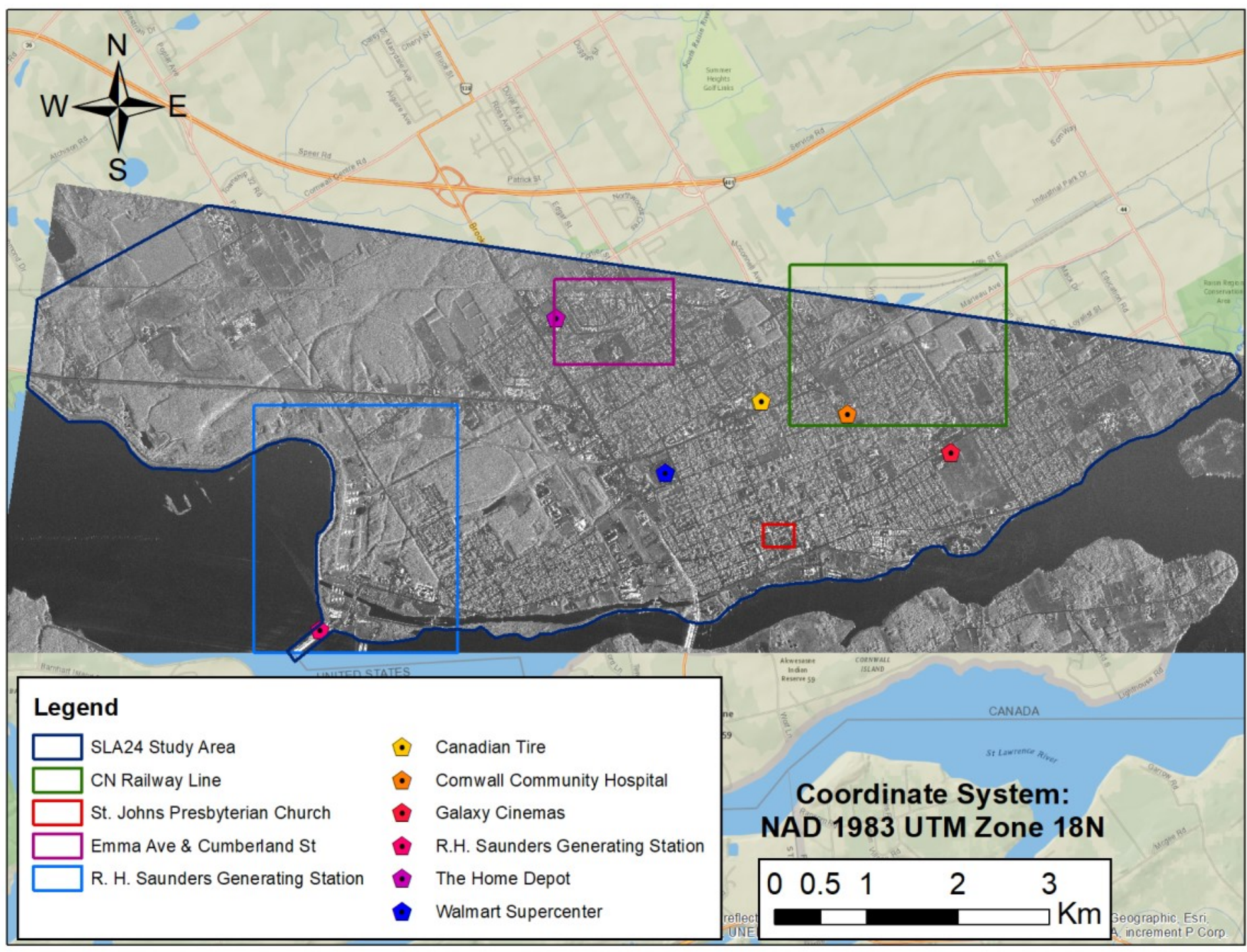

Figure 55: Geocoded MLI Image of the SLA24 study area, case study locations, and reference points. 


\section{Appendix B: Sentinel-1 MLI Images}

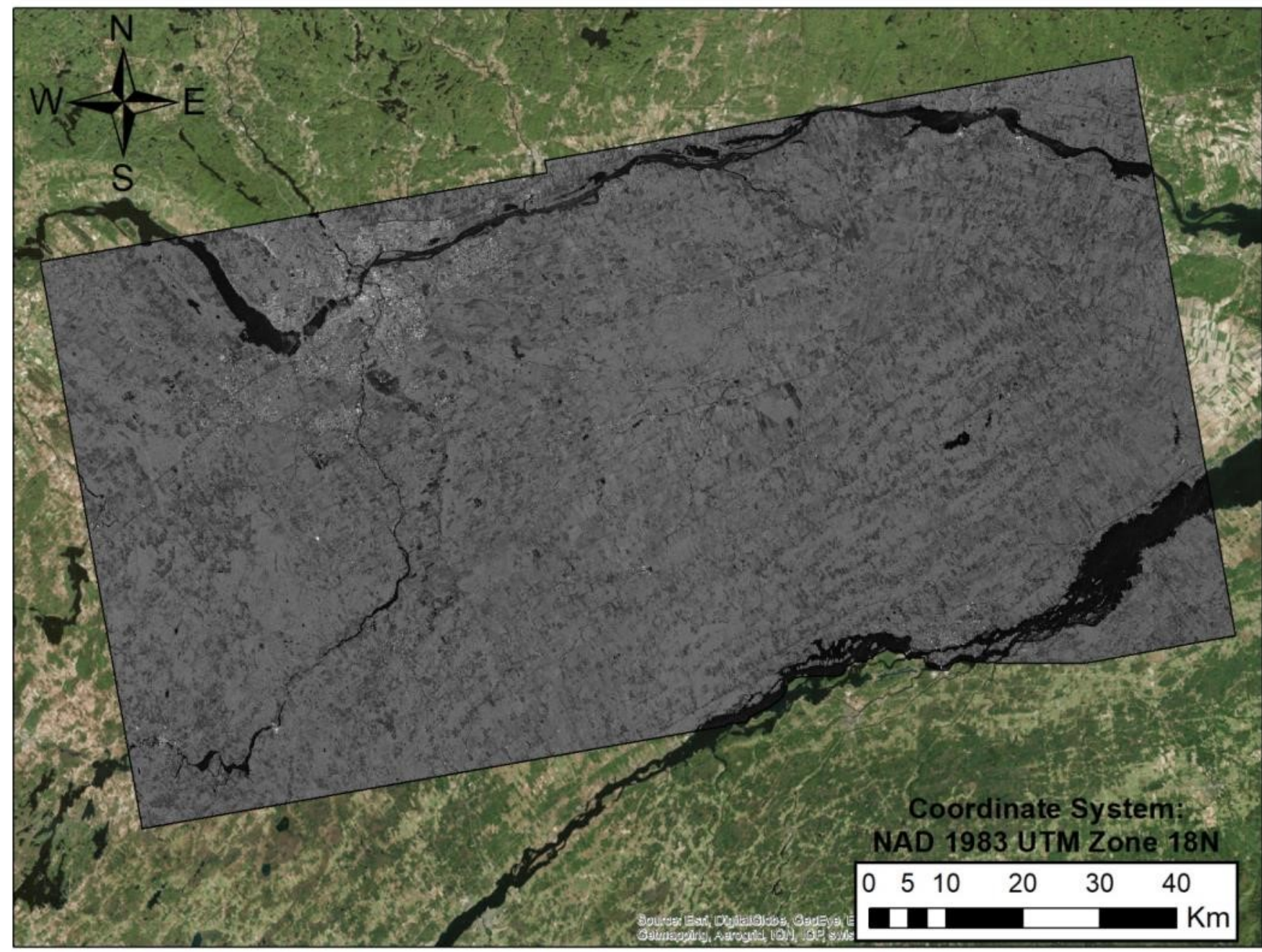

Figure 56: Geocoded MLI image of the Sentinel-1 study area. 


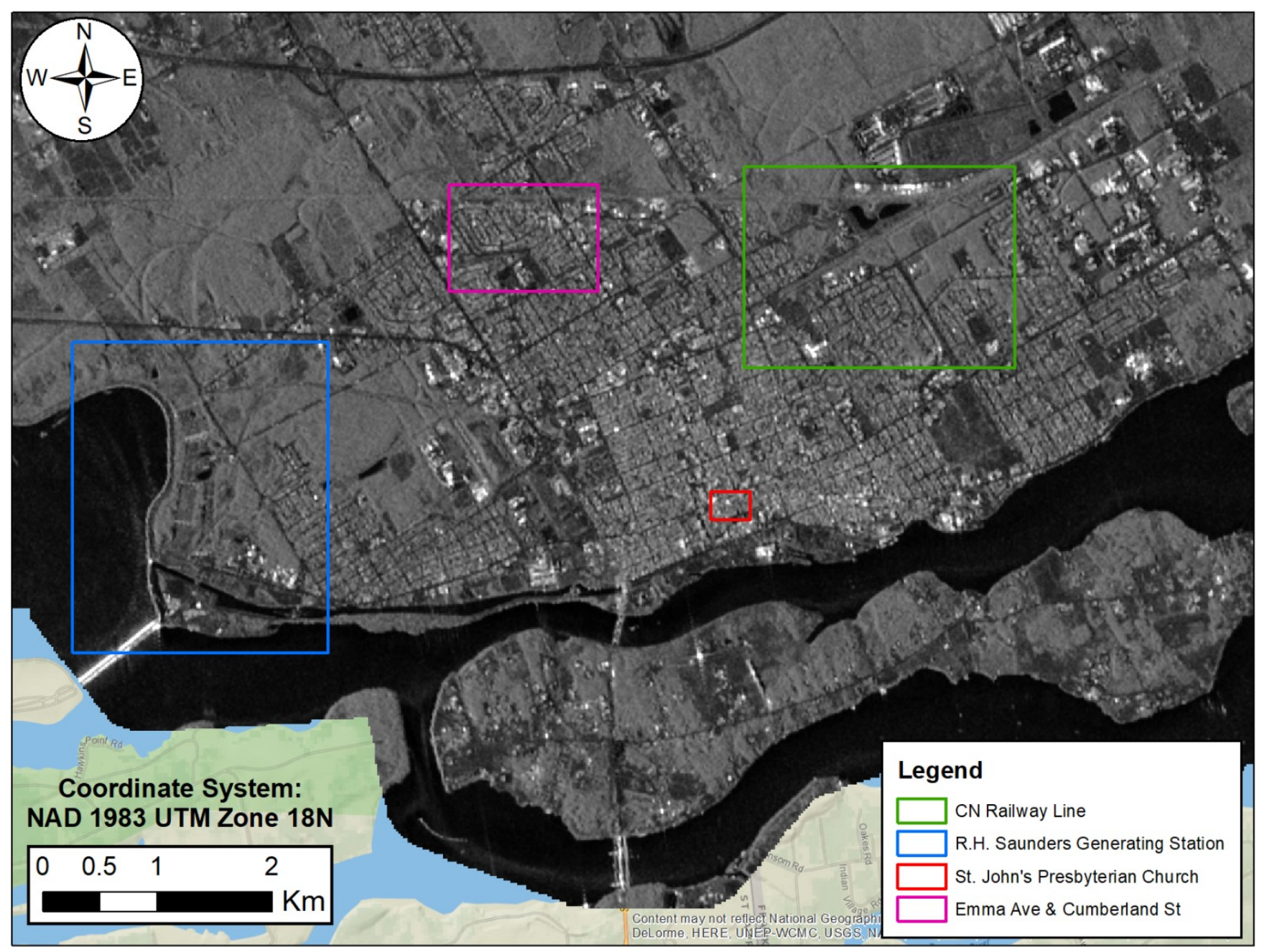

Figure 57: Geocoded Sentinel-1 MLI image of Cornwall, Ontario. 


\section{Appendix C: Ancillary Sentinel-1 Interferograms}

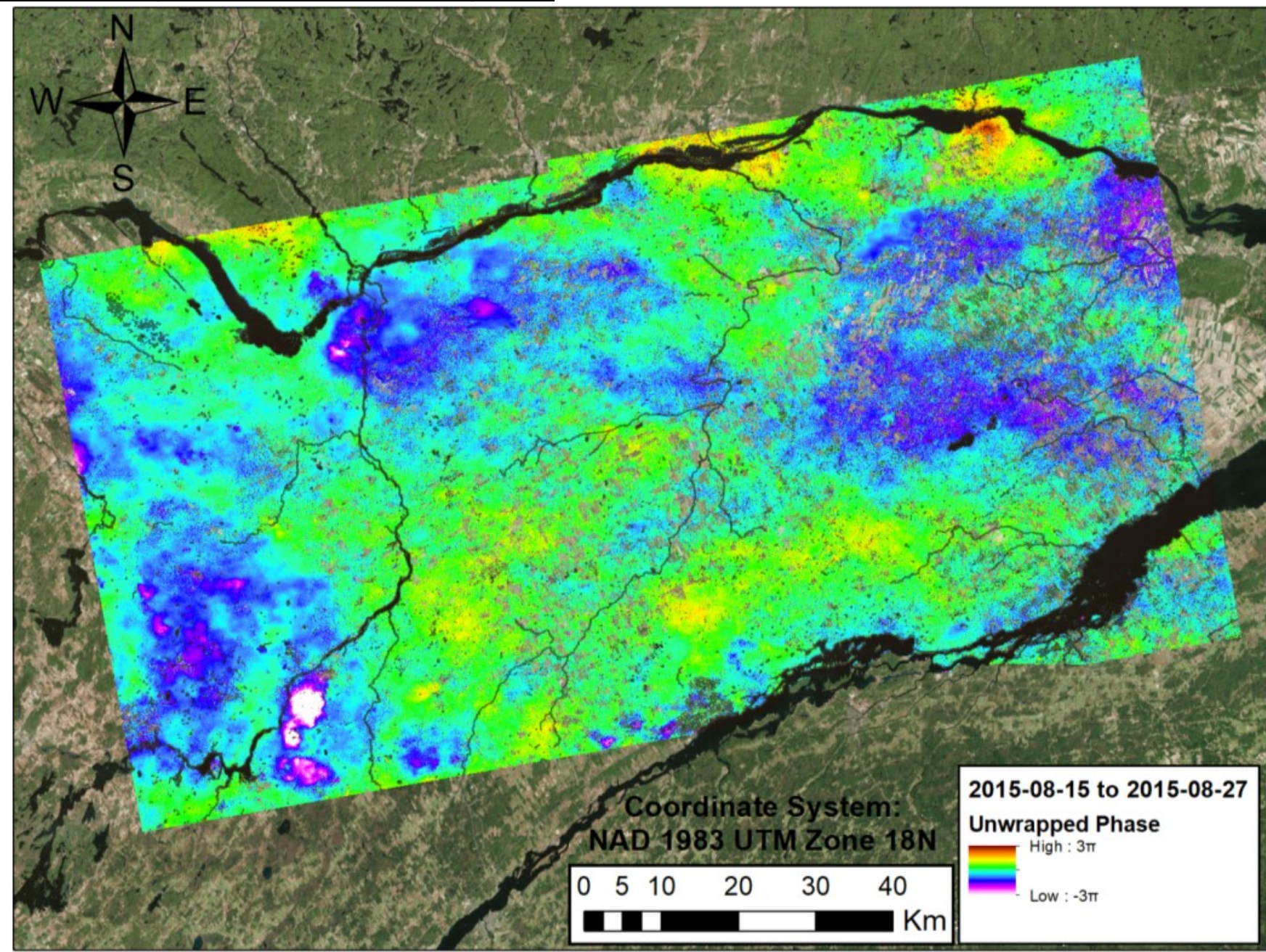

Figure 58: Unwrapped, differential interferogram created from Sentinel-1 summer acquisitions (2018-08-15 and 2015-08-27) with a 12 day temporal baseline. 


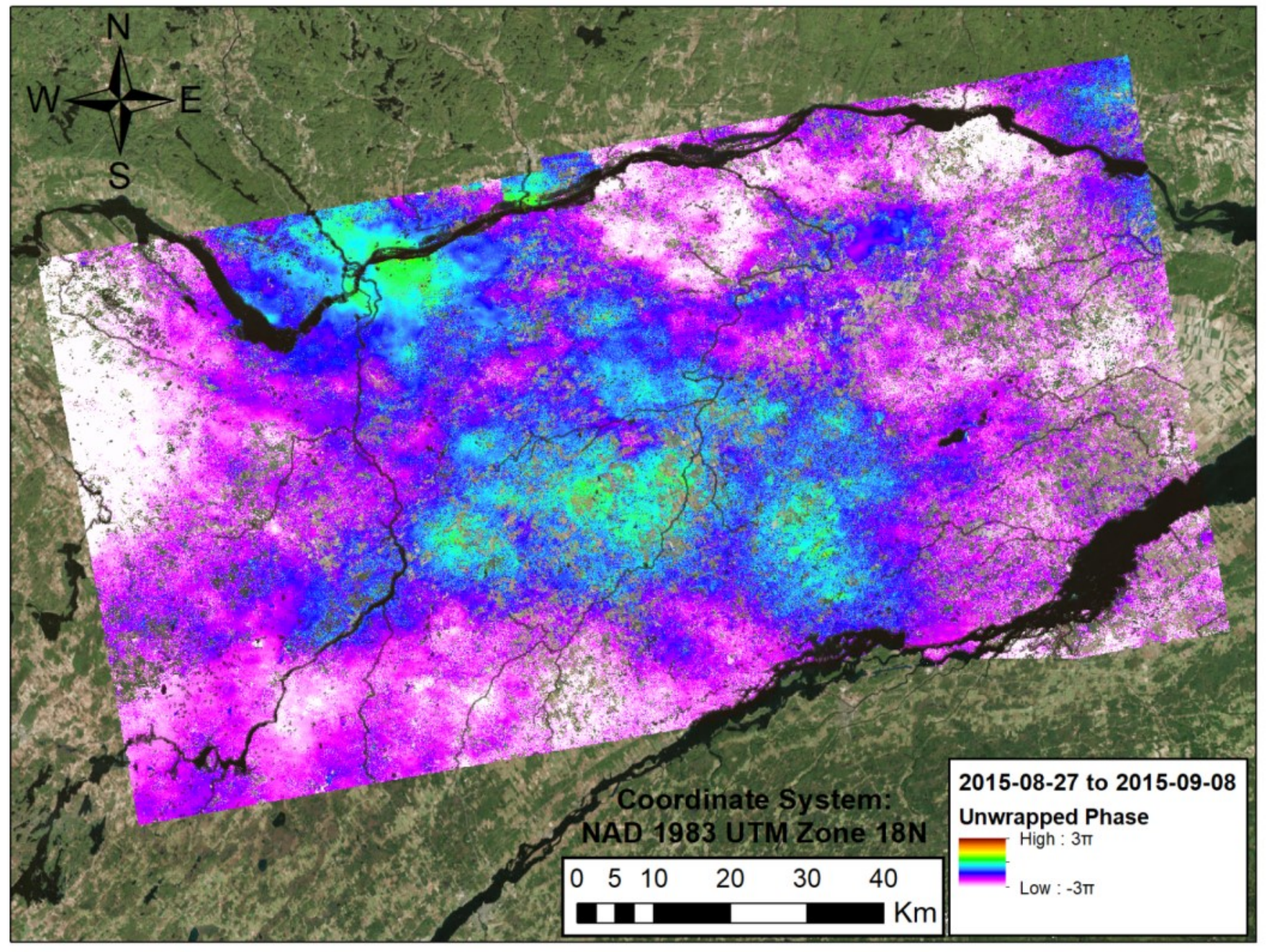

Figure 59: Unwrapped, differential interferogram created from Sentinel-1 summer acquisitions (2015-08-27 and 2015-09-08) with a 12 day temporal baseline. 


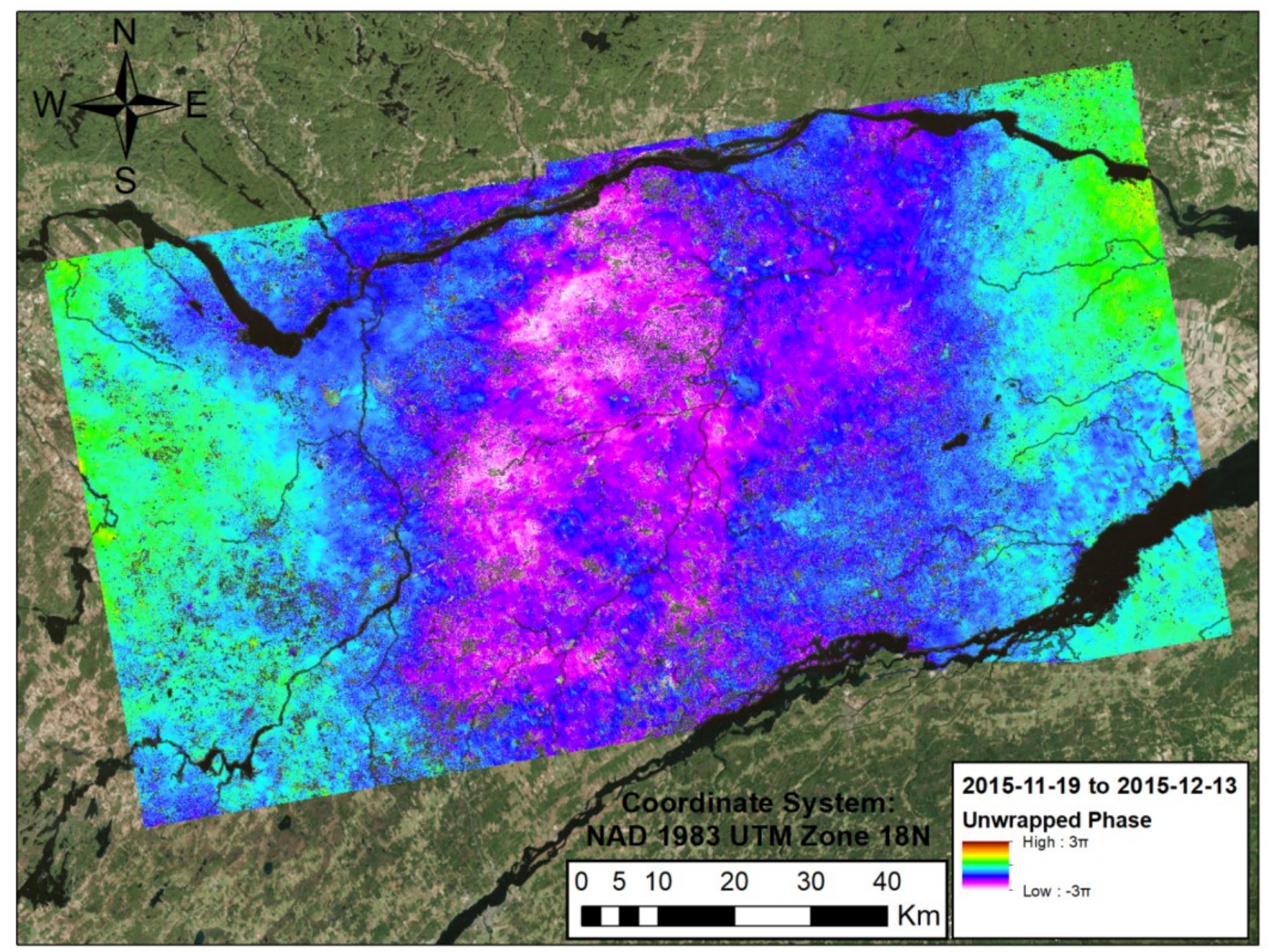

Figure 60: Unwrapped, differential interferogram created from Sentinel-1 winter acquisitions (2015-11-19 and 2015-12-13) with a 24 day temporal baseline. 


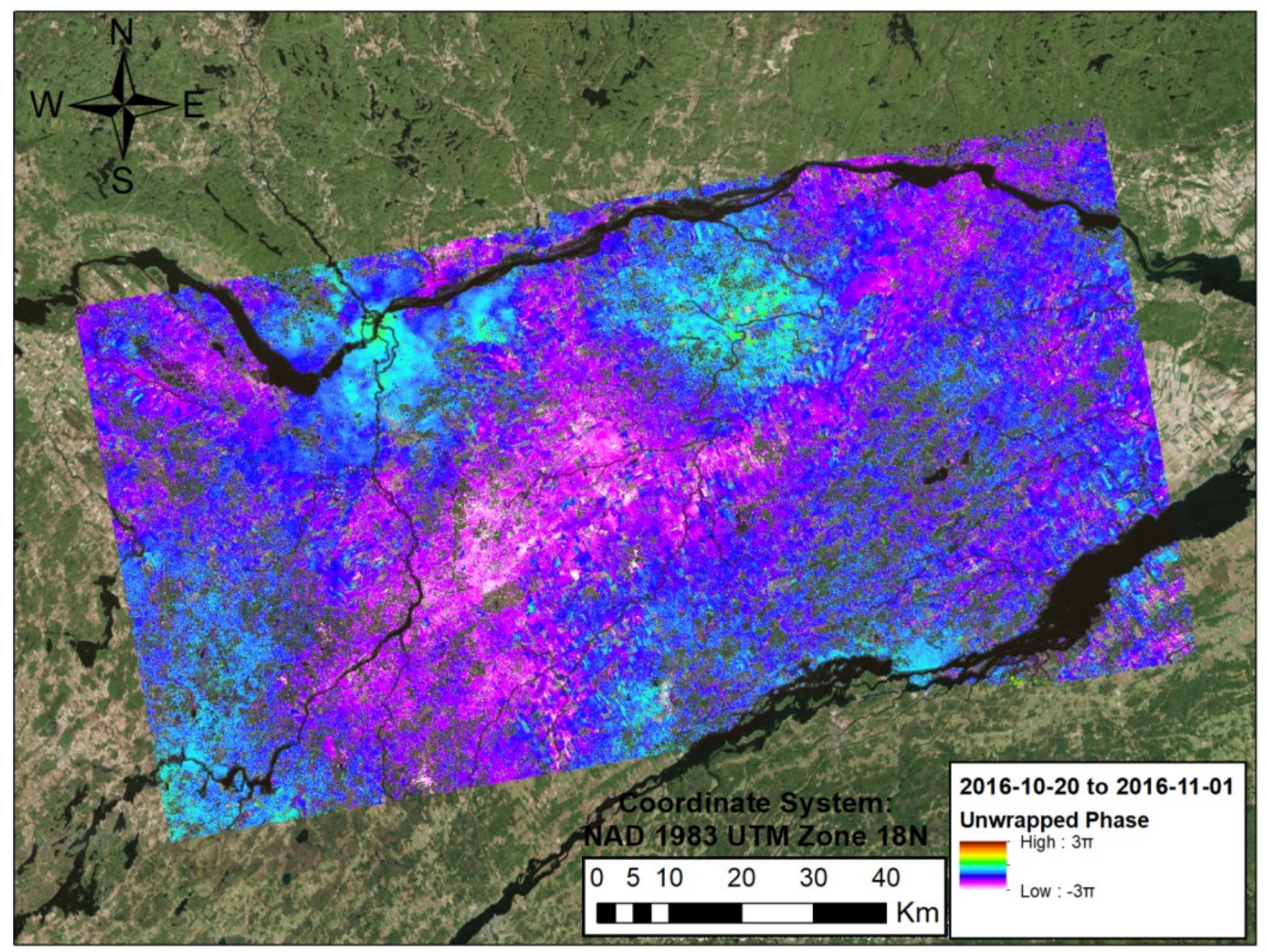

Figure 61: Unwrapped, differential interferogram created from Sentinel-1 fall acquisitions (2016-10-20 and 2016-11-01) with a 12 day temporal baseline. 


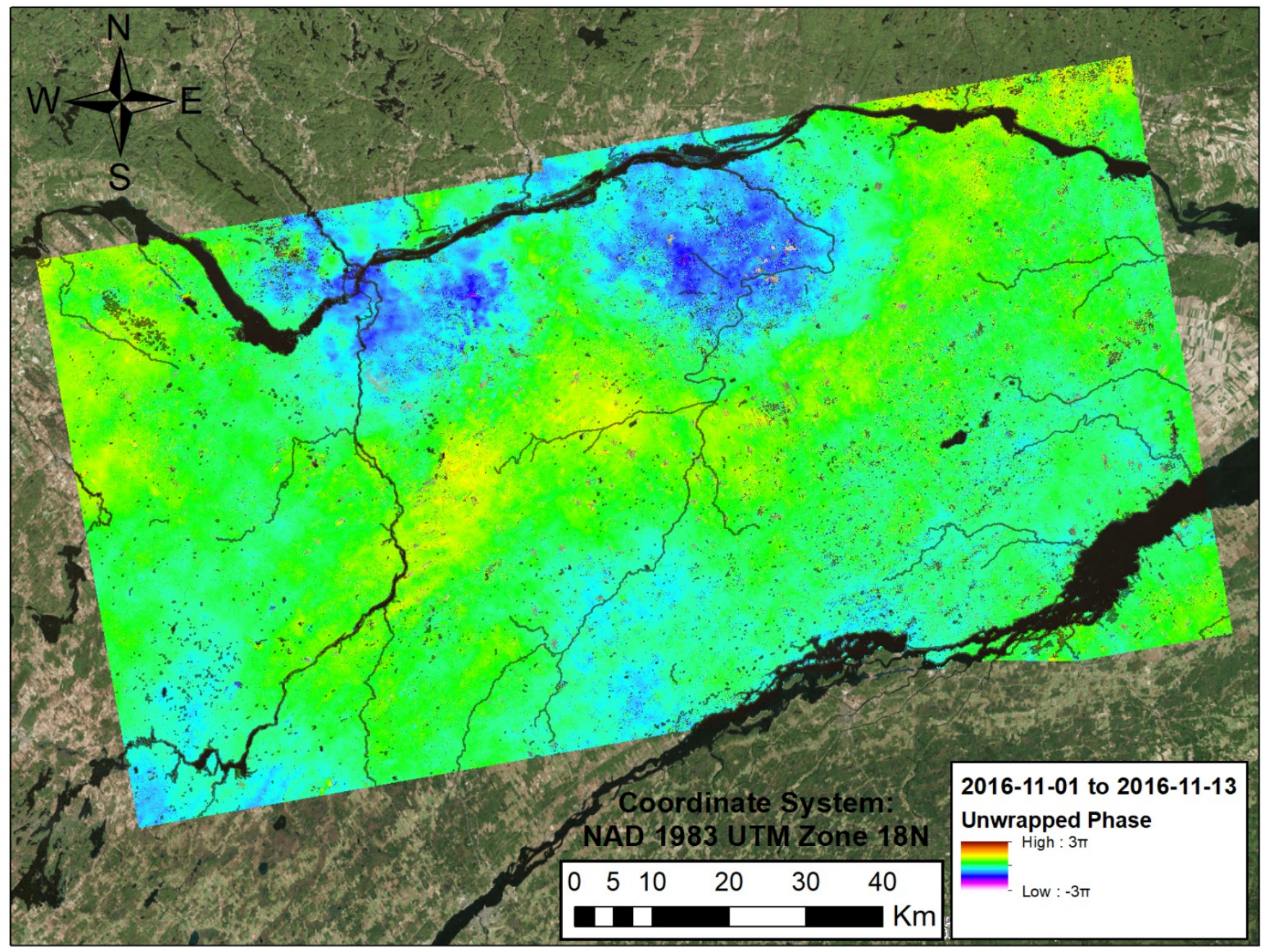

Figure 62: Unwrapped, differential interferogram created from Sentinel-1 fall acquisitions (2016-11-01 and 2016-11-13) with a 12 day temporal baseline. 


\section{Appendix D: Sentinel-1 Coherence Maps}

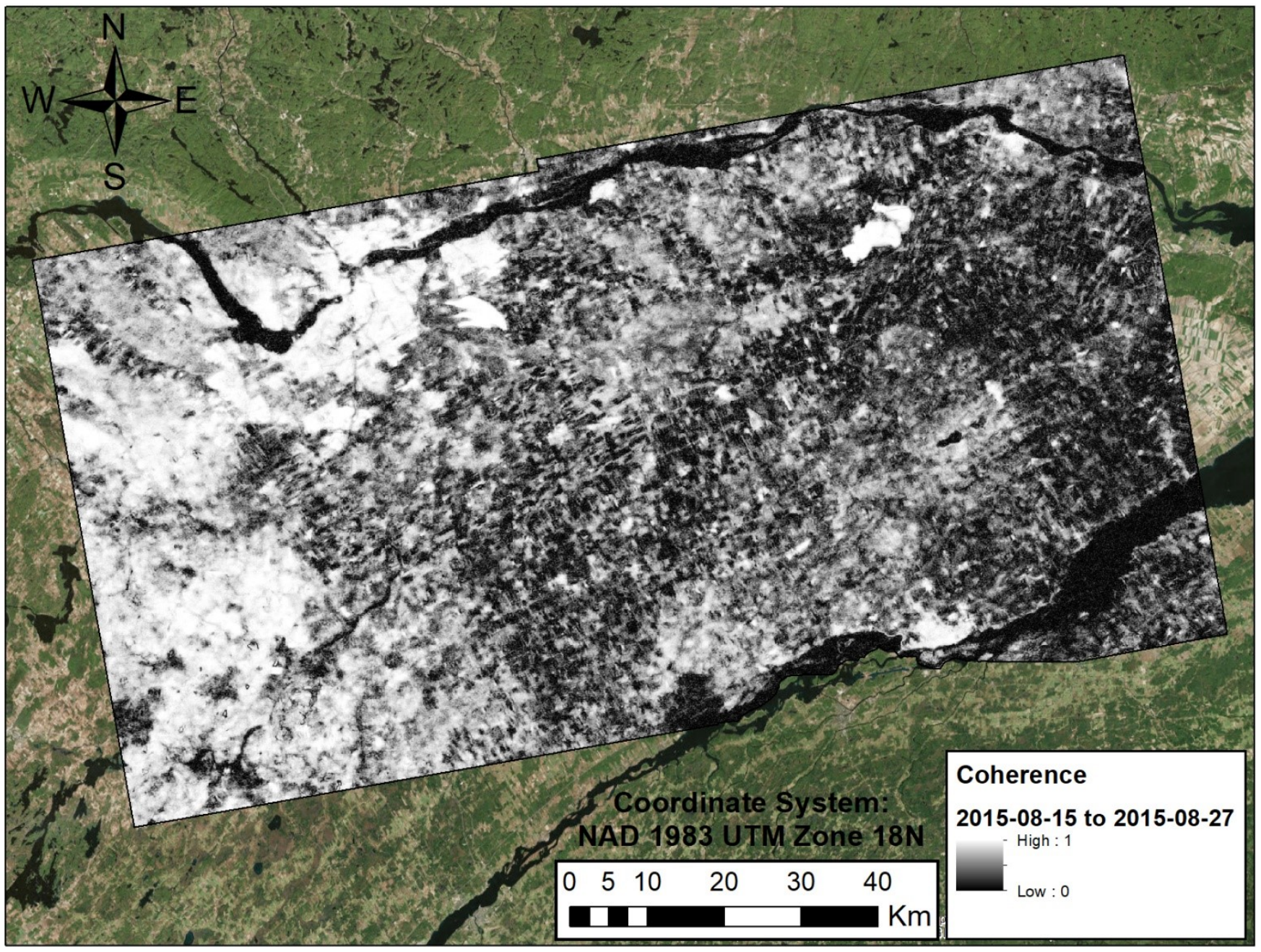

Figure 63: Interferometric coherence of the 2015-08-15 to 2015-08-27 Sentinel-1 interferogram. 


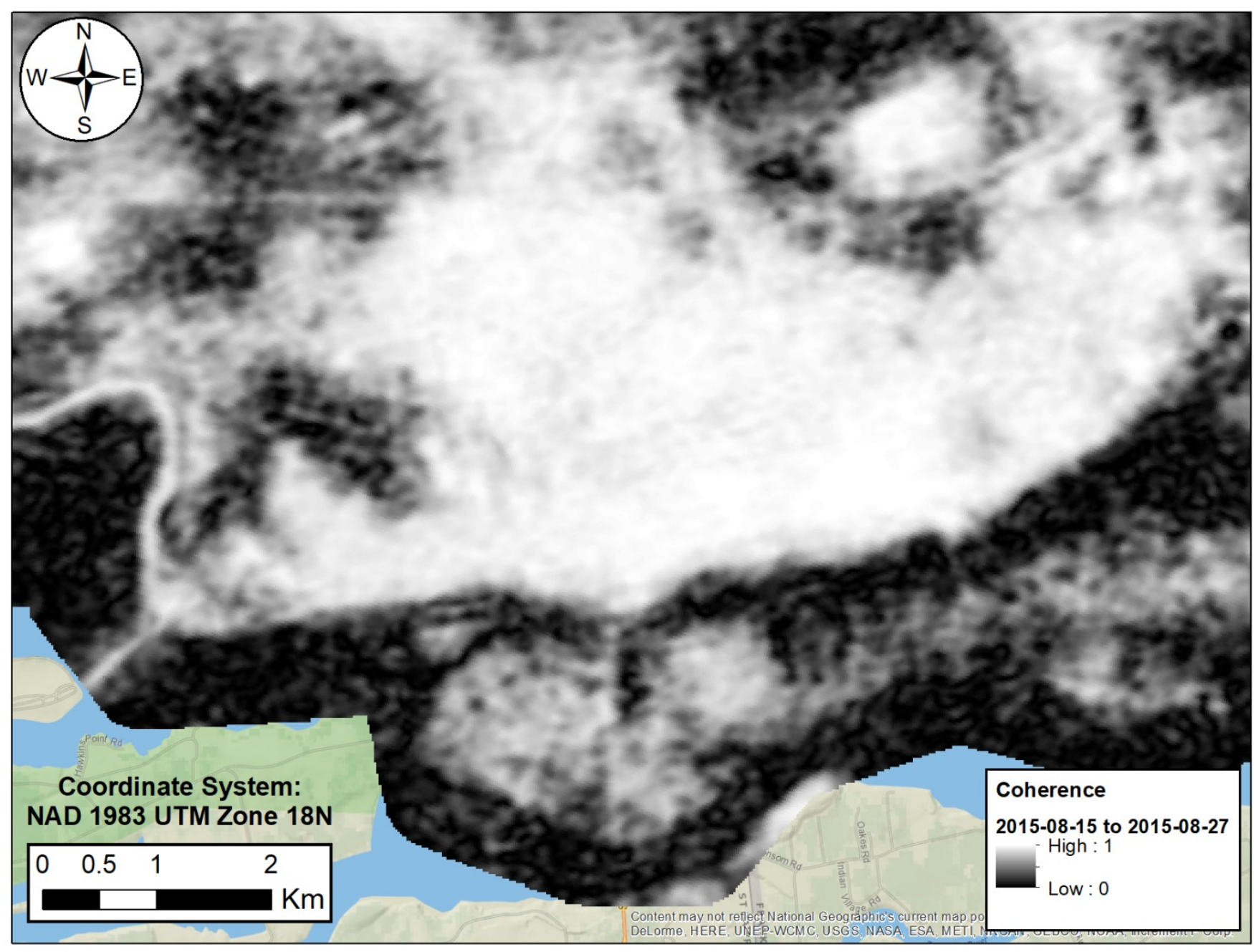

Figure 64: Interferometric coherence of Cornwall of the 2015-08-15 to 2015-08-27 Sentinel-1 interferogram. 


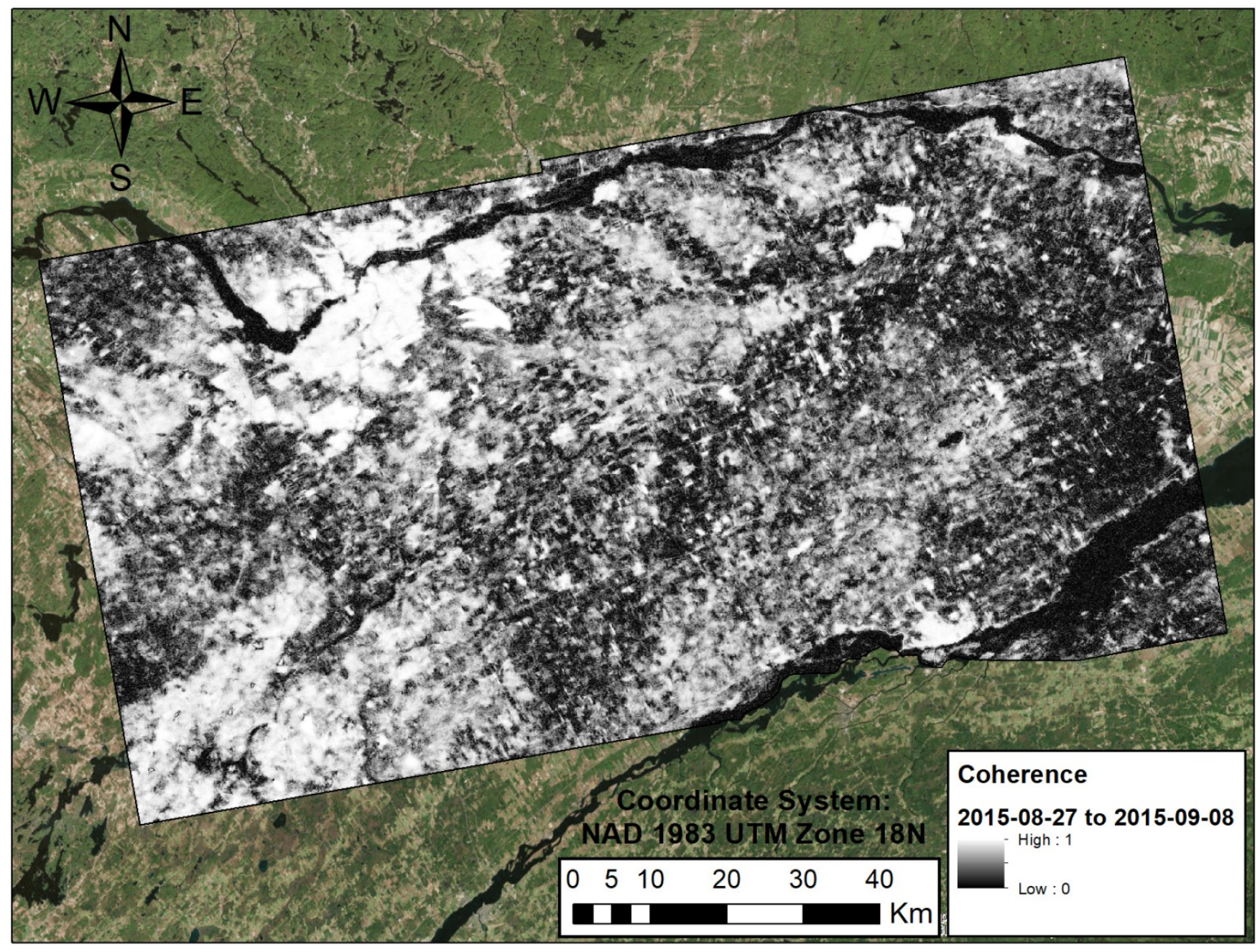

Figure 65: Interferometric coherence of the 2015-08-27 to 2015-09-08 Sentinel-1 interferogram. 


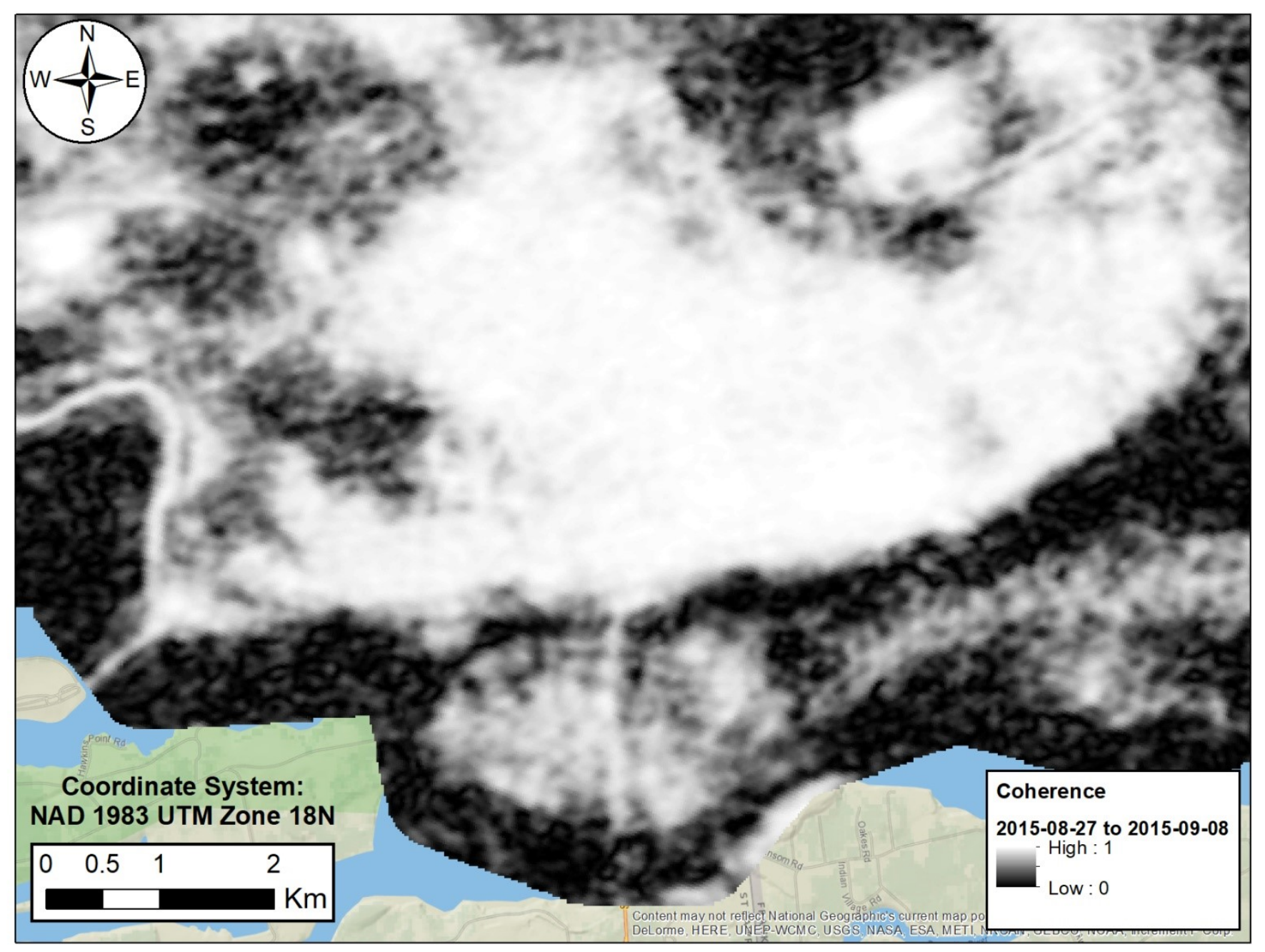

Figure 66: Interferometric coherence of Cornwall of the 2015-08-27 to 2015-09-08 Sentinel-1 interferogram. 


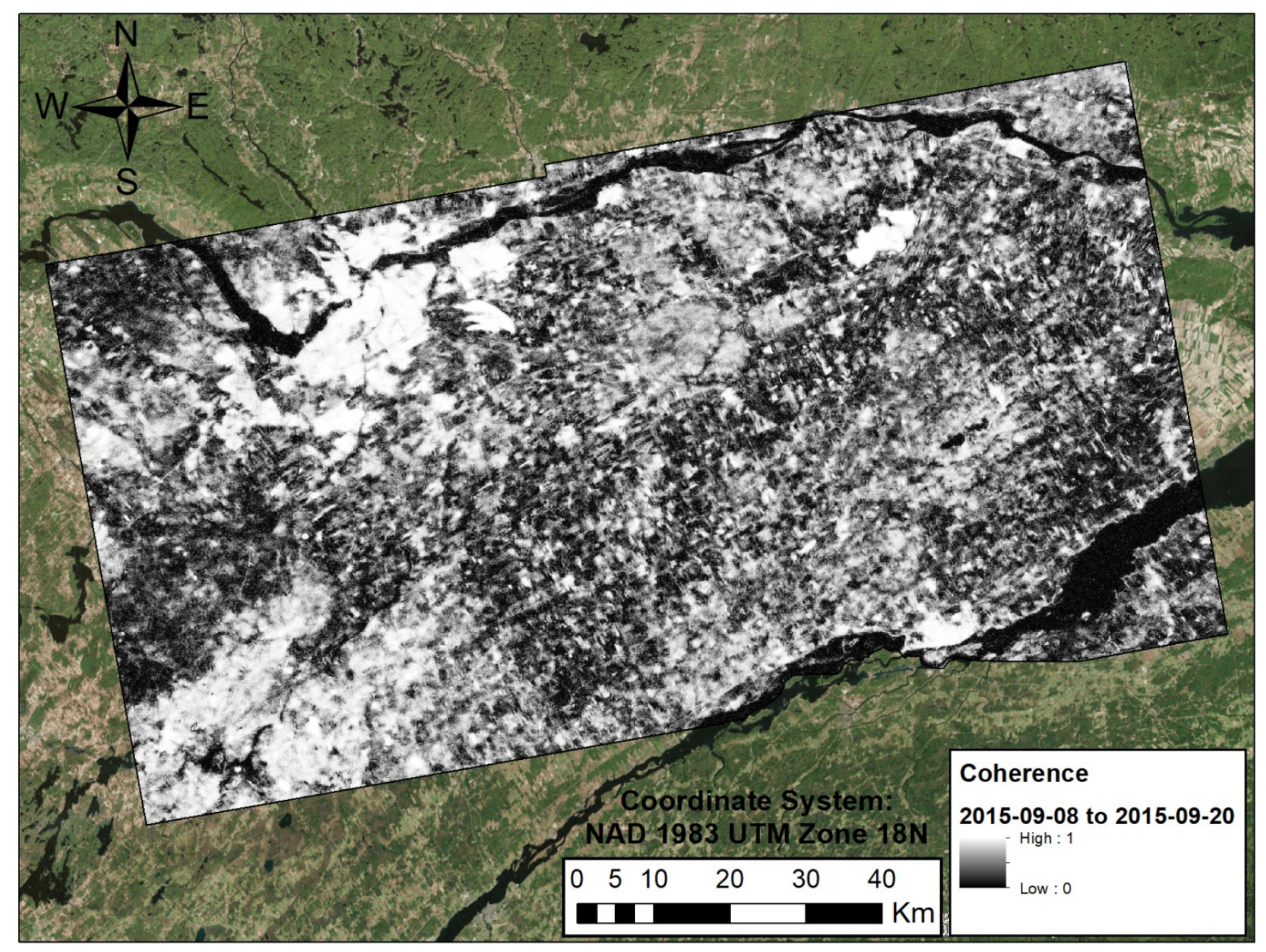

Figure 67: Interferometric coherence of the 2015-09-08 to 2015-09-20 Sentinel-1interferogram. 


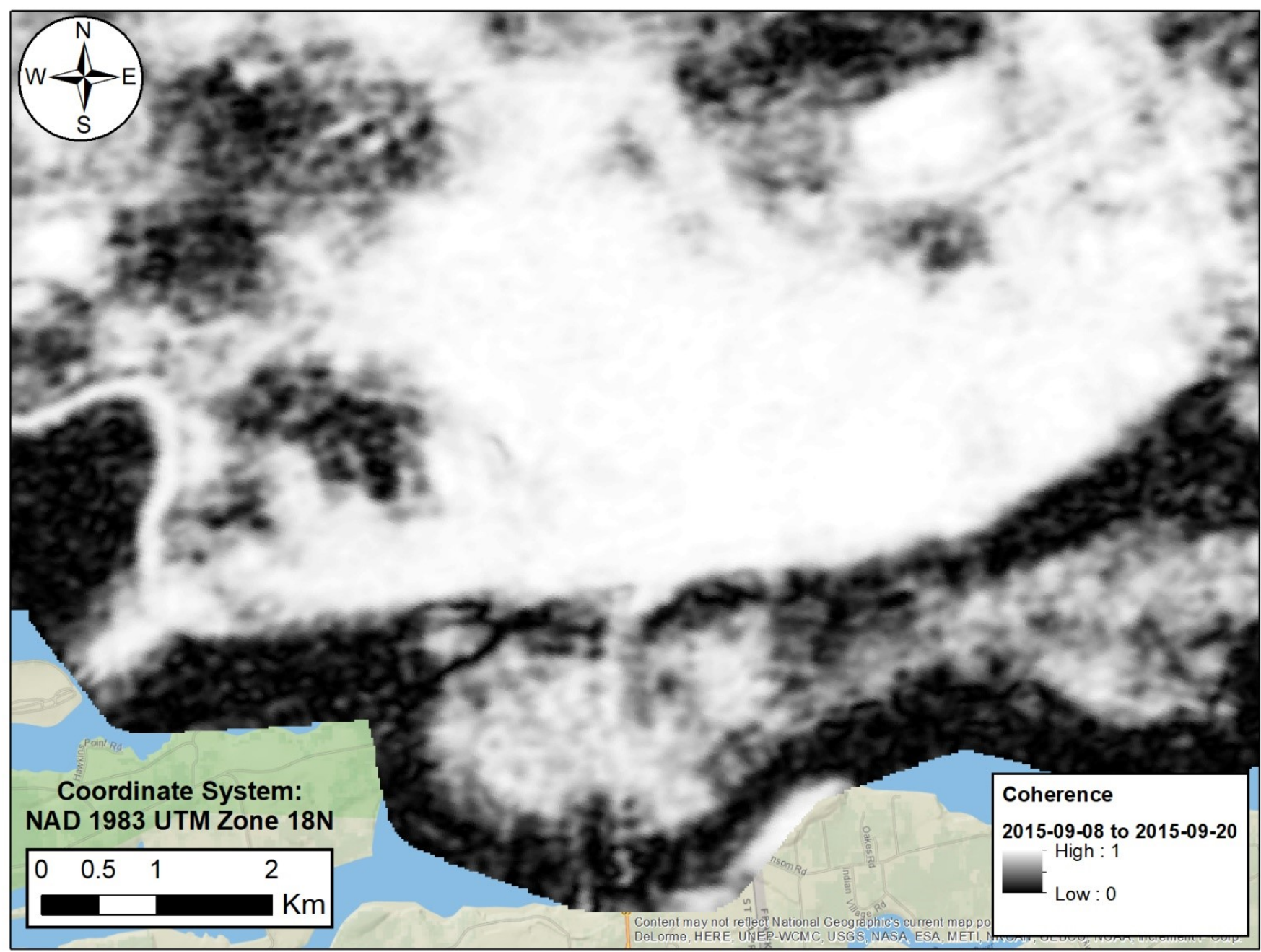

Figure 68: Interferometric coherence of Cornwall of the 2015-09-08 to 2015-09-20 Sentinel-1interferogram. 


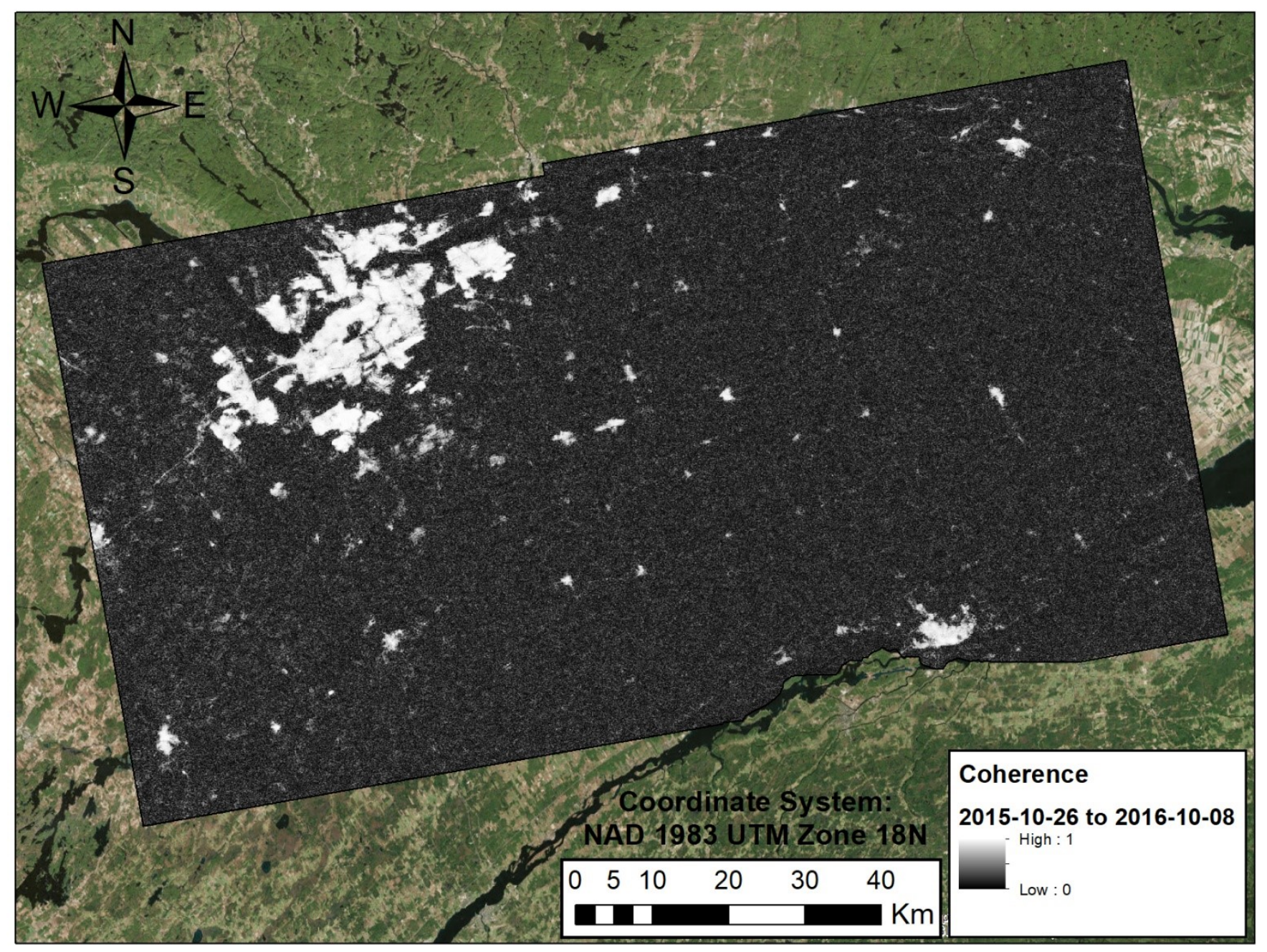

Figure 69: Interferometric coherence of the 2015-10-26 to 2016-10-08 Sentinel-1interferogram. 


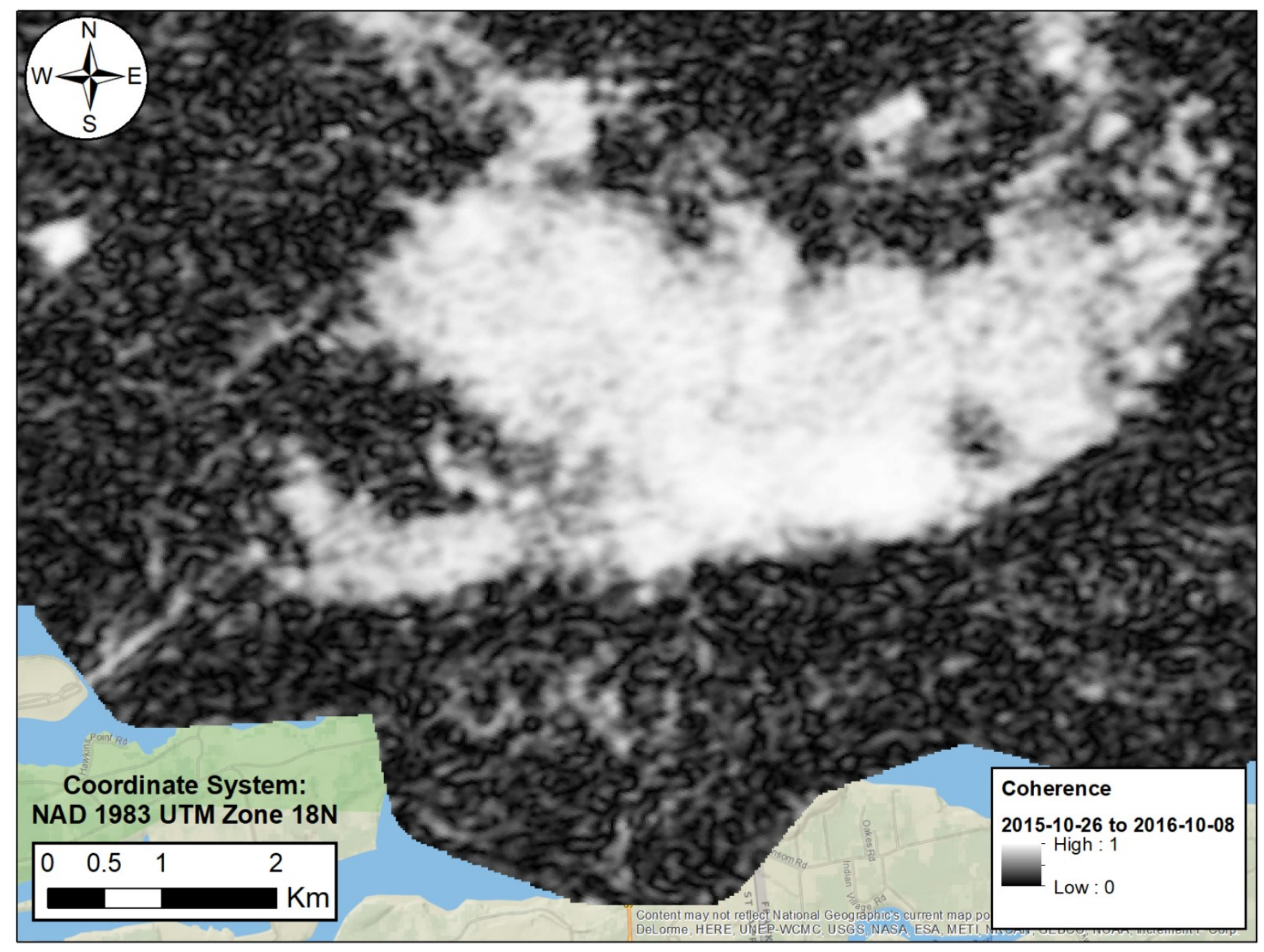

Figure 70: Interferometric coherence of Cornwall of the 2015-10-26 to 2016-10-08 Sentinel-1 interferogram. 


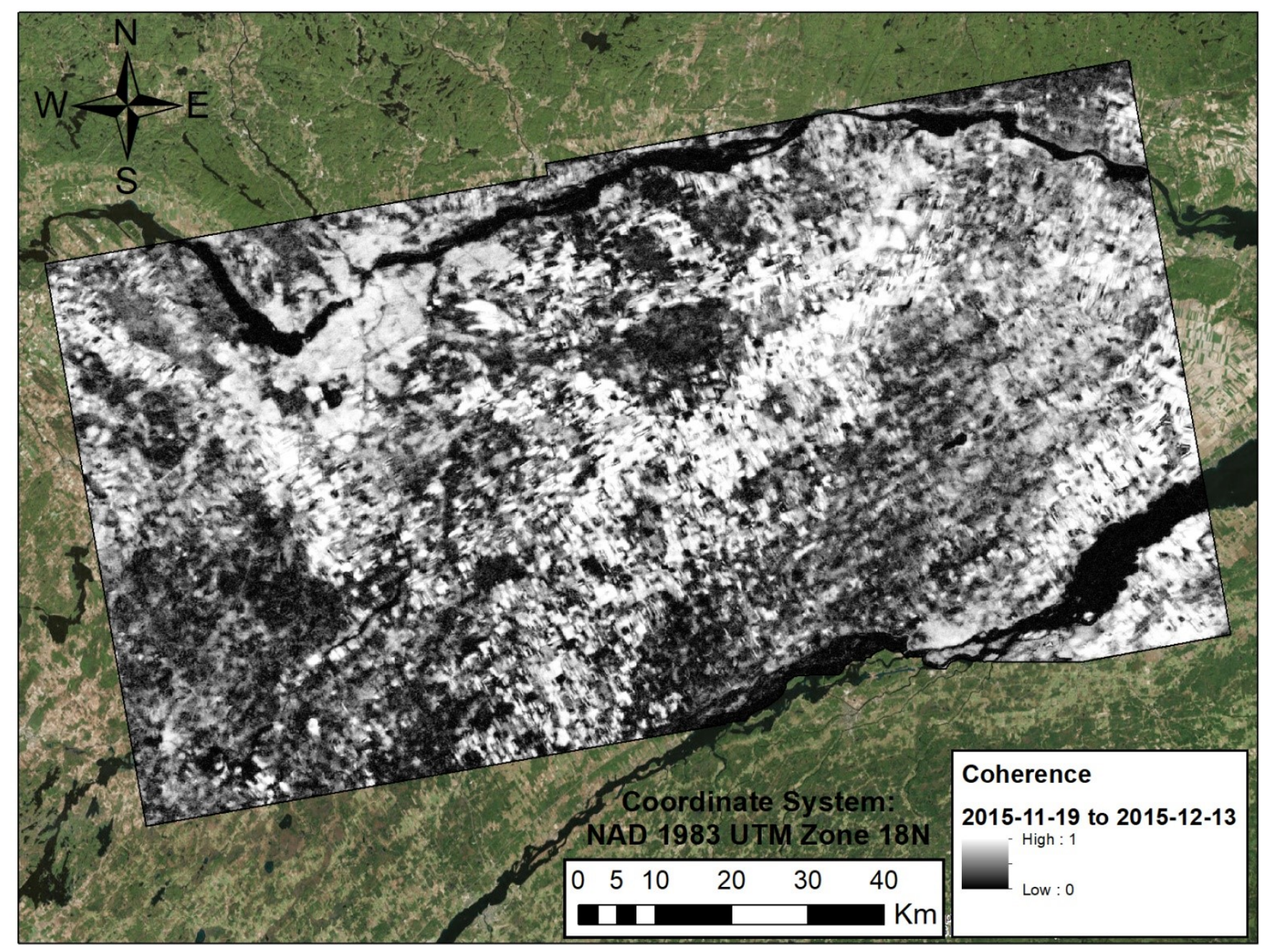

Figure 71: Interferometric coherence of the 2015-11-19 to 2015-12-13 Sentinel-1interferogram. 


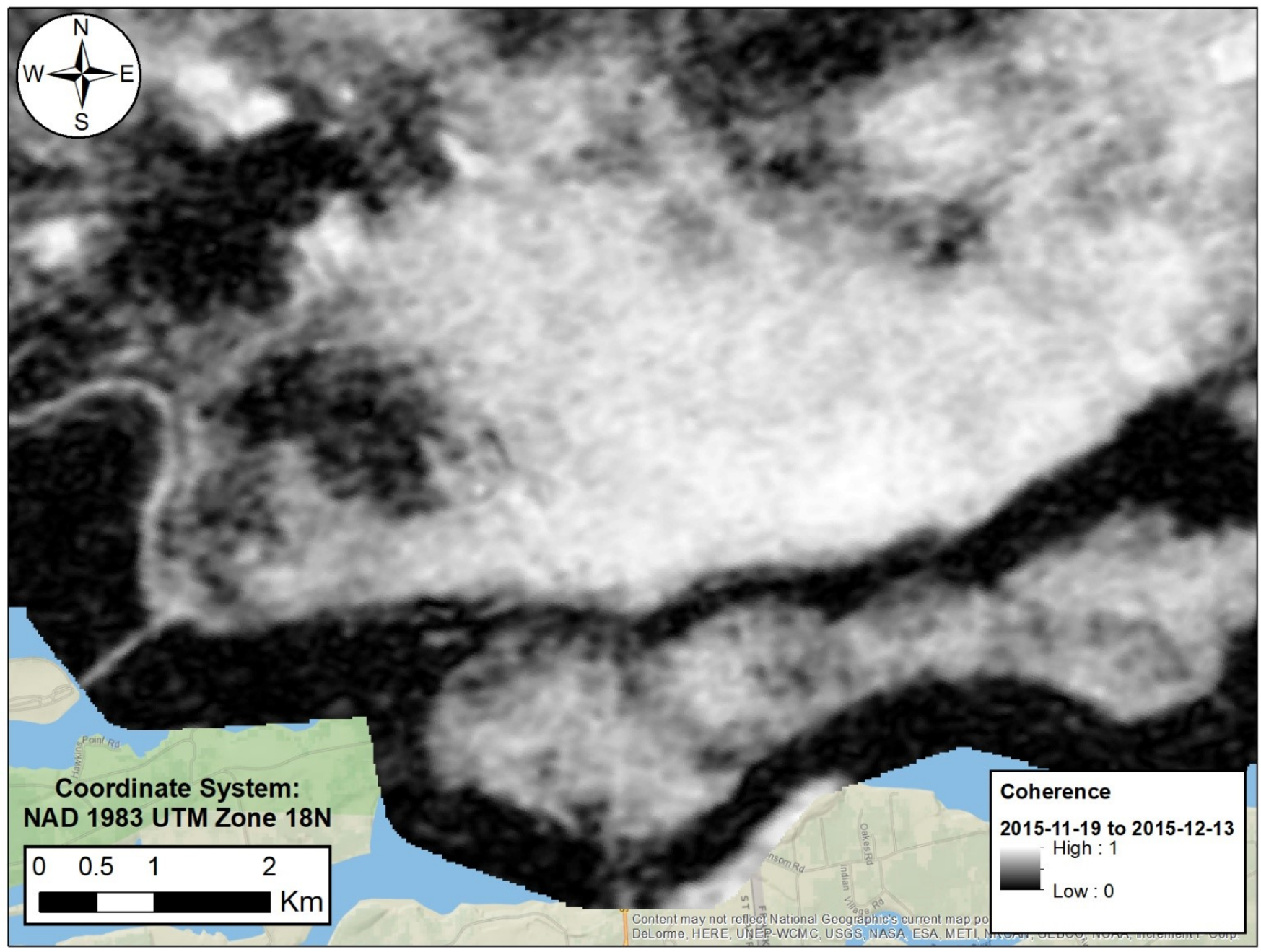

Figure 72: Interferometric coherence of Cornwall of the 2015-11-19 to 2015-12-13 Sentinel-1interferogram. 


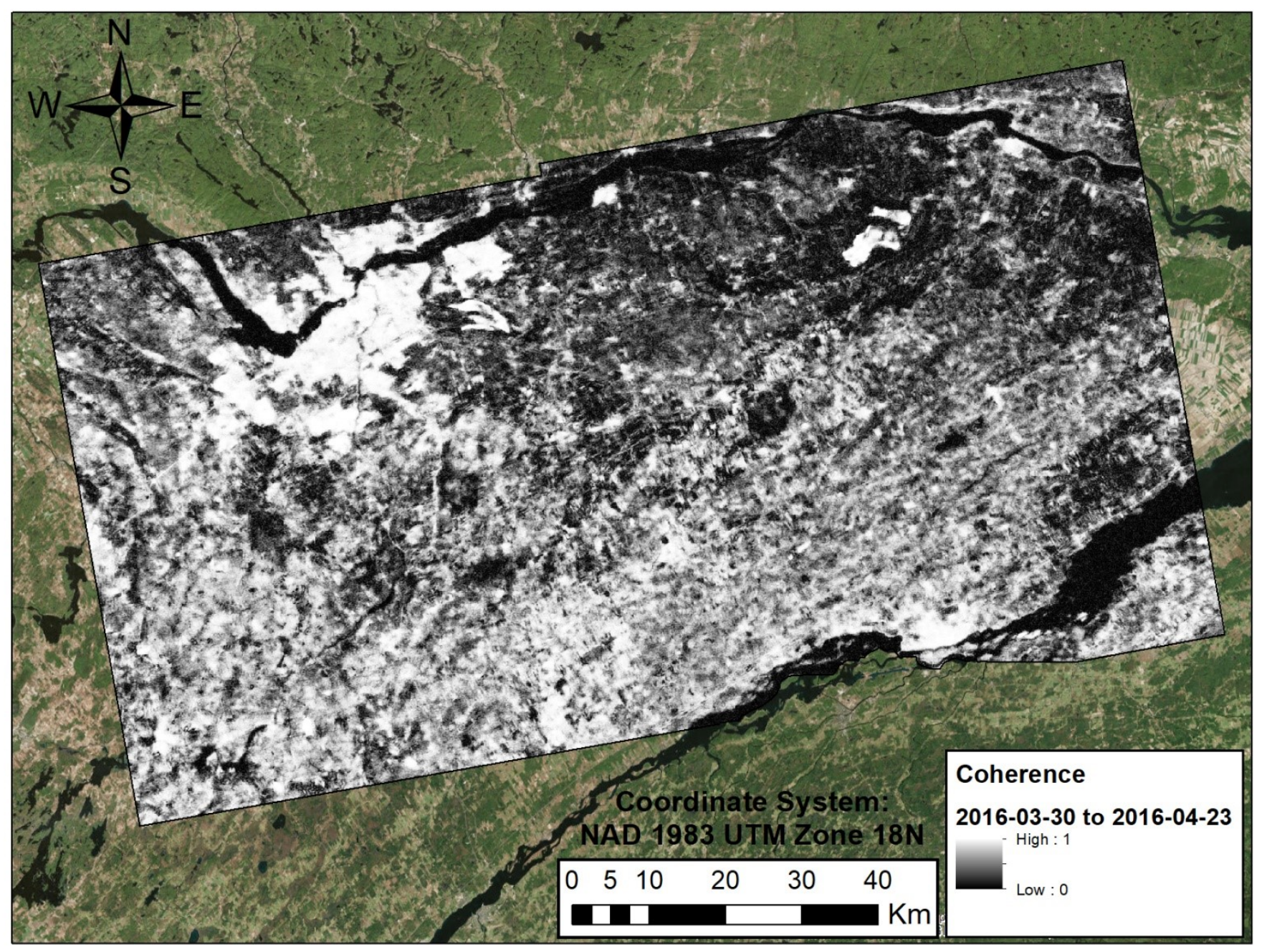

Figure 73: Interferometric coherence of the 2016-03-30 to 2016-04-23 Sentinel-1 interferogram. 


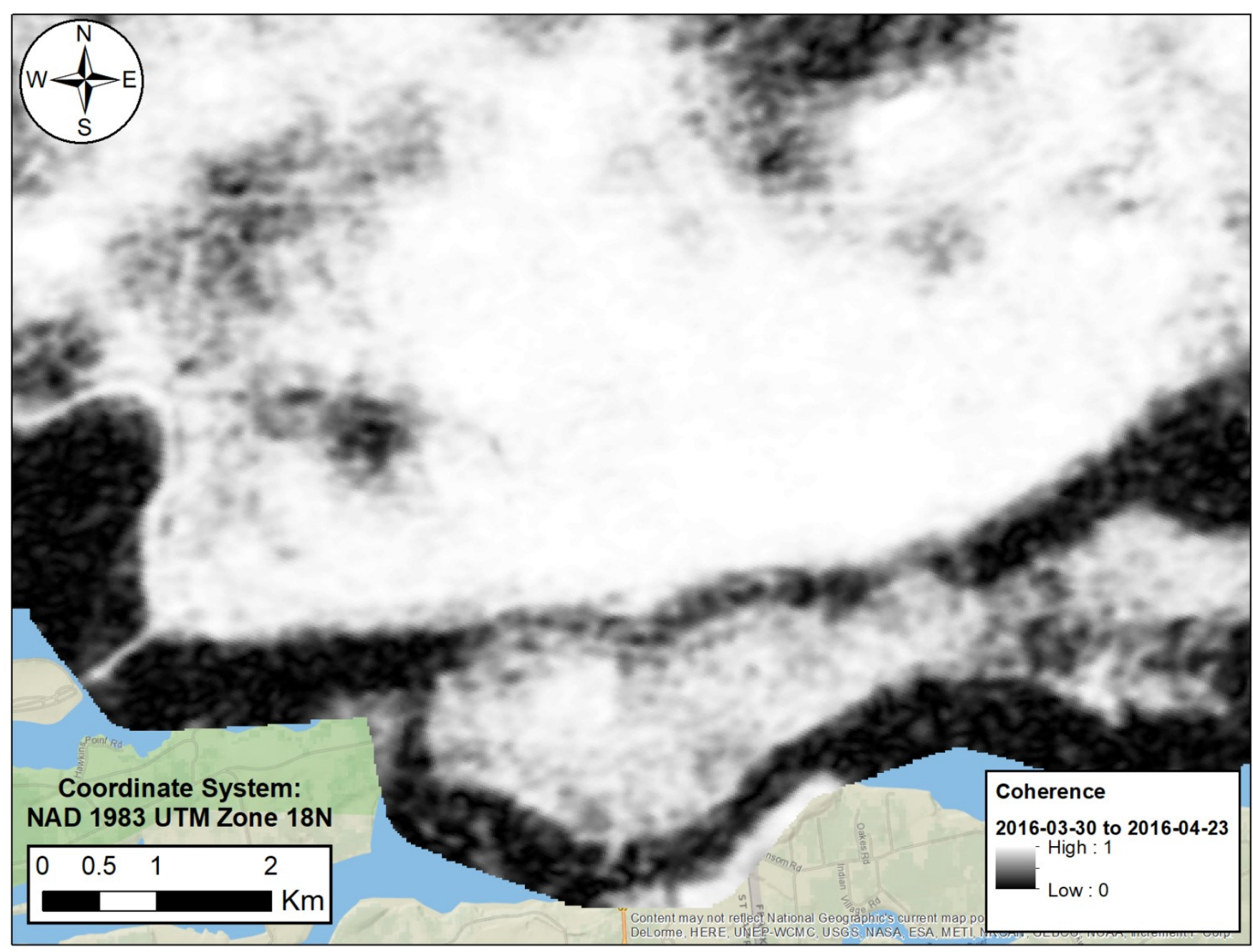

Figure 74: Interferometric coherence of Cornwall of the 2016-03-30 to 2016-04-23 Sentinel-1 interferogram. 


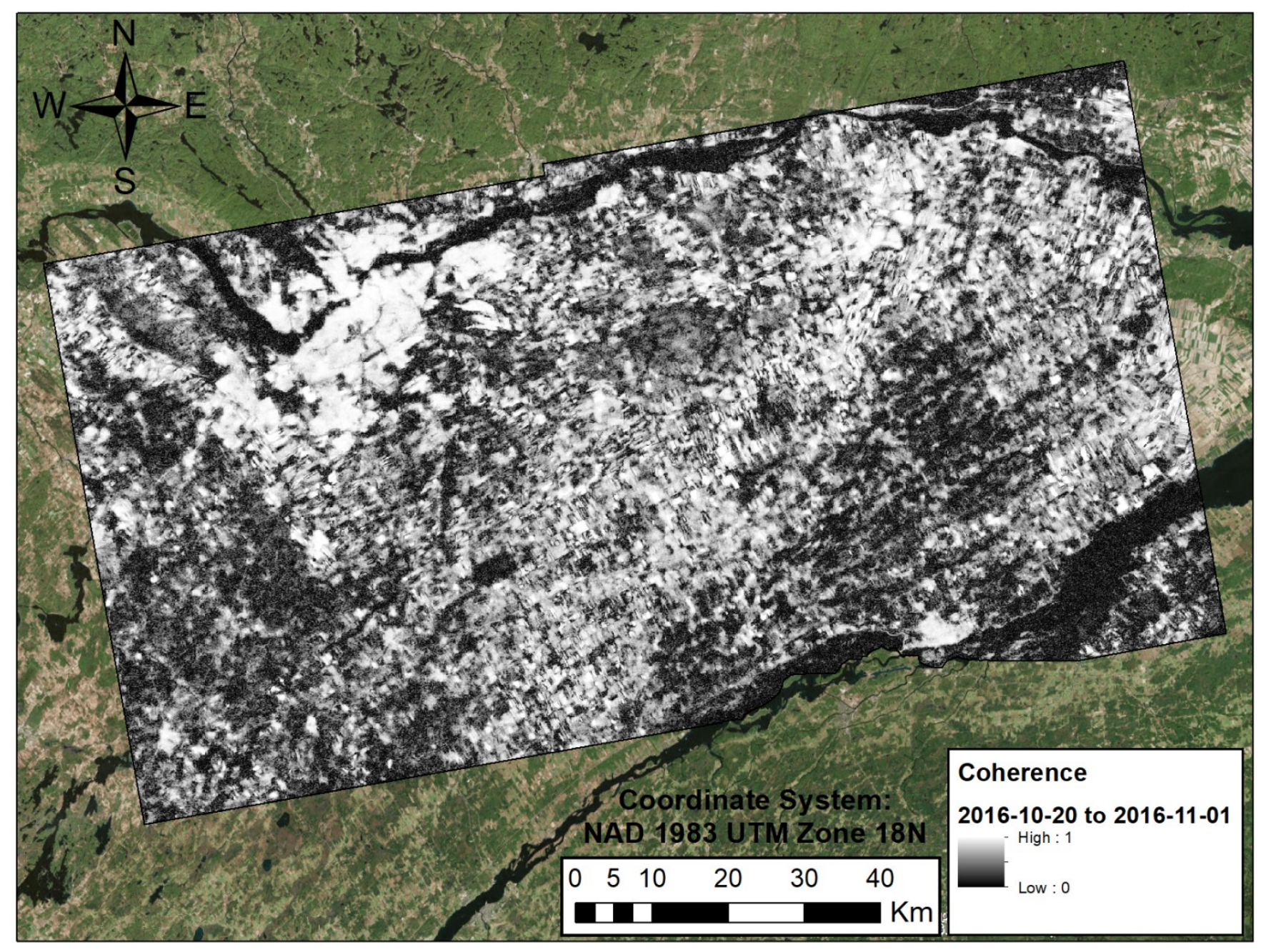

Figure 75: Interferometric coherence of the 2016-10-20 to 2016-11-01 Sentinel-1 interferogram. 


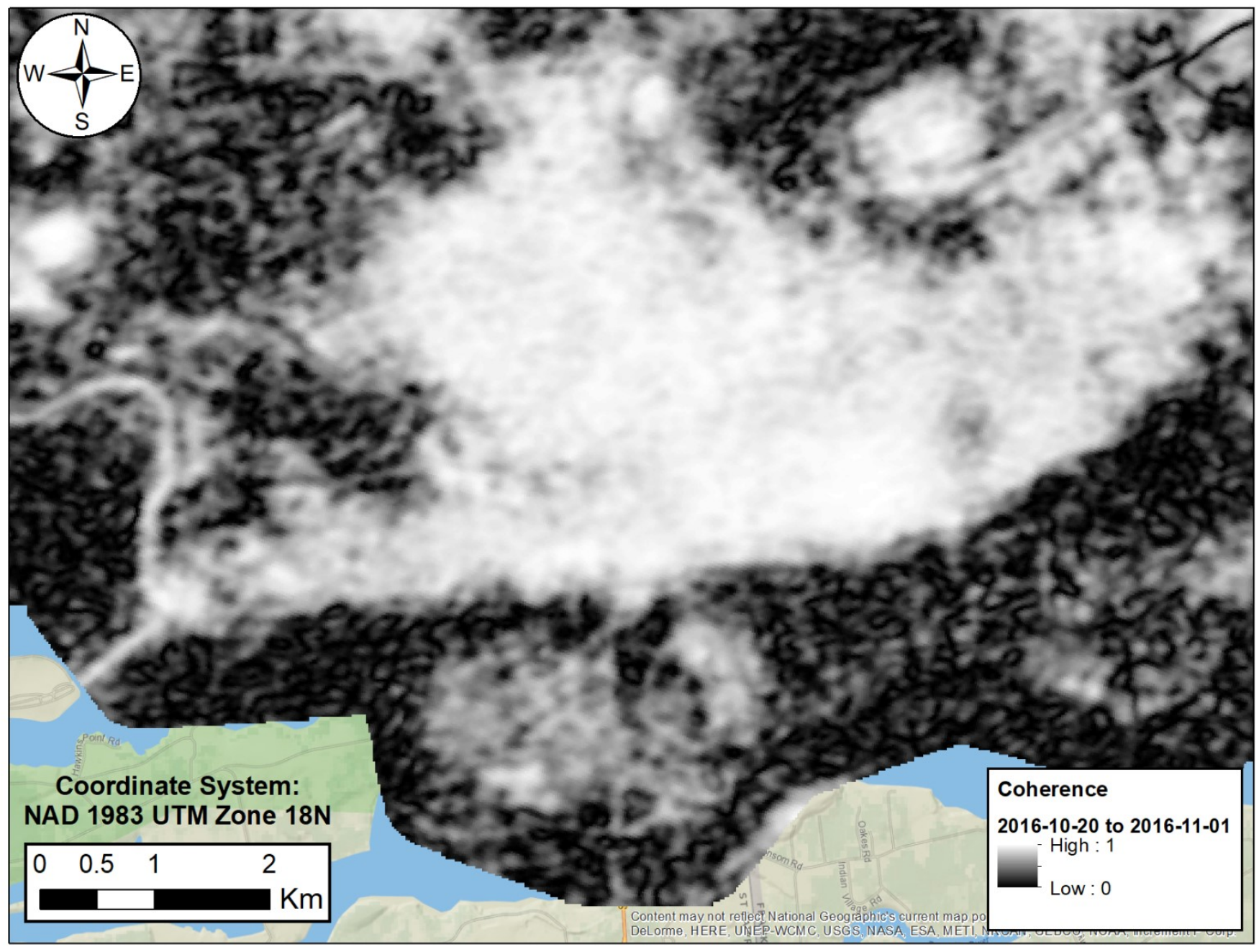

Figure 76: Interferometric coherence of Cornwall of the 2016-10-20 to 2016-11-01 Sentinel-1 interferogram. 


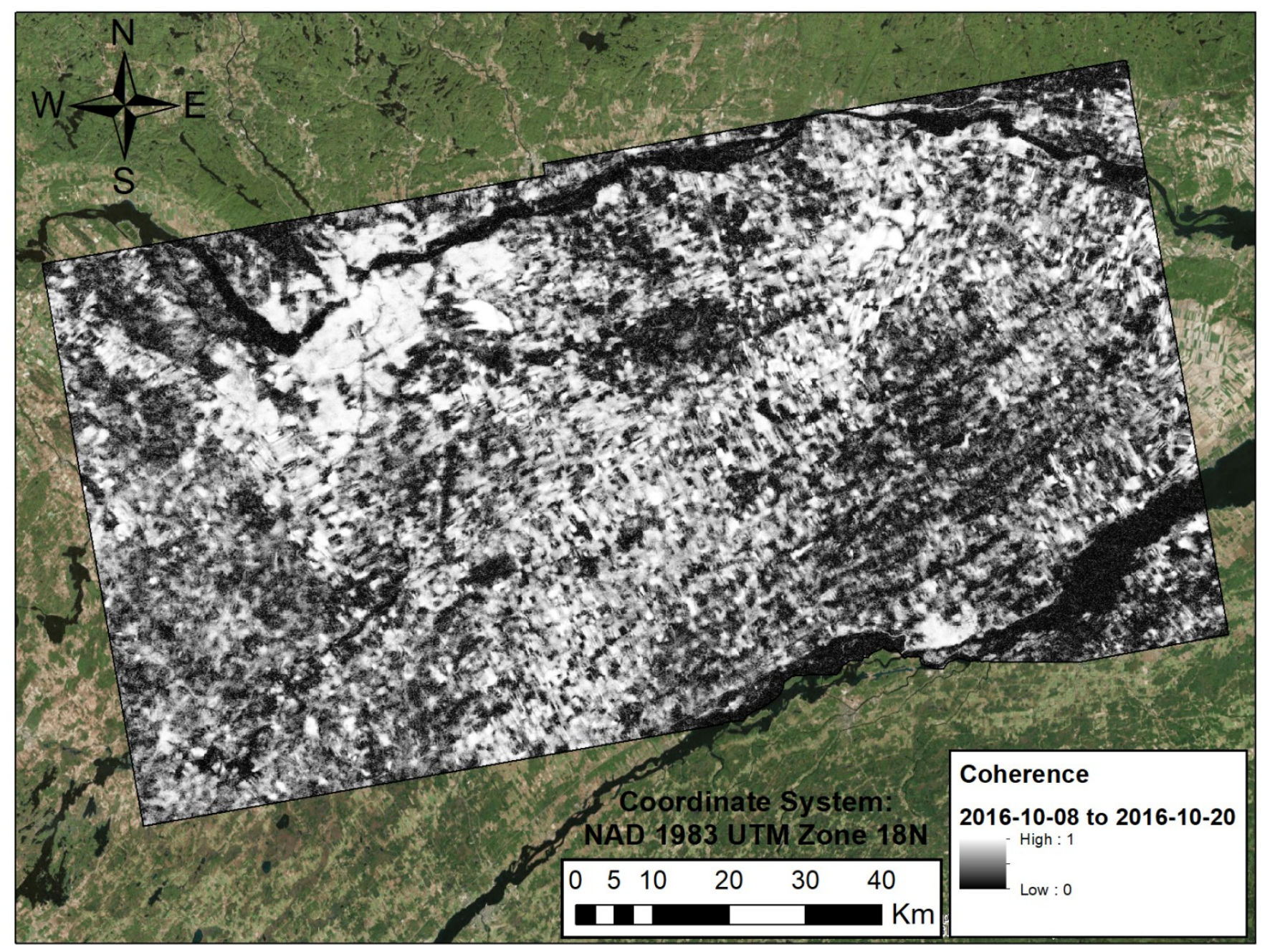

Figure 77: Interferometric coherence of the 2016-10-08 to 2016-10-20 Sentinel-1 interferogram. 


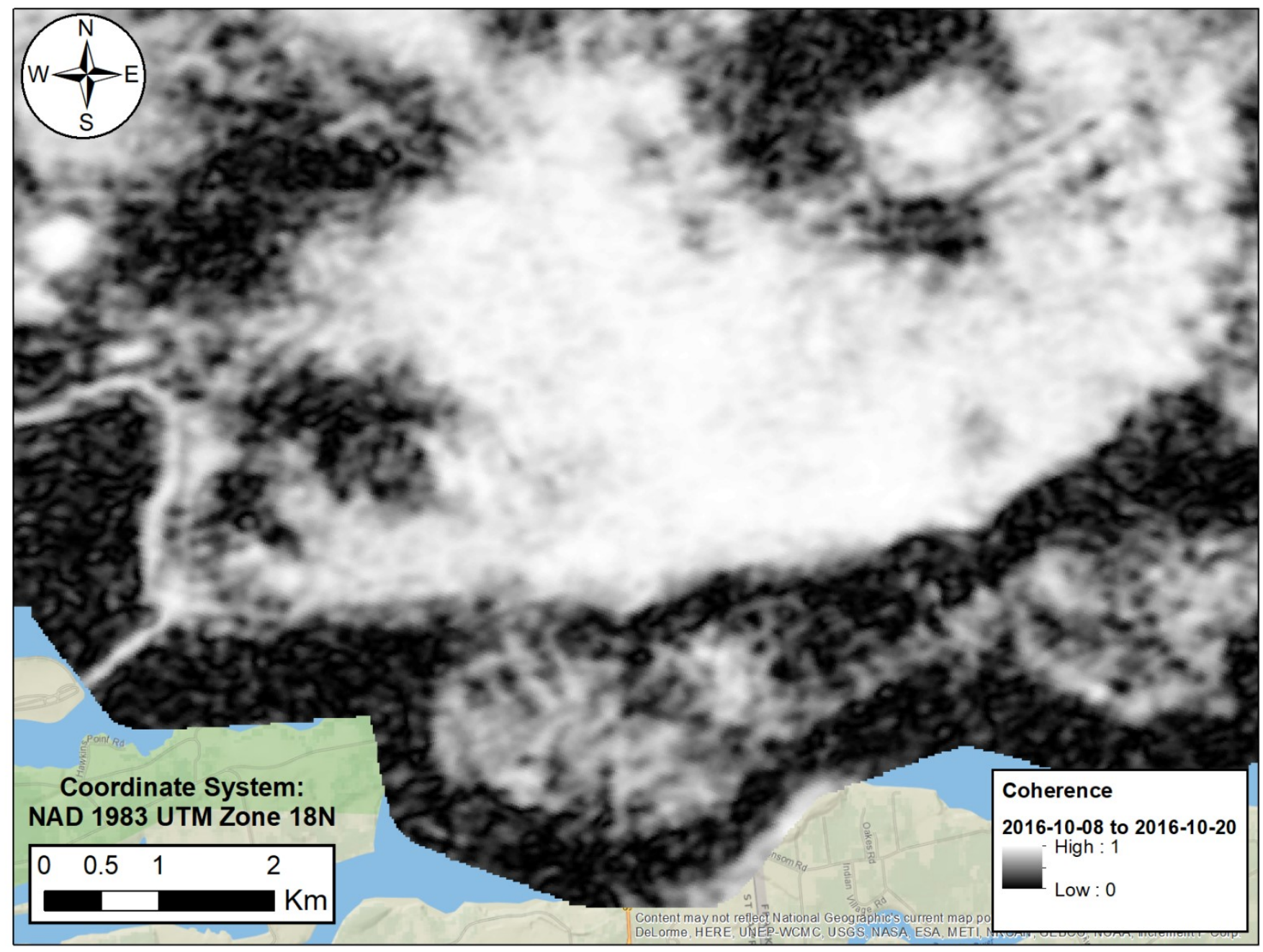

Figure 78: Interferometric coherence of Cornwall of the 2016-10-08 to 2016-10-20 Sentinel-1 interferogram. 


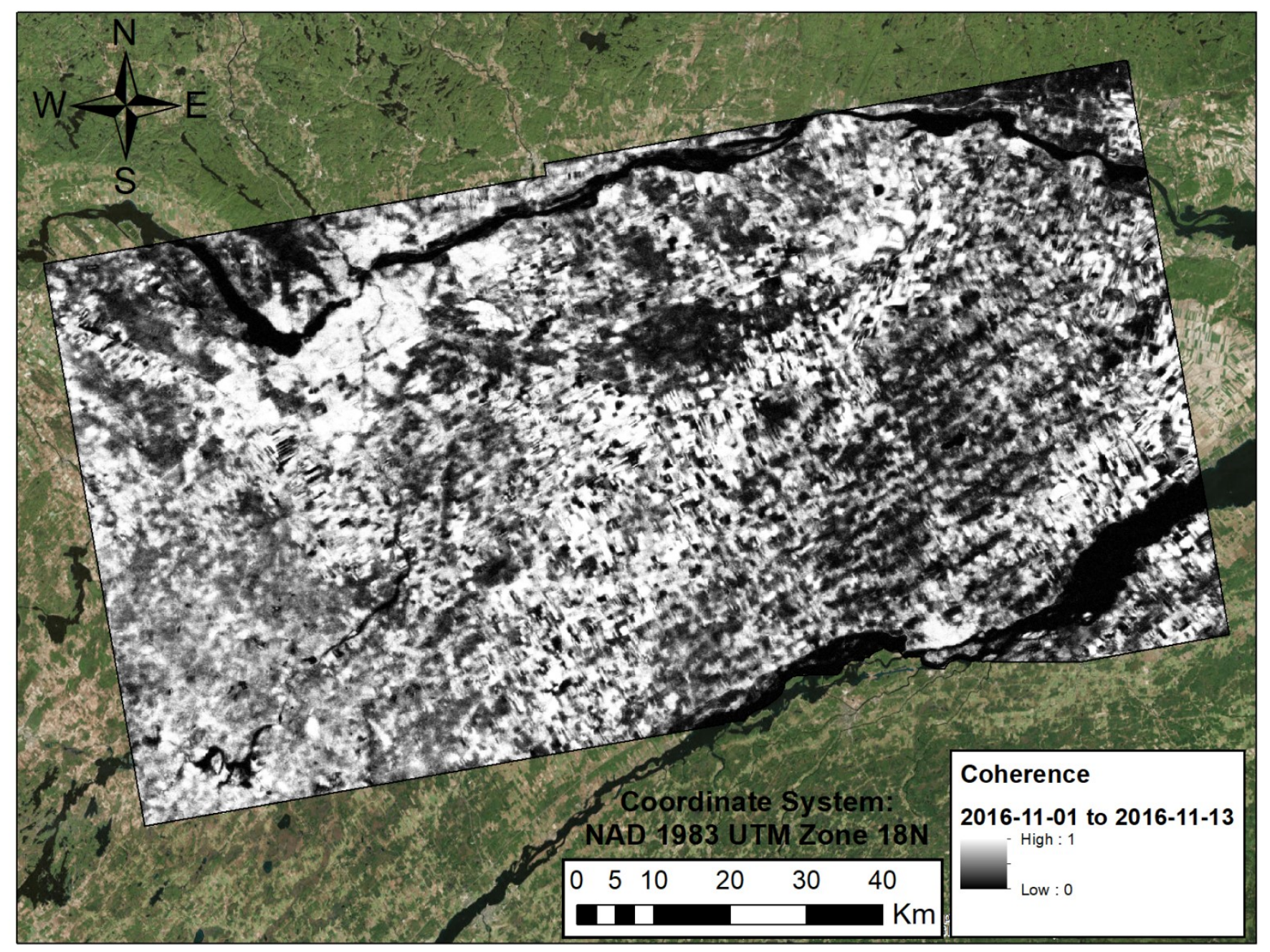

Figure 79: Interferometric coherence of the 2016-11-01 to 2016-11-13 Sentinel-1 interferogram. 


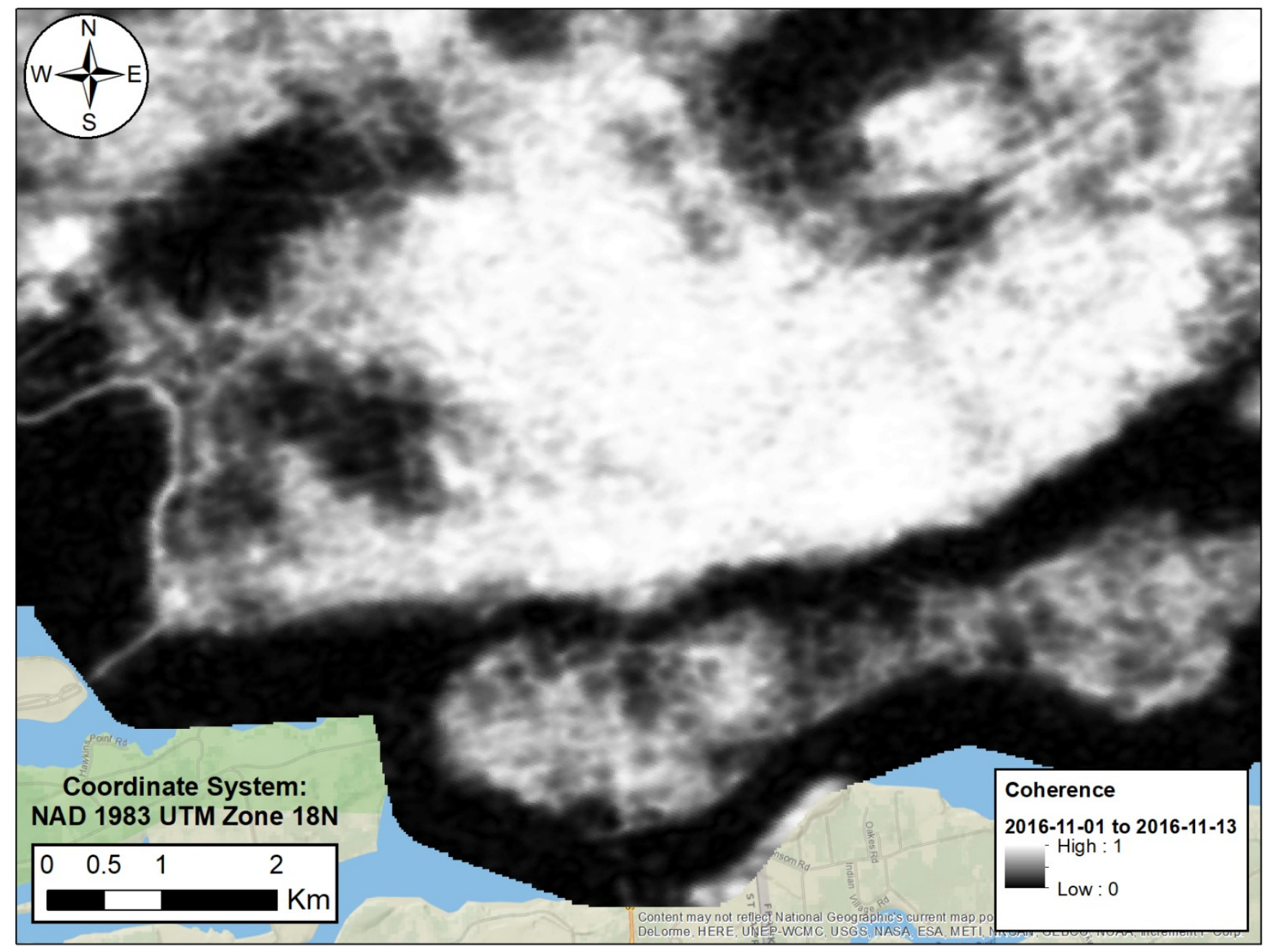

Figure 80: Interferometric coherence of Cornwall of the 2016-11-01 to 2016-11-13 Sentinel-1 interferogram. 


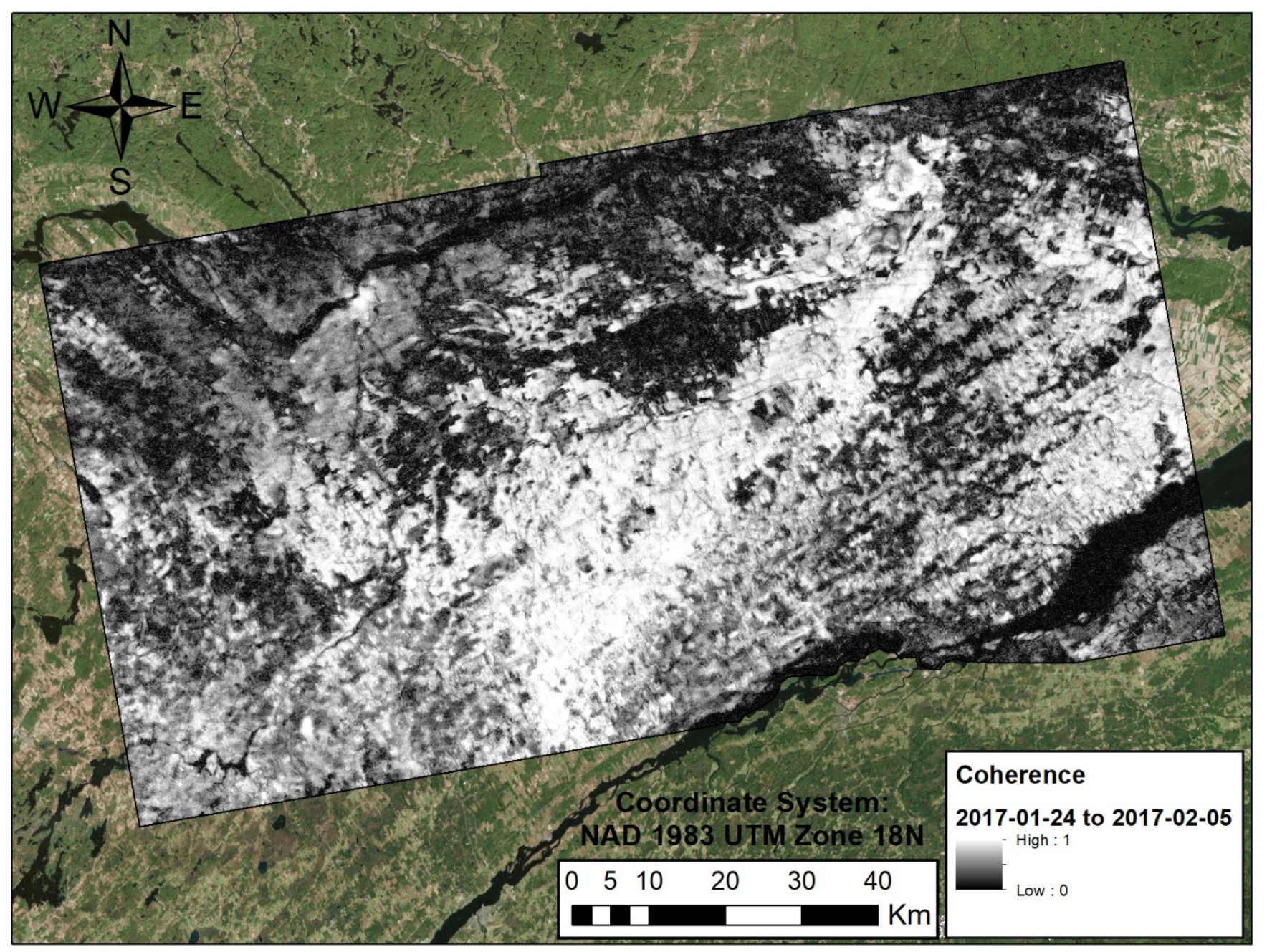

Figure 81: Interferometric coherence of the 2017-01-24 to 2017-02-05 Sentinel-1 interferogram. 


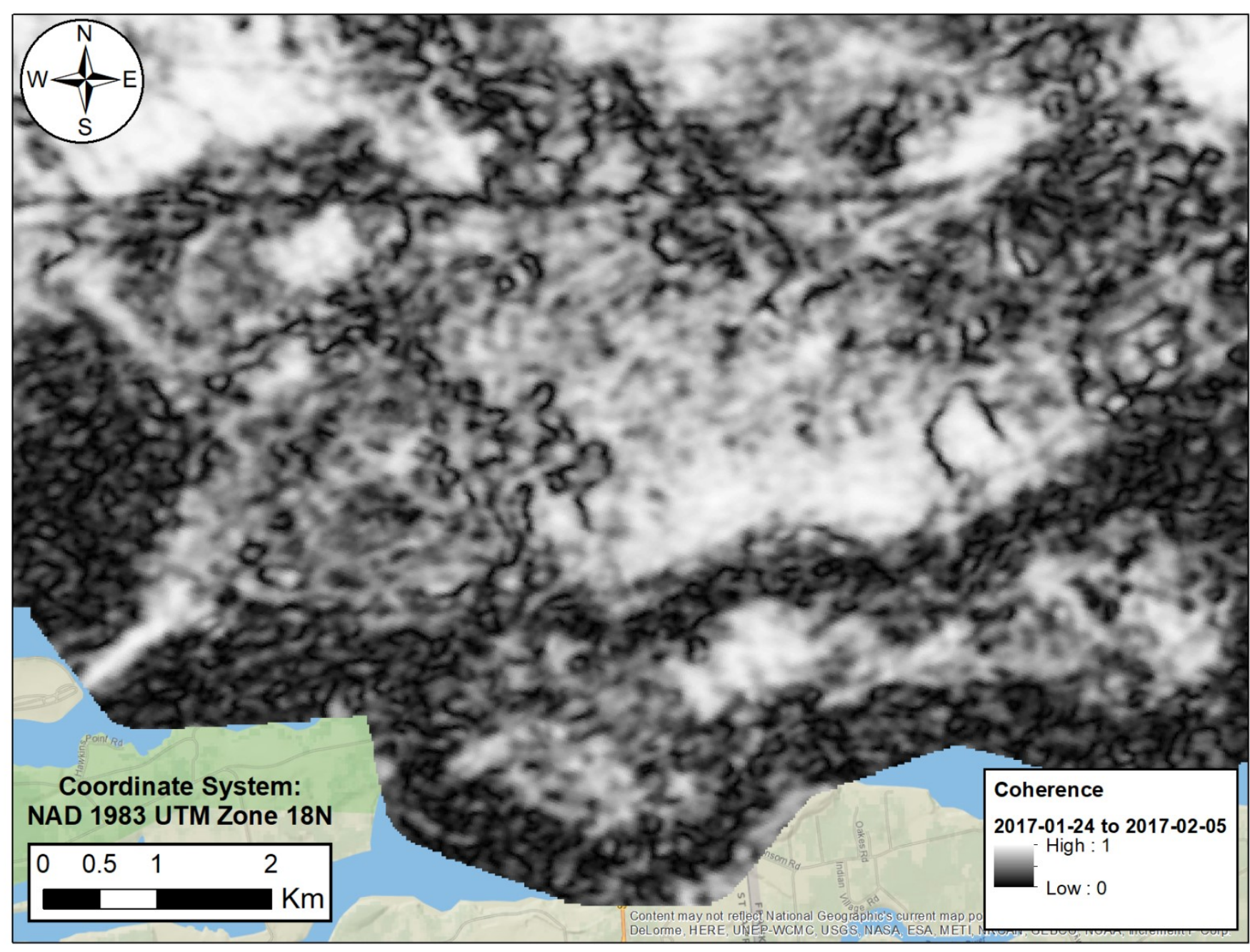

Figure 82: Interferometric coherence of Cornwall of the 2017-01-24 to 2017-02-05 Sentinel-1 interferogram. 


\section{Appendix E: Weather Radar at Sentinel-1 acquisition times}

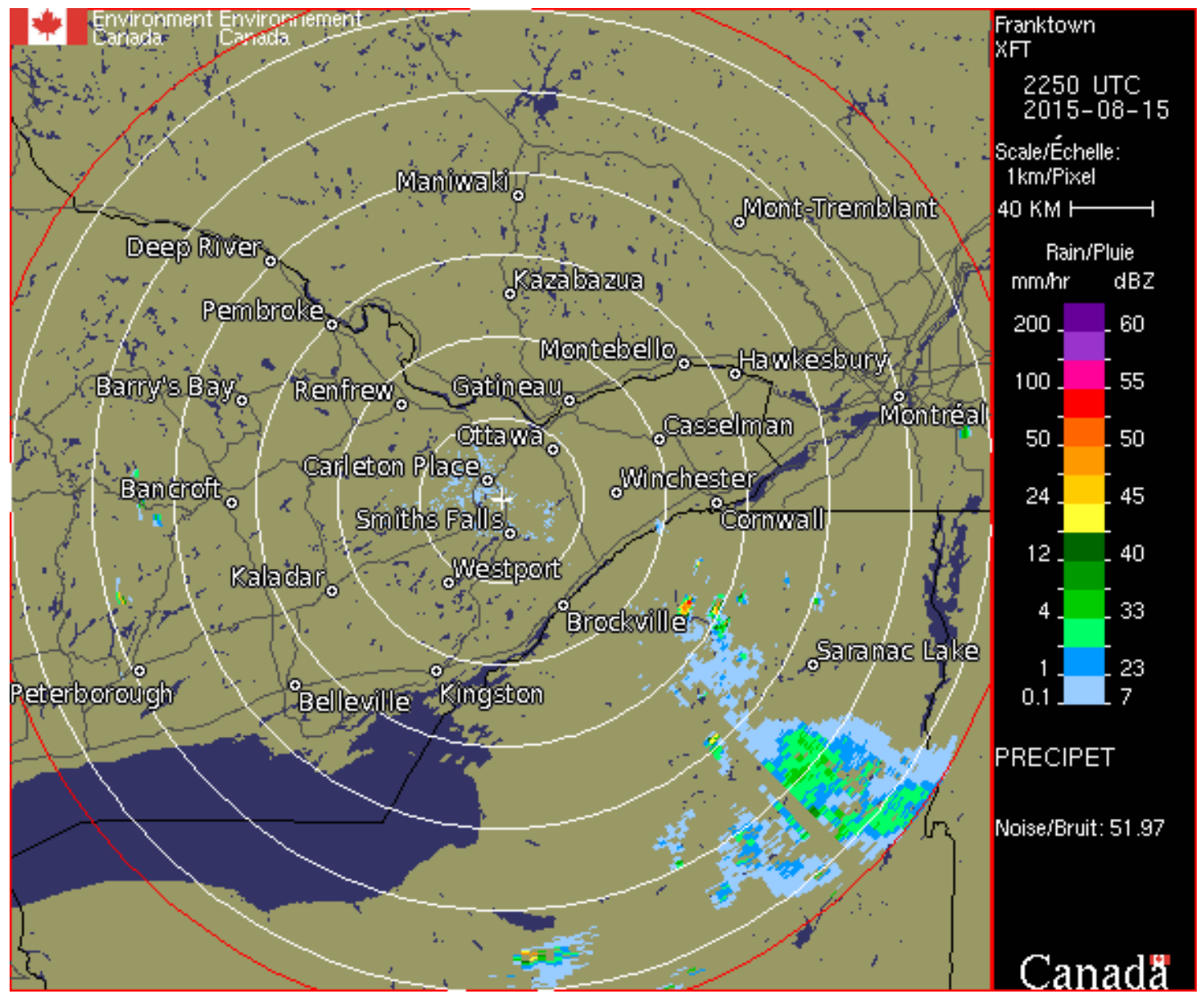

Figure 83: Weather radar image showing precipitation in the study area at 22:50 UTC on 2015-08-15. 


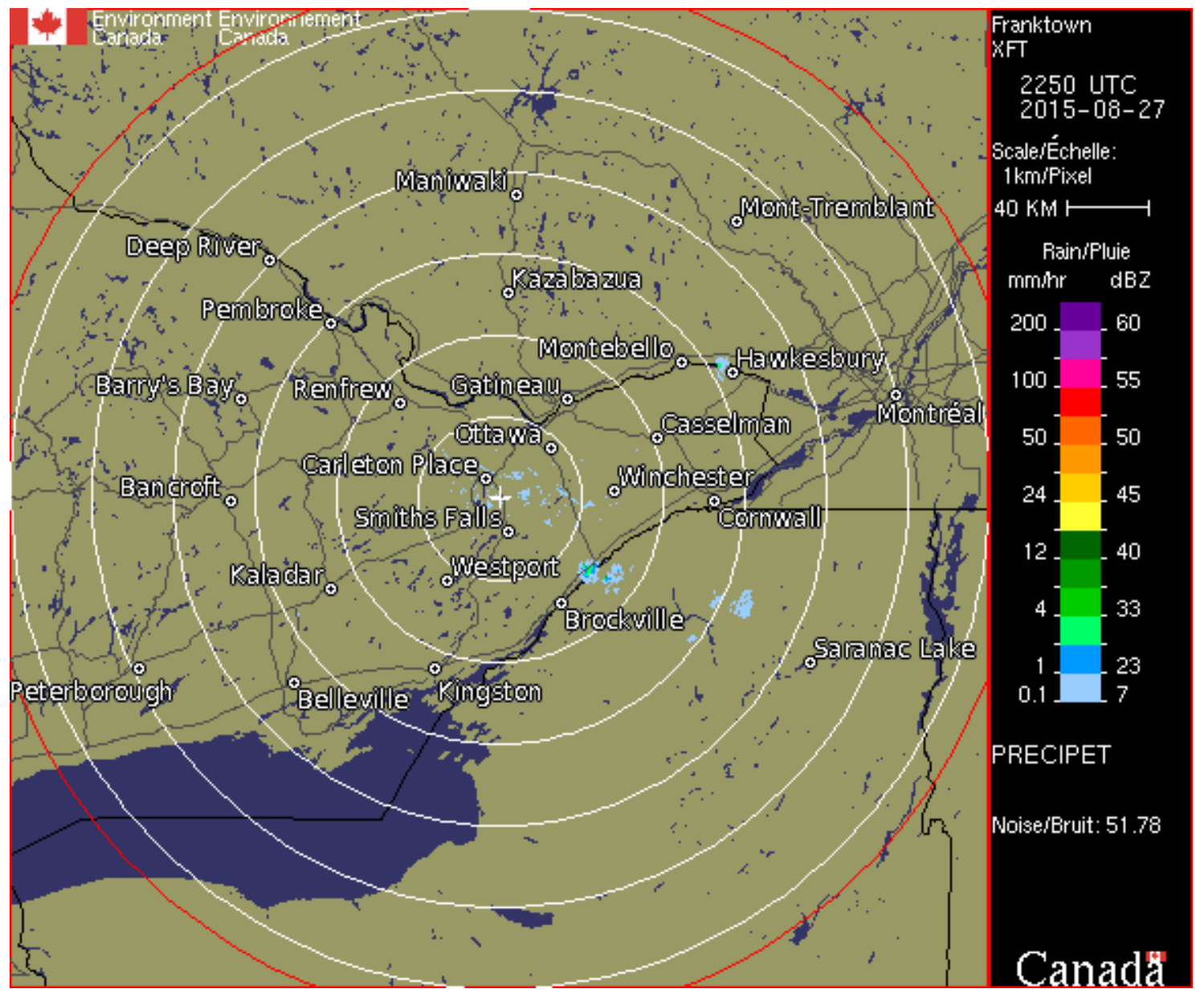

Figure 84: Weather radar image showing precipitation in the study area at 22:50 UTC on 2015-08-27. 


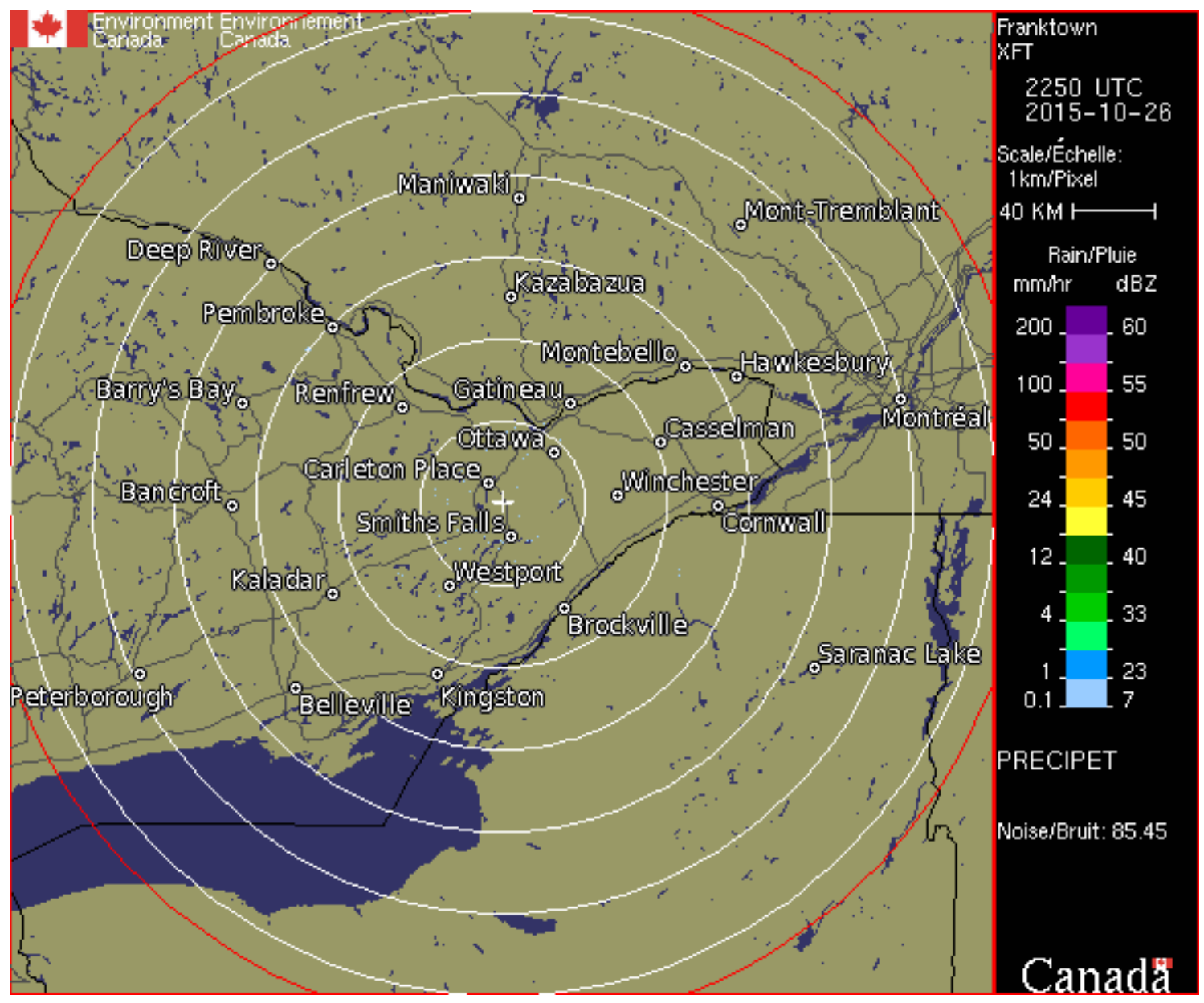

Figure 85: Weather radar image showing precipitation in the study area at 22:50 UTC on 2015-10-26. 


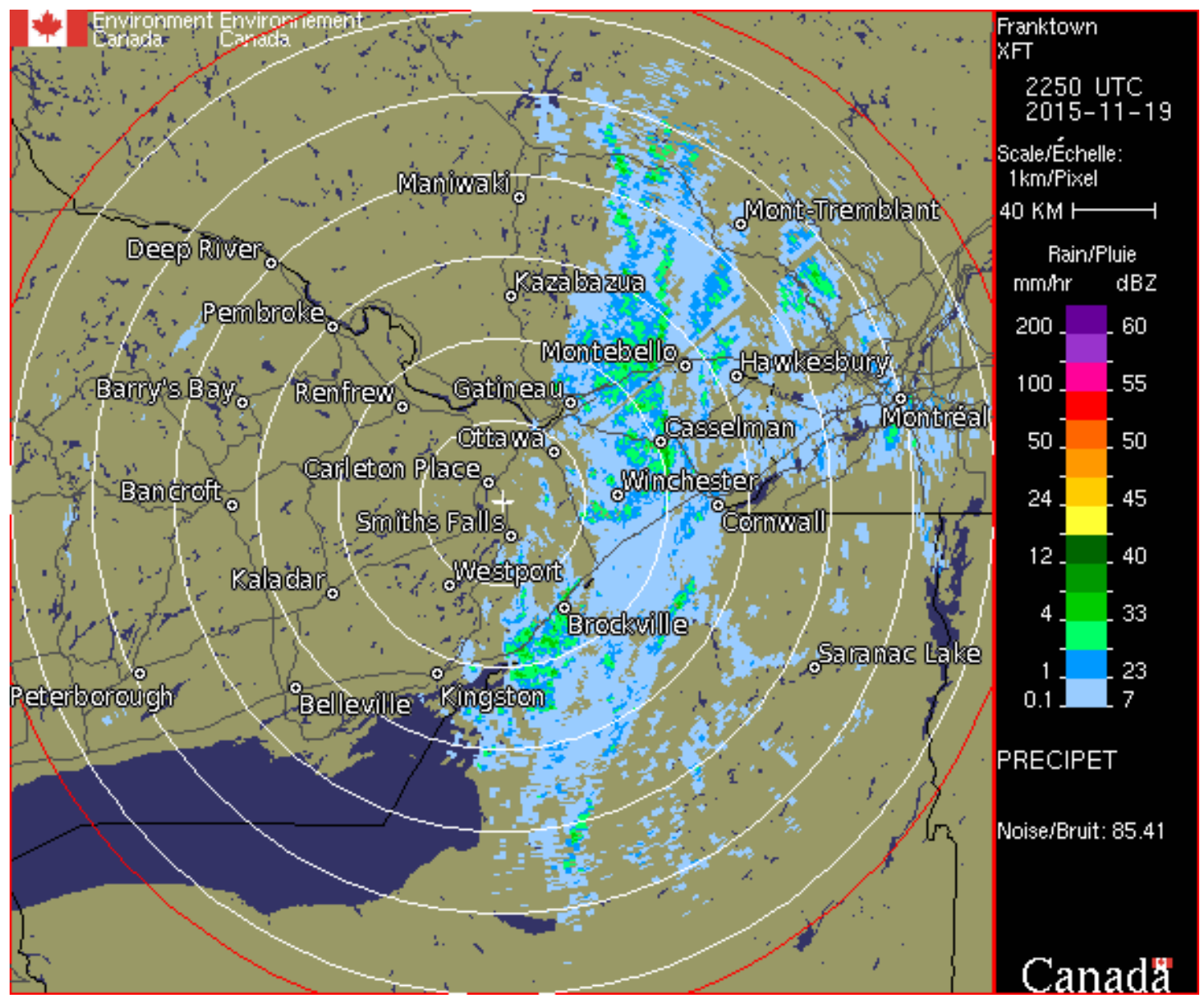

Figure 86: Weather radar image showing precipitation in the study area at 22:50 UTC on 2015-11-19. 


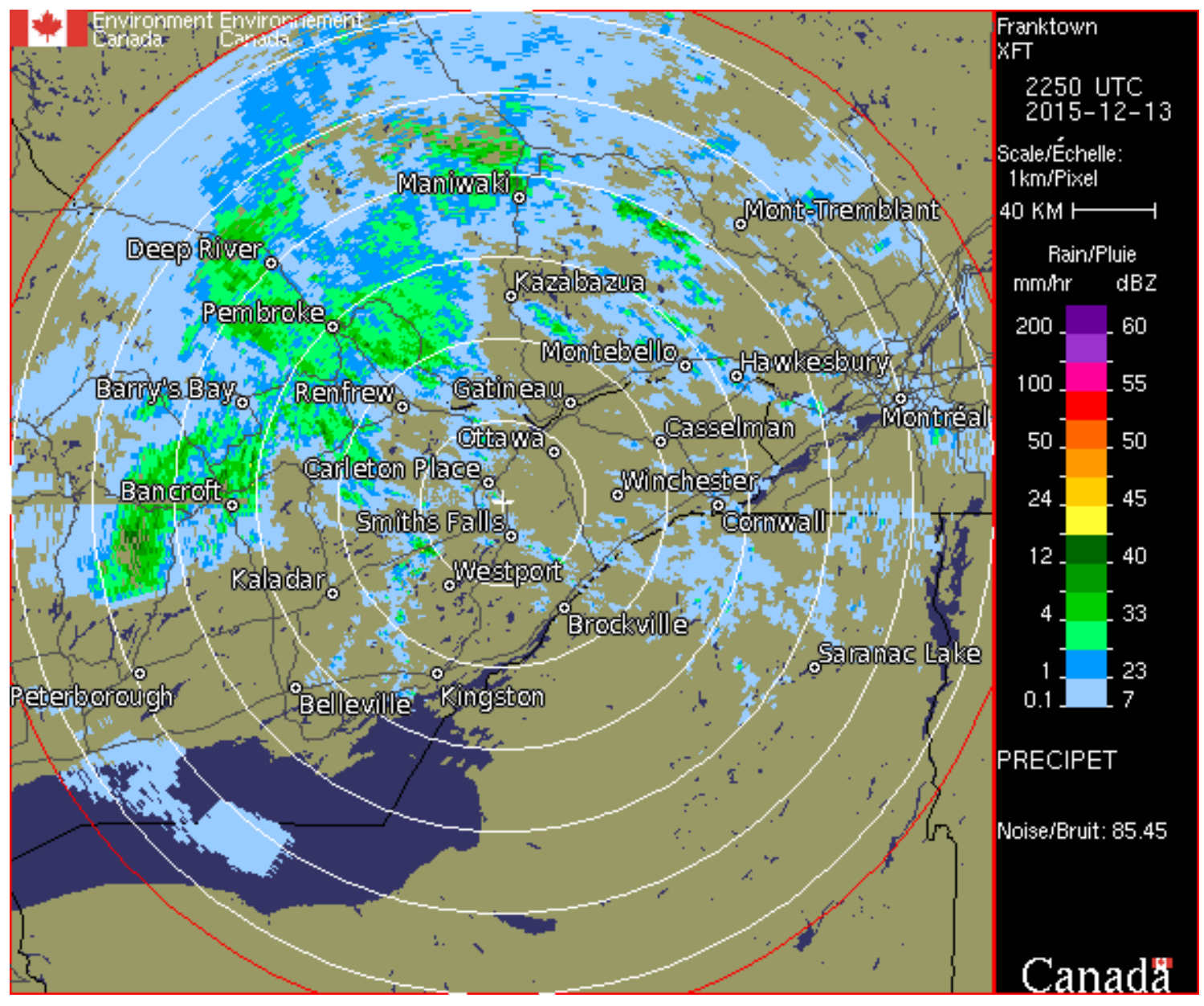

Figure 87: Weather radar image showing precipitation in the study area at 22:50 UTC on 2015-12-13. 


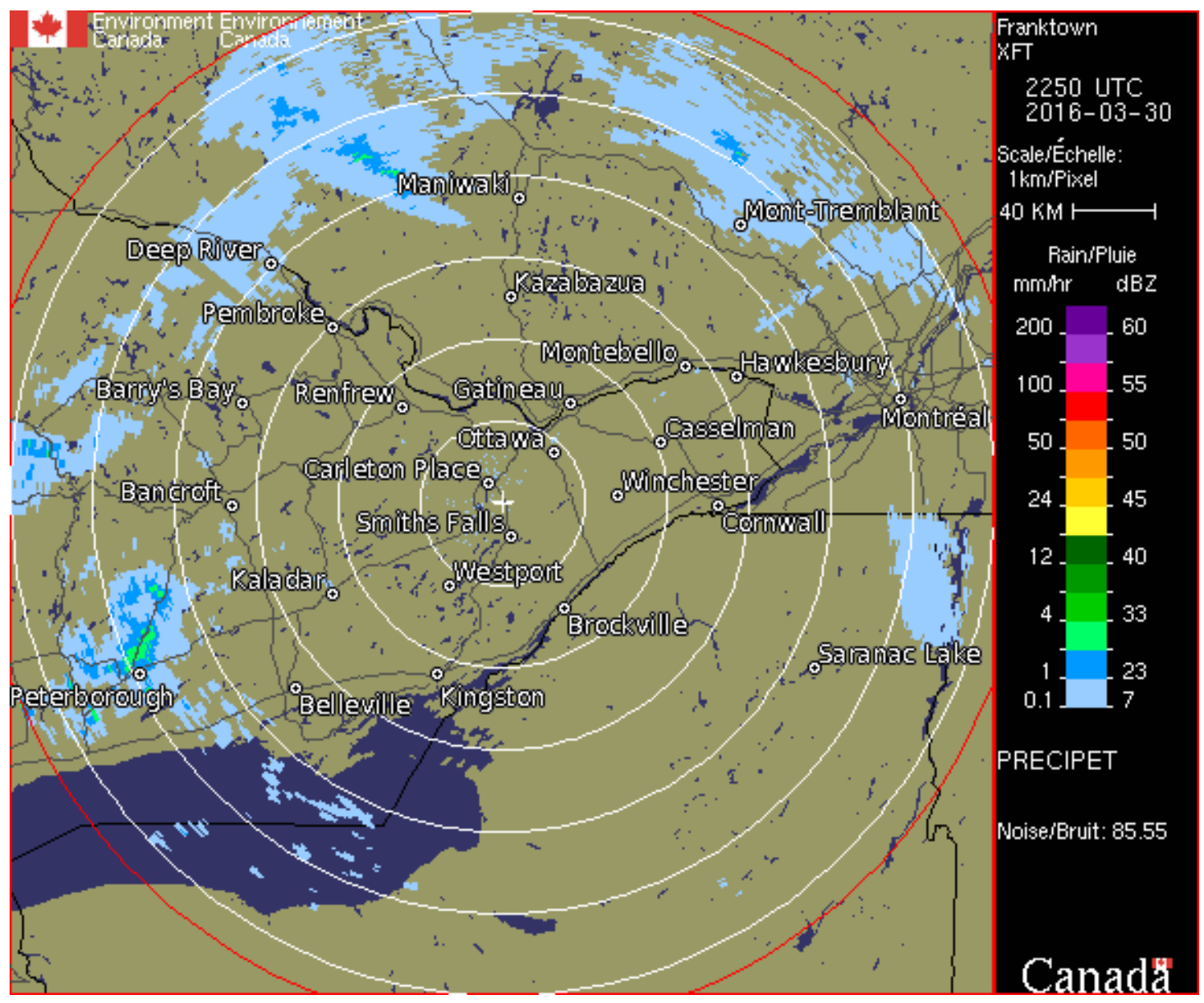

Figure 88: Weather radar image showing precipitation in the study area at 22:50 UTC on 2016-03-30. 


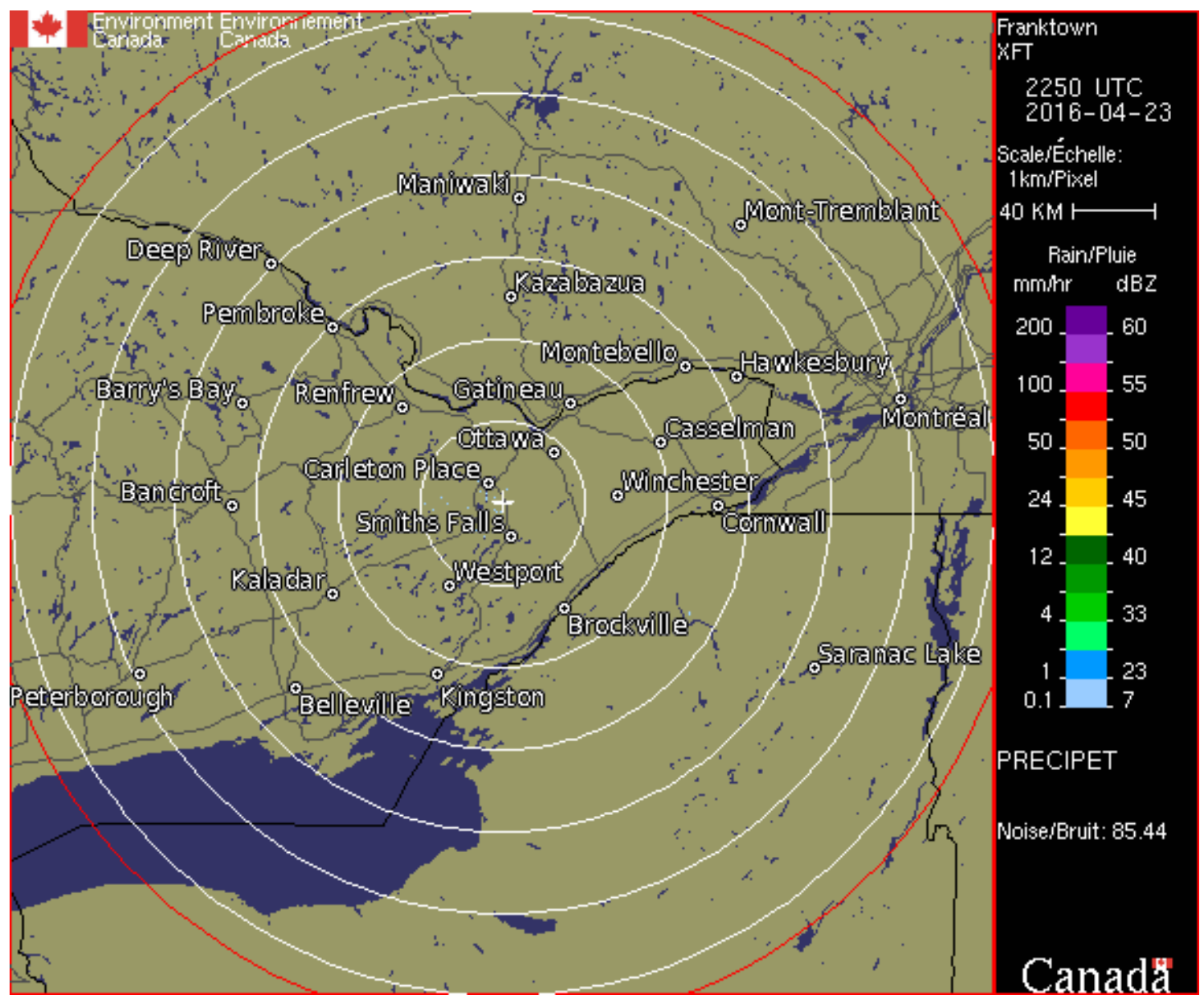

Figure 89: Weather radar image showing precipitation in the study area at 22:50 UTC on 2016-04-23. 


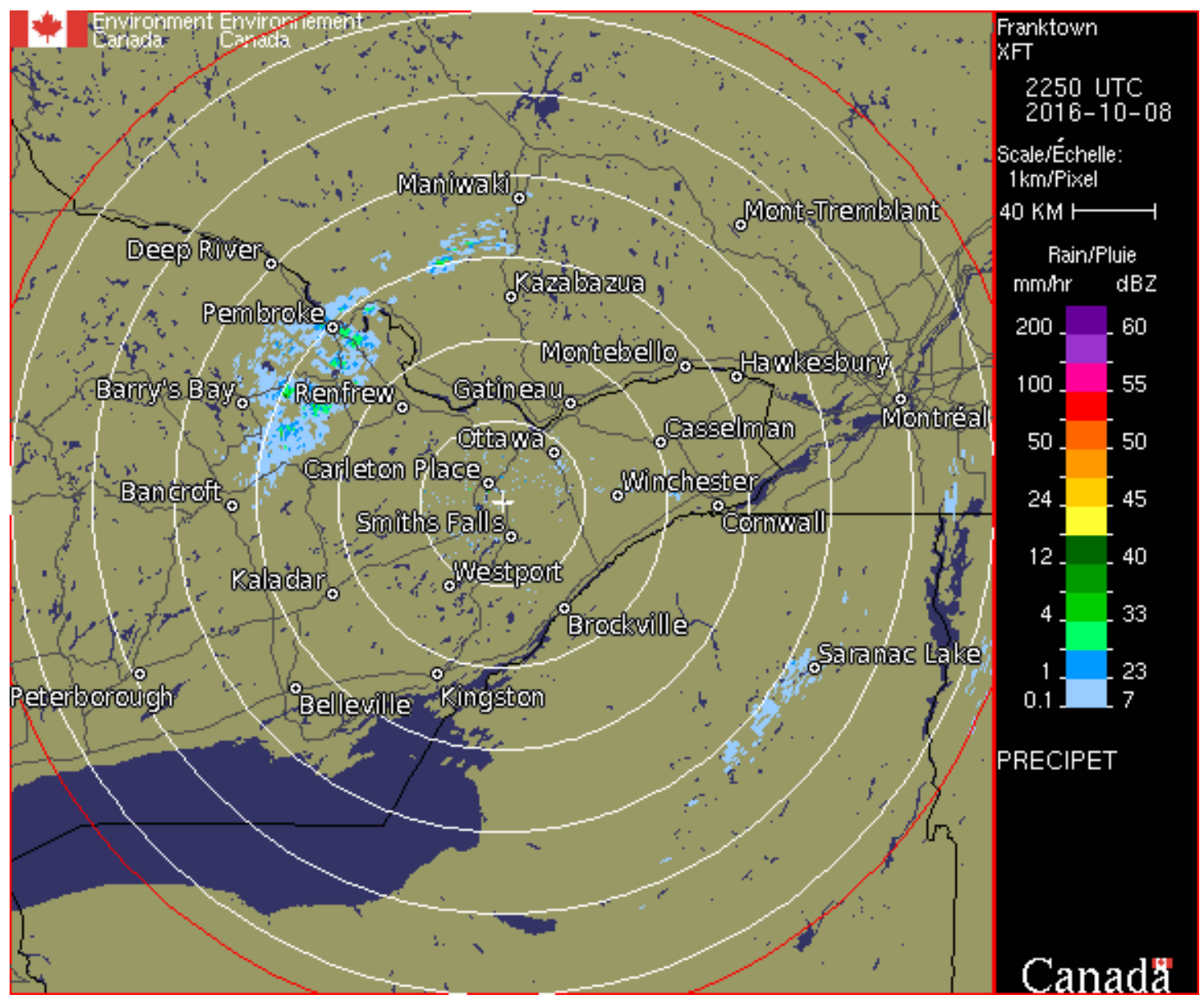

Figure 90: Weather radar image showing precipitation in the study area at 22:50 UTC on 2016-10-08. 


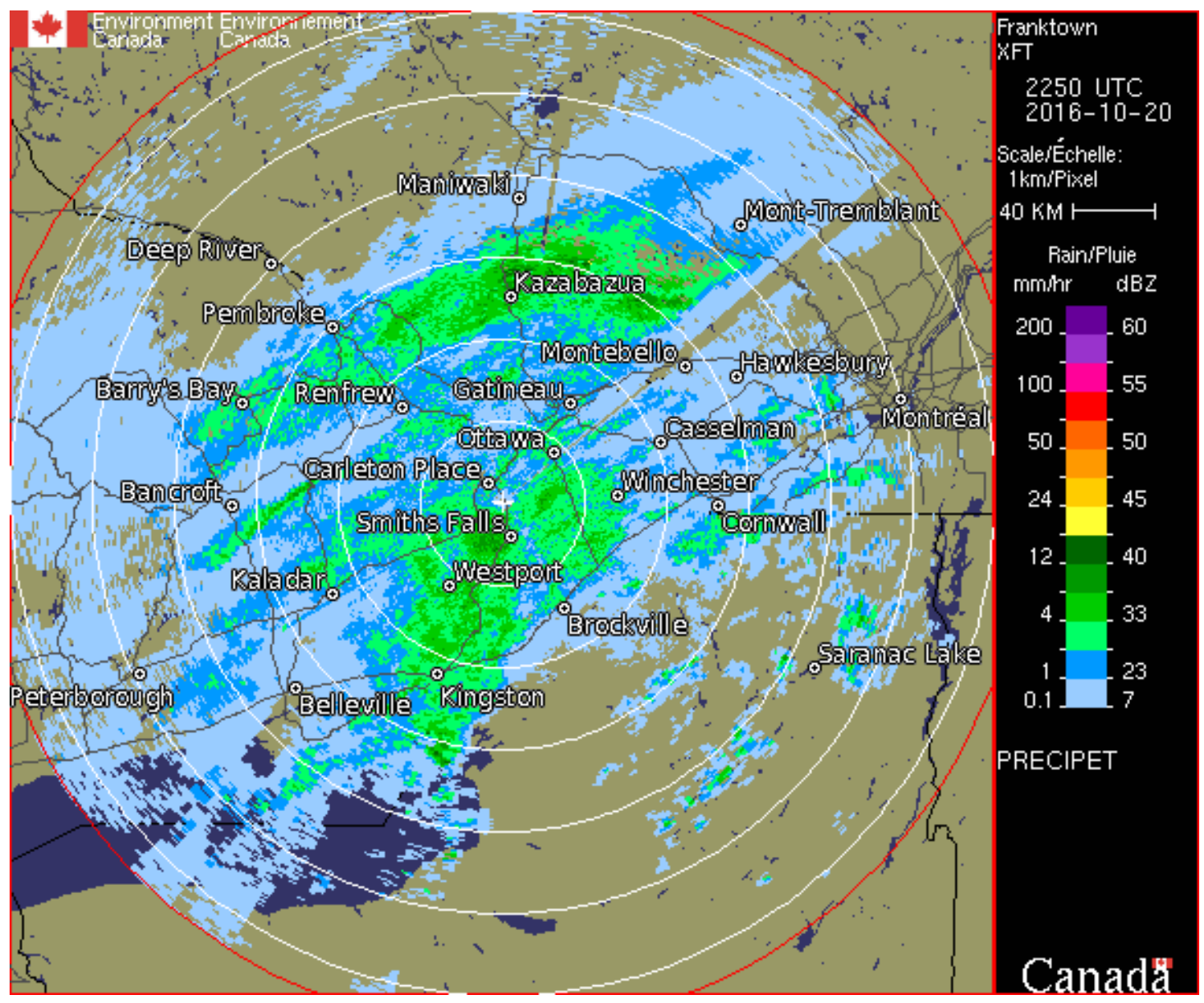

Figure 91: Weather radar image showing precipitation in the study area at 22:50 UTC on 2016-10-20. 


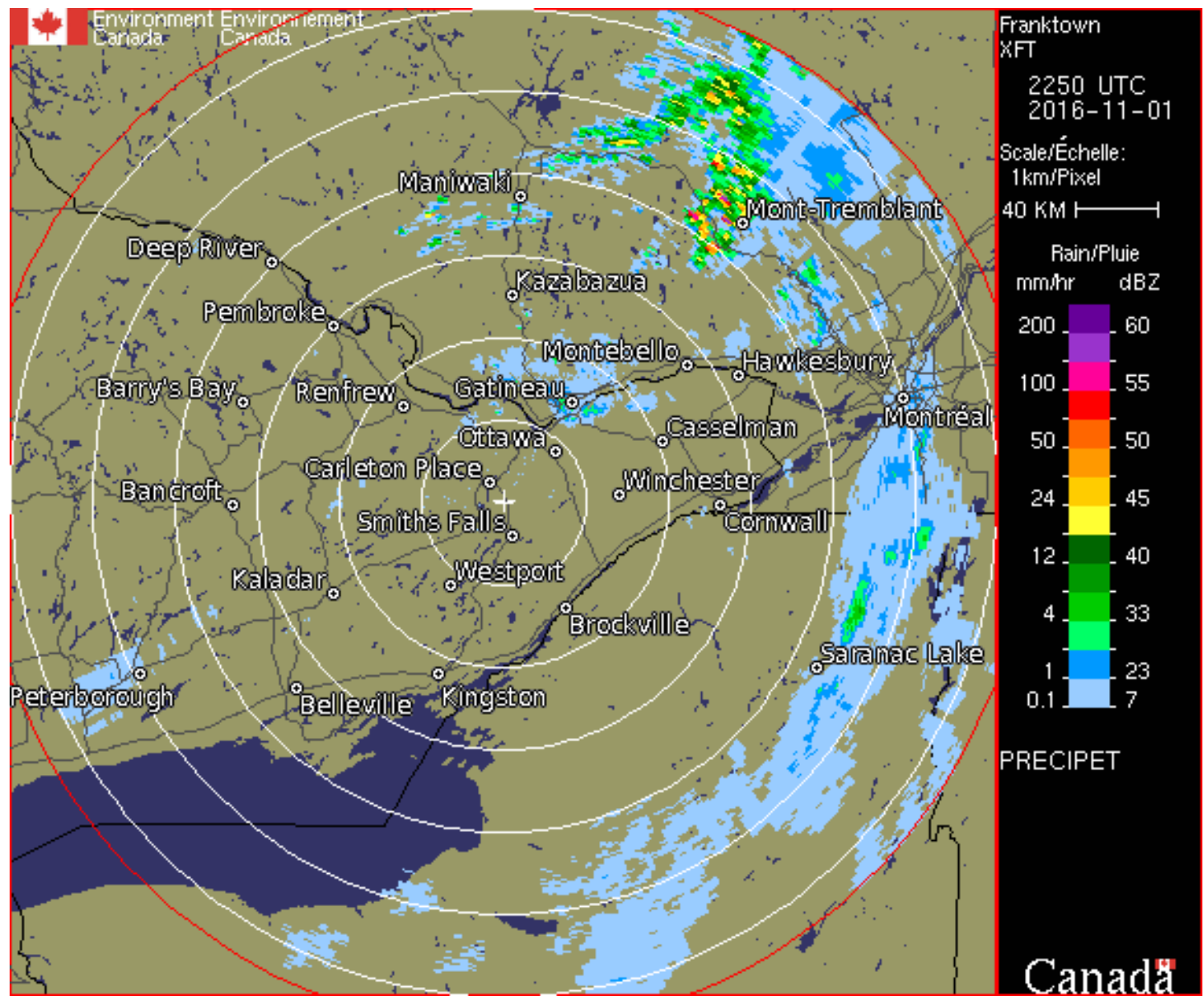

Figure 92: Weather radar image showing precipitation in the study area at 22:50 UTC on 2016-11-01. 


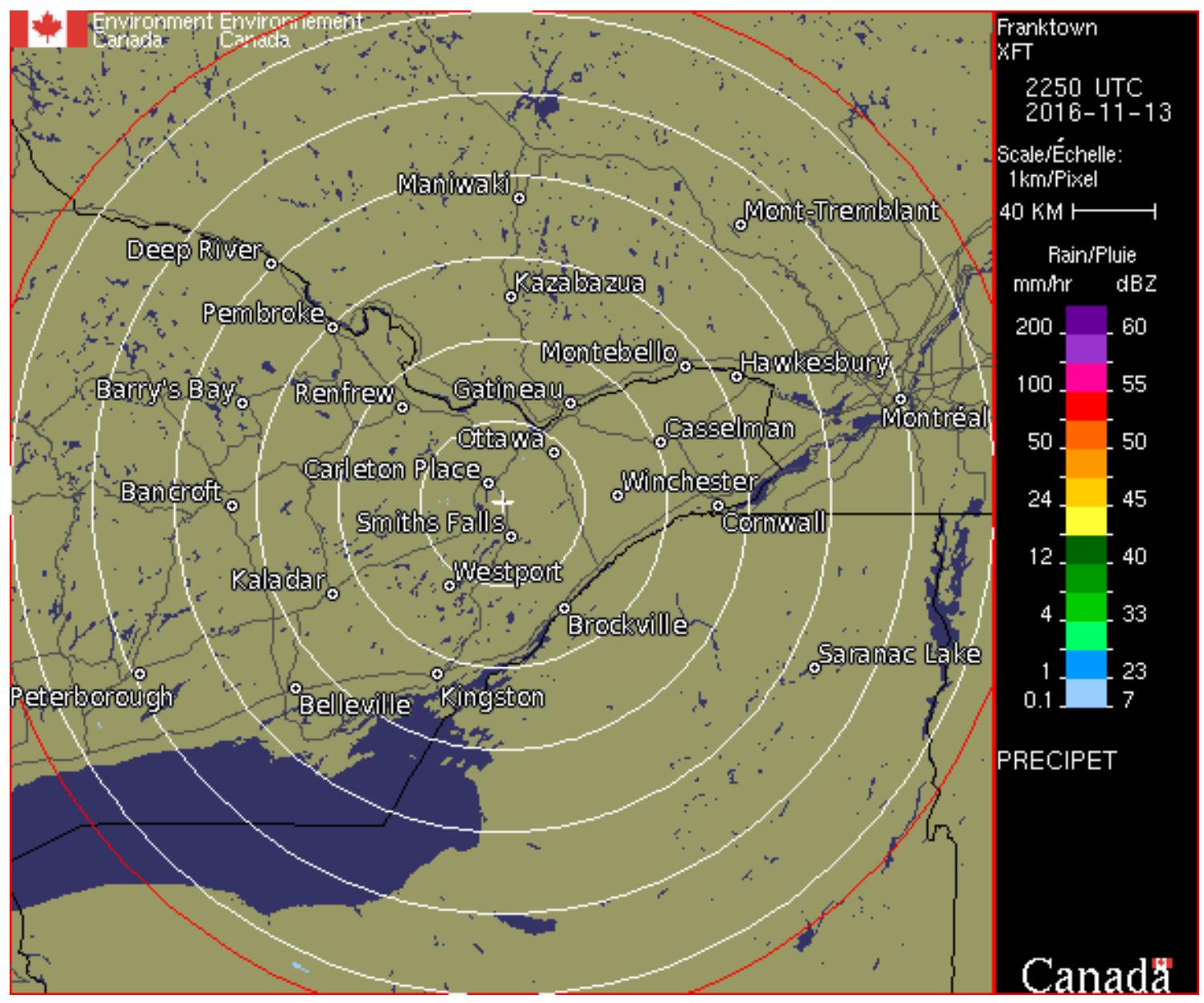

Figure 93: Weather radar image showing precipitation in the study area at 22:50 UTC on 2016-11-13. 


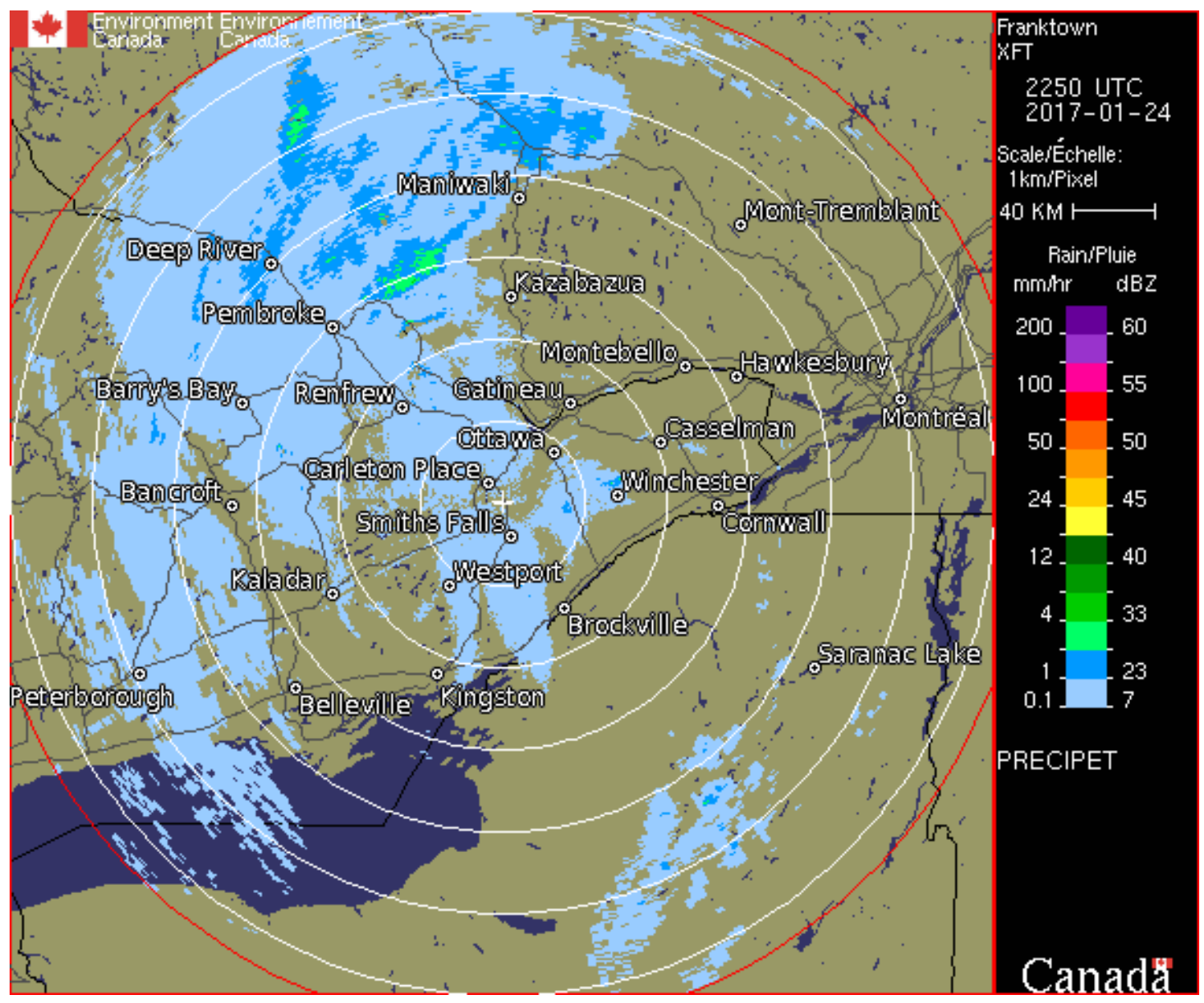

Figure 94: Weather radar image showing precipitation in the study area at 22:50 UTC on 2017-01-24. 


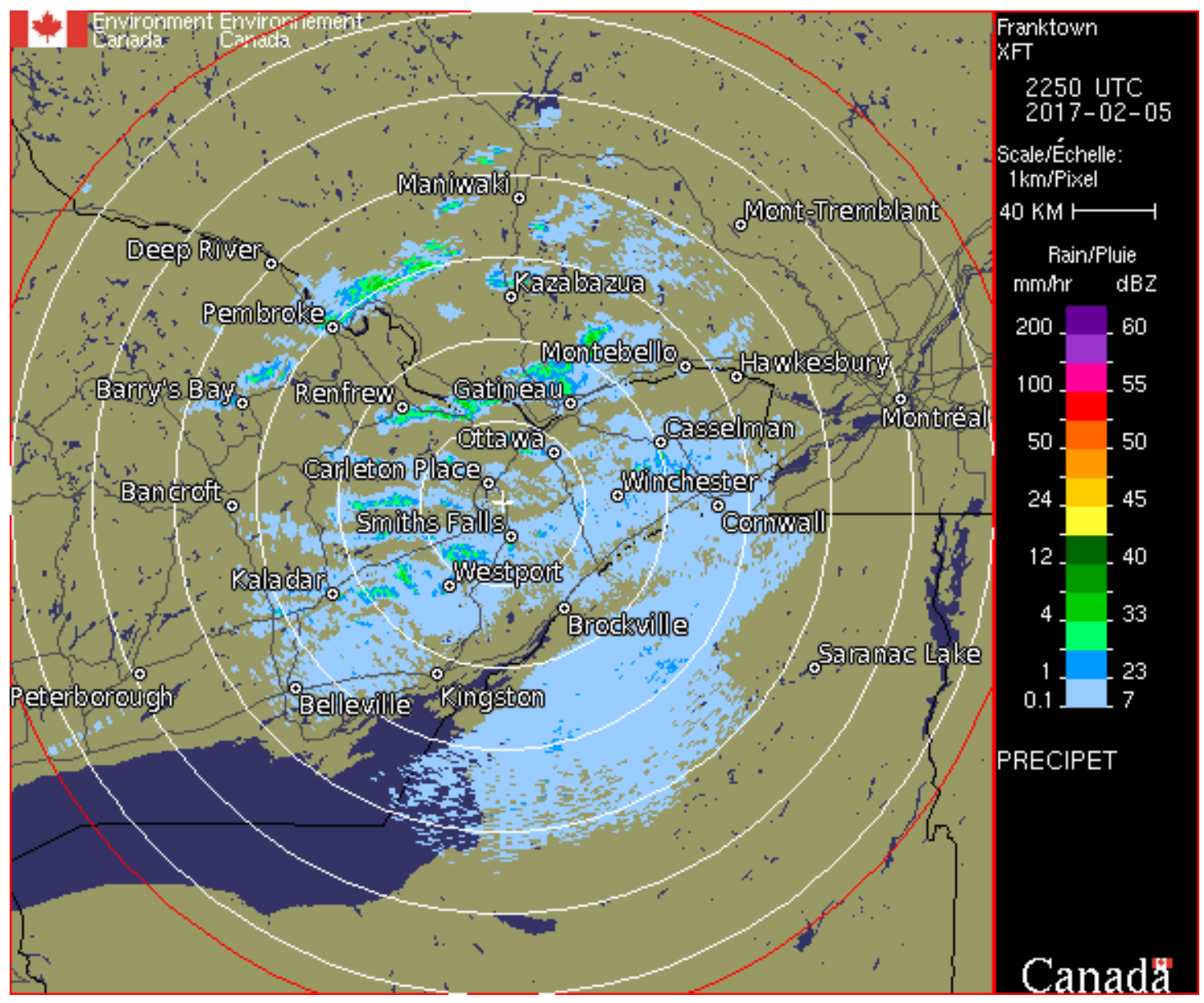

Figure 95: Weather radar image showing precipitation in the study area at 22:50 UTC on 2017-02-05. 